\title{
Sensitivity analysis methodologies for analysing emergence using agent-based models
}

\author{
Guus ten Broeke
}

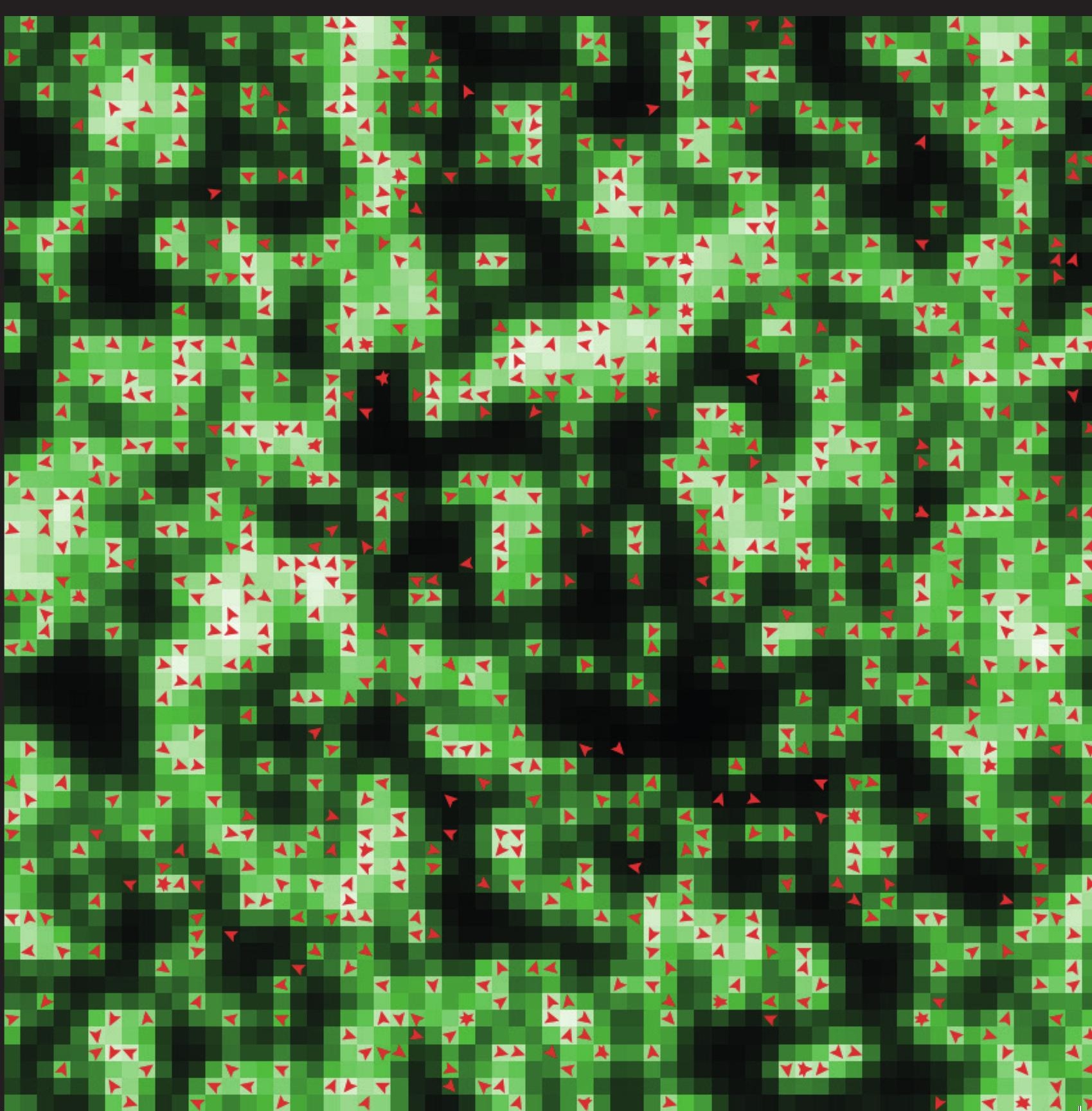




\section{Propositions}

1. Sensitivity analysis is a necessity for drawing general conclusions from simulation models.

(this thesis)

2. Global sensitivity indices yield little insight into emergent behaviour of agent-based models.

(this thesis)

3. Scientific publication of simulation models without complete documentation leads to a lack of replicability, and is therefore detrimental to scientific progress.

4. Metaphors about complex systems are usually more misleading than helpful.

5. To promote creative thinking, failure should be more appreciated than is presently the case in education and science.

6. The KISS (keep it simple, stupid) design principle (Axelrod, R. M. The complexity of cooperation: Agent-based models of competition and collaboration. Princeton University Press, 1997) applies as much to life as to modelling.

7. To fulfil a job optimally, it is best to spend your free time doing other things.

Propositions belonging to the thesis, entitled

'Sensitivity analysis methodologies for analysing emergence using agent-based models'

Guus ten Broeke

Wageningen, October 252017. 


\section{Sensitivity analysis methodologies for analysing emergence using agent-based models}

Guus ten Broeke 


\section{Thesis committee:}

\section{Promotor:}

Prof. Dr. J.Molenaar

Professor of Applied Mathematics

Wageningen University \& Research

\section{Co-promotors:}

Dr. G.A.K. van Voorn

Research Scientist, Mathematical and Statistical Methods Group

Wageningen University \& Research

Dr. Ir. A. Ligtenberg

Assistent Professor, Laboratory of Geo-Information and Remote Sensing

Wageningen University \& Research

\section{Other members:}

Prof. Dr. C. Kroeze, Wageningen University \& Research

Prof. Dr. C.K. Hemelrijk, Rijksuniversiteit Groningen

Dr.Ir. G.J. Hofstede, Wageningen University \& Research

Dr. B. Edmonds, Manchester Metropolitan University, UK

This research was conducted under the auspices of the C.T. de Wit Graduate School for Production Ecology and Resource Conservation (PE\&RC) 


\section{Sensitivity analysis methodologies for analysing emergent phenomena using agent-based models}

Guus ten Broeke

\section{Thesis}

submitted in fulfilment of the requirements for the degree of doctor at Wageningen University by the authority of the Rector Magnificus

Prof. Dr. A.P.J. Mol

in the presence of the

Thesis Committee appointed by the Academic Board to be defended in public on Wednesday 25 October 2017 at 4 p.m. in the Aula 
Guus ten Broeke

Sensitivity analysis methodologies for analysing emergence using agent-based models, 212 pages.

PhD thesis Wageningen University, Wageningen, NL (2017)

With references, with summaries in English and Dutch.

ISBN 978-94-6343-699-1

DOI http://dx.doi.org/10.18174/423172 


\section{Contents}

1 General Introduction 1

1.1 Complex adaptive systems . . . . . . . . . . . . . . . . 2

1.2 CAS models . . . . . . . . . . . . . . . . . . . 3

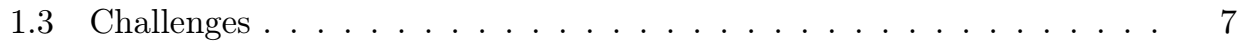

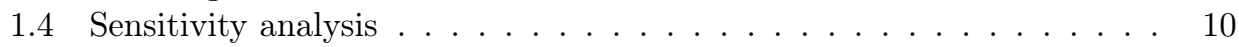

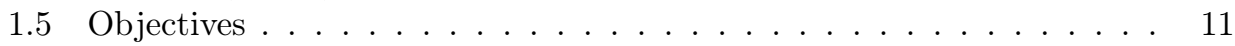

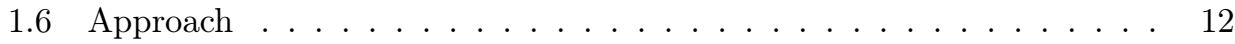

1.7 Thesis outline . . . . . . . . . . . . . . . . . . 12

2 Automated sensitivity analysis for ecological dynamical models 15

2.1 Introduction . . . . . . . . . . . . . . . . . 16

2.2 Local sensitivity analysis . . . . . . . . . . . . . . . . . . 18

2.3 Examples . . . . . . . . . . . . . . . . . . . 20

2.4 Discussion . . . . . . . . . . . . . . . . . . 26

2.A Local sensitivity analysis . . . . . . . . . . . . . . . . 27

2.B Ricker model . . . . . . . . . . . . . . . . . . . . . . . . 30

2.C Rosenzweig-MacArthur model . . . . . . . . . . . . . . . . 33

3 Detecting Tipping points with Sensitivity Analysis 43

3.1 Introduction . . . . . . . . . . . . . . . . . . 44

3.2 Sensitivity analysis methodologies $\ldots \ldots \ldots \ldots \ldots \ldots$

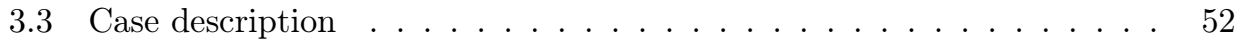

3.4 Results of sensitivity analysis . . . . . . . . . . . . . 55

3.5 Discussion \& Conclusions . . . . . . . . . . . . . . . . . . 63

3.A Direct differential method . . . . . . . . . . . . . . 65

3.B Local sensitivity analysis of the Bazykin-
Berezovskaya model

3.C Distribution of local sensitivity indices . . . . . . . . . . . . . 66

3.D Variance decomposition in Sobol' method . . . . . . . . . . . 67

3.E Sobol' indices in the steady state . . . . . . . . . . . . . . . . 69

3.F Sampling method for the Sobol' indices . . . . . . . . . . . 70

4 Which sensitivity analysis method should I use for my ABM? $\quad 75$

4.1 Introduction . . . . . . . . . . . . . . . 76

4.2 Selected goals for performing sensitivity analysis $\ldots \ldots \ldots \ldots . \ldots 77$

4.3 Selected methodologies for sensitivity analysis . . . . . . . . 78 
4.4 Model test case: agents harvesting a diffusing renewable resource . . . 81

4.5 Model results . . . . . . . . . . . . . . . . . . . . . . . . . 84

4.6 Discussion . . . . . . . . . . . . . . . . . . . . . . . . . . . . . . . . 92

4.A Model description . . . . . . . . . . . . . . . . . . . . . . . 96

4.B OFAT results . . . . . . . . . . . . . . . . . . . 104

4.C Consequences of the choice of sampling design . . . . . . . . . . . 104

5 Resilience through adaptation $\quad 111$

5.1 Introduction . . . . . . . . . . . . . . . . . . . . 112

5.2 Materials and Methods . . . . . . . . . . . . . . . . . . . 113

5.3 Results. . . . . . . . . . . . . . . . . . . . . 120

5.4 Conclusions \& Discussion . . . . . . . . . . . . . . . . . . . . . . 128

5.A Stationarity and ergodicity tests . . . . . . . . . . . . . . 131

5.B Computation of the earth mover's distance. . . . . . . . . . . . . . . . 133

5.C Results of stationarity and ergodicity tests. . . . . . . . . . . . . . 134

5.D Default parameter setting . . . . . . . . . . . . . . 139

6 Cooperation can improve resilience 141

6.1 Introduction . . . . . . . . . . . . . . . . . . . . . . . . 142

6.2 Model Description . . . . . . . . . . . . . . . . . . . . . . . . . . . . . . . . 144

6.3 Model analysis methodology . . . . . . . . . . . . . . . . . . . 146

6.4 Results . . . . . . . . . . . . . . . . . . . . 151

6.5 Conclusions \& Discussion . . . . . . . . . . . . . . . . . . . . . 159

6.A Model Description . . . . . . . . . . . . . . . . . . 161

6.B One-factor-at-a-time results for growth rate $r \ldots \ldots . . . . . . . .175$

6.C Global sensitivity analysis . . . . . . . . . . . . . . . . 175

7 General Discussion $\quad 179$

7.1 Aims of sensitivity analysis . . . . . . . . . . . . . . . . 179

7.2 Evaluation of sensitivity analysis methodologies . . . . . . . . . . . . 180

7.3 Lessons from applying sensitivity analysis to ABMs . . . . . . . . 183

7.4 Future work . . . . . . . . . . . . . . . . . . 185

$\begin{array}{lr}\text { Bibliography } & 189\end{array}$

$\begin{array}{ll}\text { Summary } & 199\end{array}$

$\begin{array}{ll}\text { Summary } & 201\end{array}$ 
Chapter 1

General Introduction 


\subsection{Complex adaptive systems}

Human and natural systems usually show complex behaviour that cannot be understood by studying in isolation the constituent components. Examples include economic markets (Tesfatsion 2003), ecosystems (Levin 1998), supply networks (Nair et al. 2009), and land-use systems (Parker et al. 2003). All these systems are composed of a large number of components, which are influenced both by mutual interactions and by interactions with the environment. The components are autonomous in the sense that they have their own objectives, and make their own decisions in order to achieve these objectives. The system-level behaviour emerges from interactions between these components. Ecosystems, for example, contain large numbers of autonomous and interacting organisms. These individual organisms are aimed at achieving individual-level goals, rather than system-level goals. At the system-level, these interactions form a balance that leads to more or less stable patterns over time, such as distributions of nutrient patterns and trace elements, or characteristic abundance distributions of species (Levin 2003). These patterns, which are important for sustaining life, emerge from mutual interactions between organisms, as well from interactions with their physical and chemical environment. The system as a whole appears to possess a great degree of coordination. Understanding this coordination is not possible by studying only the characteristics of the individual organisms or species, but requires studying the interactions within the system.

The framework of complex adaptive systems (CAS) is eminently appropriate for studying how complex behaviour at system level may emerge from interactions between lower-level components. A central idea behind CAS is that the mechanisms underlying this emergence share similarities across a wide range of systems. Thus, even though economies, ecosystems, and immune systems are entirely different systems, lessons from studying one of these types of systems, may be generalised to the other types.

There exists no universally accepted definition of CAS, but some essential properties are generally agreed upon (Railsback 2001, Holland 2002, Levin 2003).

- Autonomous agents. CAS are composed of autonomous units, or agents that act according to their individual objectives. Depending on the system, these agents may for example represent people, animals, or companies.

- Observation. Agents observe their environment, i.e., the state of their physical surroundings and the state of other agents.

- Observation-based actions. The observations of agents influence their decisionmaking process.

- Interactions. Agents have the capacity to make changes to their environment, and to influence other agents.

- Agent adaptation. As a result of interactions the characteristics of agent populations may change over time, for example through a process of natural selection.

- Learning. Agents can change their decision rules based on previous experiences, for example through trial-and-error, or imitation of other agents. Learning differs from adaptation, in that adaptation refers to changes in the distribution of 
characteristics across a population, whereas learning is based on the experience of individual agents.

- Emergence. The system-level properties of CAS cannot be a priori predicted from the properties of the agents, but are emergent.

Essential is that CAS is a bottom-up approach, in which system-level properties emerge from lower-level components. Some examples of this emergence are discussed in the following paragraphs, and it will remain a central concept throughout this thesis.

\subsection{CAS models}

Since the system-level behaviour of CAS emerges from lower-level interactions, it is typically difficult to analyse CAS using direct observations or experiments (Railsback and Grimm 2011). Whereas in physics, interactions between individual particles can be statistically aggregated to derive system-level properties, such an approach has proven to be of little use in the social sciences due to the complexity and diversity of interactions and heterogeneity of agents (Lansing 2003). For example, interactions in human societies are local, strongly non-linear, and dependent on individual differences between agents. Furthermore, keeping track of all the individual agents and interactions between agents in a system is typically not feasible in a practical situation (Gilbert 2004). Thus, understanding of the emergence of patterns in society has long remained out of reach.

Thanks to the development of computers, the field of social simulation has provided us with a tool for gaining insight into CAS. Simulation models are simplified representations of real-life systems in which interactions between agents can be modelled to investigate their effects at system level. The role of simulation models for studying CAS is comparable to that of thought experiments in physics (Holland 2002). They can be used to formalise assumptions about interactions between system components, and to systematically explore the consequences of these assumptions. In this way, simulation models allow us to perform 'experiments' that cannot be performed in real-life systems. Such experiments are of great help to develop theories on social processes and to generate new hypotheses.

It should of course be tested whether the assumptions behind any simulation model are appropriate, given the context of the study. Together with the conceptualisation and development of the simulation model, this testing is part of an iterative process. This process is commonly represented in terms of a modelling cycle (Fig 1.1). Based on the real-life system, a conceptual model is developed. Crucial to this development is the research question that the model is intended to answer, as well as the formulated hypotheses regarding this research question. The model should have the right level of detail to test these hypotheses (Grimm et al. 2005). A model is too simple if it does not take into account mechanisms that are essential to test the formulated hypotheses. If, in contrast, the model contains many unessential details, it may become impossible to analyse it in the context of these hypotheses. Once a conceptual model with the right level of detail has been developed, it is represented in terms of computer code. The model is then analysed to see what can be learned from it about the real world. Of 


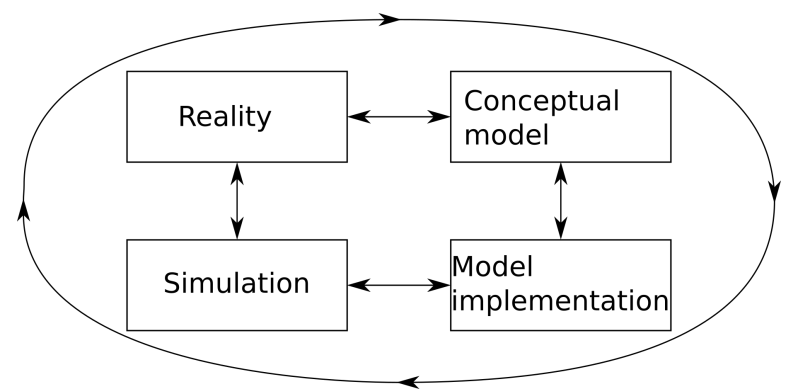

Figure 1.1: Modelling cycle (simplified from Refsgaard and Henriksen (2004), van Voorn et al. (2016)). Modelling often involves going back and forth between these steps, as denoted by the inner arrows. The cycle as a whole proceeds clockwise, as denoted by the arrows on the outer curve.

course, this cycle is a simplified representation of the modelling process. In practice, modelling often involves going back and forth between different steps, rather than going once through the cycle.

Various modelling methods have been used to describe CAS. In this thesis, we focus on two types of models, namely ordinary differential equation (ODE) models and agent-based models (ABM). These modelling methods are discussed below, including their strengths and weaknesses for modelling CAS.

\subsubsection{Ordinary differential equation models}

Differential equation models are used in a wide range of fields, such as physics, chemistry, engineering, biology, and economics. Fig 1.2a shows a graphical representation of differential equation models. A differential equation relates a function to its own derivative(s). In this thesis, only models with a single derivative with respect to time will be considered. Differential equations with a single independent variable (i.e. time) are referred to as ordinary differential equations (ODE)s. The vector field $d Y\left(t, \theta, Y_{0}\right) / d t=g\left(Y\left(t, \theta, Y_{0}\right), \theta\right)$ quantifies the change of the state variables $Y$ with respect to time, depending on the values of the parameters $\theta$ and initial conditions $Y_{0}$. Usually, $Y, Y_{0}$, and $\theta$ are vectors. Thus an ODE model may contain multiple state variables and parameters. The vector field of one state variable may depend on the values of other state variables. Such dependencies can be used to describe interactions between state variables. The model output $Y\left(t, \theta, Y_{0}\right)$ can be obtained by integrating the vector field over time. For some simple ODE models this integration can be done analytically, which then yields an explicit solution for the values of the state variables as function of time. For most models, no exact solution is available. Numerical methods may then be used to approximate the solution.

An example of an ODE model from ecology is the Lotka-Volterra model of predator-prey interaction. This example will be discussed here because ODE models of predator-prey interaction are extensively used in the second and third chapter of this thesis. The model reads as

$$
\frac{d Y_{1}}{d t}=\alpha Y_{1}-\beta Y_{1} Y_{2}
$$




$$
\frac{d Y_{2}}{d t}=\beta \delta Y_{1} Y_{2}-\gamma Y_{2}
$$

with initial conditions

$$
\begin{aligned}
& Y_{1}(0)=Y_{1,0} \\
& Y_{2}(0)=Y_{2,0} .
\end{aligned}
$$

Here $Y_{1}$ is the prey density, $Y_{2}$ the predator density, and $t$ time. The population densities $Y_{1}$ and $Y_{2}$ are functions of time, the initial conditions, and the model parameters so $Y_{1}=Y_{1}\left(t, Y_{1,0}, Y_{2,0}, \alpha, \beta, \gamma, \delta\right)$, and likewise for $Y_{2}$. The parameter $\alpha$ describes the intrinsic growth rate of the prey population, $\gamma$ the predator mortality rate, $\beta$ the predation rate coefficient, and $\delta$ the efficiency of biomass conversion. All symbols used in this chapter are listed in Table 1.1. Eqs $(1.1 \mathrm{a}, 1.1 \mathrm{~b})$ express the change in time of both populations as function of the population densities and model parameters. From these equations the population densities can be obtained as function of time, given values for the initial conditions $Y_{1,0}$ and $Y_{2,0}$.

Predator-prey ODE models like the Lotka-Volterra model are based on several assumptions that are important to consider in the context of CAS. Note that in Eq 1.1a the predator and prey populations, each of which consist of a large number of individual animals, are described by a single variable representing the population density. So, it is assumed that the population consists of identical individuals that do not adapt over time. Furthermore, the interactions between species are defined at the population level. It is thus assumed that each individual has an equal effect on each other individual (Huston et al. 1988). Although there are some possibilities for representing more detailed interactions or individual differences, ODE models are not suitable for representing local interactions between large numbers of heterogeneous agents. In many physical and social systems, however, it is important to consider locality of interactions and heterogeneity of agents. For example, in social systems people are influenced mostly by specific people in their surroundings, rather than being influenced equally by every member of the population. For systems in which local interactions between heterogeneous agents are expected to be important, ODE may not be the most suitable modelling method.

Even though ODE models may not be suitable for modelling local interactions and heterogeneous agents, such models are used extensively in the second of third chapters of this thesis. The reason is that some ODE models share important characteristics with CAS models, and the comparison with ODE models can yield valuable insights that are applicable to CAS models. One such property, central in chapters 3 and 4 of this thesis, is that the model may display various long-term behaviours depending on the initial conditions and parameter values. As an example, we again consider the Lotka-Volterra model 1.1a. It can be shown that for positive initial populations $\left(Y_{1,0}>0\right.$ and $\left.Y_{2,0}>0\right)$ the model converges to a long-term solution in which both populations oscillate periodically. Alternative solutions are found when $Y_{1,0}=0$ or $Y_{2,0}=0$. When $Y_{2,0}=0$, the system contains no predators, and the prey population will continue to grow exponentially. When $Y_{1,0}=0$, there is no prey, leading to extinction of the predator population. This example might seem trivial due to its simplicity, but in general ODE models can display various types of long-term behaviour and analysing this behaviour is usually no straightforward task. The tran- 
sitions between these types of behaviour in parameter space are referred to as tipping points.

An advantage of ODE models is the availability of well-developed tools for model analysis. Methods of bifurcation analysis (Kuznetsov 2004) are available to detect tipping points. Tipping points may also be an important property for other types of CAS models, as will be discussed in Section 1.3.1. But for these models bifurcation analysis is not applicable. In this thesis, methods to detect tipping points in CAS models will be proposed. ODE models will be used to test these methods by comparing their outcomes to those of existing bifurcation analysis methodologies.

Table 1.1: Symbols used in Chapter 1.

\begin{tabular}{lll}
\hline Symbol & Description & Units \\
\hline$Y$ & Model output & - \\
$Y_{1}$ & Prey population density & $\mathrm{kg} \mathrm{m}^{-2}$ \\
$Y_{2}$ & Predator population density & $\mathrm{kg} \mathrm{m}^{-2}$ \\
$Y_{1,0}$ & Initial prey population density & $\mathrm{kg} \mathrm{m}^{-2}$ \\
$Y_{2,0}$ & Initial predator population density & $\mathrm{kg} \mathrm{m}^{-2}$ \\
$t$ & Time & $\mathrm{S}$ \\
$\theta$ & Vector of model parameters & - \\
$\alpha$ & Prey growth rate & $\mathrm{s}^{-1}$ \\
$\beta$ & Ingestion rate & $\mathrm{s}^{-1}$ \\
$\gamma$ & Predator mortality rate & $\mathrm{s}^{-1}$ \\
$\delta$ & Yield over ingested biomass & - \\
$\phi$ & Potential & $\mathrm{J}$
\end{tabular}

\subsubsection{Agent-based models}

Agent-based modelling (ABM) is popular to study CAS. ABMs represent individual agents explicitly, and are thus ideally suited for including agent diversity and interactions between individual agents (Railsback and Grimm 2011, Gilbert 2008). As a result, ABM has become a preferred modelling tool across a range of fields, such as ecology, economics, and sociology (Macal 2016).

Definitions of what constitutes an agent strongly vary between authors (Macal 2016). Several necessary characteristics are considered here, summarised in Fig 1.2b. They directly correspond to some of the CAS characteristics listed in Section 1.1. Table 1.2 gives a comparison of CAS characteristics versus those of ABMs and ODE models. The most basic characteristic of ABMs is that an agent is an individual unit, with its own behavioural rules (Macal and North 2009). Secondly, an agent is autonomous (Macal 2016). This means that it has its own objectives, and can select behaviour based on its observations in order to fulfil these objectives. Thirdly, agents interact with other agents, and with their environment. These interactions may for example represent social interactions or exchange of goods or information. The CAS characteristics of agent adaptation and learning are not present in all ABMs, but are included in some. A useful categorisation of ABMs based on their characteristics, in particular adaptation and learning, has been given by Macal (2016). 


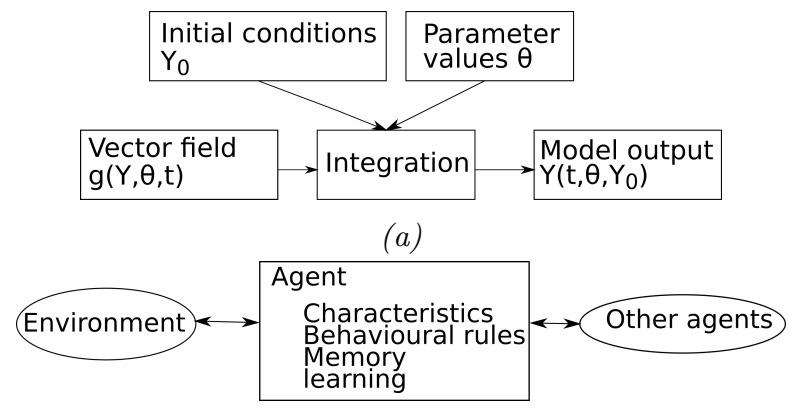

(b)

Figure 1.2: (a): A basic representation of an ODE model. To obtain the model output the vector field is integrated for given initial condition and parameter values. Note that the output variable $Y$, initial conditions $Y_{0}$, and parameters $\theta$ are vectors and can contain multiple values. (b): A basic representation of an ABM. The agents are autonomous entities that interact with the environment and with other agents. As model output we can choose any state variables of agents or the environment, the values of which emerge from the interactions in the model. Adapted from Macal and North (2009).

A demonstration of the advantages of ABMs over other modelling methods has been provided in a study on the immune system by Louzoun and Solomon (2004). In this study, the results from a differential equation model of an immune system were compared to those of an ABM in which the agents correspond to basic elements of the immune system. Since agent densities in the system are low and the interactions between agents are localised, it was found that the results of the differential equation model differ strongly from those of the ABM. Specifically, the ABM showed that the agents spontaneously formed 'macroscopic objects, which can move around, act in complex ways, and display adaptive behaviour' (Louzoun and Solomon 2004). The differential equation model, in contrast, predicted decay of the system.

\subsection{Challenges}

Although ABM provides a powerful tool for studying CAS, its utility is limited by difficulties in model analysis (Macal 2016, Crooks et al. 2008, Filatova et al. 2013). Most ABMs contain a number of parameters, which represent assumptions regarding various aspects of the modelled system. In practice, we may have some estimates for realistic parameter values, but most of the time these estimates are not very precise. Therefore, it is important to know how various parameters influence the model behaviour. This requires exploring the model behaviour under different parameter settings. Running a model only for some specific setting of parameter values yields information about its behaviour for only that setting. For other settings, however, the behaviour may be entirely different. However, running a model for all possible parameter combinations is usually not practically feasible.

If relationships between model parameters and output are not too complex, statistical tools may be used to gain understanding of model behaviour for various param- 
Table 1.2: Comparison between ODE models and ABM, based on utility for modelling various CAS properties. Since in ABMs agents are represented individually, ABMs are more suitable for modelling CAS characteristics like heterogeneous agents, agent adaptation, and learning.

\begin{tabular}{lll}
\hline CAS property & ODE & ABM \\
\hline Large numbers of agents & Represented as densities & Represented individually \\
Heterogeneity of agents & - & + \\
Interactions & At population-level & At individual-level \\
Observation-based actions & - & + \\
Agent adaptation & $\pm($ Geritz et al. (1997), & + \\
& Diekmann $(2003))$ & \\
Learning & - & +
\end{tabular}

eter settings, based on a limited number of model runs. For CAS models, however, it may be particularly difficult to study the relationships between the model's parameters and its behaviour. CAS models are aimed at investigating the emergence of different types of model behaviour from lower-level interactions. It is often not known in advance what kind of model behaviour should be expected. Furthermore, a number of specific CAS characteristics complicate the analysis of CAS models. In the following, a distinction will be made between characteristics related to complexity and those related to adaptivity, as summarised in Table 1.3. This distinction may seem simplifying, but will be shown to be useful because different types of characteristics complicate model analysis in different ways.

Complexity of a model refers to the number of agents, the number and type of agent behavioural rules, the number and non-linearity of interactions, the number of variables describing the physical environment, and rules for updating these variables. The high complexity of ABMs describing CAS ensures that these ABMs are likely to be strongly non-linear and involve many parameters and interactions. Furthermore, both the model parameters and the outputs may relate to different levels (i.e., the system-level, or the agent-level). Thus, there are multiple steps in between the model inputs and its outputs, and each of those steps can be strongly non-linear. Therefore, during model analysis it should not be assumed that the parameters affect the model output linearly, or that the output conforms to any specific statistical distribution, such as the normal distribution. Instead, methods are needed that are aimed at extracting as much information as possible on how each parameter affects the model behaviour, based on a limited number of model runs.

Adaptivity refers to changes in model behaviour over time. This includes agent adaptation, for example through natural selection, agent learning based on previous experiences, and the presence of feedback loops from the environment to the agents. Due to adaptivity, it is not sufficient to analyse what kind of state the model converges to, but it is necessary to consider how this state, and the model itself, change over time. 


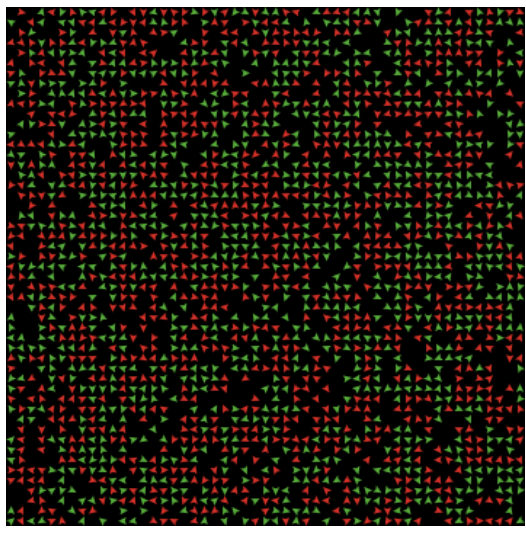

(a)

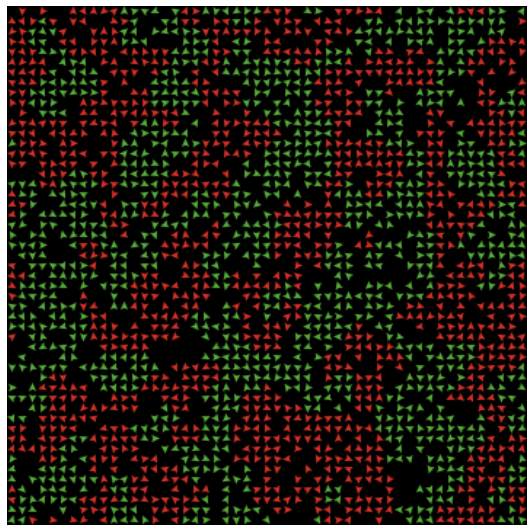

(b)

Figure 1.3: Snapshots of simulation runs of the Schelling model of social segregation. (a): For a small value of the tolerance parameter (0.05) the model converges to a mixed state in which there is no strong spatial clustering of the two colours. (b): For a larger value of the tolerance parameter the model converges to a segregated state in which both colours are clustered into separate neighbourhoods. The snapshots were made using the Netlogo implementation of the model (Wilensky 1997, 1999).

\subsubsection{Detection of tipping points and resilience}

ABMs may contain tipping points in parameter space where the model behaviour changes drastically. For example, predator-prey models like the one in Eq (1.1) often feature tipping points where one or both of the populations go extinct. An ABM example is the Schelling model of racial segregation (Schelling 1971). In this model agents of two colours live on a spatial grid, and may choose to change location based on the proportion of neighbouring agents of the same colour. A 'tolerance' parameter describes the minimum proportion of agents of the same colour each agent finds acceptable. Increasing the value of this parameter causes a transition from a nonsegregated state to a segregated state where agents of the same colour form spatial clusters (Fig 1.3). Whereas in the original model version this transition is gradual, in some model versions the state switches suddenly at a precisely determined critical parameter value (Stauffer and Solomon 2007). Sudden, qualitative switches in model behaviour, such as in the latter case, are typical for tipping points.

Since the Schelling model has only few parameters, the parameter value of the tipping point may be estimated by running the model for various parameter values. In contrast, many other ABMs have a large number of parameters, each of which may be relevant for detecting the tipping point. Since we have no explicit expressions that describe how the model output changes as a function of parameter values, we cannot detect tipping points using bifurcation analysis, as is possible for ODE models. Adaptivity further complicates tipping point detection in ABMs, because the model behaviour may continue to change over time rather than to converge to an unchanging steady state. Also, path dependence ensures that the model may evolve in different directions depending on initial conditions or stochastic effects. Currently, there are 
no reliable methodologies for detecting tipping points in ABMs.

A related challenge is the assessment of resilience. Resilience is a system property that is considered to be important for the management of social-ecological systems (SES), such as fisheries, land-use systems, and irrigation systems. Many SES are currently under pressure due to over-exploitation and changing conditions. Resilience refers to the capacity of a system to cope with such pressures or shocks while maintaining its identity and avoiding drastic consequences, such as as tipping points that push the system to an entirely different system state Walker et al. (2004), Scheffer (2009). Resilience is commonly illustrated in terms of potential landscapes, such as in Fig 1.4. The ball represents the current state of the system. For most CAS, this state is under constant influence of small disturbances, most of which do not have drastic consequences. Some disturbances, however, may cause the state to cross a tipping point and enter a different domain of attraction, leading to a qualitatively different system state. In a resilient system, the stability domain is wide and deep, so that only strong disturbances can cause the system to pass a tipping point. Adaptivity is considered to be an important component for the resilience of CAS, because due to adaptivity the shape of the stability landscape may change over time. Since tipping points in ABMs are difficult to detect, and the stability domains may change due to adaptivity, assessing the resilience of ABMs is challenging.

Table 1.3: Characteristics of complexity and adaptivity of CAS models.

\begin{tabular}{ll}
\hline Complexity & Adaptivity \\
\hline Number of agents & Adaptivity of agent characteristics \\
Heterogeneity of agents & Agent learning \\
Number of agent state variables & Strength of feedback loops \\
Number and type of agent & \\
behavioural rules & \\
Number of equations for & \\
environment & \\
Nonlinearity of equations for & \\
environment &
\end{tabular}

\subsection{Sensitivity analysis}

Sensitivity analysis is a commonly applied tool in the context of ABMs. It is aimed at examining how changes or uncertainties in the model parameters affect the model output. A number of standardised methods for sensitivity analysis exist (Saltelli et al. 2004a, 2008, Hamby 1994, Cariboni et al. 2007, Thiele et al. 2014). Most traditional methods focus on ranking the model parameters in terms of their influence on the model outcomes. Given assigned uncertainties to the input parameters, the resulting variation of the model output is quantified. In local methods of sensitivity analysis, only small parameter uncertainties around a reference point in parameter space are considered. By linearising the model output around the reference point, the sensitivity 


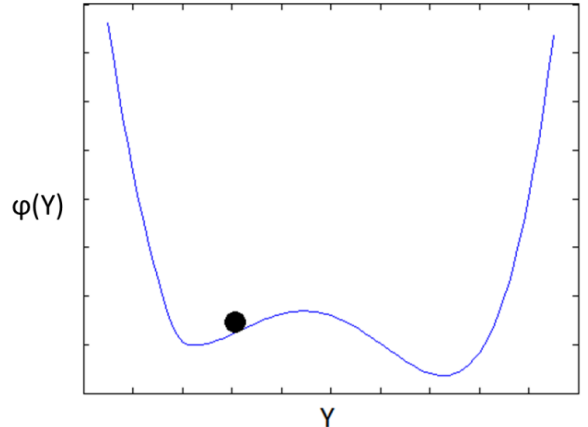

(a)

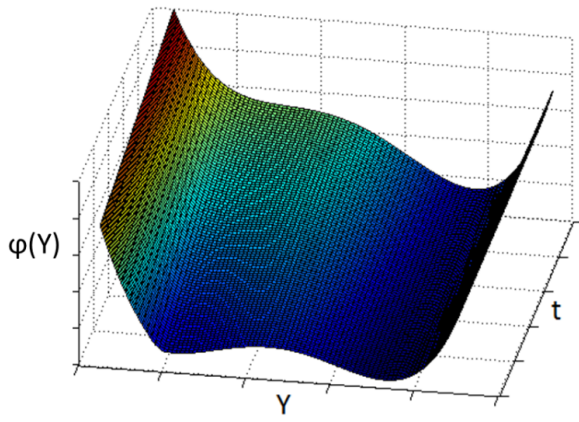

(b)

Figure 1.4: Hypothetical potential landscape $\phi(Y)$ as function of a state variable $Y$. The black ball represents the current system state, and the two valleys correspond to two separate stability domains. (a): In classical resilience studies, such as many engineering applications, the landscape is static. Resilience may be measured in terms of the width and height of the stability domain. (b): In CAS the stability landscape may change over time. In this example, the landscape is identical to (a) at $t=0$. Over time, the leftmost stability domain becomes less resilient and eventually disappears.

of a parameter may then be expressed in terms of the partial derivative of the model output with respect to that parameter. Global methods of sensitivity analysis consider large uncertainties across a wide range of parameter space. The variation of the model output across this region is typically quantified in terms of statistical measures, such as the variance. Sensitivities are expressed by decomposing this variation into terms attributed to (combinations of) parameters.

For CAS models the above approach may not yield insightful outcomes. Due to the complexity and adaptivity of these models, the model output and its sensitivities to the various parameter vary strongly across the parameter space and across time. By aggregating model output across parameter space, crucial information on the model behaviour may therefore be lost, as will be shown in Chapters 2 and 3 of this thesis. Specifically, the aggregated output will be of little value for detecting tipping points, or evaluating resilience. Furthermore, ABMs often produce results that could not easily have been predicted in advance. We cannot know in advance which output measures we should focus on to find these properties. Focussing only on a single output measure, and aggregating that measure across parameter space, would likely cause us to miss results that might be of interest (Nelson 2016). Thus, existing methods of sensitivity analysis are not sufficient for analysing ABMs and new methods are needed.

\subsection{Objectives}

Although traditional sensitivity analysis methods are not well-suited for ABM analysis, the topic of sensitivity analysis for ABMs has received relatively little attention and has therefore remained a stumbling block for applying ABM in practice (Macal 
2016, Crooks et al. 2008, Filatova et al. 2013). So, there is a need for new sensitivity analysis methods that are capable of dealing with the complexity of ABMs. The main aim of this thesis is to develop such methods. Specific issues that we will focus on are,

- The detection of tipping points in $\mathrm{ABM}$. Detecting tipping points is an important part of ABM analysis. In this thesis, we will examine the possibilities of using sensitivity analysis for this purpose. To validate our work, we will also apply sensitivity analysis methods to ODE models, where the outcomes can be compared directly to those of bifurcation analysis.

- Analysing adaptivity in ABMs. Adaptivity is an essential component of CAS. The sensitivity analysis methods to be developed in this thesis should allow to analyse the effects of adaptivity in ABMs.

- Analysing resilience of $\mathrm{ABM}$. Resilience is considered to be an important property of CAS. Adaptive agents may adjust their behaviour in response to pressures, and as such increase the resilience of the system in response to these pressures. The methods of sensitivity analysis in this thesis should be able to assess the resilience of the system.

\subsection{Approach}

Many ABMs are developed for specific case studies. In most practices, sensitivity analysis is performed after the model has been developed. It is then often impossible to fully analyse the model. This thesis will instead take a stepwise approach (Fig 1.5). A simple yet illustrative test-case ABM will be developed. The utility of various sensitivity analysis methodologies will be assessed by applying them to this test-case. Starting off with a simple test-case will help to pinpoint challenges in analysing ABMs, and to deal with these challenges in isolation. Once this test-case has been properly analysed, its complexity and adaptivity will be increased following the different paths shown in Fig 1.5. After each increase in complexity or adaptivity it will be assessed what kind of methods are needed to analyse the new model version. If available methods are found to be insufficient, new methods will be developed. In this way, the extensions of the test-case will guide the development of analysis methods that are suitable for dealing with specific CAS properties of ABMs.

In addition to the ABM test-case, we will also use ODE models as testing case for methods to detect tipping points. Since for ODE models methodologies of bifurcation analysis are available to detect tipping points, the results from newly developed methods can be compared to analytical bifurcation analysis results before applying them to ABMs.

\subsection{Thesis outline}

The thesis has the following structure. Chapter 2 will serve as an introduction to classical methods of local sensitivity analysis. The application of these methods is 


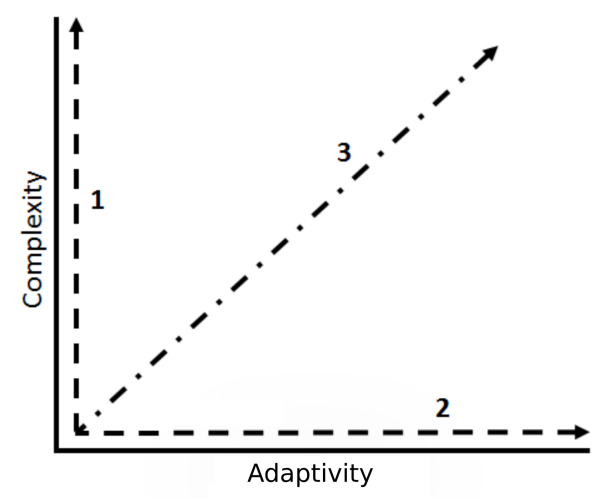

Figure 1.5: The approach in this thesis starts with the analysis of a 'minimal' CAS. Then, different paths will be followed, increasing (1) its complexity, (2) its adaptivity (2), and (3) both its adaptivity and its complexity.

demonstrated by using them to identify the most influential parameters of an ODE model for predator-prey interaction.

Chapter 3 describes the analysis of an ODE predator-prey model that contains several tipping points. At these tipping points, the asymptotic behaviour changes between stable coexistence, limit cycles, and extinction. It is shown how the presence of tipping points complicates the interpretation of sensitivity analysis results, and the use of sensitivity analysis methods for tipping point detection is explored. The sensitivity analysis results are verified through comparison with analytical results from bifurcation analysis.

In Chapter 4, an $\mathrm{ABM}$ is introduced in which agents compete for a commonpool resource. This ABM serves as a test-case for sensitivity analysis, and although it is simple, it still possesses most of the characteristics that complicate sensitivity analysis of ABMs. Adaptivity is still left out. The model is analysed using three standard methodologies of sensitivity analysis, and the strengths and weaknesses of these methods for analysing ABMs are evaluated.

In Chapter 5, the ABM that was introduced in Chapter 4 is expanded by the inclusion of a mechanism for agent adaptation through a process of natural selection. A sensitivity analysis method to quantify the effects of adaptation over time is developed. Furthermore, it is shown how adaptation affects the resilience of the system against pressures.

In Chapter 6 an extension of the ABM discussed in Chapters 4 and 5 is analysed. In this extension direct interactions are added in the form of cooperation between agents. Furthermore, a learning process for individual agents is included for making decisions regarding cooperation. It is investigated how various mechanisms affect the level of cooperation within the system. Furthermore, it is investigated how cooperation affects the resilience of the system.

Chapter 7 concludes with a general discussion. The achievements of this thesis are summarised and discussed. Furthermore, opportunities for further progress are indicated. 



\section{Chapter 2}

\section{Automated sensitivity analysis for ecological dynamical models}

G.A. ten Broeke, E.H. van Nes, L. Hemerik, G.A.K. van Voorn.

To be submitted. 
Ecological systems are often studied using dynamical models. These models have to be properly validated in order to be useful. Validation entails the use of data, which is usually available only for a limited number of variables and short ecological time scales. Such analysis therefore has a strong focus on transient model behaviour. Sensitivity analysis can therefore be a key tool to help this type of model analysis: it quantifies the effects of changes in model parameters and inputs on the model predictions, and thus allows for pinpointing influential parameters and inputs as possible targets for validation experiments. Performing a sensitivity analysis can, however, be costly and cumbersome. In this paper we present a tool in MATLAB for automated sensitivity analysis as an aid for ecological modellers and experimentalists. We demonstrate the tool's performance by comparing its results with semi-analytical results for two examples. Our study shows that the user-friendly tool quickly yields accurate sensitivities that help in model parametrisation and analysis.

\subsection{Introduction}

Dynamic models are a commonly applied tool to describe key ecological processes (Gurney and Nisbet 1998). For dynamic models to be of use in ecological studies they should be validated (Augusiak et al. 2014, Rykiel 1996), i.e. it should be evaluated whether the model provides a credible description of the ecological system it is applied to and whether it is useful for its intended purpose. Validation entails the use of objective functions (either implicitly through expert judgement, or explicitly through statistical measures) to match data to model output. For instance, matching model predictions to experimental results is a powerful way to validate models (e.g., McCauley et al. (1999)). Validation is not a one-shot exercise, but a continuous process of model evaluation, adaptation, and re-evaluation (Vemer et al. 2013). The ideal modelling cycle therefore entails an iterative loop of matching the model to (experimental) data, and using the model to generate predictions that steer new experiments for re-evaluation of the model (Fig 2.1). Usually the modelling cycle includes parameter estimation, i.e. the setting of model parameter values based on model calibration.

A common approach in analysing ecological models is by applying methods for structural stability analysis. In this approach the focus is on the asymptotic behaviour of the system, i.e. determining the asymptotic system states and their stability under different conditions (Van Nes and Scheffer 2005). Tools are available that can be used in this approach, such as MATCONT (Dhooge et al. 2003) and AUTO07P (Doedel and Oldeman 2009) for what is commonly referred to as bifurcation analysis. Structural stability or bifurcation analysis is for instance useful to assess whether models exhibit tipping points. However, for validation it is less suitable, as there is usually a large discrepancy between the time-scale for the modelled ecological system to evolve to an asymptotic state and the time-scale of validation experiments. Moreover, ecological systems seldom remain undisturbed for very long. It is therefore important for model evaluation and validation to consider the transient dynamics, i.e. the 'model behaviour that is not its final behaviour' (Hastings 2004). 


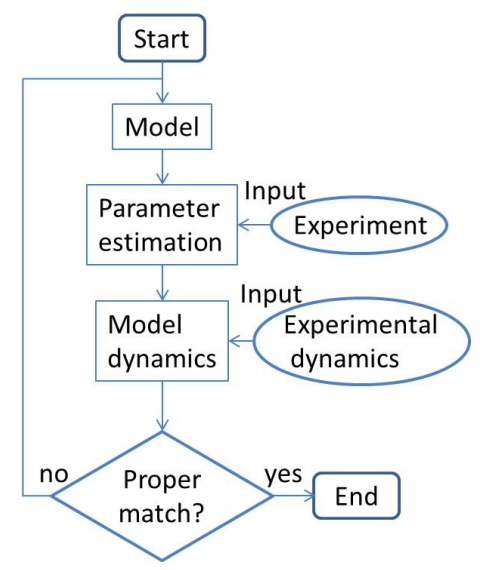

Figure 2.1: Cycle of modelling and experiment in ecology. Sensitivity analysis is important in setting up experiments to reduce a model's prediction uncertainty and in comparing model dynamics and experimental dynamics. Note that experimental dynamics should come from other experiments than those used for parameter estimation.

Methods of sensitivity analysis are well-suited to include a focus on transient dynamics. Sensitivity analysis methods have been developed and commonly applied in fields like chemistry, in which experimental circumstances are relatively well-controlled (Varma et al. 1999). In ecology it is typically more difficult to control experimental circumstances, but nevertheless the use of sensitivity analysis methods has been promoted by Cariboni et al. (2007) and Caswell (2007). Sensitivity analysis is aimed at quantifying the effects of changes or uncertainties of parameters and initial conditions on model outputs, possibly at different points in time (Saltelli et al. 2008). Typically not all model parameters contribute equally to model outputs. In other words, the model is not equally 'sensitive' to each of its parameters. If the model predictions are strongly sensitive to a certain parameter, then that parameter is a likely candidate for improved estimation. Validation efforts will then quickly reveal differences between experimental data and model predictions, and changes in these differences under parameter change. If, in contrast, the parameter has little effect on the model outcomes, then an improved estimate of that parameter will not improve the predictive power of the model. Differences between experimental data and model predictions will then not be altered strongly if the parameter is changed. In this way model parameters can be ranked in terms of sensitivity. In addition, the sensitivity of model outputs to the model parameters may vary over time. Sensitivity analysis can thus reveal what parameters and initial conditions have a considerable influence on the transient dynamics of the model, i.e. at the time-scale that is in line with that of a typical validation experiment. Sensitivity analysis can thus help ecological modellers in setting research directions and designing validation experiments, by focusing efforts on the most sensitive parameters.

Although sensitivity analysis can provide valuable information for the development of validation experiments, it depends on the selected method what kind of information is obtained. It also depends on the model analysis objective what method is 
best (Ten Broeke, Van Voorn and Ligtenberg 2016). In general, a crude distinction is made between local and global methods (Cariboni et al. 2007). Local methods target specific points in state and parameter space and thus reveal detailed information that is valid only in the neighbourhood of the targeted point. Global methods attempt to reveal statistical information that is valid over a larger region, by sampling from the full state and parameter space. The latter approach comes at a cost, namely of losing detailed information on the behaviour of the model that may be of relevance for validation.

Methods of sensitivity analysis quickly become cumbersome and time-consuming when larger models are concerned. For people for whom (statistical) model analysis is not the primary aim, or who want quick results, this is an undesirable situation that hampers the application of sensitivity analysis. The application range of sensitivity analysis methodologies is likely to be much broader if automated tools are available. In addition, automated tools are less prone to errors (assuming that the provided input is according to desired quality standards). Here we present such a tool for performing sensitivity analysis for difference equation (DE) and ordinary differential equation (ODE) models, namely GRIND for MATLAB (http://www.sparcs-center.org/grind). We demonstrate its potential by applying it to two well-known examples, namely a simple difference equation (Ricker 1954) and a classic algae-zooplankton ODE model (Rosenzweig and MacArthur 1963), and comparing its results to results obtained through semi-analytical methods.

\subsection{Local sensitivity analysis}

Here we offer a conceptual description of local sensitivity analysis. The mathematical details are given in Appendix 2.A. For the purpose of sensitivity analysis we assume that the model is a good representation of the modelled system, and there are only uncertainties regarding the parameter values, and measurement errors. Local sensitivity analysis quantifies the effect of changes in the parameters and initial conditions on the state variables. To perform local sensitivity analysis we first need to establish a default parameter set that functions as a reference point. Ideally, the default set would be the 'true' set of parameter and initial condition values, but in practice this is usually not the case. Typically we are looking for an improved parameter set and wish to know what parameters should be the focal points of further study.

Starting from the default parameter set, we consider a small change in one of the parameter values. The resulting change in the model outcomes measures the local parameter sensitivity. A large change in outcomes indicates that the parameter is influential. To rank the sensitivities, we repeat the same procedure for all model parameters and initial conditions. Note that usually not all of the model parameters have the same dimensions. To compare the sensitivities of parameters with different dimensions, they first need to be normalised, for instance by converting them to elasticities. Elasticities are dimensionless and straightforward to interpret. For instance, an elasticity of 0.24 means that a $1 \%$ change in the input parameter causes a $0.24 \%$ change in the output variable. 
Table 2.1: Symbols used throughout the text. The upper part contains general symbols, the middle part symbols for the Ricker model Eq (2.1) and the lower part for the Rosenzweig-MacArthur model Eq (2.2).

\begin{tabular}{|c|c|c|c|}
\hline Symbol & Description & Units & $\begin{array}{l}\text { Default } \\
\text { value }\end{array}$ \\
\hline$e_{i, j}$ & Elasticity of $y_{i}$ to $\phi_{j}$ & & \\
\hline $\mathbf{f} / \mathbf{F}$ & Set of ODEs/DEs & & \\
\hline$i$ & Index for state variable & & \\
\hline$j$ & Index for parameters and initial conditions & & \\
\hline$\mu$ & Total number of parameters & & \\
\hline$\nu$ & Total number of state variables & & \\
\hline $\mathbf{p} / \mathbf{P}$ & Vector of ODE/DE parameters & & \\
\hline$s_{i, j}$ & Local sensitivity of $y_{i}$ to $\phi_{j}$ & & \\
\hline$t / T$ & Time (continuous/discrete) & & \\
\hline $\mathbf{y} / \mathbf{Y}$ & Vector of state variables of ODE/DE model & & \\
\hline $\mathbf{y}_{0} / \mathbf{Y}_{0}$ & Vector of initial conditions of ODE/DE model & & \\
\hline$y_{i} / Y_{i}$ & State variable $(i=1,2, \ldots, \nu)$ of $\mathrm{ODE} / \mathrm{DE}$ model & & \\
\hline$\phi / \Phi$ & Parameters and initial conditions (ODE/DE) & & \\
\hline$\tilde{\phi} / \tilde{\boldsymbol{\Phi}}$ & Default values of $\phi / \Phi$ & & \\
\hline$c$ & Carrying capacity & $\mathrm{mg} \mathrm{l}^{-1}$ & 10 \\
\hline$N$ & Population density & $\mathrm{mg} \mathrm{l}^{-1}$ & {$[0, \rightarrow\rangle$} \\
\hline$n_{0}$ & Initial population density & $\mathrm{mg} \mathrm{l}^{-1}$ & 0.1 \\
\hline$q$ & Intraspecific growth rate & day $^{-1}$ & 0.1 \\
\hline$A$ & Density of algae & $\mathrm{mg} \mathrm{l}^{-1}$ & {$[0, \rightarrow\rangle$} \\
\hline$a_{0}$ & Initial density of algae & $\mathrm{mg} \mathrm{l}^{-1}$ & 3 \\
\hline$b$ & Efficiency of food conversion to growth & - & 0.6 \\
\hline$g$ & Maximum grazing rate of algae by zooplankton & day $^{-1}$ & 0.4 \\
\hline$h$ & Half-saturation algal concentration & $\operatorname{mg~} 1^{-1}$ & 0.6 \\
\hline$k$ & Carrying capacity & $\mathrm{mg} \mathrm{l}^{-1}$ & 10 \\
\hline$m$ & Zooplankton background mortality rate & day $^{-1}$ & 0.15 \\
\hline$r$ & Relative growth rate & day $^{-1}$ & 0.5 \\
\hline$Z$ & Density of zooplankton & $\mathrm{mg} \mathrm{l}^{-1}$ & {$[0, \rightarrow\rangle$} \\
\hline$z_{0}$ & Initial density of zooplankton & $\mathrm{mg} \mathrm{l}^{-1}$ & 3 \\
\hline
\end{tabular}




\subsubsection{Numerical approximation of local sensitivities}

Sensitivities are estimated numerically using the finite differences method. To obtain this estimate, we first run the model in the default parameter setting. We then introduce a small change in one of the parameters, and again run the model. The difference between the two runs, relative to the parameter difference, estimates the local sensitivity.

The finite differences method requires only one additional model run per parameter to estimate all of the sensitivities. The method is thus computationally rather cheap and straightforward to apply. GRIND uses the finite differences method to automatically compute all of the parameter sensitivities or elasticities.

\subsubsection{Semi-analytical calculation of local sensitivities}

To test the performance of GRIND in calculating sensitivities we use the semi-analytical method, which can be applied to DE or ODE models. In the semi-analytical method we manually, or using symbolic math software (e.g. Matlab Symbolic Math Toolbox or Maple), calculate implicit derivatives of the DEs or ODEs with respect to the model parameters. These derivatives are expressed as additional difference or differential equations, to be numerically solved alongside the original model equations. Thus, although only a single model run is needed, the semi-analytic method does require solving additional equations besides the original model equations. Since the semi-analytic method uses infinitesimal parameter differences, it yields more accurate results than the numerical method. In practice, however, it is cumbersome and prone to mistakes, especially for models with many state variables and parameters. In this paper we use the semi-analytical method to check the numerical results obtained by GRIND. Note that it is possible in GRIND to automatically perform the semi-analytic method using the Matlab Symbolic Toolbox. To illustrate the principles, we perform the method manually in this paper.

\subsection{Examples}

Below we discuss the outcomes of the sensitivity analysis for the Ricker model and for the Rosenzweig-MacArthur model. All of the results were computed in GRIND and checked semi-analytically. For both models, the results of the two methods matched closely. A step-by-step approach for the computations in GRIND and details of the semi-analytic check are given in Appendix 2.B for the Ricker model and Appendix 2.C.1 for the Rosenzweig-MacArthur model.

\subsubsection{Ricker model}

The Ricker model of population growth is given as

$$
\begin{aligned}
N\left(T+1, n_{0}, q, c\right) & =N\left(T, n_{0}, q, c\right) e^{q\left(1-\frac{N\left(T, n_{0}, q, c\right)}{c}\right)} \\
N(T=0) & =n_{0},
\end{aligned}
$$




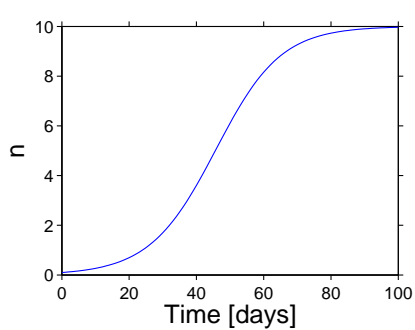

(a)

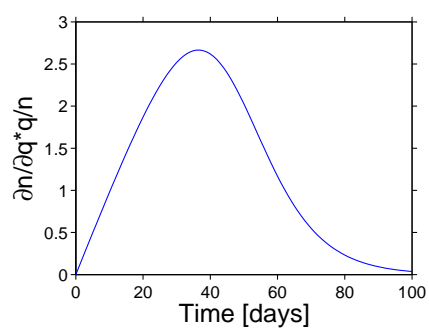

(c)

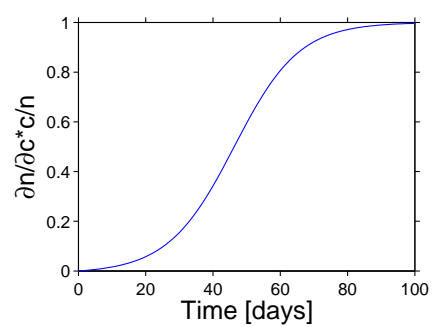

(b)

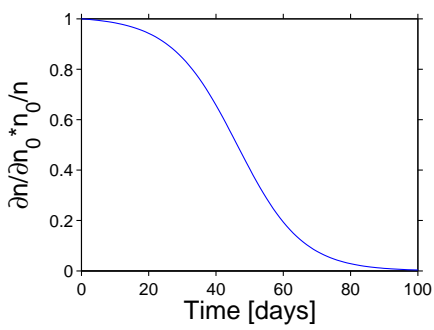

(d)

Figure 2.2: Eq (2.1a) and its elasticities in the default parameter set as a function of time, obtained with the 'timesens' command in GRIND. The elasticities show that on long time scales, $c$ is the only influential parameter. On shorter time scales $q$ and $n_{0}$ are more influential.

where $N\left(T, n_{0}, q, c\right)$ is the population density at time-step $T, q$ is the intraspecific growth factor, and $c$ is the carrying capacity. In the following we will drop the parameters from the notation and write $N(T)=N\left(T, n_{0}, q, c\right)$. A sensitivity analysis of the continuous version of the Ricker model, also known as the Pearl-Verhulst or logistic equation, has been discussed by Banks et al. (2007) and Downing et al. (2013). The population density is estimated by $N(T)$ in the default parameter set (Fig 2.2a). This population grows towards a steady state value, as is verified by setting $N(T)=$ $N(T+1)$ in Eq $(2.1 \mathrm{a})$ to determine the steady state solutions: $N=0$ and $N=c$. Analysis using GRIND shows that $N=0$ is unstable and $N=c$ is stable for any $q>0$. The model thus converges asymptotically to $c$ for any $n_{0}>0$ and $q>0$.

Initially, the solution is not sensitive to $c$, but this elasticity gradually goes to 1 as the steady state is approached (Fig 2.2). In the steady state, $c$ is the only influential parameter. In contrast, $q$ does not influence the steady state, but influences strongly the transient dynamics, especially at intermediate population sizes. The initial condition $n_{0}$ is initially the most influential parameter, but becomes less influential over time and does not affect the steady state. In experiments it may prove impossible in practical terms to monitor the ecological system up to steady state. It is therefore important to determine not only which parameters affect the asymptotic behaviour, but also which parameters are influential on short and intermediate simulation times. The sensitivity analysis shows that at different times, different parameters are the most influential ones (Fig 2.3). 


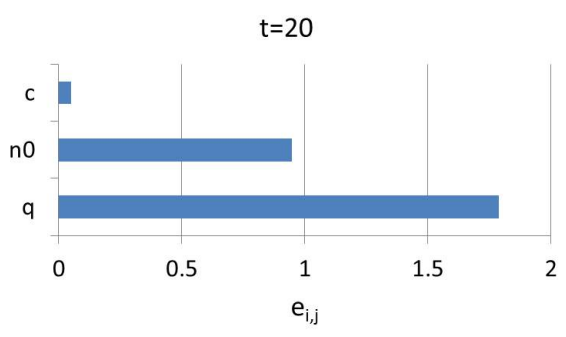

(a)

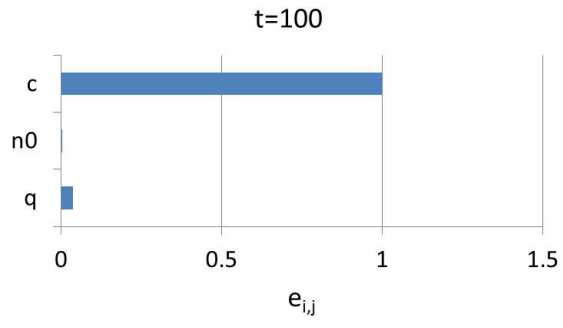

(b)

Figure 2.3: Ranking of the elasticities of Eq (2.1a) in the default parameter set at $T=20$ (a) and $T=100$ (b). The results were computed in GRIND and the graphs were made in excel. At $T=20 q$ is the most influential parameter, followed by $n_{0}$. At $T=100, c$ is the most influential and the other elasticities are close to zero.

\subsubsection{Rosenzweig-MacArthur model}

As a second example we consider the well-known Rosenzweig-MacArthur model, describing the interaction between algae and zooplankton (Rosenzweig and MacArthur 1963, Scheffer et al. 1997). The population densities depend on the initial conditions, the parameter values, and on time. To simplify the notation we will hereafter write $A=A\left(a_{0}, z_{0}, r, k, g, h, b, m, t\right)$ and similar for $Z$.

$$
\begin{aligned}
& \frac{d A}{d t}=r A\left(1-\frac{A}{k}\right)-\frac{g A}{A+h} Z \\
& \frac{d Z}{d t}=b \frac{g A}{A+h} Z-m Z \\
& A(0)=a_{0} ; \quad Z(0)=z_{0}
\end{aligned}
$$

We first consider the case where $z_{0}=0$. The model then reduces to a logistic growth equation for the algae population, which has previously been discussed by Banks et al. (2007) and Downing et al. (2013). The sensitivity analysis results (Fig 2.4) are similar to those of the Ricker model (Fig 2.2). On short time scales $r$ is the most influential parameter. The initial condition $a_{0}$ is mostly influential on smaller time scales. The carrying capacity $k$ has little influence on smaller time scales, but its elasticity grows asymptotically towards 1 , whereas the elasticities of $r$ and $a_{0}$ go to zero. Thus, the steady state is fully determined by $k$.

For $z_{0}>0$, setting $\frac{d A}{d t}=0$ and $\frac{d Z}{d t}=0$ and solving for $A$ and $Z$ yields three steady state solutions

$$
\begin{aligned}
\left(A_{1}^{*}, Z_{1}^{*}\right) & =(0,0) \\
\left(A_{2}^{*}, Z_{2}^{*}\right) & =(k, 0) \\
\left(A_{3}^{*}, Z_{3}^{*}\right) & =\left(\frac{m h}{b g-m}, \frac{b r h}{b g-m}\left(\frac{k(b g-m)-m h}{k(b g-m)}\right)\right) .
\end{aligned}
$$




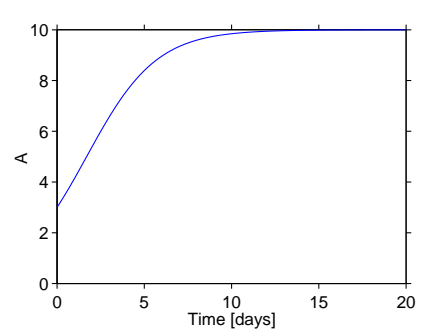

(a)

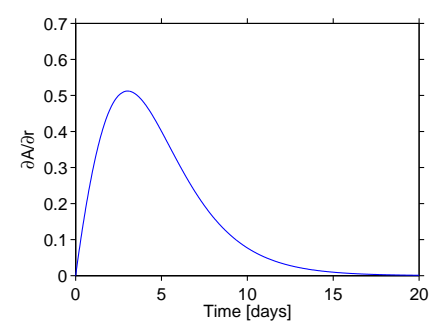

(c)

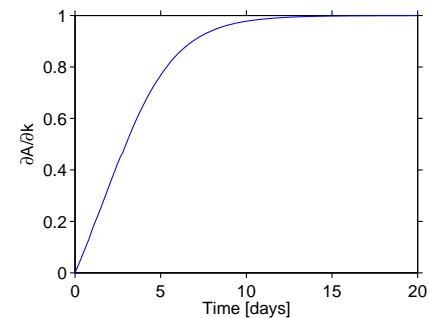

(b)

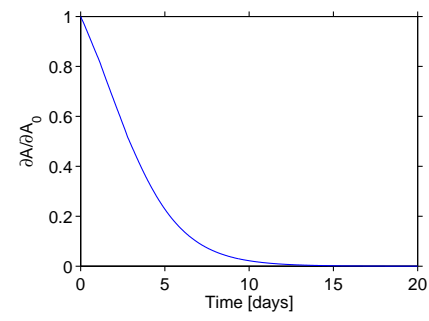

(d)

Figure 2.4: Eq (2.2a) and its local elasticities as functions of time, with $z_{0}=0$. The figures were obtained with the 'timesens' command in GRIND. In the steady state, $k$ is the only influential parameter, but for shorter simulation times $r$ and $a_{0}$ are influential.

The stability of these states is determined in GRIND by a bifurcation analysis with $k$ as bifurcation parameter and all other parameters at the default value. For small values of $k$ the zooplankton population cannot persist and goes extinct (Fig 2.5). The steady state of $\mathrm{Eq}(2.3 \mathrm{~b})$ is then stable. As $k$ increases a transcritical bifurcation is crossed, after which Eq (2.3c) is stable and the zooplankton and algae populations coexist. For even larger $k$ a Hopf bifurcation is crossed, after which both populations display limit cycles. As $k$ increases, the amplitude of the oscillations increases such that extinction of one or both populations is likely. This effect is known as the Paradox of Enrichment (Rosenzweig 1971).

In the default parameter setting the system exhibits limit cycle behaviour. Periods of relatively slow change are interrupted by periods of rapid change. Note that the sensitivities were not rescaled into elasticities because the state variables go close to zero, leading to large values for the elasticities. All of the sensitivities show peaks that follow the oscillations of the state variables (Fig 2.7). For $a_{0}$ and $z_{0}$, the amplitude of the peaks gradually decreases over time. For all other parameters the amplitude gradually increases. The peaks in the sensitivities coincide with rapid increases and decreases in $A$ and $Z$. During periods where $A$ and $Z$ change only slowly the sensitivities are much smaller. This is the case when $A$ or $Z$ is close to zero, and in the peaks where $A$ is close to carrying capacity. During these peaks, the sensitivity coefficient $\frac{\partial A}{\partial k}$ is close to 1 and the other sensitivities are close to zero. Thus, small parameter changes do not drive the peaks significantly away from carrying capacity, or the minima away from zero. During the periods where the state variables change 


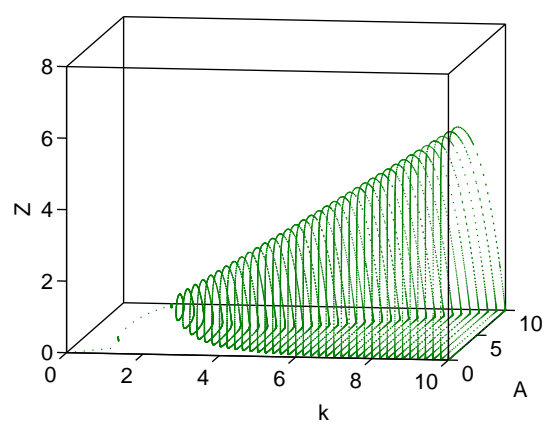

Figure 2.5: One parameter bifurcation diagram of Eq (2.2), with $k$ as bifurcation parameter. For small values of $k$ the zooplankton population goes extinct. At $k=1$ there is a transcritical bifurcation where $Z$ becomes positive. At $k=2.6$, there is a Hopf bifurcation where the steady state destabilizes and the system starts to show limit cycles. The figure was obtained with the GRIND command 'paranal'

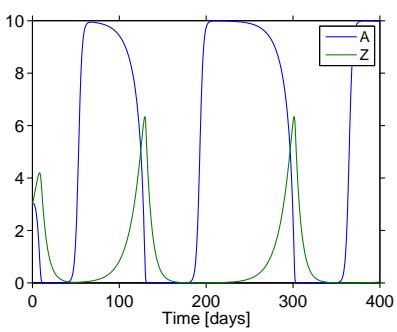

Figure 2.6: Eq (2.2) as functions of time in the default parameter set. Both state variables display limit cycles. The figure was obtained with the 'time' command in GRIND. 


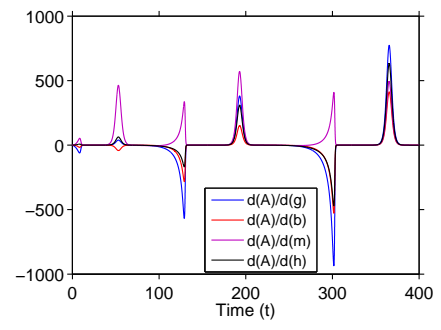

(a)

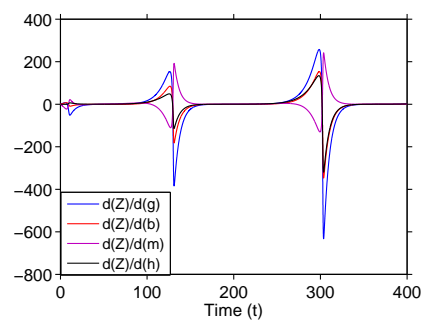

(c)

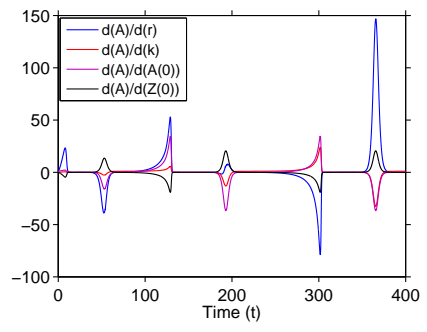

(b)

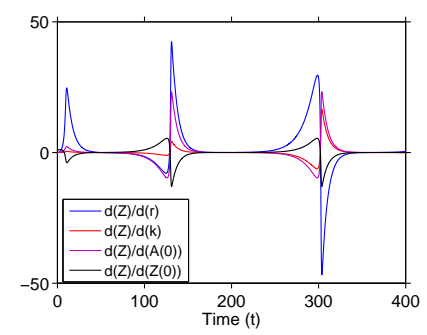

(d)

Figure 2.7: The local sensitivities of Eq (2.2) as functions of time in the default parameter set. All the sensitivities show peaks that follow the oscillations in the state variables (Fig 2.6). These figures were obtained with the GRIND command 'timesens'.

rapidly, parameter changes have a larger effect.

In this example, the ranking of influential parameters depends on the phase in the simulation. Fig 2.8 shows a ranking based on the range of the elasticities over time. Fig 2.7 shows sensitivity peaks where the parameters $g, b, h$, and $k$ are relatively influential on both $A$ and $Z$, whereas the other parameters and initial conditions are less influential. Experiments that focus on the parameters $g, b, h$, and $k$ are thus likely to yield useful information for model validation if measured at the time point where the elasticities are highest. It can be concluded that the parameters that are part of the functional response are strongly influential. For this conclusion we assume that the timing of the limit cycles is of interest. If this timing is not of interest, we may for example perform a sensitivity analysis to assess which parameters are influential on the maximum of the cycle. Furthermore, note that the ranges for the sensitivities are wide, and at other time points other parameters may be more influential. At $t=200$, for example, the ranking of influential parameters is different (Fig 2.9). The initial conditions $a_{0}$ and $z_{0}$ are still strongly influential on $A$. These elasticities become lower for larger values of $t$. For $z, m$ seems to be relatively influential even though it is the least influential parameter in Fig 2.8. 


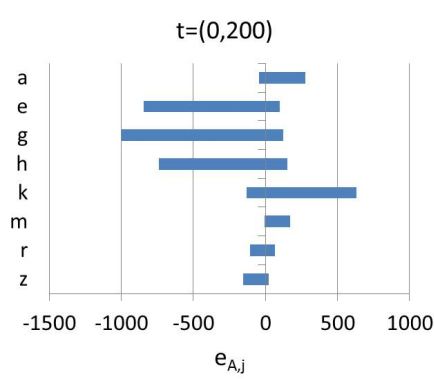

(a)

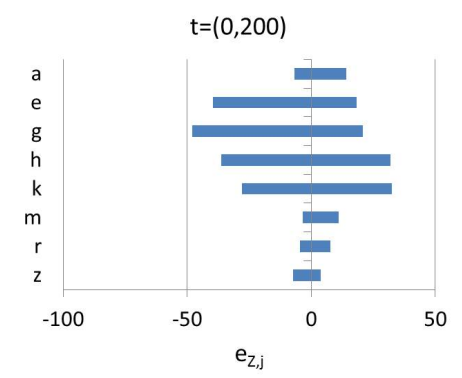

(b)

Figure 2.8: Range of the elasticities of $A$ (a) and $Z$ (b) between $t=0$ and $t=400$. The parameters are ordered by the wideness of the range of the elasticity, from widest at the top to narrowest at the bottom. Based on this ordering $g$ can be considered the most influential parameter. The maxima and minima correspond to the peaks in Fig 2.7. At other time points the sensitivity ranking may be different (e.g. Fig 2.9). The results were obtained in GRIND and displayed using Excel.

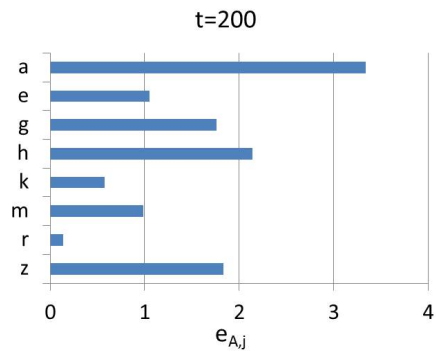

(a)

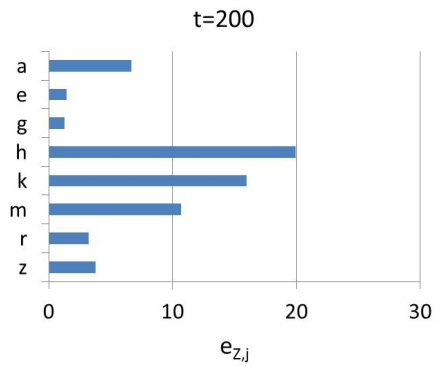

(b)

Figure 2.9: Absolute values of the elasticities of $A(\mathrm{a})$ and $Z$ (b) at $t=200$. This ordering shows which parameters are the most influential at $t=200$. The ordering differs from Fig 2.8. The results were obtained in GRIND and displayed using Excel.

\subsection{Discussion}

In this paper we have shown how to carry out local sensitivity analysis for ecological models with the tool GRIND for MATLAB. We illustrate the functionality and performance of the tool by two examples that are based on well-known ecological models, namely the Ricker model and the Rosenzweig-MacArthur model. The performance of the tool is demonstrated by a comparison of results obtained with the tool to results obtained with a semi-analytical method. We find that the results obtained by using the two methods are in close agreement. The tool can hence be used for a fast and user-friendly local sensitivity analysis of ecological models. This provide an attractive means for ecologists who want to use sensitivity analysis in the process of model validation.

Local sensitivity analysis considers only small parameter changes relative to the 
default point. Some authors therefore recommend to use global methods (e.g. Cariboni et al. (2007)), which quantify sensitivities over a larger region of the parameter space. The aggregation of model outcomes from various samples across a region of state and/or parameter space into sample statistics can, however, obscure characteristics of the underlying model behaviour that are relevant for model validation experiments (Rakovec et al. 2014), such as the existence of tipping points (van Nes and Scheffer 2003, Ten Broeke, Van Voorn and Ligtenberg 2016, Ten Broeke, Van Voorn, Kooi and Molenaar 2016). For example, local sensitivity analysis of the RosenzweigMacArthur model gives different outcomes for a parameter setting in which the state variables converge asymptotically to a positive steady state (Fig 2.7), or to a state where the predator is extinct (Fig 2.4). To understand the model behaviour, these cases should be treated separately, rather than aggregated into a single global sensitivity measure.

By identifying which parameters are influential at certain time points during the simulation, local sensitivity analysis can yield information that is useful for guiding the design of experiments for model parametrization and validation. For instance, for the Rosenzweig-MacArthur model our results show that the parameters $g, b, h$, and $k$ strongly influence both state variables during peaks in the sensitivities, whereas other parameters have a much smaller effect. Thus, experiments that focus on these four parameters and that measure the system during those peaks are likely to yield valuable information for model validation. Experiments that focus on other parameters or on different time points do not reveal the same level of detailed information, because less influential parameters do not affect the outcomes as much and hence less can be concluded about (mis)matches between experimental data and model predictions. Our study thus shows that GRIND helps to obtain useful information for the design of validation experiments in a user-friendly way.

\section{Acknowledgements}

We thank J. Grasman for comments on an earlier version of the manuscript. The research by GTB was financed by the IO/OP theme Complex Adaptive Systems at Wageningen University

\section{A Local sensitivity analysis}

We will consider local sensitivity analysis for two types of dynamic equations that are commonly used to describe rates of change in ecological processes. These are ordinary differential equations (ODEs) and difference equations (DEs). ODEs are given as

$$
\frac{d \mathbf{y}(t)}{d t}=\mathbf{f}(\mathbf{y}(t), \mathbf{p}, t)
$$

and DEs as

$$
\mathbf{Y}(T+1)=\mathbf{F}(\mathbf{Y}(T), \mathbf{P}, T)
$$


where $t$ denotes continuous time, and $T$ denotes discrete time. The vector $\mathbf{y}$ respectively $\mathbf{Y}$ contains state variables, for instance population densities or substance concentrations. The vector $\mathbf{p}$ respectively $\mathbf{P}$ contains parameter values (e.g. intraspecific growth rate, or carrying capacity). To solve these equations algebraically or numerically initial conditions are required (e.g. the population density at the start time of the experiment), given for ODEs as

$$
\mathbf{y}(0)=\mathbf{y}_{0}
$$

and for DEs as

$$
\mathbf{Y}(0)=\mathbf{Y}_{0}
$$

For sensitivity analysis we want to express the response of the model to variations in its parameters or initial conditions. We denote the vector containing parameters and initial conditions as $\boldsymbol{\phi}=\left(\mathbf{p}, \mathbf{y}_{\mathbf{0}}\right)$ for ODEs and as $\boldsymbol{\Phi}=\left(\mathbf{P}, \mathbf{Y}_{\mathbf{0}}\right)$ for DEs. A small change in any of the parameters or initial conditions to $\widetilde{\phi}_{j}+\Delta \phi_{j}$ corresponds to a new solution $y_{i}\left(\widetilde{\phi}_{j}+\Delta \phi_{j}, t\right)$, where the unchanged parameters were omitted from the notation. The index $i(i=1,2, \ldots, \nu)$ can denote any of the state variables, with $\nu$ the total number of state variables, while index $j(j=1,2, \ldots, \mu+\nu)$ can denote any of the parameters or initial conditions, with $\mu$ the number of parameters. Assuming $\Delta \phi_{j}$ to be sufficiently small, we estimate the solution with a first order Taylor approximation around the default solution

$$
\frac{y_{i}\left(\widetilde{\phi}_{j}+\Delta \phi_{j}, t\right)-y_{i}\left(\widetilde{\phi}_{j}, t\right)}{\Delta \phi_{j}} \approx \frac{\partial y_{i}(t)}{\partial \phi_{j}} .
$$

Replacing the small letters with capitals, the same expression holds for difference equations. The right hand side of Eq (2.8) is the local sensitivity coefficient $s_{i, j}$ (Varma et al. 1999)

$$
s_{i, j}(t)=\frac{\partial y_{i}(t)}{\partial \phi_{j}}
$$

which can be evaluated at any time $t$. If the value of $s_{i, j}(t)$ is large, the parameter change strongly affects the solution. If the value is small, the parameter change has little effect.

A drawback of the above local sensitivity coefficient for experimental purposes is that it is not dimensionless. Hence, it is not straightforward to compare local sensitivities of parameters with different dimensions. A dimensionless alternative is the elasticity, which is obtained by rescaling Eq (2.9)

$$
e_{i, j}(t)=\frac{\partial y_{i}(t)}{\partial \phi_{j}} \frac{\phi_{j}}{y_{i}(t)}
$$


Elasticities may be used to rank parameters in terms of how strongly a relative change in the parameter value affects the model outcomes. For instance, an elasticity of 5 means that a $1 \%$ change in the input parameter causes a $5 \%$ change in the output variable. Note that the state variable that is used to scale the elasticity is a function of time. When comparing elasticities that were computed at different simulation times, it should thus be considered that the scaling factors are different.

\section{A.1 Numerical approximation of local sensitivities}

GRIND uses the finite differences method to approximate local sensitivities, based on Eq (2.8). This method has the advantage of not having to solve any additional equations. If the default solution has been computed, the finite differences method requires only one additional model run per sensitivity in order to determine $y_{i}\left(\phi_{j}+\right.$ $\left.\Delta \phi_{j}, t\right)$. The sensitivity is then directly computed from the difference between the two solutions, as in Eq (2.8).

To accurately estimate the local sensitivity, a small value for $\Delta \phi_{i}$ should be used. To achieve a sufficient level of accuracy GRIND makes use of the Runge Kutta solver of Matlab (ODE45, with a absolute and relative error of $10^{-11}$, Shampine and Reichelt (1997)). By default GRIND uses for $\Delta \phi_{i}$ a relative disturbance of $10^{-8}$ times the original value.

\section{A.2 Semi-analytical calculation of local sensitivities}

To test the performance of GRIND in calculating sensitivities we use of the semianalytical method. Consider the partial derivative of Eq (2.4) or Eq (2.5) to parameter $\phi_{j}$

$$
\frac{d}{d t}\left(\frac{\partial y_{i}\left(t, \phi_{j}\right)}{\partial \phi_{j}}\right)=\frac{\partial f\left(\mathbf{y}\left(t, \phi_{j}\right), \phi_{j}\right)}{\partial \phi_{j}},
$$

or for difference equations,

$$
\frac{\partial Y_{i}\left(T+1, \Phi_{j}\right)}{\partial \Phi_{j}}=\frac{\partial F\left(\mathbf{Y}\left(T, \Phi_{j}\right), \Phi_{j}\right)}{\partial \Phi_{j}}
$$

The state variables $y(t)$ respectively $Y(T)$ depend not only on time but also on the parameter values. Manually computing the partial derivatives gives an additional dynamic equation for every state variable. The resulting set of equations is expressed as

$$
\frac{d}{d t}\left(s_{i, j}\right)=\frac{\partial f_{i}}{\partial \phi_{j}}+\sum_{m=1}^{\nu} \frac{\partial f_{i}}{\partial y_{m}} s_{m, j}
$$

and equivalent for difference equations. At $t=0$ or $T=0$, the state variables are fully given by the initial conditions and do not depend on the parameters. The initial conditions are thus 


$$
s_{i, j}(0)= \begin{cases}1 & \text { if } \phi_{j} \text { is the initial condition of } y_{i} \\ 0 & \text { else, }\end{cases}
$$

and equivalent for difference equations. The equations for the sensitivities are then solved alongside the original model equations. This amounts to a total number of $\nu \times(\mu+\nu)$ additional equations for all of the sensitivities. The resulting coupled non-linear model can be solved numerically, hence why we refer to this approach as semi-analytical.

\section{B Ricker model}

\section{B.1 Numerical sensitivity analysis in GRIND}

We perform a local sensitivity analysis of the Ricker model in GRIND. The model consists of the difference equation

$$
N\left(t+1, n_{0}, q, c\right)=N\left(t, n_{0}, q, c\right) e^{q\left(1-\frac{N\left(t, n_{0}, q, c\right)}{c}\right)},
$$

with initial condition

$$
N(0)=n_{0}
$$

with $N\left(t, n_{0}, q, c\right)$ the population density at time-step $t, q$ the intraspecific growth factor, and $c$ the carrying capacity (Table 2.1). From now on we write $N(t)=$ $N\left(t, n_{0}, q, c\right)$ and drop the parameters from the notation. After installing GRIND, it is opened by typing 'model' in the command window in Matlab. A window is opened containing two text boxes. The model equation is typed in the upper box (Fig 2.10). The lower box contains the values for the initial condition and the parameters. Clicking 'ok' loads the model and returns to the command window, where we can now use GRIND commands.

The 'time' command displays the population density $N(t)$ as a function of time (Fig 2.11). The solution converges asymptotically towards $c$. This is confirmed by an iteration map in which the population size at the next time step $N(t+1)$ is plotted as function of the current population size $N(t)$. To draw the iteration map we first type 'ax $\times \mathrm{n}[0,20]$ ' to set the state variable and its range on the horizontal axis. We then use the 'null' command to display the iteration map (Fig 2.12). The intersections in the iteration map correspond to the steady states of the model $N_{1}=0$ and $N_{2}=10$. Clicking anywhere in the figure and choosing the option 'Run forward' displays a model run starting from the corresponding population density. Fig 2.12 shows one run starting from a low population density and a run starting from a high population density. Both runs converge towards the positive steady state $N_{2}$. Thus, $N_{2}$ is stable and $N_{1}$ is unstable at the default parameter values.

The parameter sensitivities are obtained using the 'timesens' command. We choose to compute the elasticity coefficients instead of the sensitivities by typing ' $\mathrm{Y}$ ' under 


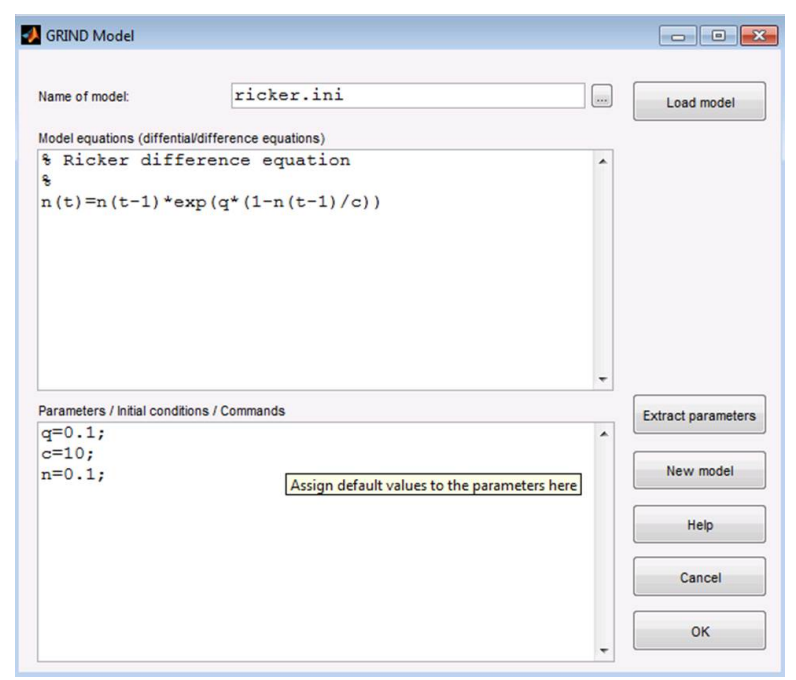

Figure 2.10: The GRIND model window is opened by typing 'model' in the Matlab command window. The upper text box contains the model equations. The lower text box contains the values of the parameters and initial conditions.

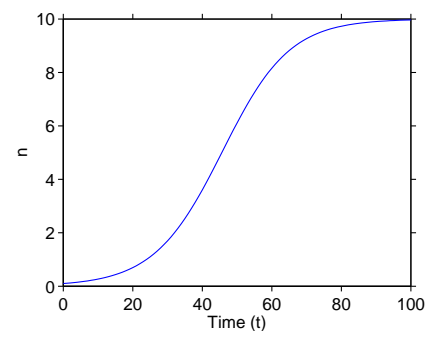

Figure 2.11: $N(t)(\mathrm{Eq}(2.15))$ as a function of time in the default parameter set. The population grows towards the carrying capacity $c$. The figure was obtained with the GRIND command 'time'.

the option 'Elasticity'. We leave all other options on default. The elasticities are computed after clicking 'ok'. Using the 'time' command now displays the elasticities as a function of time (Fig 2.13). On shorter time scales, the growth rate $q$ and the initial condition $n_{0}$ are influential. On longer time scales the elasticity coefficients of $q$ and $n_{0}$ decrease, whereas the elasticity coefficient of $c$ asymptotically grows to 1 . Thus, the final model behaviour is completely determined by $c$, but $q$ and $n_{0}$ are more important on shorter time scales.

\section{B.2 Semi-analytical sensitivity analysis}

We check the results of the local sensitivity analysis in GRIND semi-analytically. We manually calculate the derivatives of $N(t+1)(\mathrm{Eq}(2.15))$ to the parameters and initial condition. Since $N$ is also a function of the parameters and initial condition as well 


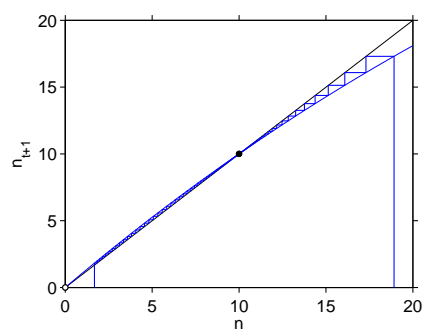

Figure 2.12: Iteration map of $\mathrm{Eq}(2.15)$ in the default parameter set.The intersections in the origin and at $N(t)=c$ correspond to steady states. The figure was obtained with the GRIND command 'null'. Single runs are shown by clicking a starting point and choosing the 'run forward' command. Two model runs with different initial conditions converge to $c$. Thus, the steady state at $N(t)=c$ is stable and the steady state at $N(t)=0$ is not stable.

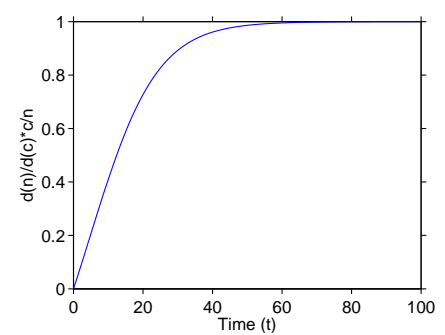

(a)

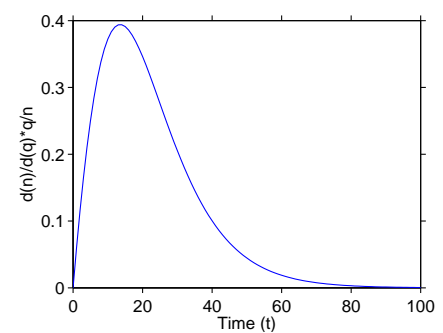

(b)

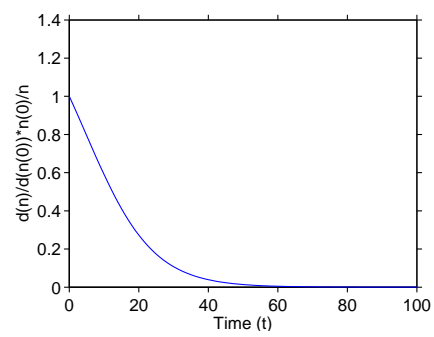

(c)

Figure 2.13: The elasticities of Eq (2.15) as functions of time in the default parameter set. The figures were obtained with the GRIND command 'timesens' to compute the results, and the command 'time' to display the figures. The elasticity of $c$ (a) goes to 1 for large $t$. Thus, the parameter $c$ fully determines the long-term model outcome. For shorter simulation times, however, the other parameters are more influential. 
Table 2.2: Differences between the sensitivities that were computed in GRIND and the semianalytical results, relative to the value of the state variable $N$.

\begin{tabular}{l|lll} 
parameter & $q$ & $c$ & $n_{0}$ \\
\hline relative difference [\%] & $7.2 \cdot 10^{-5}$ & $8.0 \cdot 10^{-4}$ & $1.1 \cdot 10^{-3}$
\end{tabular}

as time, we apply the method of implicit differentiation to obtain

$$
\begin{aligned}
& \frac{\partial N}{\partial q}(t+1)=\frac{\partial N}{\partial q}(t) e^{q\left(1-\frac{N(t)}{c}\right)}+N(t) \frac{\partial}{\partial q}\left(e^{q\left(1-\frac{N(t)}{c}\right)}\right) \\
& \frac{\partial N}{\partial c}(t+1)=\frac{\partial N}{\partial c}(t) e^{q\left(1-\frac{N(t)}{c}\right)}+N(t) \frac{\partial}{\partial c}\left(e^{q\left(1-\frac{N(t)}{c}\right)}\right) \\
& \frac{\partial N}{\partial n_{0}}(t+1)=\frac{\partial N}{\partial n_{0}}(t) e^{q\left(1-\frac{N(t)}{c}\right)}+N(t) \frac{\partial}{\partial n_{0}}\left(e^{q\left(1-\frac{N(t)}{c}\right)}\right)
\end{aligned}
$$

The derivatives of the exponentials are further worked out using the chain rule

$$
\begin{aligned}
& \frac{\partial N}{\partial q}(t+1)=\left[\left(1-\frac{q}{c} N(t)\right) \frac{\partial N}{\partial q}(t)+N(t)\left(1-\frac{N(t)}{c}\right)\right] e^{q\left(1-\frac{N(t)}{c}\right)} \\
& \frac{\partial N}{\partial c}(t+1)=\left[\frac{\partial N}{\partial c}(t)+\frac{q}{c^{2}} N^{2}(t)-\frac{q}{c} N(t) \frac{\partial N}{\partial c}(t)\right] e^{q\left(1-\frac{N(t)}{c}\right)} \\
& \frac{\partial N}{\partial n_{0}}(t+1)=\left(1-\frac{q}{c} N(t)\right) \frac{\partial N}{\partial n_{0}}(t) e^{q\left(1-\frac{N(t)}{c}\right)} .
\end{aligned}
$$

At $t=0$, we have $N(0)=n_{0}$. The initial conditions are thus

$$
\frac{\partial N}{\partial q}(0)=0 ; \quad \frac{\partial N}{\partial c}(0)=0 ; \quad \frac{\partial N}{\partial n_{0}}(0)=1 .
$$

This set of differential equations was solved in GRIND alongside Eq (2.1a). The resulting sensitivities were rescaled to elasticities (Eq (2.10)) and compared to the results obtained in GRIND. The differences between the two methods are acceptably small (Table (2.2)). Note that the semi-analytical results may also be obtained in GRIND using the command 'timesens -sym par1 par2', where for par1and par2 parameter names are inserted. The results using this command are in agreement with those reported here.

\section{C Rosenzweig-MacArthur model}

\section{C.1 Numerical sensitivity analysis in GRIND}

We perform a numerical sensitivity analysis in GRIND for the well-known RosenzweigMacArthur model, describing the interaction between algae and zooplankton (Rosenzweig and MacArthur 1963, Scheffer et al. 1997). 


$$
\begin{aligned}
& \frac{d A}{d t}=r A\left(1-\frac{A}{k}\right)-\frac{g A}{A+h} Z \\
& \frac{d Z}{d t}=b \frac{g A}{A+h} Z-m Z \\
& A(0)=a_{0} ; \quad Z(0)=z_{0}
\end{aligned}
$$

Setting $\frac{d A}{d t}=0$ and $\frac{d Z}{d t}=0$ and solving for $A$ and $Z$ yields three steady states

$$
\begin{aligned}
(A, Z) & =(0,0) \\
(A, Z) & =(k, 0) \\
(A, Z) & =\left(\frac{m h}{b g-m}, \frac{b r h}{b g-m}\left(\frac{k(b g-m)-m h}{k(b g-m)}\right)\right) .
\end{aligned}
$$

The stability of these states is shown in GRIND by a bifurcation analysis with $k$ as bifurcation parameter. We first write the model equations in GRIND (Fig 2.14) and then type 'paranal' in the Matlab command window. We then select $k$ as bifurcation parameter and leave all other options on default (Fig 2.15). The resulting plot shows that for small $k$ the second equilibrium of $\mathrm{Eq}(2.21)$ is stable and the zooplankton population thus goes extinct. As $k$ is increased a transcritical bifurcation is crossed, after which the zooplankton and algae coexist. For even larger $k$ a Hopf bifurcation is crossed, after which the two populations display periodic behaviour. As $k$ increases, the amplitude of the oscillations increases such that extinction of one or both populations is likely. This effect is known as the Paradox of Enrichment (Rosenzweig 1971).

All the sensitivities were computed in GRIND. We first consider the case where $z_{0}=0$. The model then reduces to a logistic growth equation for the algae population. A sensitivity analysis for the logistic growth model was discussed by Banks et al. (2007) and Downing et al. (2013). To perform the analysis in GRIND, we first type 'model' and set $z_{0}=0$. We type 'simtime 020 ' to set the time interval for the computations from $t=0$ to $t=20$. Typing 'time' in the MatLaB command window shows $A$ as a function of time (Fig 2.17a) and $Z=0$. $A$ grows logistically towards $k$. The 'timesens' command computes the sensitivity coefficients. We type 'Y' under the option to compute the elasticity and leave all other options on default. Under 'parameters', we list $k, r$ and $A$ (in GRIND this refers to the initial condition $a_{0}$ ). Since $Z=0$, the other parameters have no effect. The 'time' command now displays the elasticity coefficients as functions of time (Fig 2.17). On shorter time scales $r$ is the most influential parameter. The initial condition $a_{0}$ is influential mostly on smaller time scales. The carrying capacity $k$ has little influence on smaller time scales. On longer time scales, however, the elasticity coefficient of $k$ grows towards 1 , whereas the elasticities of $r$ and $a_{0}$ go to zero. Thus, the asymptotic behaviour is fully determined by $k$.

To show how the sensitivities are affected by limit cycles, we carry out the sensitivity analysis both for $k=1.5$, where the model evolves to a steady state of coexistence, and for the default value $k=10$, where the model displays limit cycles. Note that we 


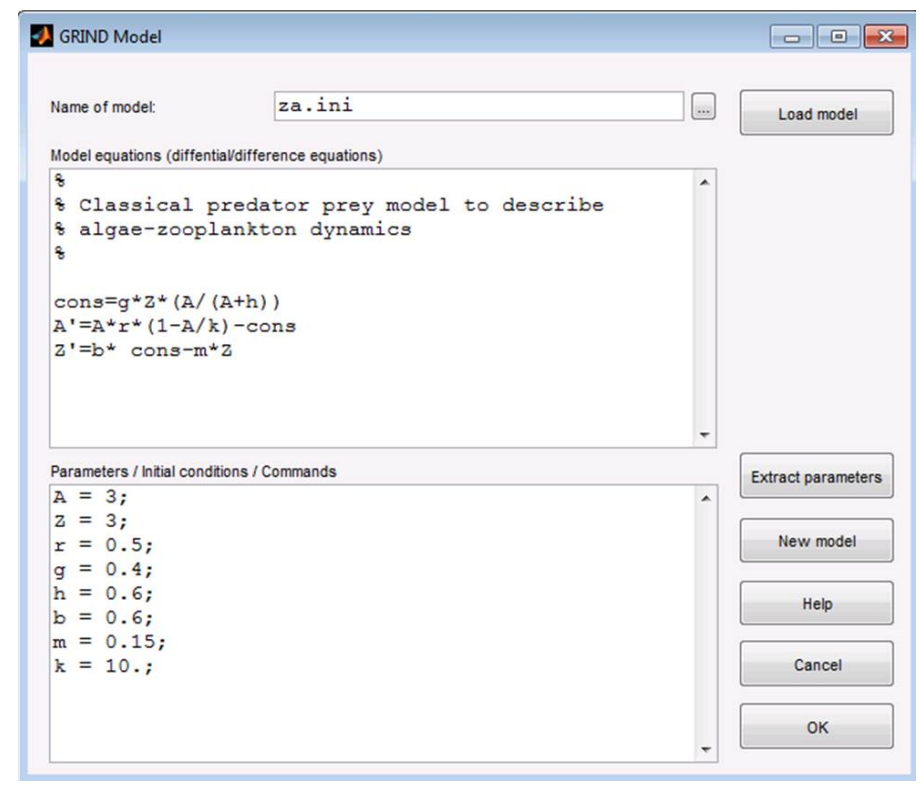

Figure 2.14: The differential equations for the model (Eq 2.20). The lower text box contains the initial conditions and parameter values.

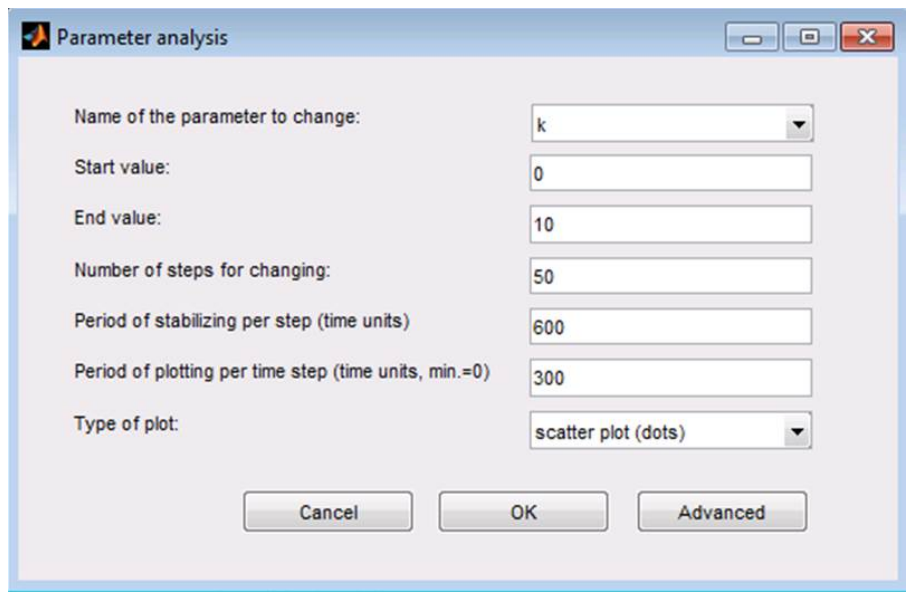

Figure 2.15: To perform a bifurcation analysis of the Rosenzweig-MacArthur model in GRIND, we select $k$ as bifurcation parameter and leave all other option on default. 


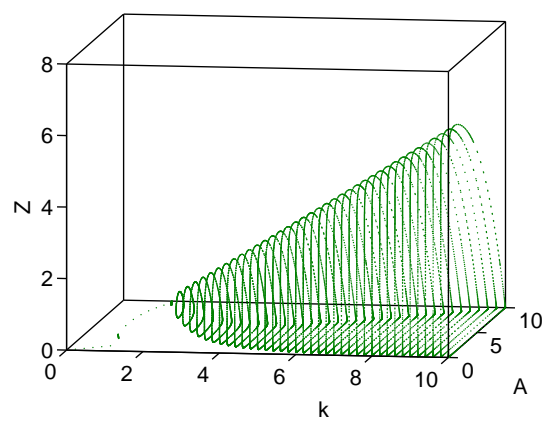

Figure 2.16: One parameter bifurcation diagram of Eq (2.20), with $k$ as bifurcation parameter. There is a transcritical bifurcation at $k=1$ where $Z$ becomes positive. At $k=2.6$, there is a Hopf bifurcation where the steady state destabilizes and the system starts showing limit cycles. The figure was obtained with the GRIND command 'paranal'

Table 2.3: The differences between the numerical and the semi-analytical results for the sensitivity coefficients were computed at every time-step. We show the maximum value over time for all the combinations of state variables and parameters. The results in GRIND closely match the semi-analytical results.

\begin{tabular}{l|ll}
\hline & \multicolumn{2}{|c}{ Deviation } \\
& $A$ & $Z$ \\
\hline$r$ & $2.1 \cdot 10^{-3}$ & $2.1 \cdot 10^{-4}$ \\
$k$ & $3.6 \cdot 10^{-3}$ & $3.4 \cdot 10^{-3}$ \\
$g$ & $1.5 \cdot 10^{-2}$ & $5.8 \cdot 10^{-4}$ \\
$h$ & $1.2 \cdot 10^{-3}$ & $1.0 \cdot 10^{-4}$ \\
$b$ & $1.1 \cdot 10^{-3}$ & $4.4 \cdot 10^{-2}$ \\
$m$ & $1.1 \cdot 10^{-3}$ & $1.1 \cdot 10^{-4}$ \\
$a_{0}$ & $1.2 \cdot 10^{-3}$ & $1.5 \cdot 10^{-4}$ \\
$z_{0}$ & $3.7 \cdot 10^{-3}$ & $2.2 \cdot 10^{-4}$
\end{tabular}




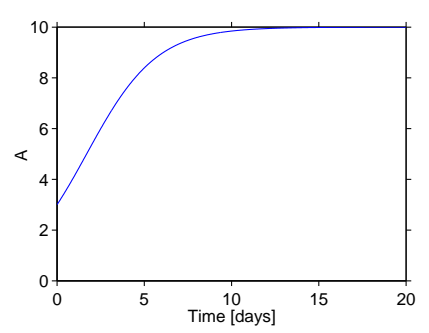

(a)

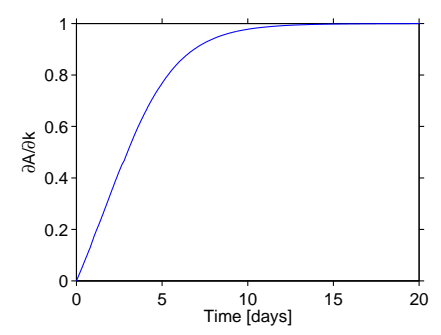

(c)

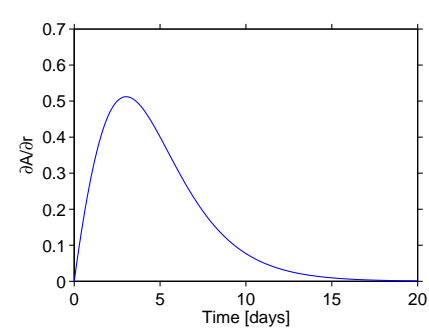

(b)

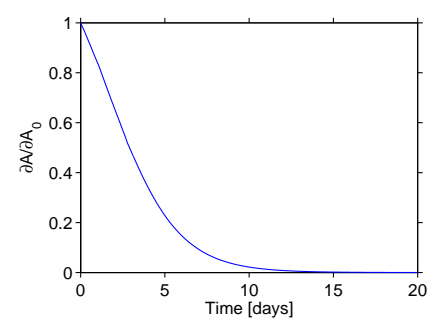

(d)

Figure 2.17: Eq (2.20a) and its local elasticities as functions of time, with $z_{0}=0$. The figures were obtained with the 'timesens' command in GRIND, followed by the 'time' command to display the figures.

have not rescaled the resulting sensitivities into elasticities because $A$ and $Z$ in some cases go close to zero, leading to large values for the elasticities. We type 'model' and set $k=1.5$. We set the time interval from $t=0$ to $t=400$ by typing 'simtime 0400 4000'. The 4000 indicates the number of output points and ensures that there is a set time-step for the output values. Manually setting a time-step that is sufficiently small can improve the accuracy of sensitivity estimates in GRIND. We then compute the sensitivities using the 'timesens' command, including all of the parameters and initial conditions. The sensitivities are shown in Fig 2.19. As $A$ and $Z$ reach the steady state values, the parameter sensitivities also converge towards constant values. The sensitivities to the initial conditions go to zero. Furthermore, the sensitivities $\frac{\partial A}{\partial r}$ and $\frac{\partial A}{\partial k}$ also go to zero. That $\frac{\partial A}{\partial k}=0$ may seem surprising given the results for the Ricker model. However, Eq (2.3c) shows that the steady state value of $A$ does not depend on $k$ or $r$. All other sensitivities have nonzero steady state values and thus affect the asymptotic behaviour.

On short time scales both state variables are to some extent sensitive to all parameters and initial conditions. There is a peak in the sensitivities of $A$ around $t=30$, which coincides with a strong increase in the value of $A$. Another peak follows somewhat before $t=200$, where $A$ is converging to its steady state value, but this is not as clear for all of the parameters. For $Z$, a first peak in the sensitivities occurs close $t=0$, coinciding with a strong decrease of $Z$. A second peak occurs around $t=180$, while $Z$ gradually increases towards the steady state. These results thus show peaks where the output is strongly sensitive to some parameters. Measurements made during the peaks of the sensitivity of that parameter would help to reduce the 


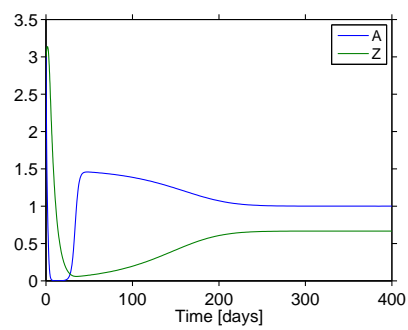

Figure 2.18: Eq (2.20) as a function of time with $k=1.5$ and all other parameters at their default values. The figure was obtained with the GRIND command 'time'.

uncertainty of $A$ and $Z$ by estimating the parameter more accurately.

For $k=10$, the model shows limit cycles (Fig 2.20). Times where the state variables remain approximately constant are interrupted by times of rapid change. The sensitivities are computed in the same way as for $k=1.5$. All of the sensitivities show peaks that follow the oscillations of the state variables (Fig 2.21). It was checked that for $a_{0}$ and $z_{0}$, the amplitude of the peaks decreases gradually over time. The amplitudes for all other sensitivities gradually increase. When $A$ is close to zero, its sensitivities are also close to zero. Small parameter changes thus do not prevent the population from being driven close to extinction. The peaks of $A$ reach close to carrying capacity $k$. During those peaks, the sensitivity coefficient $\frac{\partial A}{\partial k}$ lies close to 1 and the other sensitivities are close to zero. This means that small parameter changes do not drive the peaks significantly away from carrying capacity. When $Z$ is close to zero, its sensitivities are also close to zero. For both $A$ and $Z$, the timing of the peaks in the sensitivities coincides with increases and decreases in $A$ and $Z$.

\section{C.2 Semi-analytical sensitivity analysis}

We verify the computation of the sensitivity coefficients in GRIND using the semianalytic method. Manually taking the derivatives of Eq (2.20) to model parameters and initial conditions yields analytical expressions for the 16 sensitivity coefficients. 


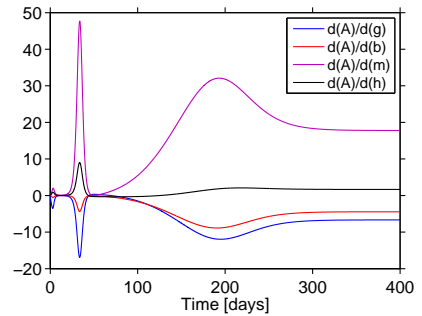

(a)

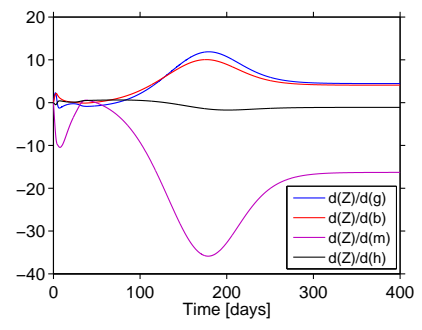

(c)

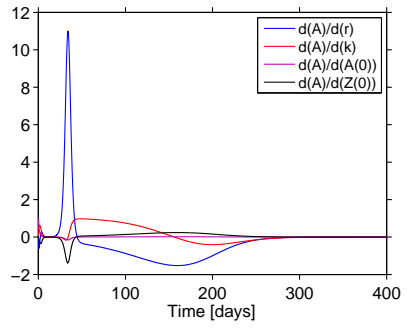

(b)

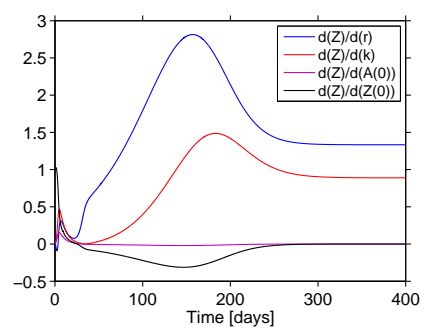

$(d)$

Figure 2.19: The local sensitivities of Eq (2.20) as a function of time with $k=1.5$ and all other parameters at their default values. These figures were obtained with the GRIND command 'timesens' to compute the results, and the command 'time' to display the figures. Note that by default GRIND returns a separate plot for each elasticity. The graphs were manually combined to display the information in a more condensed form.

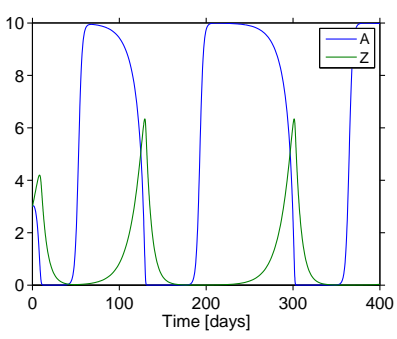

Figure 2.20: Eq (2.20) as functions of time in the default parameter set. The figure was obtained with the 'time' command in GRIND. 


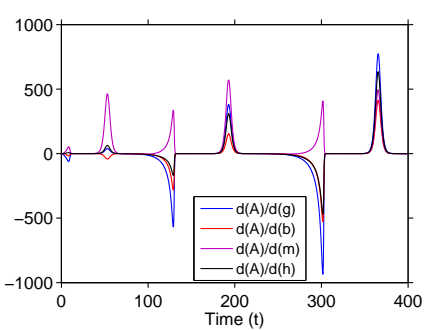

(a)

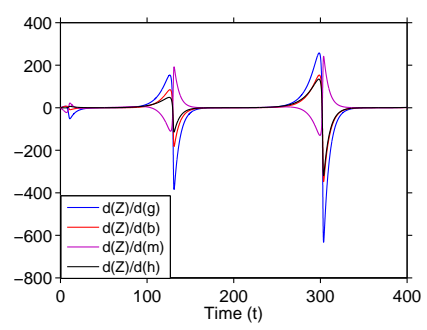

(c)

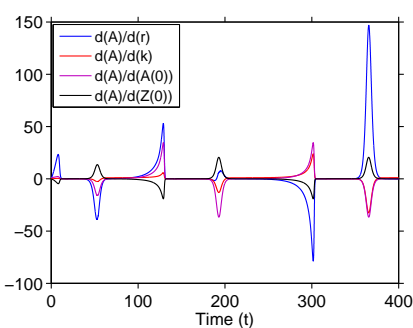

(b)

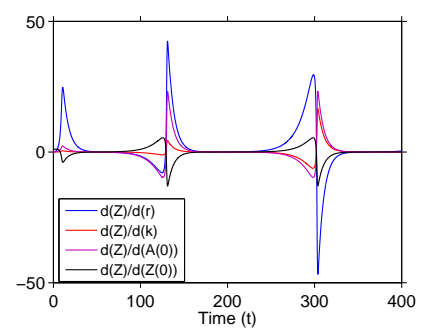

(d)

Figure 2.21: The local sensitivities of Eq (2.20) as functions of time in the default parameter set. These figures were obtained with the GRIND command 'timesens' to compute the results, and the command 'time' to display the figures. Note that by default GRIND returns a separate plot for each elasticity. The graphs were manually combined to display the information in a more condensed form. 


$$
\begin{aligned}
& \frac{d}{d t}\left(\frac{\partial A}{\partial r}\right)=A\left(1-\frac{A}{K}\right)+r\left(1-\frac{2 A}{K}\right) \frac{\partial A}{\partial r}-\frac{g Z h}{(A+h)^{2}} \frac{\partial A}{\partial r}-\frac{g A}{A+h} \frac{\partial Z}{\partial r} \\
& \frac{d}{d t}\left(\frac{\partial Z}{\partial r}\right)=\frac{b g h Z}{(A+h)^{2}} \frac{\partial A}{\partial r}+\left(\frac{b g A}{A+h}-m\right) \frac{\partial Z}{\partial r} \\
& \frac{d}{d t}\left(\frac{\partial A}{\partial K}\right)=r\left(1-2 \frac{A}{K}\right) \frac{\partial A}{\partial K}+\frac{r A^{2}}{K^{2}}-\frac{g Z h}{(A+h)^{2}} \frac{\partial A}{\partial K}-\frac{g A}{A+h} \frac{\partial Z}{\partial K} \\
& \frac{d}{d t}\left(\frac{\partial Z}{\partial K}\right)=\frac{b g Z h}{(A+h)^{2}} \frac{\partial A}{\partial K}+\left(\frac{b g A}{A+h}-m\right) \frac{\partial Z}{\partial K} \\
& \frac{d}{d t}\left(\frac{\partial A}{\partial g}\right)=r\left(1-\frac{2 A}{K}\right) \frac{\partial A}{\partial g}-\frac{A Z}{A+h}-\frac{g Z h}{(A+h)^{2}} \frac{\partial A}{\partial g}-\frac{g A}{A+h} \frac{\partial Z}{\partial g} \\
& \frac{d}{d t}\left(\frac{\partial Z}{\partial g}\right)=\frac{b A Z}{A+h}+\frac{b g Z h}{(A+h)^{2}} \frac{\partial A}{\partial g}+\left(\frac{b g A}{A+h}-m\right) \frac{\partial Z}{\partial g} \\
& \frac{d}{d t}\left(\frac{\partial A}{\partial h}\right)=r\left(1-\frac{2 A}{K}\right) \frac{\partial A}{\partial h}+\frac{g A Z}{(A+h)^{2}}-\frac{g h Z}{(A+h)^{2}} \frac{\partial A}{\partial h}-\frac{g A}{A+h} \frac{\partial Z}{\partial h} \\
& \frac{d}{d t}\left(\frac{\partial Z}{\partial h}\right)=\frac{-b g A Z}{(A+h)^{2}}+\frac{b g h Z}{(A+h)^{2}} \frac{\partial A}{\partial h}+\left(\frac{b g A}{A+h}-m\right) \frac{\partial Z}{\partial h} \\
& \frac{d}{d t}\left(\frac{\partial A}{\partial b}\right)=r\left(1-\frac{2 A}{K}\right) \frac{\partial A}{\partial b}-\frac{g h Z}{(A+h)^{2}} \frac{\partial A}{\partial b}-\frac{g A}{A+h} \frac{\partial Z}{\partial b} \\
& \frac{d}{d t}\left(\frac{\partial Z}{\partial b}\right)=\frac{g A Z}{A+h}+\frac{b g h Z}{(A+h)^{2}} \frac{\partial A}{\partial b}+\left(\frac{b g A}{A+h}-m\right) \frac{\partial Z}{\partial b} \\
& \frac{d}{d t}\left(\frac{\partial A}{\partial m}\right)=r\left(1-\frac{2 A}{K}\right) \frac{\partial A}{\partial m}-\frac{g h Z}{(A+h)^{2}} \frac{\partial A}{\partial m}-\frac{g A}{A+h} \frac{\partial Z}{\partial m} \\
& \frac{d}{d t}\left(\frac{\partial Z}{\partial m}\right)=\frac{b g h Z}{(A+h)^{2}} \frac{\partial A}{\partial m}+\left(\frac{b g A}{A+h}-m\right) \frac{\partial Z}{\partial m}-Z \\
& \frac{d}{d t}\left(\frac{\partial A}{\partial a_{0}}\right)=r\left(1-\frac{2 A}{K}\right) \frac{\partial A}{\partial a_{0}}-\frac{g h Z}{(A+h)^{2}} \frac{\partial A}{\partial a_{0}}-\frac{g A}{A+h} \frac{\partial Z}{\partial a_{0}} \\
& \frac{d}{d t}\left(\frac{\partial Z}{\partial a_{0}}\right)=\frac{b g h Z}{(A+h)^{2}} \frac{\partial A}{\partial a_{0}}+\left(\frac{b g A}{A+h}-m\right) \frac{\partial Z}{\partial a_{0}} \\
& \frac{d}{d t}\left(\frac{\partial A}{\partial z_{0}}\right)=r\left(1-\frac{2 A}{K}\right) \frac{\partial A}{\partial z_{0}}-\frac{g h Z}{(A+h)^{2}} \frac{\partial A}{\partial z_{0}}-\frac{g A}{A+h} \frac{\partial Z}{\partial z_{0}} \\
& \frac{d}{d t}\left(\frac{\partial Z}{\partial z_{0}}\right)=\frac{b g h Z}{(A+h)^{2}} \frac{\partial A}{\partial z_{0}}+\left(\frac{b g A}{A+h}-m\right) \frac{\partial Z}{\partial z_{0}} \text {. }
\end{aligned}
$$

The initial conditions for Eq (2.22) are given by Eq (2.14). The set of equations was solved in GRIND. The results of this computation match closely with the results that were obtained using the GRIND 'timesens' command (Table 2.3). 



\section{Chapter 3}

\section{Detecting Tipping points in Ecological Models with Sensitivity Analysis}

G.A. ten Broeke, G.A.K. van Voorn, B.W. Kooi, J. Molenaar.

Mathematical Modelling of Natural Phenomena 11(4): pp. 47-72. 
Simulation models are commonly used to understand and predict the development of ecological systems, for instance to study the occurrence of tipping points and their possible ecological effects. Sensitivity analysis is a key tool in the study of model responses to changes in conditions. The applicability of available methodologies for sensitivity analysis can be problematic if tipping points are involved. In this paper we demonstrate that not considering these tipping points may result in misleading statistics on model behaviour. In turn, this limits the applicability of simulation models in ecological research. Tipping points are best revealed when asymptotic model behaviour is considered, i.e. by applying bifurcation analysis. Bifurcation analysis, however, is limited to deterministic dynamic models, whereas many ecological simulation models are nondeterministic and can only be analysed using sensitivity analysis methodologies. In this paper we explore the possibilities for applying methodologies of sensitivity analysis to analyse models with tipping points. The Bazykin-Berezovskaya model, a deterministic ecological model of which the structure regarding tipping points is known a priori, is used as case study. We conclude that important clues about the occurrence of tippings points can be revealed from different sensitivity analysis methodologies, if proper statistical and graphical measures are used. The results raise awareness about how tipping points affect temporal model responses in ecological simulation models, and may also be more generally applicable for nondeterministic models that cannot be analysed using bifurcation analysis.

\subsection{Introduction}

Ecological researchers and managers of natural systems commonly use simulation models to understand and predict the effects of drivers on ecological systems (Schmolke et al. 2010). Most ecological systems are Complex Adaptive Systems with many interacting, biotic components, and feedbacks (Schlueter et al. 2012). The applicability of simulation models is determined not only by the validity of these models (i.e. whether the models are a proper and useful simplified representation of the modelled system), but also by our ability to analyse them. Model analysis is vital during all steps in the development and use of ecological models (Jakeman et al. 2006, Schmolke et al. 2010), including model testing, calibration, validation, uncertainty estimation, and gaining a better understanding of model behaviour. Reflecting the complexity of the modelled system, many simulation models are also of considerable complexity. This generates a demand for the development and application of model analysis methodologies. Without the right methodologies a proper model analysis is not possible, and simulation models are of limited use.

We consider two important tools for model analysis, namely sensitivity analysis and bifurcation analysis. Sensitivity analysis quantifies the effects of changes in the parameters (including initial conditions) on the model output (Hamby 1994, Saltelli et al. 2004a, Cariboni et al. 2007, Campolongo et al. 2011). Its main use is to estimate which parameters are the most influential on certain model outputs. This information is useful for instance to quantify the information content of data for a given model, or to establish what parameters to focus on in validation experiments. Bifurcation analysis, on the other hand, considers changes in the topology of the phase 
space (i.e. qualitative changes) of the model with changes in parameters (including initial conditions). A lack of change in the topology with parameter changes may indicate resilience, i.e. the capacity of the modelled system to withstand pressures without undergoing drastic changes (Walker et al. 2004). Changes in topology may indicate tipping points that can lead to catastrophic shifts - fast, large-scale, and irreversible changes from one system state to another under relatively small changes in drivers (Folke et al. 2004, Scheffer and Carpenter 2003, Scheffer et al. 2009, Boettiger et al. 2013). Tipping points are either bifurcations or separatrices (Guckenheimer and Holmes 2013, Wiggins 1990, Kuznetsov 2004, Seydel 2010). A bifurcation is a specific parameter setting at which a qualitative change in model behaviour occurs. A separatrix is a manifold in state space that separates two domains of attraction of neighbouring attractors. In the latter case there are at least two alternative types of asymptotic model behaviour, and the initial conditions determine to which attractor the model will eventually evolve if no further manipulations of the system occur.

For many ecological management applications there is an interest in tipping points (Folke et al. 2004) and resilience, i.e. the asymptotic model behavioural features. For those applications bifurcation analysis would be the preferred method for analysing models. However, the application of bifurcation analysis is restricted by the complexity, as well as the type of many ecological models. Bifurcation analysis is well-suited for the analysis of deterministic dynamic models (DAEs or differential and algebraic equations) with a limited number of model variables and parameters, for which semiautomated tools are available, such as MATCONT (Dhooge et al. 2003) and AUTO07P (Doedel and Oldeman 2009). For complex models with a large number of parameters it is often not feasible to analyse the model that way. Furthermore, many types of models cannot at all be analysed using bifurcation analysis. The reason is that for differential equation (ODE) models, bifurcation analysis methods rely on the use of derivatives of the model equations, but these are not available for models with stochastic terms, or models where we have no explicit model equations available, such as agent-based models. Features like tipping points and resilience can nevertheless be expected in complex ecological simulation models. Therefore there is a dire need for methodologies that can analyse these models (Crooks et al. 2008, Filatova et al. 2013, Levin et al. 2013).

Methodologies of sensitivity analysis may be useful as an alternative for analysing more complex and nondeterministic ecological models. Sensitivity analysis methodologies are broadly categorised as being either local or global (Cariboni et al. 2007). Local methodologies quantify the sensitivity at a specific point in parameter space, whereas global quantify it across a range of parameter space. In addition, there are hybrid methodologies (Rakovec et al. 2014, Sobol' and Kucherenko 2009) that apply local methodologies at different points in parameter space to obtain a distribution of local sensitivity indices across a range of parameter space. Local methodologies linearise the output around the point at which they are applied to reveal detailed information limited to the neighbourhood of this point, omitting nonlocal interaction effects. This linearisation involves the determination of partial derivatives and thus meets the same limitation as bifurcation analysis. Global methodologies, on the other hand, reveal information that is valid over the full parameter range under investigation, by sampling from this range, and thus include nonlocal interaction effects. Model output is typically aggregated into statistical measures across the sample 
range, which results in the loss of more detailed information about model behaviour, including resilience and tipping points. The issue is demonstrated by Fig 3.1, which contains the bifurcation diagram of the one-dimensional Allee model,

$$
\frac{d Y}{d t}=Y(Y-\zeta)(\kappa-Y)-H Y
$$

where $Y$ is the population density, $\kappa$ the carrying capacity, $\zeta$ the Allee threshold, and $H$ a harvest rate constant. In the bifurcation diagram $\kappa=1, \zeta=0.2$, and $H$ is varied. For $H>0.16$ the population goes extinct for all initial conditions, whereas for $H<0.16$ there are two alternative attractors, namely a stable positive steady state and the stable zero state, each with their own domain of attraction. Global sensitivity analysis methodologies typically aggregate samples (output) from the two domains of attraction into the same statistics. As a result, the qualitative difference in model behaviour between the domains of attraction is obscured. The analysis may correctly show that $H$, on average, is an influential parameter, but it will not show that $H$ is crucial in determining whether the model evolves to a positive stable state or that a tipping point exists.

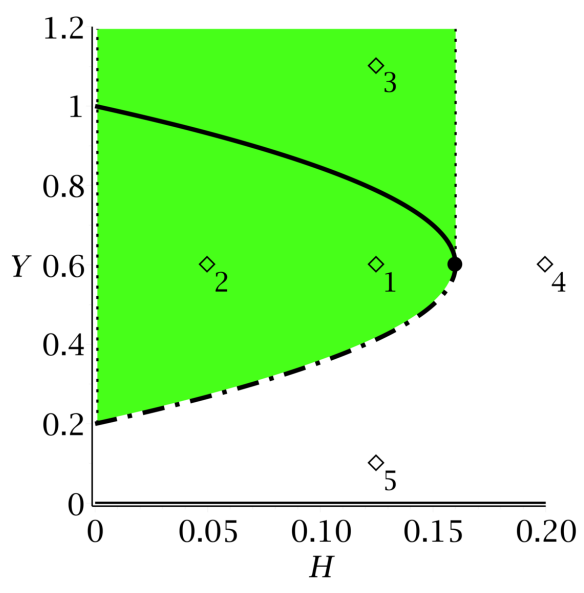

Figure 3.1: Bifurcation diagram of a model with tipping points Eq (3.1), with $\kappa=1$ and $\zeta=$ 0.2 . A bifurcation occurs at $H=0.16$. For $H<0.16$ there is bistability. Initial conditions $Y(0)$ in the green domain of attraction (samples 1, 2, and 3) evolve to the positive stable attractor indicated by the solid curve. Initial conditions in the white domain of attraction (samples 4 and 5) evolve to extinction. Statistics that are generated without distinction between these domains of attraction will be poor on information that is useful for tipping point analysis.

As it is, there are no standardised sensitivity analysis methodologies that are particularly suited to reveal or deal with tipping points. Ten Broeke, Van Voorn and Ligtenberg (2016) concluded that a one-factor-at-a-time approach is the best methodology for locating tipping points in ABMs. It is however a brute force method (i.e. based on performing many simulation runs), and hence it has obvious limitations in terms of computational costs. Because of the nondeterministic (i.e. stochastic) 
nature of many complex ecological models (including ABMs) many repetitive simulation runs have to be performed at different values of a single parameter before a distinction can be made between effects of tipping points and those of model stochasticity. This limits the application value of such sensitivity analysis methodologies for locating tipping points.

In this paper, we deal with the question of whether methodologies for sensitivity analysis can have a practical use for studying asymptotic features like tipping points and resilience in ecological models. By means of an example for which all the asymptotic features can be analysed using bifurcation analysis, we show how much information on tipping points and resilience can be obtained using methodologies for sensitivity analysis. The paper is organised as follows. In Section 3.2 three (groups of) methodologies for performing sensitivity analysis are discussed. In Section 3.3 the well-known Bazykin-Berezovskaya predator-prey model is introduced as a case study. The results of the analysis of the test case using sensitivity analysis methodologies are given in Section 3.4. Section 3.5 contains the discussion and conclusions.

\subsection{Sensitivity analysis methodologies}

In this section we provide a general overview of the sensitivity analysis methodologies that are applied in this paper. This section can be skipped without loss of readability by readers who have extensive knowledge of methodologies for sensitivity analysis, or who wish to skip the technical details.

\subsubsection{Local sensitivity analysis}

Local parameter sensitivities are typically expressed as the partial derivative of the model output with respect to a selected parameter (including initial conditions)

$$
s_{i, j}(t)=\frac{\partial Y_{j}(t)}{\partial \theta_{i}},
$$

where $t$ is time (i.e. these sensitivities may vary over time), $Y_{j}$ are the output variables, $\theta_{i}$ the parameters (including initial conditions), $i=1,2, \ldots, N_{p}$ with $N_{p}$ the number of parameters, and $j=1,2, \ldots, N_{v}$ with $N_{v}$ the number of variables or model outputs (see Table 3.1 for an overview of the used symbols). Eq (3.2) may be estimated for ODE models using the direct differential method (Dickinson and Gelinas 1976) (Appendix 3.A). Sensitivity indices are expressed in this method as additional differential equations to be solved alongside the original ODE model. Alternatively, finite differences methods can be used to estimate Eq (3.2) based on e.g. the difference between a model run in the nominal point and a model run with a slightly different value for the parameter for which the sensitivity is estimated.

In a one-at-a-time sensitivity analysis the local partial derivatives of model outputs with respect to any number of (preferably all) parameters are determined around a single point. Local sensitivity indices Eq (3.2) cannot be directly compared because parameters may have different units. Therefore, in addition to local sensitivity indices one usually reports elasticities, which have been normalised using the nominal parameter values 
Table 3.1: List of all general symbols used in the paper.

\begin{tabular}{|c|c|}
\hline Symbol & Meaning \\
\hline $\mathbf{A}$ & Sampling matrix (used only in Appendix) \\
\hline $\mathbf{A}_{B}$ & Recombinant sampling matrix (used only in Appendix) \\
\hline B & Alternative sampling matrix (used only in Appendix) \\
\hline $\mathbf{B}_{A}$ & Recombinant sampling matrix (used only in Appendix) \\
\hline$E(\cdot)$ & Expected value \\
\hline$e_{i, j}$ & Elasticity \\
\hline$f$ & Function; model output \\
\hline$G$ & Curve corresponding to global bifurcation \\
\hline$g(Y, \theta, t)$ & Vector field of ODE model (used only in Appendix) \\
\hline$H$ & Curve corresponding to Hopf bifurcation \\
\hline$i$ & Index for model parameters \\
\hline $\mathbf{J}$ & Jacobian matrix \\
\hline$I^{N_{p}}$ & $N_{p}$-dimensional unit hypercube (used only in Appendix) \\
\hline$j$ & Index for model outputs \\
\hline$l$ & Index for model parameters \\
\hline$m$ & Index for model outputs \\
\hline$N_{p}$ & Total number of parameters \\
\hline$N_{s}$ & Sample size in sampling method by Saltelli et al. (2010) \\
\hline$N_{v}$ & Total number of outputs \\
\hline$n$ & Number of samples per parameter in the factorial design \\
\hline$P$ & Probability density function (used only in Appendix) \\
\hline$S_{i, j}$ & Global first order variance-based sensitivity \\
\hline$\tilde{S}_{i, j}$ & Global total effect variance-based sensitivity \\
\hline$s_{i, j}$ & Local sensitivity \\
\hline$\tilde{s}_{i, j}$ & DELSA local sensitivity \\
\hline$t$ & Time \\
\hline$U(\cdot)$ & Uniform probability density \\
\hline$V(\cdot)$ & Variance \\
\hline$V_{i}$ & Partial variance attributed to parameter $i$ \\
\hline$W^{s}$ & Stable manifold in state space \\
\hline$W^{u}$ & Unstable manifold in state space \\
\hline$Y$ & Model output vector with elements $Y_{j}$ \\
\hline$\Theta$ & All parameters $\theta_{i}$ \\
\hline$\theta_{i}$ & Model parameter or initial condition \\
\hline
\end{tabular}




$$
e_{i, j}(t)=\frac{\theta_{i}}{Y_{j}(t)} \frac{\partial Y_{j}(t)}{\partial \theta_{i}} .
$$

Elasticities have a straightforward interpretation. For example, an elasticity of 5 indicates that a $1 \%$ change in the parameter causes a $5 \%$ change in the output with respect to the nominal point. Elasticities are not well-defined when the output variable $Y_{j}$ goes to zero. For negative parameter values, a positive elasticity would indicate that a positive parameter change causes a negative change in the output. It is therefore common to report the absolute value of the elasticity, which expresses the magnitude of the output change that is caused by a parameter change.

Local sensitivity analysis is a useful methodology for assessing which parameters in ecological models are the most influential around a nominal point. It has the added advantage of being computationally cheap. Since local sensitivity analysis involves linearisation of the output response around the nominal point, it omits nonlocal interaction effects. Some care should be taken when applying local sensitivity analysis to models with tipping points, because at a tipping point there is a discontinuity in the output and the partial derivatives are thus not well-defined.

Besides local sensitivity analysis methodologies based on partial derivatives, one can also perform one-factor-at-a-time (OFAT) sensitivity analysis. Similar to other local sensitivity analysis methodologies, OFAT considers only changes in a single parameter with respect to a nominal point, while keeping all other parameters constant. Instead of linearising the output around the nominal point, in OFAT the parameter is varied stepwise over a larger range. The model output is plotted as a function of the varied parameter. OFAT is not directly aimed at quantifying sensitivities, but is useful for revealing qualitative relations between individual parameters and the model output. OFAT can reveal whether the output changes linearly or non-linearly as a function of changes in a single model parameter, and thus can help to reveal whether tipping points are crossed as the parameter is changed (Ten Broeke, Van Voorn and Ligtenberg 2016).

\subsubsection{Global sensitivity analysis}

Global sensitivity analysis considers parameter changes over a larger range of parameter space and may thus include nonlocal interaction effects. The range is defined by assigning uniform probability density functions to the parameters that are included in the analysis. The variation in the output variables over the range is then attributed to the variations in the different parameters. The Sobol' method (Sobol' 2001) is one of the most commonly used methodologies for global sensitivity analysis. This method is based on a decomposition of the output variance under the assumption that all parameters are independent (Jansen 1999, Saltelli et al. 2010)

$$
V\left(Y_{j}(t)\right)=\sum_{i} V_{i}(t)+\sum_{l>i} V_{i, l}(t)+\ldots+V_{1,2, \ldots, N_{p}}(t)
$$

where $V\left(Y_{j}(t)\right)$ is the total output variance over the considered region of parameter space at time $t, V_{i}(t)$ is the part of the variance that is attributed to the parameter $\theta_{i}$, 
and $V_{i, l}(t)$ is the part that is attributed to the interaction between $\theta_{i}$ and $\theta_{l}$. Higher order terms represent higher order interaction effects. Sensitivity indices are defined by normalising the terms of Eq (3.4) through division by the total variance. The most commonly reported sensitivity indices are the first-order index and the totalorder index (Saltelli et al. 2008). The first-order index estimates the variance that is explained by a single parameter excluding interaction effects,

$$
S_{i, j}(t)=\frac{V_{i}(t)}{V\left(Y_{j}(t)\right)} .
$$

The total-order sensitivity includes all the interaction effects with other parameters,

$$
\tilde{S}_{i, j}(t)=\frac{1}{V\left(Y_{j}(t)\right)}\left(V_{i}(t)+\sum_{l} V_{i, l}(t)+\ldots+V_{1,2, \ldots, N_{p}}(t)\right) .
$$

First- and total-order indices always attain values between 0 and 1 . However, the sum of the total-order indices exceeds 1 if the model has interaction effects. A large difference between $\mathrm{Eq}$ (3.5) and Eq (3.6) indicates that interaction effects are influential.

The first-order sensitivity Eq (3.5) can be expressed in terms of conditional variances and expectations of the model output (Sobol' 2001) (Appendix 3.D)

$$
S_{i, j}(t)=\frac{V_{\theta_{i}}\left(E_{\theta_{\sim i}}\left(Y_{j}(t) \mid \theta_{i}\right)\right)}{V\left(Y_{j}(t)\right)}
$$

where $E(\cdot)$ is the expectation value, $V(\cdot)$ the variance (see also Table 3.1 ), and indicates 'all except', i.e. all parameters are varied except parameter $\theta_{i}$.

To evaluate Eq (3.7), we first compute the expectation value of the model output over all other parameters, keeping $\theta_{i}$ fixed. We then compute the variance of the resulting expectation value over the possible values of $\theta_{i}$. Similarly, the total-order sensitivity indices may be expressed as

$$
\tilde{S}_{i, j}(t)=\frac{E_{\theta_{\sim i}}\left(V_{\theta_{i}}\left(Y_{j}(t) \mid \theta_{\sim i}\right)\right)}{V\left(Y_{j}(t)\right)}=1-\frac{V_{\theta_{\sim i}}\left(E_{\theta_{i}}\left(Y_{j}(t) \mid \theta_{\sim i}\right)\right)}{V\left(Y_{j}(t)\right)}
$$

where the law of total variance is used to obtain the rightmost expression.

If for ODE models explicit expressions for the steady states are available, Eqs (3.73.8) are evaluated analytically by inserting the steady state values to which the model evolves given the choice of parameters and initial conditions, and performing integrations over the parameters to calculate the means and variances. If explicit expressions are not available, a number of methodologies are available to estimate Eqs (3.7-3.8) based on samples from the parameter space (i.e. brute force approach) (Saltelli et al. 2010). The most direct sampling method is a factorial design, in which the considered parameter space is divided into (possibly equidistant) steps, obtaining a chessboardlike grid. The intersection points of this grid are used as sample points. A grid 
of five points for four parameters thus gives $5^{4}=625$ possible parameter combinations and the same number of model simulations (excluding replicates to account for stochasticity).

The factorial design provides a straightforward way to evaluate Eqs (3.7-3.8), but since the number of sample points increases exponentially with the number of parameters, it is impractical for models with a large number of parameters. For models with many parameters, more cost efficient sampling methodologies have been proposed. One such method is aimed at the estimation of the partial variances based on covariances (Saltelli et al. 2010) (explained in detail in Appendix 3.F). This method has limitations in that it can yield negative estimates for the partial variances. In this paper we will use both the factorial design and the method by Saltelli et al. (2010) to estimate global sensitivities. Note that the Sobol' method in general is not applicable if the parameters are dependent, because the variance decomposition Eq (3.4) holds only for independent parameters.

\subsubsection{Hybrid sensitivity analysis}

Hybrid methodologies of sensitivity analysis compute local sensitivity indices at various points in parameter space, thus combining local and global sensitivity analysis. The local sensitivity index in each point measures the local sensitivity around that point. The distribution of sensitivity indices across a range of parameter space measures the global sensitivity over the region. The DELSA methodology (Rakovec et al. 2014) converts local sensitivity indices into variance-based sensitivity indices, enabling direct comparison with the Sobol' methodology. Computing the variance of a Taylor expansion of the model output around the point where the local sensitivity index is evaluated yields, (Rakovec et al. 2014, Mood et al. 1974)

$$
V\left(Y_{j}(t)\right) \approx \sum_{i=1}^{N_{p}}\left|\frac{\partial Y_{j}(t)}{\partial \theta_{i}}\right|_{\Theta}^{2} V\left(\theta_{i}\right)
$$

where the summation runs over all parameters, $\Theta=\left(\theta_{1}, \theta_{2}, \cdots, \theta_{N_{p}}\right) \in \mathbb{R}^{N_{p}}$ denotes the point in parameter space where the local sensitivity index is evaluated. $V\left(\theta_{i}\right)$ is the variance of the parameters around this point, given the (uniform) probability distribution that is assigned to the parameters. Each term in the sum denotes the output variance that is attributed to the corresponding parameter. Sensitivity indices are obtained by normalising these terms with respect to the total output variance around the points

$$
\tilde{s}_{i, j}=\frac{1}{V\left(Y_{j}(t)\right)}\left|\frac{\partial Y_{j}(t)}{\partial \theta_{i}}\right|_{\Theta}^{2} V\left(\theta_{i}\right), \quad i=1,2, \cdots, N_{p} .
$$

Note that this sensitivity index $\tilde{s}_{i, j}$ is a local sensitivity measure that estimates the sensitivity around the point where Eq (3.10) is evaluated. Information on global sensitivities is obtained by considering the probability density of $\mathrm{Eq}$ (3.10) across a range of parameter space. The DELSA methodology has the advantage of measuring not only global sensitivities over such a range, but also giving more detailed results 
for points within this range. For example, a parameter may be shown to be influential in certain parts of parameter space, but not in other parts. Unlike the Sobol' method, which is properly normalised only for independent parameters, DELSA does not assume that the model parameters are independent. In addition, the computational costs have been reported to be lower than the costs of the Sobol' method (Rakovec et al. 2014), but nevertheless are still much higher than the costs of local sensitivity analysis.

\subsection{Case description}

\subsubsection{Model}

Our case study involves the analysis of the Bazykin-Berezovskaya predator-prey model with an Allee effect for the prey species (Bazykin 1998). The Allee effect refers to the observation that many populations of species do not only suffer from detrimental effects when densities are high, i.e. because of intraspecific competition, but also when densities are low (Allee 1931, Kramer et al. 2009). This may result, for instance, from difficulties in finding mates or cooperative feeding (Kramer et al. 2009), or a positive relationship between a component of individual fitness and the number of conspecifics (Stephens et al. 1999). A distinction is commonly made between weak and strong Allee effects (Taylor and Hastings 2005). A weak Allee effect indicates there are negative effects but not such that the population will go extinct. A strong Allee effect, as occurs in the Bazykin-Berezovskaya model, indicates there is a certain threshold density below which the population will go extinct.

The Bazykin-Berezovskaya model is well-known and mathematically tractable, and has been extensively analysed. The model is well-suited to demonstrate the issues we address in this paper, because all relevant types of tipping points occur in the model, namely separatrices (and hence alternative attractors), local bifurcations, and also global bifurcations that involve the (dis)appearance of separatrices. This makes it an ideal test case to study what information about tipping points can be found based on the application of methodologies for sensitivity analysis.

The nondimensional Bazykin-Berezovskaya model reads

$$
\begin{aligned}
\frac{d Y_{1}}{d t} & =Y_{1}\left(Y_{1}-\zeta\right)\left(\kappa-Y_{1}\right)-Y_{1} Y_{2}, \\
\frac{d Y_{2}}{d t} & =\gamma\left(Y_{1}-h\right) Y_{2},
\end{aligned}
$$

where $Y_{1}$ is the prey density, $Y_{2}$ the predator density, $\kappa$ the carrying capacity (the positive monoculture steady state density of the prey species), $\gamma$ the conversion factor from prey to predator, and $h$ the predator mortality rate, scaled to the conversion factor $\gamma$. Parameter $\zeta$ represents the Allee threshold. A concise overview of the model variables and parameters is given in Table 3.2.

\subsubsection{Bifurcation analysis}

The Bazykin-Berezovskaya model has been analysed thoroughly in the literature. In particular numerical techniques based on defining boundary value problems have been 
Table 3.2: List of the variables, parameters and initial conditions used in the Allee model. Also indicated are the nominal values (if applicable) and whether they are fixed or not in the sensitivity analysis.

\begin{tabular}{llll}
\hline Symbol & $\begin{array}{l}\text { Nominal } \\
\text { value }\end{array}$ & Fixed/Free & Meaning \\
\hline$Y_{1}$ & - & - & Prey population density \\
$Y_{2}$ & - & - & Predator population density \\
\hline$Y_{1}(0)$ & 0.9 & Fixed & Initial condition of $Y_{1}$ \\
$Y_{2}(0)$ & 0.1 & Free & Initial condition of $Y_{2}$ \\
$\gamma$ & 1. & Fixed & Conversion factor \\
$\kappa$ & 1. & Fixed & Prey carrying capacity \\
$h$ & 0.9 & Free & Predator mortality rate \\
$\zeta$ & 0.5 & Free & Allee threshold (prey) density \\
\hline$H$ & - & - & Prey harvesting rate (Eq 3.1) \\
\hline
\end{tabular}

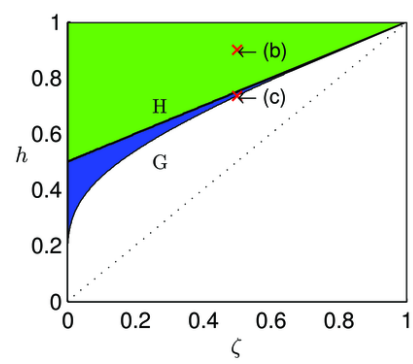

(a)

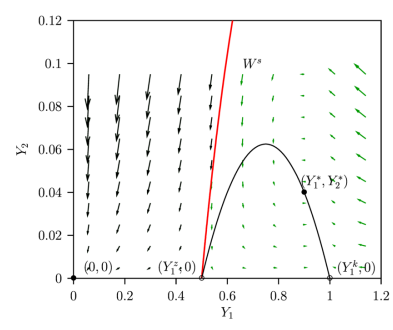

(b)

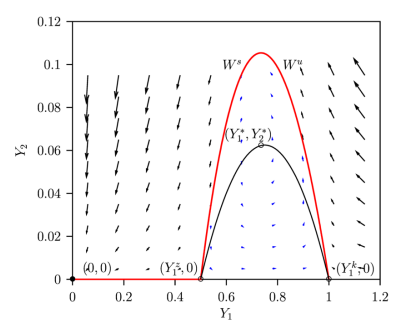

(c)

Figure 3.2: a: Two-parameter bifurcation diagram of the Bazykin-Berezovskaya model for $\zeta$ and $h$, with other parameters at their nominal values. $H$ indicates the Hopf bifurcation curve. $G$ indicates the global bifurcation curve. In the green region there are two alternative attractors, namely $Y^{*}(\mathrm{Eq} 3.12)$ and $Y^{0}=[0,0]$. In the blue region the nontrivial positive steady state has turned into a periodic attractor. In the white region the community always goes extinct. The dotted line indicates a transcritical bifurcation curve. b: The vector field for $Y_{1}$ and $Y_{2}$ with all parameters in the nominal point (marked as (b) in Fig 3.2a) shows that there is bistability. The black line is the null-cline where the time-derivative of $Y_{1}$ is zero. The red line $W^{s}$ is the stable manifold that terminates at $\left(Y_{1}^{z}, 0\right)$ and acts as a separatrix. c: At $h \approx 0.735442$ and all other parameters at their nominal value (marked as (c) in Fig 3.2a), the stable manifold $W^{s}$ and the unstable manifold $W^{u}$ connect the steady states $\left(Y_{1}^{z}, 0\right)$ and $\left(Y_{1}^{k}, 0\right)$ and act as a separatrix. Inside the manifold, the solution is a stable limit cycle, whereas outside the manifold the solution goes to extinction. 
used to localize a heteroclinic point-to-point connection (Van Voorn et al. 2007). This connection is not structurally stable, and has a biological interpretation as overexploitation. We summarise these results for convenience before proceeding to the sensitivity analysis.

$\operatorname{Eq}(3.11)$ has four steady state solutions, three trivial ones $\left(Y^{0}=[0,0], Y^{z}=[\zeta, 0]\right.$, $\left.Y^{k}=[\kappa, 0]\right)$, and a nontrivial one

$$
Y^{*}=\left[h, h \zeta-\kappa \zeta-h^{2}+h \kappa\right]
$$

The Jacobian matrix for the nontrivial steady state is

$$
\left.\mathbf{J}\right|_{Y=Y^{*}}=\left(\begin{array}{cc}
h(\zeta+\kappa-2 h) & -h \\
\gamma(h-\kappa)(\zeta-h) & 0
\end{array}\right)
$$

The determinant of $\mathbf{J}$ is used to determine the transcritical bifurcation of the nontrivial steady state, i.e. the point where the predator species can enter the system and sustain itself. The trace is used to determine the Hopf bifurcation, i.e. the point where the nontrivial stable steady state becomes unstable and periodic behaviour appears, giving rise to (in this case stable) limit cycles.

The bifurcation diagram displayed in Fig 3.2a shows the asymptotic behaviour of the model as function of $\zeta$ and $h(\gamma=1, \kappa=1)$. Three transcritical bifurcations are found at $h^{0}=0, h^{z}=\zeta$, and $h^{k}=\kappa$, and the Hopf bifurcation occurs at

$$
h^{H}=\frac{1}{2}(\kappa+\zeta)
$$

which is indicated by the curve $H$ in Fig 3.2a. The model displays bistability for a significant part of parameter space. When the nontrivial steady state is positive, there exists a unique manifold that terminates at the trivial steady state $Y^{z}$ and acts as a separatrix (Fig 3.2b). Initial conditions on the right hand side of the separatrix converge to $Y^{*}$ and on the left hand side to the trivial steady state $Y^{0}$. Thus, in the green parameter region in Fig 3.2a the model may converge to the nontrivial steady state $Y^{*}$, or to the trivial steady state $Y^{0}$, depending on the initial conditions. In the blue parameter region the model may converge either to the limit cycle around $Y^{*}$, or to $Y^{0}$.

The heteroclinic point-to-point connection links the saddle steady state $Y^{k}$ to the saddle steady state $Y^{z}$, as the stable manifold $W_{s}$ belonging to $Y^{z}$ overlaps with the unstable manifold $W_{u}$ belonging to $Y^{k}$ (see Fig 3.2c for the connection at the parameter set $\zeta=0.5, h^{G} \approx 0.735442$ ). The two-parameter continuation of this curve, indicated by $G$ in Fig 3.2a, is obtained by using AUTO(Doedel and Oldeman 2009 ) with a set of boundary conditions (Van Voorn et al. 2007, 2010). For values of $h>h^{G}$ (green and blue parameter regions) the system displays bistability. For values $h<h^{G}$ the separatrix has disappeared, and $Y^{0}$ is the sole attractor (white parameter region). 


\subsection{Results of sensitivity analysis}

In this section we apply the sensitivity analysis methodologies that are presented in Section 3.2 to the Bazykin-Berezovskaya model and discuss the obtained results. We consider only the predator density $Y_{2}$ as model output and parameters $h$ and $\zeta$ as inputs to demonstrate the principles. The parameters $\gamma$ and $\kappa$ are fixed at the nominal values, and $Y_{1}(0)$ is fixed such that its value lies between $\kappa=1$ and the highest sampled value of $\zeta$. Note, that for the sensitivity analysis we assume a researcher who is aware that tipping points may exist, but who is not able for whatever reason to perform bifurcation analysis. The aim is to evaluate which of the sensitivity analysis methodologies can be useful in detecting, or revealing clues about, tipping points such as the transcritical bifurcation, the Hopf bifurcation, the separatrix and the heteroclinic connection.

\subsubsection{Results of local sensitivity analysis}

A classical starting point of a sensitivity analysis is to quantify the local sensitivities Eq (3.2) as given in subsection 3.2.1. For finite values of $t$ we use the direct differential method (Appendix 3.A) to determine these local sensitivities (details of the calculation are given in Appendix 3.B). The results show that the predator density initially is most sensitive to the initial condition $Y_{2}(0)$, which is an obvious result (Fig 3.3). As the simulation proceeds, the sensitivity to $Y_{2}(0)$ decreases to zero, while the sensitivity to $h$ becomes the largest. The elasticities show the same outcome: the sensitivity of the model for $Y_{2}(0)$ decreases to zero, while the sensitivity for $h$ becomes the largest.

For long simulation times (effectively $t \rightarrow \infty$ ) the model and its parameter sensitivities evolve to steady state values. The steady state sensitivities can also be determined analytically by taking the derivative in the positive steady state $Y^{*} \mathrm{Eq}(3.12)$,

$$
\begin{gathered}
s_{\zeta}^{*}=\frac{\partial Y_{2}^{*}}{\partial \zeta}=h-\kappa, \\
s_{h}^{*}=\frac{\partial Y_{2}^{*}}{\partial h}=\kappa+\zeta-2 h,
\end{gathered}
$$

and

$$
s_{Y_{2}(0)}^{*}=0
$$

where the index $j$ in Eq (3.2) is dropped from the notation, and for the index $i$ we write $\zeta, h$, or $Y_{2}(0)$. Table 3.3 gives these steady state sensitivities, along with the associated elasticities Eq (3.3). The outcomes show a close match with those in Fig 3.3 for sufficiently large values of $t$.

Note that the above results estimate the effects of small parameter changes around the nominal point. For larger parameter changes with respect to the nominal set, tipping points and other nonlinearities may become important, in which case these results are no longer valid. For example, whereas $s_{Y_{2}(0)}=0$ in the nominal setting, Fig 


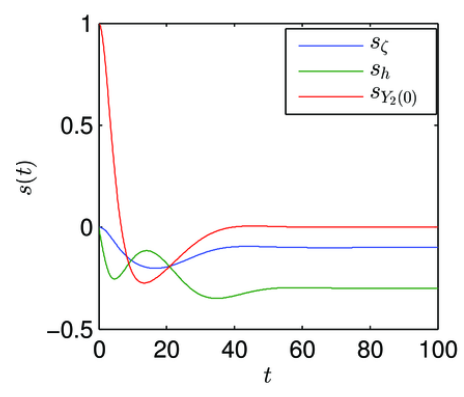

(a)

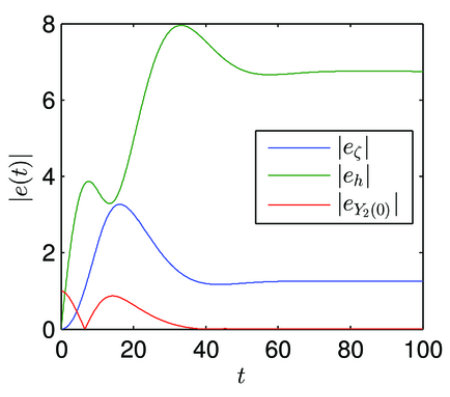

(b)

Figure 3.3: a : The local sensitivities of $Y_{2}(t)$ of the Bazykin-Berezovskaya model with respect to parameters $\zeta$ (in blue), $h$ (in green), and the initial condition $Y_{2}(0)$ (in red). Other parameters are set at their nominal values. Over time the sensitivity to $Y_{2}(0)$ decreases to around zero, while that of $h$ seems to be the largest. b: The respective elasticities, i.e. dimensionless sensitivities. Also in this case the sensitivity to $h$ is found to be the largest.

$3.2 \mathrm{~b}$ shows that for larger parameter changes the value of $Y_{2}(0)$ determines whether the model evolves to the positive steady state, or to extinction. Thus, anywhere away from the separatrix $W^{s}$ the sensitivity $s_{Y_{2}(0)}$ asymptotically goes to zero, but at the separatrix the sensitivity is not well-defined.

Table 3.3: Local and global sensitivities in the steady state $\left(Y_{1}^{*}, Y_{2}^{*}\right)$ for the output variable $Y_{2}$. The local sensitivities and elasticities are computed at the nominal point (Table 3.2). The first-and total-order global sensitivities Eqs (3.7-3.8) are computed over the stable steady state region (green) in Fig 3.2a, assuming that the initial conditions are such that the model converges to the positive steady state $Y^{*}$. The computation of these global sensitivity indices is given in Appendix 3.E. We have dropped the index $j$ in Eq 3.2 from the notation.

\begin{tabular}{llll}
\hline & $\zeta$ & $h$ & Description \\
\hline$s_{i}^{*}$ & -0.1 & -0.3 & Local sensitivity Eq $(3.2)$ in nominal point \\
$\left|e_{i}^{*}\right|$ & 1.25 & 6.75 & Local elasticity Eq $(3.3)$ in nominal point \\
\hline$S_{i}^{*}$ & .593 & 1.25 & First-order global sensitivity Eq $(3.7)$ \\
$\tilde{S}_{i}^{*}$ & 0.267 & 0.167 & Total-order global sensitivity Eq $(3.8)$
\end{tabular}

To explore how the output responds to larger changes of individual model parameters, we perform an OFAT sensitivity analysis. The results reveal tipping points where the output goes to zero for all three parameters (Fig 3.4). For $h$ and $\zeta$ this indicates the presence of a transcritical bifurcation, and for $Y_{2}(0)$ it indicates the presence of a separatrix. This shows that OFAT can function as an appropriate starting point for detecting tipping points when methods of bifurcation analysis are not available. Note, however, that OFAT considers only changes in individual parameters and does not scan the full parameter space. As a result, tipping points in other parts of parameter space will not be detected. 


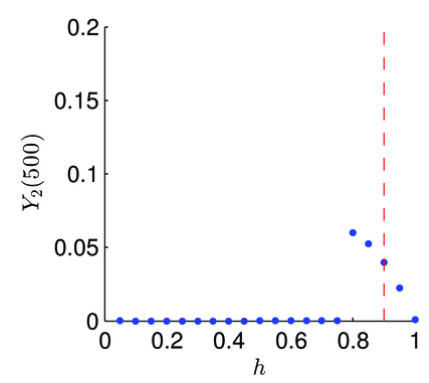

(a)

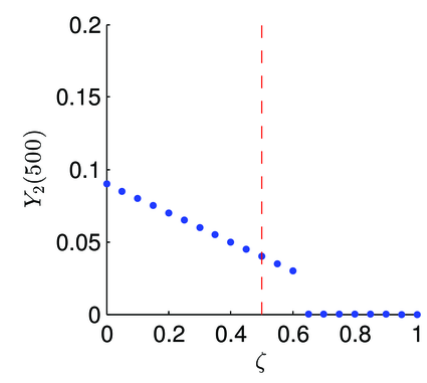

(b)

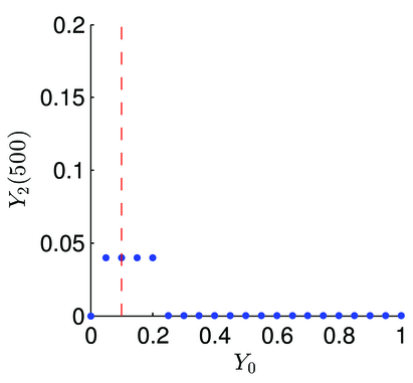

(c)

Figure 3.4: In the OFAT analysis each parameter was varied individually, starting from the nominal parameter and initial condition settings. The output is plotted as a function of the parameter, and the nominal parameter value is indicated by the red dashed line. The results at $t=500$ clearly reveal the presence of tipping points. a: For small values of $h, Y_{2}$ converges to zero, until a tipping point is crossed where the output converges to the positive steady state. b: For small values of $\zeta$ the model converges to the positive steady state. As $\zeta$ increases, a tipping point is crossed after which the population goes extinct. c: For low values of $Y_{2}(0)$ the output converges to the positive steady state. Changes in $Y_{2}(0)$ do not change the value of this steady state, but as $Y_{2}(0)$ increases a separatrix is crossed after which the output goes to zero.

\subsubsection{Results of global sensitivity analysis}

Here we apply the two methodologies to determine variance-based sensitivities Eqs (3.7-3.8) that were introduced in Section 3.2.2. The sampling of the first methodology is based on a factorial design, with $n=10,20$, and 40 equidistant values for each parameter under investigation $\left(\zeta, h\right.$ and $\left.Y_{2}(0)\right)$, giving $10^{3}=1000,20^{3}=8000$ and $40^{3}=64,000$ parameter combinations, respectively. The sampling of the second methodology by Saltelli et al. (2010) is based on Monte Carlo sampling, with base sample size $N_{s}=500,1000$ and 2000, giving 4000, 8000, and 16,000 parameter combinations, respectively. As both methodologies use brute force simulation, the computational costs of both methodologies can be considered to be proportional to the number of investigated parameter combinations. For both methodologies, $h$ and $\zeta$ were varied between 0 and 1 , and $Y_{2}(0)$ between 0 and 0.2 .

The resulting first-order Eq (3.5) and total-order Eq (3.8) global sensitivities for $Y_{2}(t=10), Y_{2}(t=50)$, and $Y_{2}(t=200)$ are given in Table 3.4 in Appendix 3.E, for time points $t=10, t=50$, and $t=200$, respectively. Fig 3.5 shows the time evolution of the first-order and total-order sensitivities determined by using the factorial design. The sensitivities show no long-term changes after $t=100$. The fluctuations after $t=100$ are most likely caused by the fact that in some runs the system evolves to a limit cycle. The most influential parameter on shorter simulation times is $h$, whereas $\zeta$ is more influential for longer simulation times. Note that this latter result contrasts with the results of the local sensitivity analysis, which indicate that $h$ is the most influential parameter for long simulation times. We will return to this finding in Section 3.4.3. The large differences between the first-order and total-order sensitivities indicate that interaction effects are important. 


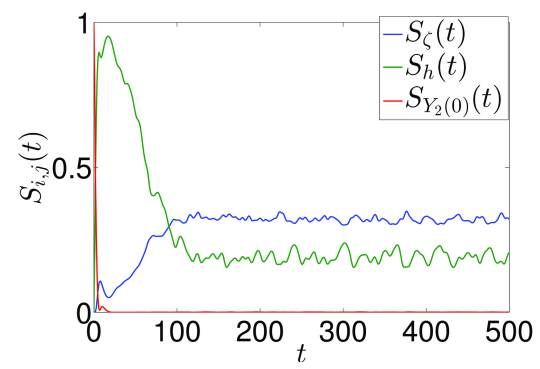

(a)

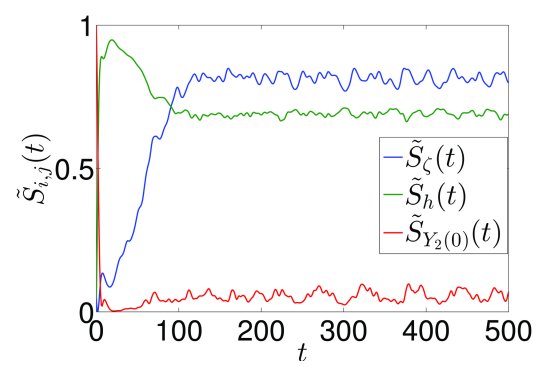

(b)

Figure 3.5: First-order (a) and total-order (b) sensitivity indices as a function of time. On shorter time-scales $h$ is the most influential parameter, whereas on longer timescales $\zeta$ is more influential. The difference between the first- and total-order indices shows that interaction effects are important. A large portion of the variance is attributed to the interaction between $h$ and $\zeta$.

Overall there is a rather good match between the results of the factorial design and the method by Saltelli et al. (2010) for $t=10$ and $t=50$. For $t=200$, however, the outcomes differ considerably. The factorial design indicates that $\zeta$ is the most influential parameter, whereas the method by Saltelli et al. (2010) indicates that $h$ is the most influential. The difference between the methodologies is caused by a small number of sample points with values of $h$ close to zero. The convergence of $Y_{2}$ to the steady state value $Y^{0}$ requires a very long simulation time for small values of $h$. In the factorial design, the lowest value of $h$ in the sample grid is sufficiently large for the sample runs to have converged around $t=200$. In the method by Saltelli et al. (2010), however, the sampling is random and very small values of $h$ can thus occur, at which convergence has not yet occurred around $t=200$. This explanation was checked by performing some extended simulations for these small values of $h$. After inclusion of the results of these extended simulations the method by Saltelli et al. (2010) also indicates $\zeta$ to be the most influential parameter.

The aggregated statistics presented by Eq (3.5) and Eq (3.8) are not informative in terms of the detection of possible tipping points. As an alternative, non-aggregated statistics may be used, preferably in a graphical representation. Fig 3.6 displays the results obtained using the factorial design for $t=50$ (left panels) and $t=500$ (right panels). Samples from the three parameter regions with qualitatively different behaviour are indicated by colour and shape, where green dots indicate samples from the region with bistability where $Y^{*}$ is stable, blue diamonds are from the region with bistability where limit cycles occur, and black crosses are from the region in which there is always extinction (see also Fig 3.6a). The values of $Y_{2}(t)$ are aggregated according to the parameter that is investigated while all other parameters are 'free'. For example in Fig 3.6c all samples are clustered per value of $\zeta$, while the other parameter values (for $h$ and $Y_{2}(0)$ ) are considered to be unknown. We refer to this as all-but-one-simultaneously (ABOS) as compared to one-factor-at-a-time (OFAT) sensitivity analysis. The red squares indicate the mean values of $Y_{2}(t)$ of the samples clustered per value of the investigated parameter. The variance of these means is the estimation of the first-order sensitivity index Eq (3.7). Since we are interested in 
revealing tipping points where the model evolves to extinction, we plot the minimum value of the limit cycle for samples that show limit cycles.

Based on the graphical ABOS results in Fig 3.6c parameter $\zeta$ can be considered to be the most influential at $t=50$. The decreasing mean (i.e. red squares) with increasing value of $\zeta$ corresponds to the decreasing green parameter region on which bistability occurs (compare to Fig 3.2a and Fig 3.6a). The cloud of black crosses is due to the fact that at $t=50$ the model still displays considerable transient behaviour. For $t=500$ the transient behaviour has disappeared and the means are near zero for $\zeta>$ 0.5 (Fig 3.6d), while also the diversity in values of $Y_{2}(t)$ decreases for increasing values of $\zeta$. It can be concluded from this figure that the region of bistability decreases for increasing values of $\zeta$, i.e. $\zeta$ is an influential parameter in determining the bistability in the model.

In Fig 3.6e the ABOS of parameter $h$ at $t=50$ is displayed. The samples from the green parameter region are limited to $h>0.5$, and furthermore the nonzero values of $Y_{2}(t)$ increase for decreasing values of $h$. This is in accordance with the bifurcation diagram in Fig 3.6a. Starting from the right, the means increase then decrease, until around $h=0.5$ the means become zero. This is a strong indication of a tipping point around this value of $h$. Indeed, in Fig 3.6b the one-parameter continuation curve of $Y^{*}$ as function of $h$ is displayed (obtained using AUTO (Doedel and Oldeman 2009)), where $\zeta=0.5$. Within the range $0.75<h<1$ the positive steady state is stable, while within $0.5<h<0.75$ it is unstable. After the destabilization at the Hopf bifurcation at $h=0.75$ the heteroclinic bifurcation occurs at $h \approx 0.735442$, and then the transcritical bifurcation at $h=0.5$. The means in Fig 3.6 f seem to follow the one-parameter continuation curve of $Y^{*}$ rather faithfully. In addition, nearing the transcritical bifurcation there is a marked increase in variance. The nonzero transient behaviour is limited to very low values of $h$. This is explained by the long simulation time that is required for the system to evolve to $Y^{0}$ for small values of $h$. When $t=500$ the increase in variance is less pronounced, while the 'spike' of black crosses has disappeared. One may suspect the existence of bistability based on the existence of two separate clouds (namely the green and the blue ones) and the divergence between the green cloud and the red means for decreasing $h$. Although for $0.5<h<0.75$ the values of the positive samples increase with decreasing $h$, the means decrease because a larger number of samples converges to extinction.

The sensitivity of the model to the initial condition $Y_{2}(0)$ is shown in Fig $3.6 \mathrm{~g}$ at $t=50$ and Fig $3.6 \mathrm{~h}$ at $t=500$. From both figures it can be concluded that $Y_{2}(0)$ does not contribute considerably to the model output. While for $t=50$ there is still a cloud of black crosses, at $t=500$ this has disappeared. The means however do not vary considerably as function of $Y_{2}(0)$, which corresponds to the earlier findings that $Y_{2}(0)$ is not an influential parameter.

\subsubsection{Results of hybrid sensitivity analysis}

The DELSA methodology combines aspects of local and global sensitivity analysis and is aimed at obtaining more detailed information on the distribution of the sensitivity indices at specific point. In this case, we would like to include points from the three parameter regions in which qualitatively different behaviour occurs (see Fig 3.2a). For each point in the factorial design, the local sensitivity is estimated using the direct 


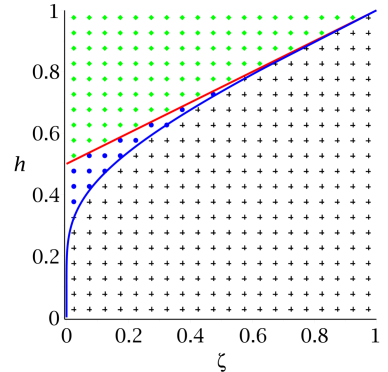

(a)

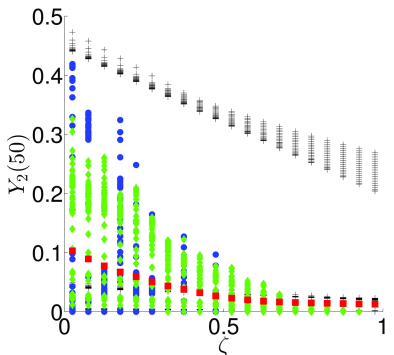

(c)

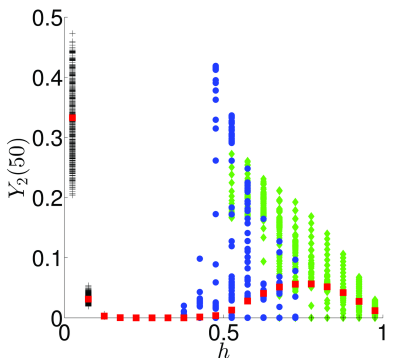

(e)

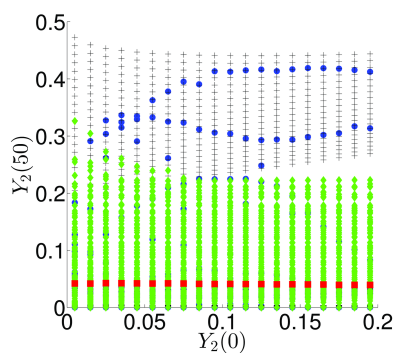

(g)

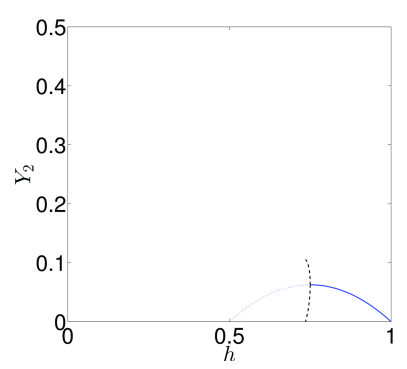

(b)

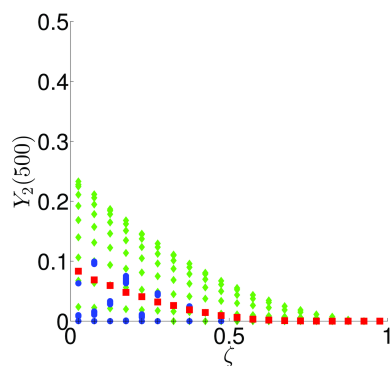

(d)

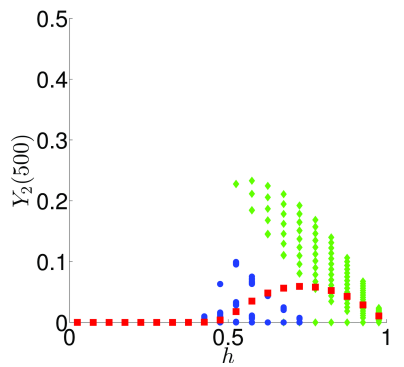

$(f)$

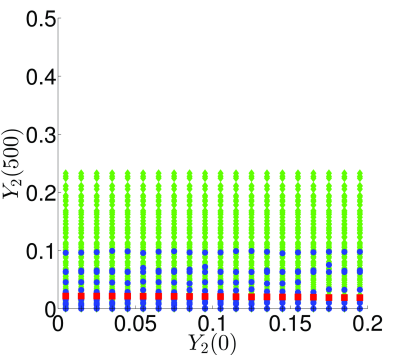

(h)

Figure 3.6: ABOS results displaying $Y_{2}(50)$ and $Y_{2}(500)$ for different parameter values. A full explanation is given in section 3.4.2. The parameters $Y_{1}(0)=0.9875, \gamma=1$., and $\kappa=1$. are fixed, whereas $\zeta$ and $h$ are sampled at equidistant steps. The colours correspond to the regions in the bifurcation diagram Fig 3.2a. 
differential method (see also Appendix 3.A). Variance-based sensitivity indices are computed around each point using Eq (3.10). The results are shown in Fig 3.7.

For the parameter region with bistability where $Y^{*}$ is stable (green), $h$ has a large peak around 1 and $\zeta$ around zero. From this it can be concluded that although in some points $\zeta$ is more influential, overall $h$ is the most influential parameter in this parameter region. This is a contrasting result compared to the results that are obtained using the Sobol' method, which indicates that $\zeta$ is overall the most influential parameter. The reason that the Sobol' method indicates $h$ as less influential is that larger values of $h$ have a negative effect on the output through lowering the steady state value (Fig 3.6f), but also has a positive effect because fewer model runs evolve to extinction. These two effects partly cancel each other out, resulting in a lower global sensitivity. Even though the aggregated sensitivity may be lower, $h$ is actually the most influential parameter in determining whether the steady state $Y$ is stable (Fig 3.6a), and it is also the most influential parameter in the steady state (Fig 3.7). The DELSA method allows us to discriminate between sensitivities in parameter regions with qualitatively different behaviour, and shows that indeed the ranking of sensitivities may differ between these regions. These results could not have been obtained through the Sobol' methodology, because this method assumes that the input parameters are independent. For the green region of bistability in Fig 3.2a, this is clearly not the case. Naive application of the Sobol' methodology to this parameter region leads to sensitivities that are not properly normalised as is shown in Appendix 3.E. Although the DELSA method is useful for showing how sensitivities vary between regions of the parameter space, it cannot show the effects of a tipping point directly because the local sensitivities are not well-defined in a tipping point. Thus, for this example we had to assume that information on the tipping points is already known. The sampling was done such that none of the sample points were in a tipping points, where the local sensitivities are not well-defined. For long simulation times the DELSA sensitivity indices may be computed analytically by using the derivatives of the steady state value (Appendix 3.C). 


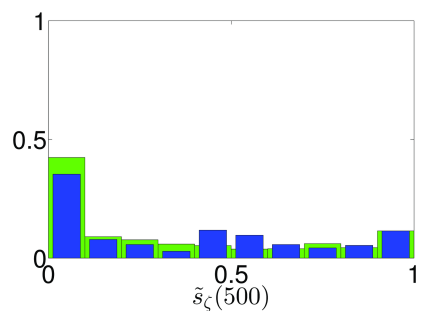

(a)

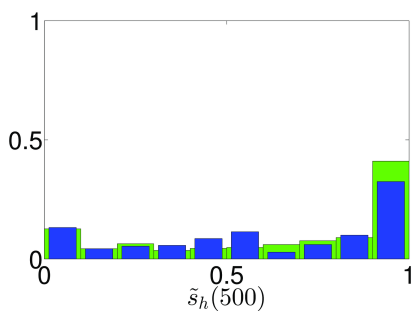

(b)

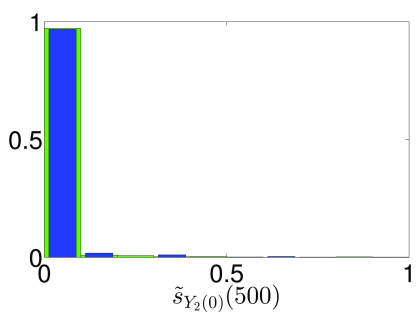

(c)

Figure 3.7: Histogram of DELSA sensitivity indices $\tilde{s}_{i}$ Eq (3.10) for $\zeta$ a, $h$ b and $Y_{2}(0)$ c at $t=500$, after all model runs have converged almost to their final behaviour. Parameters $Y_{1}(0)=0.9875, \gamma=1$., and $\kappa=1$. are fixed, whereas $\zeta, h$, and $Y_{2}(0)$ were varied using the factorial design. The different colours correspond to the regions in Fig 3.2a. To compare the different regions, each histogram was divided by the number of samples within the region. The results show that $h$ is overall the most influential parameter in the green region where the nontrivial steady state is stable. However, in some parts of these region $\zeta$ is more influential. In the blue region that corresponds to limit cycles, the spread in the sensitivity indices is large. 


\subsection{Discussion \& Conclusions}

The usefulness of simulation models for studying tipping points and resilience in ecological systems is limited by the availability of suitable methodologies for model analysis. Bifurcation analysis is the preferred methodology for detecting tipping points in deterministic models, but cannot be applied to other types of simulation models. The results in this paper show that sensitivity analysis methodologies can be useful to obtain information on tipping points in ecological models. Furthermore, it is shown that a combination of different methodologies for sensitivity analysis increases the amount of information on possible tipping points in ecological models. Below we discuss the possibilities and limitations of each of the applied methodologies in more detail.

Local sensitivity analysis is a useful methodology for assessing which parameters in ecological models are the most influential around a nominal point. This methodology considers only the linear response of the output. It is thus not well-suited for detecting tipping points. A local analysis can be extended as a one-factor-at-a-time (OFAT) analysis (Ten Broeke, Van Voorn and Ligtenberg 2016), in which the sensitivity of the model to a certain parameter is investigated by going stepwise through parameter space, where at each parameter value simulation is used to determine the model output. In effect this methodology can be considered as a discrete, brute force analogue to bifurcation analysis. Note, that since OFAT does not scan the full parameter space, it may not detect all tipping points. By including more than one parameter in the OFAT analysis the probability of detecting other tipping points (if there are any) increases. The costs of OFAT are typically not such that the inclusion of other parameters becomes prohibitively computationally expensive. In this paper, we show that OFAT can reveal separatrices and transcritical bifurcations. In Ten Broeke, Van Voorn and Ligtenberg (2016) it was shown that OFAT can be applied to detect tipping points in non-ODE models with a comparable level of success.

Global sensitivity analysis evaluates parameter sensitivities by aggregating model output across parameter space. Time-dependent global sensitivity analysis can be used to gain insight into the dynamics of a model and to suggest parameters for more detailed investigation. In our example, time-dependent global sensitivity analysis shows that the global sensitivities continue to show oscillations on long simulation times, which indicates that some samples display limit cycles. Given that other samples evolve to a stable steady state, this indicates the existence of a Hopf bifurcation. However, standard global sensitivity analysis methodologies do not consider qualitative changes of the underlying model behaviour. As a result, these methodologies can give misleading outcomes in the presence of tipping points (van Nes and Scheffer 2003). In our test-case the Sobol' indices indicate $\zeta$ as the most influential parameter. However, $h$ is more influential in determining whether the model converges to the positive steady state (Figs 3.2a and 3.6), and also on the value of the steady state (Fig 3.7). The reason that the Sobol' approach leads to a misleading conclusion is that an increase in $h$ has a positive effect on the mean output because fewer sample points converge to extinction, but also has a negative effect because value of the positive steady state is lowered. This information is revealed by applying a sampling design that allows for the calculation of (discrete) conditional means and that has sufficient coverage, e.g. the factorial design we use in this paper, combined with a proper graphical representation (Fig 3.6). Sparser sampling designs, such as the design in 
Saltelli et al. (2008) have the negative side-effect that critical information about tipping points is lost. If the goal is to locate tipping points, we thus recommend to adopt a proper sampling design and data representation. Ideally such a design would scan the entire parameter space, like the factorial design, but this is often not possible in practice due to the computational expense. For larger parameter spaces, a good sampling design may retain the characteristic of the ABOS method that the model output is evaluated at a number of values for certain (combinations) of parameters, while keeping all other parameters constant. Such an approach allows to (graphically) isolate the effects of the investigated parameters as in Fig 3.6 and gives better insight into how these combinations of parameters affect the output, including the possible presence of tipping points. A further limitation of the Sobol' method is its reliance on the assumption that the model parameters are independent. This assumption is not always reasonable for ecological models, where certain parameter combinations may not make sense in terms of the ecological assumptions behind the model, or when we want to quantify sensitivities separately for subregions of parameter space.

Hybrid methodologies of sensitivity analysis give a good overview of the global parameter sensitivities (Rakovec et al. 2014). Furthermore, these methodologies are well-suited for the computation of parameter sensitivities for subregions within the parameter space. Thus, if the approximate location of tipping points in parameter space is known, hybrid methodologies of sensitivity analysis can be used to identify influential parameters for separate types of model behaviour, as was done in Fig 3.7 for the Bazykin-Berezovskaya model. A limitation of hybrid methodologies is that they do not seem to reveal information about the location of tipping points. For that task a different approach should be followed.

The results in this paper show that global methodologies of sensitivity analysis may yield misleading results when naively applied across the full parameter space of a model that contains tipping points. When methods of bifurcation analysis are applicable, these can be used to detect bifurcations before global sensitivity analysis is applied. Should tipping points be found, then we suggest to separate parameter space (and possibly state space) based on the location(s) of the tipping point(s) and perform separate sensitivity analysis for the different regions. This way the obtained results are more informative regarding the sensitivity of the model to the different inputs. For models that cannot be analysed using bifurcation analysis, sensitivity analysis methodologies can yield some information on the possible existence of tipping points. Specifically, we suggest the use of OFAT as a starting point to gain information about (the location of) tipping points. Since OFAT does not scan the full parameter space, ideally it is supplemented by a global method of sensitivity analysis to investigate interaction effects. Here we used a factorial design, which scans the full parameter space, and combined with a graphical representation as in Fig 3.6, can also be used gain information about tipping points. We have shown that well-known methodology can still be applied to systems with tipping points - which are in fact the most interesting ones in ecology -, provided that they are combined with a thorough insight in the bifurcation structure of the system. In line with the 'curse of dimensionality' we also admit that a complete analysis of systems with a high number of dimensions still meets with some practical limitations and can be considered an open problem. This open issue is part of ongoing research. 


\section{Acknowledgements}

The research by GTB is financed by the IP/OP theme Complex Adaptive Systems at Wageningen University \& Research.

\section{A Direct differential method}

Local sensitivity indices Eq (3.2) for ODEs can be estimated using the direct differential method (Dickinson and Gelinas 1976, Richard et al. 2015). This method expresses each sensitivity index as an additional differential equation to be solved alongside the original ODE model. These equations are obtained by taking the time-derivative of the sensitivity index,

$$
\frac{d}{d t}\left(s_{i, j}\right)=\frac{d}{d t}\left(\frac{\partial Y_{j}}{\partial \theta_{i}}\right)=\frac{\partial}{\partial \theta_{i}}\left(\frac{d Y_{j}}{d t}\right),
$$

with $i=1,2, \ldots, N_{p}$ and $j=1,2, \ldots, N_{v}$.

For ODE models we have analytical expressions for the model equations $\frac{d Y_{j}}{d t}=$ $g_{j}(Y, \theta, t)$, which are inserted in Eq (3.18). Considering that $Y_{j}=Y_{j}(\theta, t)$, we use the chain rule for differentiation to rewrite $\mathrm{Eq}(3.18)$,

$$
\begin{aligned}
\frac{d}{d t}\left(s_{i, j}\right) & =\frac{\partial g_{j}}{\partial \theta_{i}}+\sum_{m=1}^{N_{v}} \frac{\partial g_{j}}{\partial Y_{m}} \frac{\partial Y_{m}}{\partial \theta_{i}} \\
& =\frac{\partial g_{j}}{\partial \theta_{i}}+\sum_{m=1}^{N_{v}} \frac{\partial g_{j}}{\partial Y_{m}} s_{i, m}
\end{aligned}
$$

with the summation running over all state variables. Since at $t=0$ the state variables are given by their initial conditions, we have $s_{i, j}(0)=1$ for the sensitivity to the initial condition of $Y_{j}$, and $s_{i, j}(0)=0$ for other parameters. Eq (3.20) is solved numerically alongside the original ODE model to obtain the local sensitivity indices as a function of time.

It can be shown that in the case the original ODE model is stable, the extended system Eq (3.20) is also stable. This was shown in Fig 3.3a, for the nominal point. However, when the original system is unstable and possesses a periodic attractor (in the blue region in Fig 3.2a) the sensitivity index to the initial condition of $Y_{j}$ shows, just as the original state variables, a periodic behaviour while the other sensitivity indices grow in time oscillatory without bounds when time goes to infinity. Note that the effects of these oscillations are not visible in Fig 3.4b because none of the grid points was in the blue region in Fig 3.2a.

\section{B Local sensitivity analysis of the Bazykin- Berezovskaya model}

The local sensitivity indices of the Bazykin-Berezovskaya model Eq (3.11) are obtained as a function of time using the direct differential method (Appendix 3.A). Taking the 
derivative of the model equations Eq (3.11) with respect to the parameter $\zeta$, while noting that the state variables are functions of the parameters, yields

$$
\begin{aligned}
\frac{d}{d t}\left(\frac{\partial Y_{1}}{\partial \zeta}\right)= & \frac{\partial Y_{1}}{\partial \zeta}\left(Y_{1}-\zeta\right)\left(\kappa-Y_{1}\right)+Y_{1}\left(\frac{\partial Y_{1}}{\partial \zeta}-1\right)\left(\kappa-Y_{1}\right)- \\
& Y_{1}\left(Y_{1}-\zeta\right) \frac{\partial Y_{1}}{\partial \zeta}-\frac{\partial Y_{1}}{\partial \zeta} Y_{2}-Y_{1} \frac{\partial Y_{2}}{\partial \zeta} \\
\frac{d}{d t}\left(\frac{\partial Y_{2}}{\partial \zeta}\right)= & \gamma \frac{\partial Y_{1}}{\partial \zeta} Y_{2}+\gamma\left(Y_{1}-h\right) \frac{\partial Y_{2}}{\partial \zeta}
\end{aligned}
$$

Similarly, for $h$

$$
\begin{aligned}
\frac{d}{d t}\left(\frac{\partial Y_{1}}{\partial h}\right)= & \frac{\partial Y_{1}}{\partial h}\left(Y_{1}-\zeta\right)\left(\kappa-Y_{1}\right)+Y_{1} \frac{\partial Y_{1}}{\partial h}\left(\kappa-Y_{1}\right)- \\
& Y_{1}\left(Y_{1}-\zeta\right) \frac{\partial Y_{1}}{\partial h}-\frac{\partial Y_{1}}{\partial h} Y_{2}-Y_{1} \frac{\partial Y_{2}}{\partial h} \\
\frac{d}{d t}\left(\frac{\partial Y_{2}}{\partial h}\right)= & \gamma\left(\frac{\partial Y_{1}}{\partial h}-1\right) Y_{2}+\gamma\left(Y_{1}-h\right) \frac{\partial Y_{2}}{\partial h}
\end{aligned}
$$

and for $Y_{2}(0)$

$$
\begin{aligned}
\frac{d}{d t}\left(\frac{\partial Y_{1}}{\partial Y_{2}(0)}\right)= & \frac{\partial Y_{1}}{\partial Y_{2}(0)}\left(Y_{1}-\zeta\right)\left(\kappa-Y_{1}\right)+Y_{1} \frac{\partial Y_{1}}{\partial Y_{2}(0)}\left(\kappa-Y_{1}\right)- \\
& Y_{1}\left(Y_{1}-\zeta\right) \frac{\partial Y_{1}}{\partial Y_{2}(0)}-\frac{\partial Y_{1}}{\partial Y_{2}(0)} Y_{2}-Y_{1} \frac{\partial Y_{2}}{\partial Y_{2}(0)} \\
\frac{d}{d t}\left(\frac{\partial Y_{2}}{\partial Y_{2}(0)}\right)= & \gamma \frac{\partial Y_{1}}{\partial Y_{2}(0)} Y_{2}+\gamma\left(Y_{1}-h\right) \frac{\partial Y_{2}}{\partial Y_{2}(0)} .
\end{aligned}
$$

Since the state variables at $t=0$ are given by their initial conditions, the initial conditions of Eqs (3.21-3.25) are equal to zero. For Eq (3.26) we have $\frac{\partial Y_{2}(0)}{\partial Y_{2}(0)}=1$. Eqs (3.21-3.26) are then solved numerically alongside the model equations to obtain the sensitivity indices as a function of time for any given set of parameter values.

\section{C Distribution of local sensitivity indices}

The local sensitivity of steady state value $Y_{2}^{*} \mathrm{Eq}(3.12)$ with respect to model parameter $h$ reads

$$
s_{h}^{*}=\frac{\partial Y_{2}^{*}}{\partial h}=1+\zeta-2 h
$$

Here we have set all parameters at their nominal values except for $\zeta$ and $h$. Eq (3.27) measures the sensitivity around a point in parameter space. To measure the sensitivity over a larger region of parameter space we use the expectation value and variance of 
Eq (3.27) over this region. For the stable steady state region (green) in Fig 3.2a we have for the expectation value $E\left(s_{h}^{*}\right)$

$$
E\left(s_{h}^{*}\right)=\int_{h=1 / 2}^{h=1} \int_{\zeta=0}^{\zeta=2 h-1} 4 s_{h}^{*} d \zeta d h,
$$

where the factor 4 is a normalisation factor to ensure that the total probability is equal to one. This normalisation factor is equal to one divided by the area of the surface over which we integrate. For the variance $V\left(s_{h}^{*}\right)$ we have

$$
V\left(s_{h}^{*}\right)=\int_{h=1 / 2}^{h=1} \int_{\zeta=0}^{\zeta=2 h-1} 4\left(s_{h}^{*}-E\left(s_{h}^{*}\right)\right)^{2} d \zeta d h .
$$

The local sensitivity of the steady state value $Y_{2}^{*}$ with respect to model parameter $\zeta$ reads

$$
s_{\zeta}^{*}=\frac{\partial Y_{2}^{*}}{\partial \zeta}=h-\kappa .
$$

We have for the expectation value $E\left(s_{\zeta}^{*}\right)$

$$
E\left(s_{\zeta}^{*}\right)=\int_{h=1 / 2}^{h=1} \int_{\zeta=0}^{\zeta=2 h-1} 4 s_{\zeta}^{*} d \zeta d h
$$

and for the variance $V\left(s_{\zeta}^{*}\right)$

$$
V\left(s_{\zeta}^{*}\right)=\int_{h=1 / 2}^{h=1} \int_{\zeta=0}^{\zeta=2 h-1} 4\left(s_{\zeta}^{*}-E\left(s_{\zeta}^{*}\right)\right)^{2} d \zeta d h
$$

Instead of the local sensitivity index of Eq (3.27), the expectation value and variance may be computed for the DELSA sensitivity indices, by normalising the partial derivative according to Eq (3.10). The calculation of the expectation value and the variance then proceeds in the same way.

\section{D Variance decomposition in Sobol' method}

The variance decomposition Eq (3.4) follows from a decomposition of the model in terms of the different model parameters (Sobol' 2001, Saltelli et al. 2008). We write the model as

$$
Y=f(\theta)
$$

where $\theta=\left(\theta_{1}, \ldots, \theta_{N_{p}}\right) \in I^{N_{p}}$ and $I^{N_{p}}$ the $N_{p}$-dimensional unit hypercube. We assume uniform distributions between 0 and 1 for all parameters $\left(\theta_{i} \sim U(0,1)\right)$ and decompose the model output as 


$$
f\left(\theta_{1}, \theta_{2}, \ldots, \theta_{N_{p}}\right)=f_{0}+\sum_{i} f_{i}\left(\theta_{i}\right)+\sum_{i<l} f_{i, l}\left(\theta_{i}, \theta_{l}\right)+\ldots+f_{1,2, \ldots N_{p}}\left(\theta_{1}, \theta_{2}, \ldots, \theta_{N_{p}}\right)
$$

The decomposition of Eq (3.34) is not unique (Saltelli et al. 2008). If the decomposition is chosen such that all the terms have zero mean,

$$
\int f_{i}\left(\theta_{i}\right) d \theta_{i}=0
$$

then it follows (Sobol' 2001) that all the pairs are orthogonal,

$$
\int f_{i}\left(\theta_{i}\right) f_{l}\left(\theta_{l}\right) d \theta_{i} d \theta_{l}=0
$$

Squaring and integrating Eq (3.34) then yields the variance decomposition,

$$
\int f(\theta)^{2} d \theta-f_{0}^{2}=\sum_{i} \int f_{i}^{2} d \theta_{i}+\sum_{i<l} \int f_{i, l}^{2} d \theta_{i} d \theta_{l}+\int f_{1,2, \ldots, N_{p}}^{2} d \theta_{1} d \theta_{2} \ldots d \theta_{N_{p}}
$$

or

$$
V\left(Y_{j}\right)=\sum_{i} V_{i}+\sum_{i<l} V_{i, l}+\ldots+V_{1,2, \ldots, N_{p}}
$$

Thus, the total variance is decomposed into terms that can be attributed to (combinations of) model parameters. It follows from Eq (3.35) that the terms of Eq (3.34) have an interpretation as conditional expectations of the model output,

$$
\begin{aligned}
f_{0} & =E(Y), \\
f_{i} & =E\left(Y \mid \theta_{i}\right)-f_{0}, \\
f_{i, l} & =E\left(Y \mid \theta_{i}, \theta_{l}\right)-f_{i}-f_{l}-f_{0} .
\end{aligned}
$$

The terms of the variance decomposition Eq (3.38) can therefore be expressed as

$$
V_{i}=V_{\theta_{i}}\left(E_{\theta_{\sim i}}\left(Y_{j} \mid \theta_{i}\right)\right)
$$

which gives the commonly used measure for the first-order sensitivity index Eq (3.7). Expressions for the higher order sensitivity indices are derived in the same way. 


\section{E Sobol' indices in the steady state}

To illustrate the computation of the Sobol' indices and show the limitations of the methodology in case of parameter dependencies, we calculate here the first- and totalorder sensitivity indices Eqs (3.7-3.8) for the steady state Eq (3.12) of the BazykinBerezovskaya model. As parameter range for $h$ and $\zeta$ we use the green region of bistability in Fig 3.2a. We assume that the initial conditions are chosen such that the model evolves to the positive steady state $Y^{*}$ and keep all other parameters fixed at the nominal values. Note that the chosen parameter range imposes a dependency between the parameters $h$ and $\zeta$. As a result the normalisation of the sensitivity indices Eq (3.4) does not hold for this example.

The steady state value of $Y_{2}$ is given by Eq (3.12). Since the global sensitivity indices are normalised with respect to the total variance, we first calculate the mean and variance of $Y_{2}^{*}$ over the stable steady state region (green) in Fig 3.2a,

$$
\begin{aligned}
E\left(Y_{2}^{*}\right) & =\int_{\zeta=0}^{\zeta=1} \int_{h=(\zeta+1) / 2}^{h=1} Y_{2}^{*} P_{h}(h) P_{\zeta}(\zeta) d h d \zeta \\
V\left(Y_{2}^{*}\right) & =\int_{\zeta=0}^{\zeta=1} \int_{h=(\zeta+1) / 2}^{h=1}\left(Y_{2}^{*}-E\left(Y_{2}^{*}\right)\right)^{2} P_{h}(h) P_{\zeta}(\zeta) d h d \zeta
\end{aligned}
$$

where the probability density functions $P_{h}=\frac{2}{1-\zeta}$ and $P_{\zeta}=1$ describe a uniform probability density over the region. To obtain the expression for $P_{h}$, note that for any given value of $\zeta$, the length of the interval of $h$ within the stable steady state region is equal to $\frac{1-\zeta}{2}$. The corresponding uniform probability density is given by 1 divided by this length.

To obtain the first-order sensitivity index of $\zeta$ we first compute the expectation value of $Y_{2}^{*}$ while keeping $\zeta$ fixed,

$$
E_{h}\left(Y_{2}^{*} \mid \zeta\right)=\int_{h=(\zeta+1) / 2}^{h=1} Y_{2}^{*} P_{h}(h) d h,
$$

with a subscript to denote the variable over which the expectation value is evaluated. Observe that the expectation $E_{h}\left(Y_{2}^{*} \mid \zeta\right)$ is still a function of $\zeta$. The variance $V_{\zeta}\left(E_{h}\left(Y_{2}^{*} \mid \zeta\right)\right)$ is computed over the range of $\zeta$. We first calculate the mean

$$
E_{\zeta}\left(E_{h}\left(Y_{2}^{*} \mid \zeta\right)\right)=\int_{\zeta=0}^{\zeta=1} E_{h}\left(Y_{2}^{*} \mid \zeta\right) P_{\zeta}(\zeta) d \zeta
$$

and then the variance

$$
V_{\zeta}\left(E_{h}\left(Y_{2}^{*} \mid \zeta\right)\right)=\int_{\zeta=0}^{\zeta=1}\left(E_{h}\left(Y_{2}^{*} \mid \zeta\right)-E_{\zeta}\left(E_{h}\left(Y_{2}^{*} \mid \zeta\right)\right)\right)^{2} P_{\zeta}(\zeta) d \zeta
$$

This expression is normalised with respect to the total variance to obtain the firstorder sensitivity index. 
Similarly, for the first-order sensitivity of $h$, we have

$$
E_{\zeta}\left(Y_{2}^{*} \mid h\right)=\int_{\zeta=0}^{\zeta=2 h-1} Y_{2}^{*} \hat{P}_{\zeta}(\zeta) d \zeta
$$

with $\hat{P}_{\zeta}=\frac{1}{2 h-1}$ to ensure a uniform distribution over the region. For the variance of this expectation value we then have

$$
\begin{aligned}
& E_{h}\left(E_{\zeta}\left(Y_{2}^{*} \mid h\right)\right)=\int_{h=\frac{1}{2}}^{h=1} E_{\zeta}\left(Y_{2}^{*} \mid h\right) \hat{P}_{h}(h) d h \\
& V_{h}\left(E_{\zeta}\left(Y_{2}^{*} \mid h\right)\right)=\int_{h=\frac{1}{2}}^{h=1}\left(E_{\zeta}\left(Y_{2}^{*} \mid h\right)-E_{h}\left(E_{\zeta}\left(Y_{2}^{*} \mid h\right)\right)\right)^{2} \hat{P}_{h}(h) d h
\end{aligned}
$$

where $\hat{P}_{h}=2$ is a uniform probability distribution between $h=\frac{1}{2}$ and $h=1$. This expression is inserted into Eq (3.7). The derivation of the total-order indices Eq (3.8) is not given here, but is similar to the first-order indices.

The resulting values for the first- and total-order sensitivities are given in Table 3.3. The outcomes are difficult to interpret because Eq (3.4) holds only for independent parameters. For dependent parameters, the sensitivity indices are thus not properly normalised, and are not bound between 0 and 1 . In the present case, we find that $h$ has the largest first-order sensitivity index, but the value is larger than one, which is normally not possible since the sensitivity indices are expressed as proportions of the total variance that are explained by a certain parameter. Furthermore, both of the total-order sensitivities are smaller than the corresponding sensitivities, which does not make sense given that the total-order sensitivity includes higher-order effects in addition to first-order effects.

To apply the Sobol' method to the stable steady state region (green) of Fig 3.2a without violating the assumption of independent parameters, we might perform a parameter transformation that transforms the triangular region into a square region (Mara and Tarantola 2012). However, since the sensitivity indices are then computed for the transformed independent parameters instead of the original parameters, it is difficult to interpret the outcomes in terms of the original model parameters. This approach therefore does not really remedy the limitations of the Sobol' method.

Instead of considering only the steady state region, the Sobol' method can be applied to the entire parameter range shown in Fig 3.2a. The parameters $h$ and $\zeta$ are then independent and ranging between 0 and 1, thus ensuring proper normalisation of the sensitivity indices. These sensitivity indices cannot be calculated analytically because we have no analytical expression for the model output for the blue region in Fig 3.2a. This calculation was therefore done numerically using both the factorial design and the design by Saltelli et al. (2010). The results at $t=10, t=50$, and $t=200$ are given in Table 3.4 and are discussed in Section 3.4.2.

\section{F Sampling method for the Sobol' indices}

The methodology described by Saltelli et al. (2010) is a numerical method to estimate Eqs (3.7-3.8), based on the generation of two sample matrices, $\mathbf{A}$ and B. Each contains 
the same number of sample points $N_{s}$, drawn independently from the probability density functions of the parameters. Then $N_{p}$ recombination matrices $\mathbf{A}_{\mathbf{B}}{ }^{(i)}\left(\mathbf{B}_{\mathbf{A}}{ }^{(i)}\right)$ are generated from the two sampling matrices, where $\mathbf{A}_{\mathbf{B}}{ }^{(i)}\left(\mathbf{B}_{\mathbf{A}}{ }^{(i)}\right)$ is identical to $\mathbf{A}$ (B) except for its $i^{\text {th }}$ column, that is taken from $\mathbf{B}(\mathbf{A})$. The model is then run for each sample point in the sample matrices and the recombination matrices, generating a vector of output values. The computational costs of generating these data is thus $2 N_{s}\left(N_{p}+1\right)$ model runs.

Writing $Y_{A}$ for the vector that contains the output values of sample matrix $A$, the first-order index can be estimated as (Saltelli et al. 2010)

$$
S_{i} \approx S_{i}^{A}=\frac{1}{V(Y)}\left(\frac{1}{N_{s}} Y_{A} \cdot Y_{B_{A}}-E(Y)^{2}\right),
$$

where $\cdot$ denotes the inner product and the index $j$ is dropped for convenience. One may also use the alternative

$$
S_{i} \approx S_{i}^{B}=\frac{1}{V(Y)}\left(\frac{1}{N_{s}} Y_{B} \cdot Y_{A_{B}}-E(Y)^{2}\right),
$$

or the sum of Eqs (3.51-3.52) divided by two (Glen and Isaacs 2012)

$$
S_{i} \approx \frac{1}{2}\left(S_{i}^{A}+S_{i}^{B}\right)
$$

The total-order sensitivity indices Eq (3.8) are estimated as (Saltelli et al. 2010),

$$
\tilde{S}_{i} \approx \tilde{S}_{i}^{A}=1-\frac{1}{V(Y)}\left(\frac{1}{N_{s}} Y_{A} \cdot Y_{A_{B}}-E(Y)^{2}\right)
$$

or alternatively

$$
\tilde{S}_{i} \approx \tilde{S}_{i}^{B}=1-\frac{1}{V(Y)}\left(\frac{1}{N_{s}} Y_{B} \cdot Y_{B_{A}}-E(Y)^{2}\right) .
$$

Also here we follow the line of Glen and Isaacs (2012) and combine

$$
\tilde{S}_{i} \approx \frac{1}{2}\left(\tilde{S}_{i}^{A}+\tilde{S}_{i}^{B}\right)
$$

The sample mean $E\left(Y_{j}\right)$ and variance $V\left(Y_{j}\right)$ can be approximated in different ways. In the limit case

$$
E(Y) \approx E\left(Y_{A}\right)
$$

which is a commonly used estimator (Sobol' 2001). One can also use the alternative (Saltelli et al. 2010) 


$$
E(Y) \approx E\left(Y_{B}\right)
$$

However, for limited sample sizes a better estimator of the sample mean is considered to be (Saltelli et al. 2010) the square root of

$$
E(Y)^{2} \approx \frac{1}{N_{s}} Y_{A} \cdot Y_{B}
$$

In line with Eq (3.53) proposed by Glen and Isaacs (2012) we use the sum of Eqs (3.57$3.58)$

$$
E(Y) \approx \frac{1}{2}\left(E\left(Y_{A}\right)+E\left(Y_{B}\right)\right)
$$

and for the variance

$$
V(Y) \approx \frac{1}{2}\left(V\left(Y_{A}\right)+V\left(Y_{B}\right)\right)
$$

The choice of estimator for sample mean, variance, first and total order sensitivity can lead to different results, as can the selected sampling method. Alternative estimators for $S_{i, j}$ and $\tilde{S}_{i, j}$ are discussed by Saltelli et al. (2010), Glen and Isaacs (2012), while alternative methods are evaluated by Saltelli et al. (2010). 
Table 3.4: Sample mean, variance, first-order $S_{i}$ and total order $\tilde{S}_{i}$ variance-based sensitivities of $Y_{2}$ at $t=10 \mathrm{a}, t=50(\mathrm{~b})$, and $t=200(\mathrm{c})$, expressed as percentages. Parameters $Y_{1}(0), \gamma=1$., and $\kappa=1$. are fixed. Parameters $\zeta$ and $h$ are sampled uniformly between 0 and 1., and $Y_{2}(0)$ between 0 and 0.2 . First columns: sampling from a $n \times n \times n$ grid, with $n$ indicated. Last columns: using estimators $\mathrm{Eq}$ (3.53), Eq (3.56), Eq (3.60), and Eq (3.61) for different $N_{s}$. CC indicates computational costs measured by number of performed model runs.

(a) $t=10$

\begin{tabular}{lllllll}
\hline & $n=10$ & $n=20$ & $n=40$ & $N_{s}=500$ & $N_{s}=10^{3}$ & $N_{s}=2 \cdot 10^{3}$ \\
\hline$C C$ & 1000 & 8000 & 64000 & 4000 & 8000 & 16000 \\
\hline$E\left(Y_{j}\right)$ & 0.189 & 0.191 & 0.192 & 0.217 & 0.218 & 0.227 \\
$V\left(Y_{j}\right)$ & 0.053 & 0.057 & 0.059 & 0.055 & 0.049 & 0.047 \\
$S_{\zeta}$ & 10.72 & 9.395 & 8.874 & 4.307 & 7.301 & 10.08 \\
$S_{h}$ & 92.66 & 88.66 & 86.77 & 68.28 & 74.88 & 88.10 \\
$S_{y_{2}(0)}$ & 2.207 & 1.936 & 1.796 & 1.816 & 2.646 & 3.974 \\
$\tilde{S}_{\zeta}$ & 12.29 & 12.04 & 11.86 & 26.96 & 17.53 & 4.828 \\
$\tilde{S}_{h}$ & 87.84 & 88.84 & 89.24 & 88.43 & 88.31 & 85.70 \\
$\tilde{S}_{y_{2}(0)}$ & 3.666 & 4.295 & 4.410 & 22.05 & 13.82 & 1.589 \\
\hline
\end{tabular}

(b) $t=50$

\begin{tabular}{lllllll}
\hline & $n=10$ & $n=20$ & $n=40$ & $N_{s}=500$ & $N_{s}=10^{3}$ & $N_{s}=2 \cdot 10^{3}$ \\
\hline$C C$ & 1000 & 8000 & 64000 & 4000 & 8000 & 16000 \\
\hline$E\left(Y_{j}\right)$ & .0323 & .0412 & .0450 & .034 & .031 & .034 \\
$V\left(Y_{j}\right)$ & .0030 & .0076 & .0120 & .0053 & .0048 & .0050 \\
$S_{\zeta}$ & 29.73 & 14.74 & 9.960 & 24.45 & 18.33 & 16.62 \\
$S_{h}$ & 39.10 & 68.45 & 77.92 & 42.75 & 37.55 & 39.23 \\
$S_{y_{2}(0)}$ & .102 & .016 & .002 & -.275 & -.247 & -3.084 \\
$\tilde{S}_{\zeta}$ & 64.12 & 34.64 & 23.86 & 60.53 & 62.76 & 59.72 \\
$\tilde{S}_{h}$ & 72.55 & 85.87 & 90.25 & 74.27 & 81.65 & 82.38 \\
$\tilde{S}_{y_{2}(0)}$ & 2.993 & 1.553 & 1.197 & 7.221 & 5.209 & 3.431 \\
\hline
\end{tabular}

(c) $t=200$

\begin{tabular}{lllllll}
\hline & $n=10$ & $n=20$ & $n=40$ & $N_{s}=500$ & $N_{s}=10^{3}$ & $N_{s}=2 \cdot 10^{3}$ \\
\hline$C C$ & 1000 & 8000 & 64000 & 4000 & 8000 & 16000 \\
\hline$E\left(Y_{j}\right)$ & .0288 & .0313 & .0345 & .0276 & .310 & .0292 \\
$V\left(Y_{j}\right)$ & .005 & .0006 & .0006 & .00558 & .00669 & .0625 \\
$S_{\zeta}$ & 33.90 & 33.90 & 31.37 & 19.8 & 17.4 & 15.2 \\
$S_{h}$ & 14.05 & 10.58 & 17.33 & 58.2 & 57.5 & 49.2 \\
$S_{y_{2}(0)}$ & .047 & .121 & .036 & -1.93 & -1.40 & -1.01 \\
$\tilde{S}_{\zeta}$ & 87.04 & 89.56 & 82.93 & 40.7 & 42.2 & 49.0 \\
$\tilde{S}_{h}$ & 69.00 & 67.37 & 64.07 & 78.9 & 84.4 & 83.9 \\
$\tilde{S}_{y_{2}(0)}$ & .928 & 6.407 & 4.442 & 3.25 & -3.66 & 2.25 \\
\hline
\end{tabular}





\section{Chapter 4}

\section{Which sensitivity analysis method should I use for my Agent-based Model?}

G.A. ten Broeke, G.A.K. van Voorn, A. Ligtenberg.

Journal of Artificial Societies and Social Simulation, 19(1). 
Existing methodologies of sensitivity analysis may be insufficient for a proper analysis of Agent-based Models (ABMs). Most ABMs consist of multiple levels, contain various nonlinear interactions, and display emergent behaviour. This limits the information content that follows from the classical sensitivity analysis methodologies that link model output to model input. In this paper we evaluate the performance of three well-known methodologies for sensitivity analysis of ABMs. The three methodologies are OFAT (one-factor-at-a-time), and proportional assigning of output variance by means of model fitting and by means of Sobol' decomposition. The methodologies are applied to an ABM case study of limited complexity consisting of free-roaming and procreating agents that make harvest decisions with regard to a diffusing renewable resource. We find that each methodology has its own merits and yields useful information, yet none of them provide a complete picture of model behaviour. We recommend OFAT as the starting point for sensitivity analysis of an $\mathrm{ABM}$, for its use in uncovering the mechanisms and patterns that the $\mathrm{ABM}$ produces.

\subsection{Introduction}

During the last decade Agent Based Models (ABMs) have become a standard technique in the toolbox of scholars interested in simulating and understanding complex adaptive systems such as land use systems (Parker et al. 2003, Bousquet and Le Page 2004, Matthews et al. 2007). Still missing are solid methods to explore the behaviour of an ABM and assess its relation with the empirical world (Crooks et al. 2008, Filatova et al. 2013). For ABMs to be useful as tools to learn about and understand complex adaptive systems it is essential to know the effects of different configurations of system components on global and emergent outcomes. These insights are especially crucial when one is interested in studying the conditions under which systems can become critical, i.e. when resilience and when tipping points can occur, for instance, when one wants to obtain early warning signals.

In order to explore the behaviour of models sensitivity analysis is a commonly used tool, at least in the field of traditional modelling. There exists a plethora of standardized methodologies for performing sensitivity analysis (Saltelli et al. 2004b, 2008, Hamby 1994, Cariboni et al. 2007, Thiele et al. 2014). However it is unclear to what extent these methodologies are suitable for the type of systems commonly represented by ABMs. It is important to observe though that all sensitivity analysis methodologies have a statistical underpinning. It is because of the statistical approach there is no mechanistic focus, and results obtained via sensitivity analysis always require an interpretation by model users in the context of the model application.

ABMs are characterized by several properties that make the application of any statistical approach, and thus most standard sensitivity analysis methods, a nontrivial matter. These properties are (Windrum et al. 2007, Gilbert 2008, Macal and North 2010)

- The existence of multiple levels. There is at least a micro-level consisting of individual and autonomous agents, and a macro-level environment that influences 
and is influenced by the agents. This implies there are also at least two distinct steps going from input to output;

- The existence of many and often nonlinear interactions. This suggests that relations between input and output may be nonlinear as well;

- The existence of emergent properties, i.e. patterns that are not predicted a priori based on the individual rules describing either the agent or the environment. Such patterns are for example path dependencies, adaptation, and tipping points. This also suggests that the relations between input and output may be nonlinear, and, additionally, may change over time.

It is for this reason that a straightforward application of classical methodologies for sensitivity analysis to ABMs will not always yield sufficient information required by the model user for model evaluation. The main complicating factor is that for most, if not all, complex adaptive systems the interactions and relations between system components are self-organizing processes. Relations between global system states (i.e., the outputs) and the states of the individual system components (i.e., the inputs) are not pre-defined like in traditional linear models. Different system states might be possible under similar parameter regimes, while completely different parameter regimes might yield similar system behaviour. This means that a standard sensitivity analysis performed on an ABM has only limited explanatory power. Developing targeted methods of sensitivity analysis has therefore rightly been identified as a key challenge for Agent-based Modelling (Crooks et al. 2008, Filatova et al. 2013, Thiele et al. 2014). In turn, the analysis, calibration, and validation of ABMs also remain pivotal issues (Brown et al. 2005, Fagiolo et al. 2007, Windrum et al. 2007, Crooks et al. 2008, Filatova et al. 2013).

In this paper we explore the potential of three well-known methodologies for sensitivity analysis to obtain information that is useful for a) assessing the implementation of an ABM and b) understanding the behaviour of the represented system. The three methodologies are described in Section 4.3. To have a measure of quantification of the potential of the elected methodologies, three goals have been selected which are typical for ABM applications. These goals are explained in 4.2. In 4.4 we present a case study model to apply the sensitivity analysis methodologies to. The details of the model are given in 4.A in ODD format. The choice of the case study is justified by the relatively low complexity of the model, which limits computational costs, while the model contains all the essential ingredients of an ABM. 4.5 gives an overview of the main results. 4.6 finalizes with the discussion.

\subsection{Selected goals for performing sensitivity analysis}

We have selected three application goals for sensitivity analysis which are common for ABM research. These are

- To gain insight in how patterns and emergent properties are generated in the ABM;

- To examine the robustness of emergent properties; 
- To quantify the variability in ABM outcomes resulting from model parameters.

To better understand dynamics and emergent patterns found in the real-world systems that are described by ABMs it is crucial to first understand how they are generated in the model. Given the complexity of many ABMs, understanding the model dynamics is typically not a straightforward task. Sensitivity analysis can help in this task because the effects of parameter changes on the model outcomes contain clues about the model dynamics underlying these outcomes (Ligmann-Zielinska et al. 2014). For example, revealing whether these effects are linear, non-linear, or push the system into an entirely different state by causing a tipping point yields valuable information on how a parameter affects the dynamics of the system.

A second motivation for performing sensitivity analysis is to examine the robustness of the model outcomes with respect to changes of the parameter values (Leamer 1983, 2010, Axtell 1999). A range of model parameter values corresponds to a range of assumptions that leads to certain inferences (i.e. model outcomes). For these inferences to be credible, however, they should not depend on a narrow and uncertain set of assumptions. It is therefore important to show that the inferences are robust to parameter changes. This is particularly relevant if the model attempts to explain a phenomenon that occurs under a wide range of circumstances in reality. For example, the well-known Schelling model (Schelling 1971) examines the emergence of patterns of ethnic segregation. The model shows that patterns of segregation emerge from a few simple assumptions on the movement of agents in a spatial environment. Furthermore, this emergence is robust to changes in the parameter values and movement rules of the agents (Gilbert 2002). This robustness adds to the credibility of the model, given the observation that segregation in reality occurs in different places and under various circumstances. A third and more traditional use of sensitivity analysis is to quantify the uncertainty of the model outcomes that is caused by parameter uncertainties (Hamby 1994, Saltelli et al. 2008). There are various sources that contribute to uncertainty of the model outcomes. One of these sources is uncertainty in the parameter set. We typically assign values to the model parameters based on measurements or expert judgements, but there is uncertainty in these values. The uncertainty of the parameter set causes uncertainty in the model outcomes. Sensitivity analysis quantifies this output variability and decomposes it into terms that are attributed to different parameters. This allows the user to rank the input uncertainties in terms of their contribution to output uncertainty. This ranking can for example be used to decrease the output uncertainty by decreasing the uncertainty of the parameter set.

\subsection{Selected methodologies for sensitivity analysis}

Many methodologies have been developed to perform sensitivity analysis (for an overview see for instance (Saltelli et al. 2004b, 2008, Cariboni et al. 2007)). Here we have selected three methodologies. These are

- Sensitivities based on one-factor-at-a-time (OFAT);

- Sensitivities based on model-free output variance decomposition;

- Sensitivities based on model-based output variance decomposition. 
These methodologies have been selected because they are commonly available in statistical packages (e.g. Thiele et al. (2014)), while they have proven to be very popular since they can be applied rather straightforwardly with limited statistical and computational efforts. We discuss the three methodologies in more detail below.

\subsubsection{One-factor-at-a-time (OFAT)}

OFAT sensitivity analysis essentially consists of selecting a base parameter setting (nominal set) and varying one parameter at a time while keeping all other parameters fixed (hence it is referred to as a local method). An important use of OFAT is to reveal the form of the relationship between the varied parameter and the output, given that all other parameters have their nominal values. For example, it shows whether the response is linear or nonlinear, or whether there are tipping points where the output responds drastically to a small parameter change. By showing these relationships, OFAT can yield understanding of model mechanisms. For this purpose, we vary each parameter over a number of points within a wide range and plot the resulting output.

Note that One-at-a-time (OAT) is traditionally used to estimate sensitivity measures in the form of partial derivatives of the model outcomes with respect to input parameters (e.g. Cariboni et al. (2007)). This estimation is based on the effect of small deviations from the nominal parameter values on the model outcomes. OFAT differs from OAT in that we examine the model response over a wide range for each parameter. The slope of the output graph at some parameter value roughly estimates the partial derivative at that point. A more accurate estimate would require a smaller step size in the parameter values and a large number of replicate runs to account for stochastic effects. However, such an estimate does not directly contribute towards our aim of showing the form of the relationship between the parameter and the output. We therefore do not explicitly compute these partial derivatives.

\subsubsection{Global sensitivity analysis}

Global sensitivity analysis (Saltelli et al. 2008) aims to ascertain interaction effects by sampling the model output over a wide range of parameter values. Many samples (i.e. specific parameter settings, usually determined randomly) are drawn from the parameter space, and the corresponding model outcomes are combined in a statistical measure, namely the variance of the output of the model. The sensitivity to a parameter is then measured as the proportion of the model variance that can be explained by changes in that parameter. Since the total model variance is used to normalise the sensitivities, the sensitivities of different parameters can be compared directly. There are two common ways to decompose the output variance:

- By using a regression model to fit the model output to model parameters, in a similar fashion as regular regression is done, for instance by means of ordinary least squares (OLS);

- By using the decomposition of Sobol' (Sobol' 2001), which does not involve the use of a regression model, but estimates the contributions of different combinations of parameters to the output variance while making the assumption that all parameters are independent. 
Based on the decomposition, sensitivity measures are defined that express the explained variance for different combinations of parameters. The two most commonly reported measures are the first and total-order indices (Saltelli et al. 2008). The firstorder index of a parameter is defined as the reduction of the model variance that would occur, on average, if the parameter became exactly known. The total-order index is defined as the proportion of the variance that would remain, on average, when all other parameters are exactly known. If the input distributions of the parameters are independent, the difference between the first- and total-order index of a parameter measures the explained variance by all of the interactions with other parameters.

\section{Regression-based}

Regression-based methods decompose the variance of the ABM outcomes by fitting a regression function of the input parameters to these outcomes (e.g. Downing et al. (1985)). An advantage of such an approach is that the output of the complex ABM is condensed into descriptive relationships, which can be validated against data using common statistical measures (such as $R^{2}$ ) and which help thinking about influential processes.

However, these relationships are good descriptions of the ABM output only if the regression function is a good fit to the output. If the fit does not explain most of the variance (e.g. $>90 \%$ ) then other regression functions may be tried until a better fit is obtained. Finding a good regression function is thus an iterative process and is a main challenge for regression-based sensitivity analysis, especially for models with complex relations between the input and output.

Various regression methods are available (Draper and Smith 2014). Here we use ordinary least squares (OLS) regression because this method is the most commonly used. It optimises the $R^{2}$ measure for linear regression,

$$
R^{2}=1-\frac{\sum\left(y_{j}-y_{j}^{r}\right)^{2}}{\sum\left(y_{j}-y_{a} v\right)^{2}} .
$$

The summation runs over a number of sample points with different parameter values, $y_{j}$ is the vector of model outcomes, $y_{j}^{r}$ is vector of predicted outcomes by the regression model and $y_{a v}$ is the mean of the model outcomes. The value of $R^{2}$ is interpreted as the explained variance by the regression model. Thus, OLS straightforwardly yields a variance decomposition. The first-order sensitivity index of parameter $i$ is computed as the value of $R^{2}$ when excluding that parameter from the fit,

$$
S_{r, i}=1-\frac{\sum\left(y_{j}-y_{j}^{r}\right)^{2}}{\sum\left(y_{j}-y_{a} v\right)^{2}} .
$$

where $y_{j}^{r}$ is the predicted outcome by the regression function excluding parameter $i$. Similarly, the total-order sensitivity index is computed as the explained variance when keeping parameter $i$, but leaving out all other parameters,

$$
S_{t o t, r, i}=1-\frac{\sum\left(y_{j}-y_{j}^{r}\right)^{2}}{\sum\left(y_{j}-y_{a} v\right)^{2}} .
$$

where $y_{j}^{r^{\prime}}$ is the predicted outcome by the regression function including only parameter $i$. 


\section{Sobol' method}

In contrast to regression-based methods, the Sobol' method is model-free, i.e. no fitting functions are used to decompose the output variance (Saltelli et al. 2008). The method is based on a decomposition of the model variance $V(y)$ that holds if the input parameters are independent,

$$
V(Y)=\sum_{i} V_{i}+\sum_{i, j} V_{i, j}+\ldots+V_{i, j, \ldots, m}
$$

where the partial variance is defined as

$$
V_{i}=V_{x_{i}}\left(E_{x_{i}(y \mid x)}\right)
$$

with $x_{i}$ denoting all parameters except for $x_{i}$. The expectation value of the model output is computed while keeping the parameter fixed and varying all other parameters. The variance of this expectation value is then computed over the possible values of $x_{i}$. Thus, if $V_{i}$ is large, the expected model outcome strongly varies depending on $x_{i}$, indicating the parameter to be sensitive. Sensitivity indices are defined by considering the partial variance relative to the total variance,

$$
S_{s, i}=\frac{V_{i}}{V(y)} .
$$

Higher-order sensitivity indices are defined by computing the partial variance over two or more parameters instead of a single parameter. The total and partial variances are usually estimated from a Monte Carlo sample.

The estimated sensitivity indices by themselves are not meaningful unless we know the accuracy of the estimate. We therefore use a bootstrap method (Archer et al. 1997) to assess this accuracy. We create a number of bootstrap samples by drawing independent points from the original Monte Carlo sample. Each bootstrap sample is equal in size to the original Monte Carlo sample. We then compute the first- and total-order sensitivity indices based on each bootstrap sample. The distribution of these sensitivity indices estimates the accuracy of the sensitivity indices based on the original sample. If the bootstrap sensitivity indices are normally distributed, then we can construct a confidence interval based on the standard error of the bootstrap sensitivity indices.

\subsection{Model test case: agents harvesting a diffusing renewable resource}

The case study concerns an ABM coded in NetLogo (Wilensky 1999) that describes individual agents in a spatially explicit environment. The model flowchart for a single time-step is shown in Fig 4.1. The environment is updated by growth and diffusion of a renewable resource ('food'). The agents observe their local surroundings and decide whether to harvest or not and to move or not, based on their own (decreasing) internal energy state that needs to be replenished, as well as the state of the outside world, which includes the local presence of resource ('food availability') and other agents 
('crowding'). More energy is spent on harvesting or moving than on staying inactive (in which case only basic maintenance processes consume energy). Eventually harvests have to be made in order for agents to survive and reproduce. Upon reproduction, agents pass on individual agent characteristics $w_{\text {harvest }}$ and $w_{\text {move }}$ to their offspring with a small random deviation. These characteristics affect the probabilities that an agent will harvest or move respectively. A detailed description of the model in ODD format is given in Appendix 4.A. The model parameters are listed in Table 4.1. For the purpose of sensitivity analysis, we consider the number of agents $n$ as the output of interest.

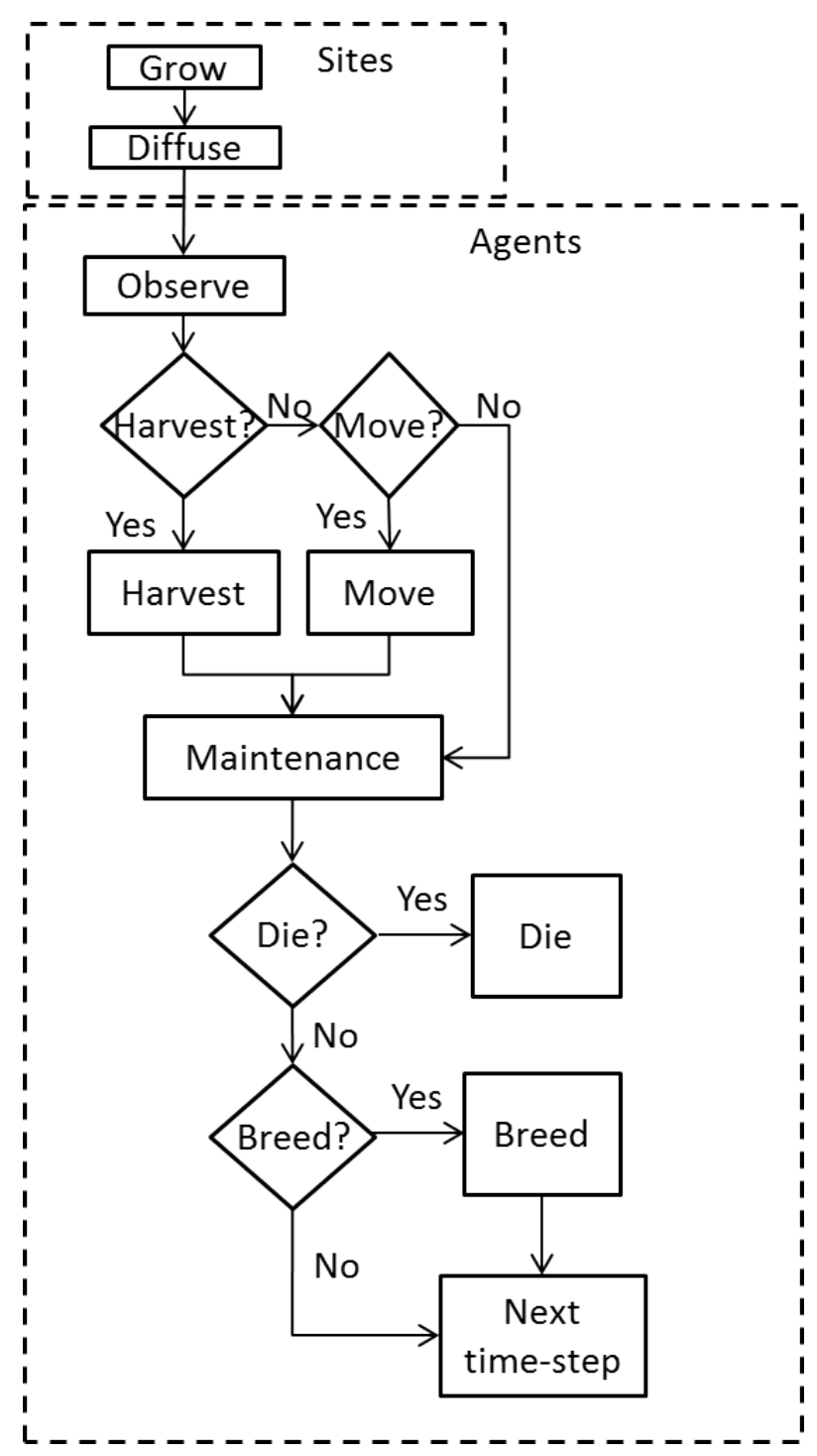

Figure 4.1: Model flowchart for a single time-step 


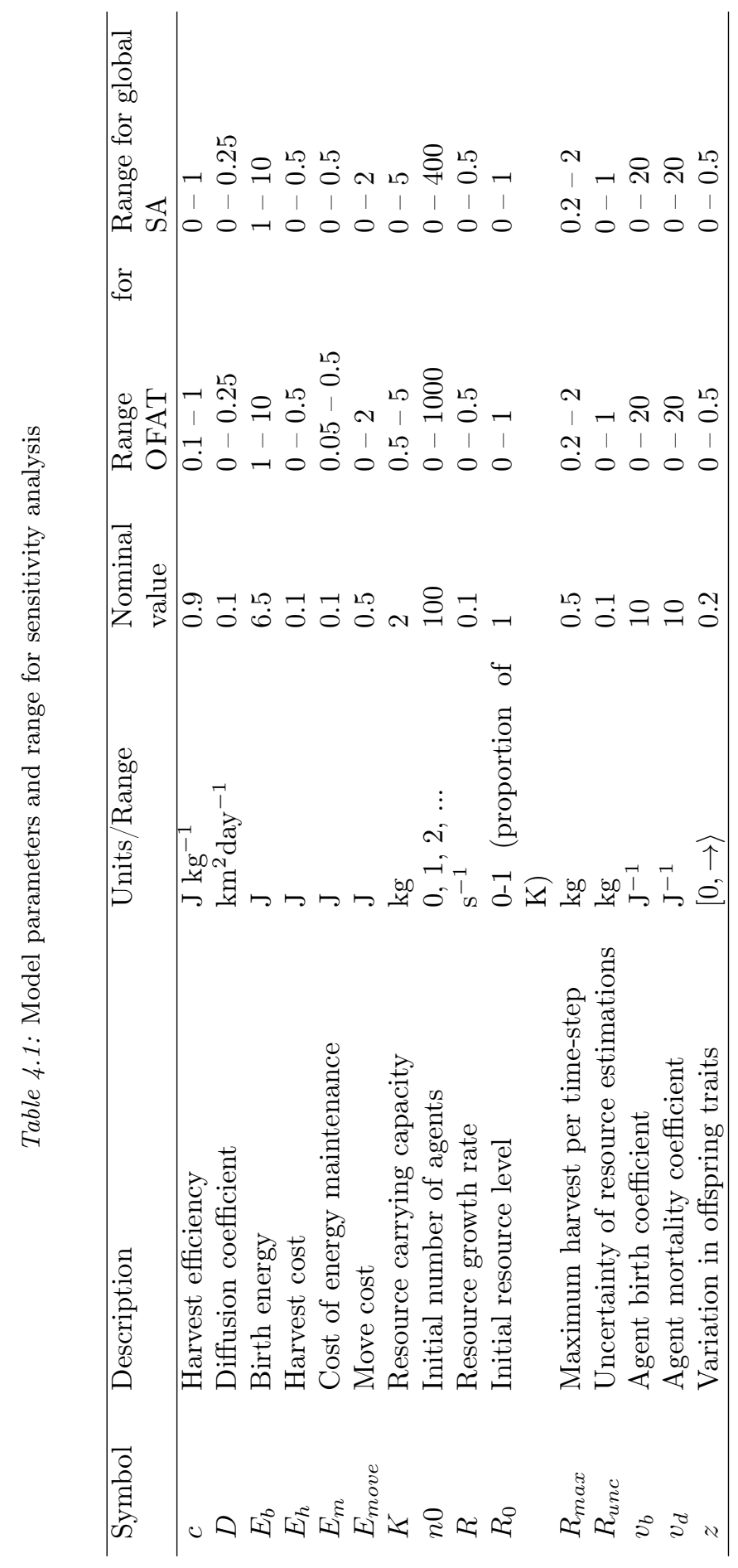




\subsubsection{Choice of inputs and output}

As output of interest for the sensitivity analysis we choose the number of agents $n$. In general it is possible to consider any number of outputs that are of interest. The methods that we discuss here, however, consider only a single output at a time. Thus, the analysis is then performed separately for each output.

We include all of the model parameters and initial conditions in Table 1 as inputs for the sensitivity analysis. For those parameters that are limited to a set range we sample uniformly from this full range. For parameters that do not have a set range we choose a wide range. By testing all parameters uniformly over wide input ranges, we explore the model behaviour without imposing strong assumptions on the input parameters. We thus ensure that we do not ignore interesting model behaviour because it falls outside of the chosen input range. Since we do not a priori know how the model responds to changes in any of the parameters, it is recommended to include all model parameters in the sensitivity analysis. The initial analysis can always be supplemented with more detailed investigations of selected parameters or input ranges. The computational costs, however, can be a limiting factor to the inclusion of model parameters in the sensitivity analysis. The costs for OFAT are lower than for global sensitivity analysis, so an OFAT analysis can be used to select parameters of interest for global sensitivity analysis. Specific interests or hypotheses of the researcher can also guide the choice of inputs.

\subsection{Model results}

\subsubsection{Operational details}

The whole analysis was carried out in R (R Core Team (2012)). The sample points for the sensitivity analysis were also generated in $\mathrm{R}$. The sample points were exported to perform the model runs in Netlogo.

We conduct a pre-test to determine whether the model converges to a steady state behaviour. The pre-test consists of 1000 model runs at parameter values drawn from uniform distributions within the range for global sensitivity analysis listed in Table 1. We record the output at every time-step. The output initially changes strongly in time, but from $t=500$ to $t=1000$ it seems to have stabilised and consist of random fluctuations around a mean (Fig 4.2). We conclude that 500 time-steps is a reasonable time period for the sensitivity analysis, since the model output does not strongly change between $t=500$ to $t=1000$. There are, however, random fluctuations around the mean. Furthermore, runs at the nominal set show that the number of agents oscillates in time. To prevent the output from being strongly dependent on the random fluctuations and the phase of the oscillations, we average $n$ from $t=500$ to $t=1000$.

Even though the output does not change strongly between $t=500$ to $t=1000$, there seems to be a small and gradual increase in the mean value. This increase is caused by a gradual shift in the distribution of the agent characteristics $w_{\text {harvest }}$ and $w_{\text {move }}$, which affect the probabilities of harvesting or moving. This shift results from the inheritance of these characteristics during reproduction. On average, values that increase the chances of an agent to survive and reproduce are passed on more 


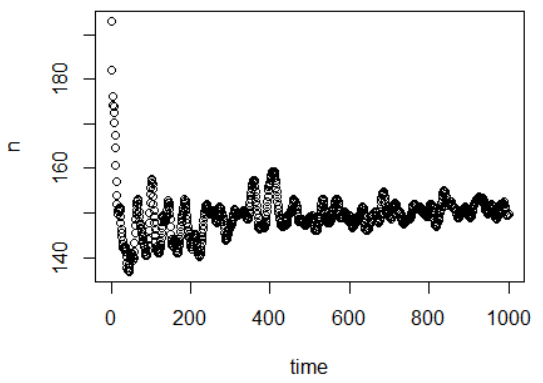

Figure 4.2: Plotted is the average $n$ for 1000 model runs with parameter values randomly drawn from uniform distributions in the range for global sensitivity analysis listed in Table 4.1. There are strong changes in $\mathrm{n}$ over the first few hundred time-steps. From $t=500$ to $t=1000, n$ seems to have converged to random fluctuations around a mean.

frequently. On the long-term, this causes a change in the distributions of those characteristics and this affects the number of agents supported by the environment. For the sensitivity analysis, however, we do not examine this long-term adaptivity. The chosen time-scale from $t=500$ to $t=1000$ is sufficiently short not to be strongly affected by the adaptivity, and sufficiently long not to depend strongly on random fluctuations and oscillations.

We perform 10,000 replicate runs in the default parameter set to estimate the distribution of the output. The stabilization of the coefficient of variation,

$$
c_{v}=\frac{\sigma(n)}{\mu(n)},
$$

where $\sigma(n)$ is the standard deviation of the output and $\mu(n)$ is the mean of the output, indicates the required number of replicates to obtain a proper estimate of the distribution of the model output (Lorscheid et al. (2012)). Fig 4.3 shows that the coefficient of variation is largely stabilised after a few hundred replicates. The form of the distribution of $n$ depends strongly on the parameter values. In the nominal set, the distribution over 10,000 replicates (Fig 4.4a) has a heavy tail on the right side. At $D=0.05$, however, the distribution resembles a normal distribution (Fig 4.4b), whereas at $D=0.15$ it shows a large peak at $n=0$ (Fig 4.4c). This peak is caused by extinction of the agent population that occurs stochastically in a region of the parameter space. We will return to this finding when we discuss the results of the OFAT sensitivity analysis.

For the OFAT sensitivity analysis, we use 10 replicates per parameter setting in order to roughly estimate the spread of the output. For the regression-based sensitivity analysis, 5 replicates are used in order to estimate the proportion of the variance of the output that is explained by variations in the input parameters. For the Sobol' method, we follow the usual procedure of sampling as many different points as possible to accurately estimate the sensitivity indices (Saltelli et al. 2008). We 


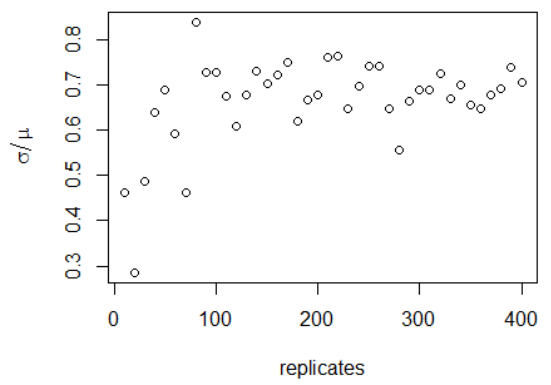

Figure 4.3: Coefficient of variation of the average number of agents for increasing number of replicates in the nominal parameter set. The coefficient of variation has largely stabilised after a few hundred model runs, indicating this number is sufficient for estimation of the distribution of $n$.

therefore do not use any replicates. A number of sampling designs are available to minimise the computational costs of the estimation (e.g. Cukier et al. (1978), Tarantola et al. (2006), Saltelli (2002)). Here we use the design by (Saltelli 2002), with a total sample size of 17,000 model runs. This design is relatively easy to use and it estimates all of the first- and total-order sensitivity indices. A more detailed description of the design is given in Appendix 4.C.

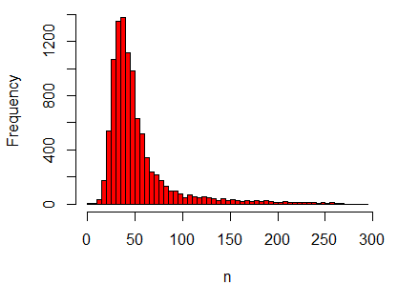

(a)

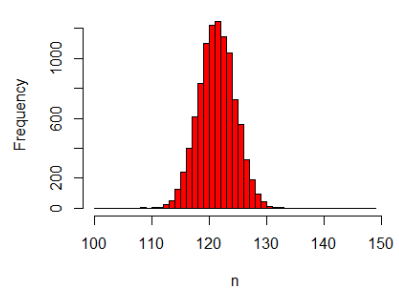

(b)

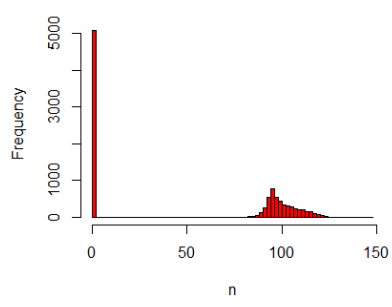

(c)

Figure 4.4: (a): Histogram of 10,000 samples in the nominal set $(D=0.1)$ displaying the average number of agents between $t=500$ and $t=1000$. The distribution has a long tail for large values of $n$. (b): Same as (a), but for $D=0.05$. The resulting distribution resembles a normal distribution. (c): Same as (a), but for $D=0.15$. The resulting distribution is split between a peak at $n=0$ and a distribution around $n=100$. The peak is caused by stochastic extinction that occurs for some values of $D$ (see OFAT results) 


\subsubsection{One-factor-at-a-time sensitivity analysis}

We perform OFAT sensitivity analysis for all of the model parameters. The default values and ranges of variation of all parameters are listed in Table 4.1. We run the model for the extreme values of this range and 9 equidistant points in between. The resulting graph for the diffusivity (Fig 4.5) shows two tipping points where the number of agents goes to zero. When $D=0$, sites without resource cannot be replenished through diffusion from neighbouring sites, eventually leading to collapse of the system. Inspection of the time-series of a number of model runs reveals that the number of agents oscillates with increasing amplitude for increasing values of $D$. Between $D=0.10$ and $D=0.15$ this amplitude becomes so large that n crosses zero, causing the system to collapse. Because the model is stochastic, this collapse occurs in some but not all of the replicates. Tests with longer simulation times than 1000 time-steps show that between $D=0.10$ and $D=0.15$ extinction occurs mostly during the first 1000 time-steps. If extinction does not occur during this initial period, then $\mathrm{n}$ remains positive on the long term. The model thus has multiple equilibria for these parameter values. For $D \leq 0.175$ the population always goes to extinction, but for $D=0.175$ it sometimes takes more than 1000 time-steps before this equilibrium is reached. One of the model runs in Fig 4.5 therefore still has a positive population after 1000 time-steps.

The graphs for the other parameters are shown in Appendix 4.B. Out of a total of 15 model parameters, 11 parameters show a tipping point where the number of agents goes to zero. Furthermore, most parameters show a nonlinear response when the number of agents is positive. The graphs yield mechanistic understanding of the model. We pick a few parameters for a brief discussion. For higher values of $r$, the resource replenishes faster and the system supports a larger number of agents. The carrying capacity $K$ also has a positive effect on the number of agents, but higher values destabilise the system and cause collapse of the population. Low values for the energy costs required for maintenance $E_{m}$ and for harvesting $E_{\text {harvest }}$ also lead to collapse by causing an increase in the amplitude of the oscillations of the number of agents. High values of $E_{m}$ and $E_{\text {harvest }}$ lead to lower agent numbers because the system is able to support fewer agents if those agents require more energy. A high energy cost for moving $E_{\text {move }}$, however, does not lead to low agent numbers. For high move costs, the agents will simply move less often and instead decide to harvest at their current position. The initial conditions $n_{0}$ and $R_{0}$ can affect the final state of the system by causing an early collapse, but have no effect on the final state if the number of agents remains positive.

\subsubsection{Regression-based sensitivity analysis}

The regression-based global sensitivity analysis is based on $N_{r}=1000$ sample points drawn from uniform probability distributions over the range given in Table 4.1. The range for the initial number of agents for global sensitivity analysis is smaller than for OFAT. Using the same range as the OFAT, the number of agents reaches zero in almost $90 \%$ of the model runs. Extinction of the agent population is an important model outcome and we are therefore interested in understanding the conditions under which extinction takes place. However, to assess the parameter sensitivity indices we need a sufficient number of runs with nonzero outcome. We therefore reduce the range 


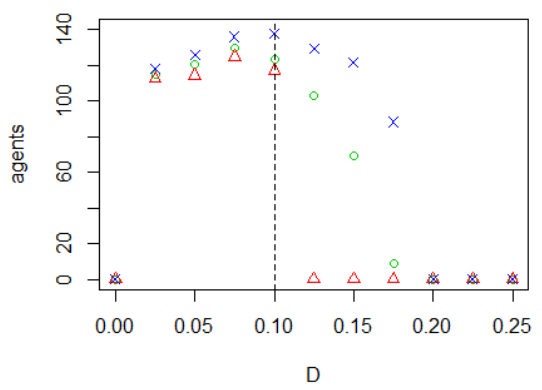

Figure 4.5: Results of the OFAT analysis for D. Displayed are the mean (green circle), minimum (red triangle) and maximum (blue cross) of the number of agents among 10 replicates. The dashed line represents the nominal value.

for $n_{0}$ for the global sensitivity analysis. The reduced range has a larger number of runs with positive outcomes, but also contains runs where the population goes extinct.

For each sample point, we use 5 replicate runs to estimate the variation of the model output due to stochasticity. To limit the computational expense this number of replicates is smaller than the 10 replicates for the OFAT. The mean output variance over replicates is $0.88 \%$ of the output variance over all samples. The variance due to stochasticity is thus negligible compared to the variance due to parameter variations and unexplained variance cannot be attributed to the stochasticity inherent in the model. Since the variance due to stochasticity is so small, we do not need a more accurate estimate of this variance, which gives us confidence that we do not need to increase the number of replicate runs per parameter setting.

We fit a first-degree polynomial (Eq 4.8) of the model parameters $x_{i}$ to the output using ordinary least squares regression (OLS),

$$
n=b_{0}+\sum_{i}^{m} b_{i} x_{i} .
$$

The outcomes show that the total number of 5000 model runs was sufficient to obtain reasonably accurate estimates of the explained variance and the first-order sensitivity indices (Table 4.2). However, the coefficient of determination $R^{2}=43.9 \pm 4.1 \%$ (Table 4.3) shows that more than half of the model variance is unexplained. We attempt to increase the explained variance by adding higher order terms to Eq 4.8. We distinguish between terms that are nonlinear in a single parameter $x_{i} x_{i}$ and interaction terms $x_{\sim i} x_{j}$. Table 4.3 lists the values of $R^{2}$ for a number of functions that are obtained by adding such terms. The increase in explained variance should be weighed against the increase in the complexity of the regression model. Functions with a large number of terms will generally yield high values of $R^{2}$ simply due to the larger number of degrees of freedom. Table 4.3 shows that even with a very large number of terms a large part of the model variance still remains unaccounted for. Furthermore, such a large number of terms is not useful in terms of gaining insight into the influential model processes. A common alternative to fitting a first-degree polynomial (Eq 4.8) 
is fitting the logarithm of the parameter instead of the parameter itself. Doing this for some of the model parameters increases the value of $R^{2}\left(R^{2}=48.1 \%\right)$. However, the increase is only small compared to a polynomial fit and still leaves a large part of the variance unexplained.

Figs $4.4 \mathrm{a}-4.4 \mathrm{c}$ show that the form of distribution of $n$ depends on the parameter values. The distribution over the $N_{r}=1000$ samples used for the regression-based sensitivity analysis (Fig 4.6) contains a large peak at $n=0$ and a long tail with a number of outliers for large values of $n$. For these kind of asymmetric distributions, OLS, which assumes normally distributed residuals, does not generally produce good fits (Draper and Smith 2014). Specifically, outlying values relative to the normal distribution tend to destabilize the regression function. The explained variance $R^{2}$ is then no longer a good measure. This presents a possible explanation for the failure to obtain a good fit. Moreover, the outliers that destabilize the OLS regression weigh heavily in the variance itself. This is illustrated by Fig 4.7, which visualizes the contributions of different sample points to the variance. On the horizontal axis, the sample points are sorted by increasing value of the output $n$. For each point we plot the partial sum of the squared deviations from the mean, where the mean is computed over all sample points. The slope of the graph in a point measures the contribution of that sample point to the variance. The slope is initially straight, because a large number of sample points with outcome $n=0$ contribute equally to the variance. Each coloured area corresponds to $25 \%$ of the total variance. Outlying values represent a large amount of the total variance. Out of $N_{r}=1000$ samples, the 14 highest values represent $25 \%$ of the variance, and the 61 highest values represent $50 \%$ of the variance. The dashed line represents the explained variance by the regression model (Eq 4.8). The regression model does not adequately capture the contribution of outliers to the variance. The outliers give valuable information on the extreme cases of model behaviour and the conditions under which such extreme behaviour occurs. It is therefore important to consider these outliers as part of sensitivity analysis. However, since these outliers represent a major part of the variance, any sensitivity analysis method based on variance decomposition will assign them a disproportionate weight.

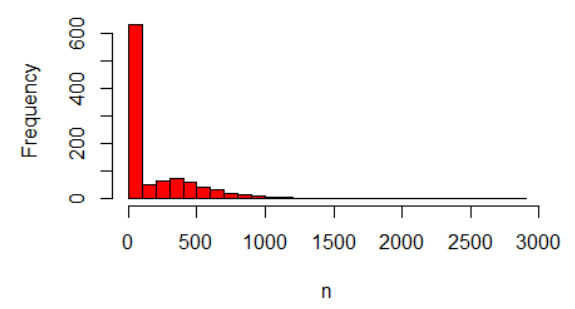

Figure 4.6: Histogram of the number of agents, for the $N_{r}=1000$ sample points used for the regression-based sensitivity analysis. The histogram shows a large peak at $n=0$ and contains a number of outliers between $n=1000$ and $n=3000$. 
Table 4.2: First-order indices according to Eq 4.8. The confidence intervals are based on a bootstrap sample of size 10,000. Only parameters with at least $1.0 \%$ explained variance are included. We also report the standardized regression coefficients $\beta_{i}=b_{i} \frac{\sigma(n)}{\sigma\left(x_{i}\right)}$. Here the standard deviation $\sigma(n)$ is computed from the output sample of the sensitivity analysis and the standard deviation $\sigma\left(x_{i}\right)$ is given by the input distribution of the parameter $x_{i}$ (see e.g. Cariboni et al. (2007)). The standardized regression coefficients measure the strength as well as the direction of the effect of the parameter on the output.

\begin{tabular}{lllllllll}
\hline Par. & $k$ & $r$ & $E_{m}$ & $R_{\max }$ & $E_{h}$ & $c$ & $D$ & Total \\
$S_{r}$ & $20.0 \%$ & $9.1 \%$ & $5.7 \%$ & $4.1 \%$ & $3.3 \%$ & $1.6 \%$ & $1.0 \%$ & $45.5 \%$ \\
& \pm & \pm & \pm & \pm & \pm & \pm & \pm & \pm \\
& $3.8 \%$ & $3.0 \%$ & $2.6 \%$ & $2.2 \%$ & $1.9 \%$ & $1.4 \%$ & $1.2 \%$ & $4.1 \%$ \\
$\beta$ & 0.43 & 0.29 & -0.24 & 0.23 & -0.18 & 0.23 & 0.10 & - \\
\hline
\end{tabular}

Table 4.3: $R^{2}$ for various polynomial regression models with increasing number of terms. A significant increase in the explained variance is obtained by adding terms to the regression model, but this should be weighed against the increased complexity of the regression model. The table shows a large number of terms is needed to significantly increase the explained variance.

\begin{tabular}{|c|c|c|c|c|c|c|c|c|}
\hline $\begin{array}{l}\text { order of } \\
\text { nonlinearities }\end{array}$ & $1 \mathrm{st}$ & 2nd & $3 \mathrm{rd}$ & $1 \mathrm{st}$ & 2nd & $1 \mathrm{st}$ & 2nd & $3 \mathrm{rd}$ \\
\hline interactions & - & - & - & 2nd & 2nd & $3 \mathrm{rd}$ & $3 \mathrm{rd}$ & $3 \mathrm{rd}$ \\
\hline coefficients & 16 & 31 & 46 & 121 & 136 & 576 & 591 & 606 \\
\hline$R^{2}$ & $45.5 \%$ & $49.9 \%$ & $51.2 \%$ & $63.7 \%$ & $67.7 \%$ & $65.5 \%$ & $69.0 \%$ & $70.0 \%$ \\
\hline
\end{tabular}




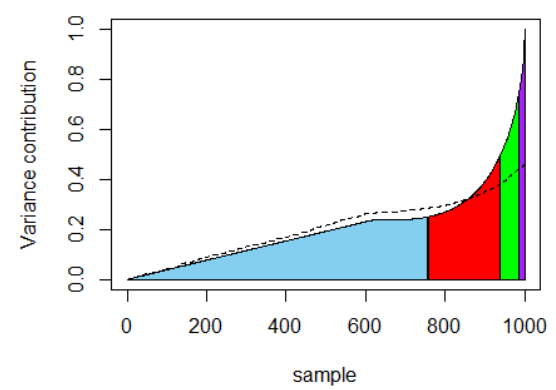

Figure 4.7: Contribution to the variance of each sample for the regression-based sensitivity analysis. The sample points are sorted by increasing output $n$. The variance contributions are then computed as the partial sum of squared deviations from the mean, assuming the mean to be constant. The values are normalised by the total variance. Each colour represents $25 \%$ of the total variance. For the dashed line, the output for each sample is replaced by the predicted output of the regression model (Eq 4.8). A small number of outliers on the right contributes strongly to the total variance, but this contribution is not adequately captured by the regression model.

\subsubsection{Sobol' method}

The computation of the Sobol' main and total-order sensitivity indices was carried out using the sampling scheme by Saltelli (2002) with a total sample size of 17,000 model runs. We used uniform distributions in the range given in Table 4.1. The resulting first- and total-order indices are shown in Fig 4.8. We assess the accuracy of the estimated sensitivities using bootstrap confidence intervals, based on 10,000 bootstrap samples (Archer et al. 1997). Some of the intervals are wide, showing that the estimate is not accurate. Furthermore, a number of the confidence intervals contain negative values. These negative ranges have no valid interpretation because the sensitivity represents a portion of the model variance, which is bound between $0 \%$ and $100 \%$. The sensitivity estimates, however, can be negative due to numerical inaccuracy of the method (Saltelli et al. 2008). Thiele et al. (2014) report the same finding, but discard the negative ranges by setting them to zero. However, this introduces a bias in the bootstrap confidence interval. For example, in our results the sensitivity of the parameter $E_{b}$ has a wide confidence interval that spans mostly negative values. If we discard these negative values, then the sensitivity would appear to be estimated at zero with a very high precision. We therefore report the negative values. Appendix 4.C provides a more detailed explanation of these negative outcomes.

The sum of all first-order indices equals 0.46 . This includes both linear and nonlinear contributions of individual parameters, but no interaction effects. More than half of the model variance is thus explained by interaction effects according to the variance decomposition of Eq 4.4. The total-order indices show that a number of parameters explain little variance even including interaction effects. Additionally, the more influential parameters have wide confidence intervals for the total-order sensitiv- 
ities. We thus have only rough estimates of the amount of variance those parameters can explain including their interactions. However, a few parameters, such as $K$ and $R_{\max }$ are clearly influential, because even the lower ends of the confidence intervals of the total-order sensitivities are large compared to the sensitivity estimates of other parameters.

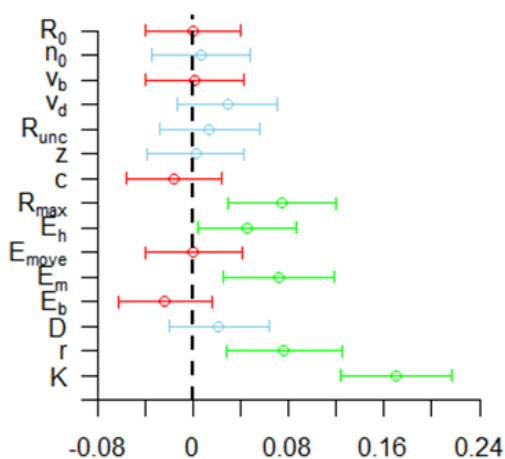

(a)

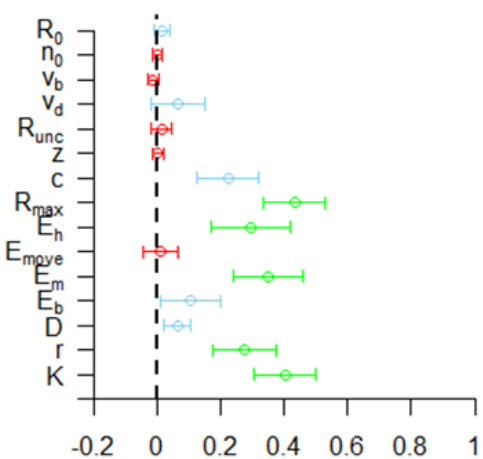

$\mathrm{S}_{\text {tot }}$

(b)

Figure 4.8: First-order (a) and total-order (b) Sobol' indices for all parameters. The colours categorize the parameters from most influential (green) to averagely influential (blue) and least influential (red).

\subsection{Discussion}

We have applied three commonly employed methods of sensitivity analysis to a typical but low-complexity ABM. Here we evaluate each method in terms of our three selected aims (1/ understanding the emergence of patterns, 2 / evaluating robustness, and 3 / evaluating outcome uncertainty). Table 4.4 summarizes the outcomes of this evaluation. We will discuss the Table in more detail below.

The OFAT method yields insight into qualitative aspects of model behaviour and the patterns that emerge from the model. It gives important information about the functional form of the relationships between parameters and output variables. Furthermore, it shows the existence of tipping points and other strong nonlinearities. Exposing such qualitative aspects can lead to a better mechanistic understanding of the behaviour of the model and thus the modelled system. OFAT can also be used to show the robustness of model behaviour with respect to changes in single parameters, thus revealing whether emergent patterns depend on strong assumptions. Interaction effects are not considered, and therefore OFAT is not suitable for further quantification of sensitivity indices. OFAT should therefore be supplemented by some global method of sensitivity analysis to examine interaction effects and to quantify model variability that results from parameter variations. For our case, for example, global sampling from the parameter space revealed that interaction effects lead to the existence of outliers for which the output takes extreme values. Since each parameter 
is only varied individually and not in combination with other parameters, OFAT is relatively cheap in terms of computational costs. At 10 replicates for 11 parameter values, we need 110 model runs per parameter, or 1650 runs in total. This cost could still be lowered by decreasing the number of replicates.

Regression-based sensitivity analysis is a global method based on variance decomposition. A major limitation for application to sensitivity analysis of ABMs is that it depends on the structure of the model whether a good decomposition can be obtained by regression. In our case a good fit (with $R^{2}>0.9$ ) was not obtained even using a third-order polynomial function because of the existence of outlying values as well as regions of parameter space where the output goes to zero. This corresponds to previous reporting difficulties by Burgers et al. (2010) on finding a well-fitted function for an ABM of trading agents. They attributed these difficulties to the inherent complexity of the model. We agree with this assessment, where we explicitly add that in our case the lack of fit is better understood by considering the results we obtained by first performing the OFAT. The lack of fit resulting from the inherent complexity of ABMs limits the usefulness of regression-based sensitivity analysis for these models, as the systems which they are used to model are complex adaptive systems that are characterized by the existence of nonlinear interactions and feedbacks, multiple levels of model behaviour, and emergent properties. These characteristics complicate and obscure the relations between the model inputs and outputs and are thus not straightforwardly captured by a regression function. Even though the regression function does not yield a good fit, however, regression-based sensitivity analysis might prove useful in selecting influential parameters for further analysis based on the sensitivity indices or regression coefficients. The computational costs of regression-based sensitivity analysis lie somewhere in between OFAT and the Sobol' method. For our test-case 1000 parameter settings with 5 replicates each were sufficient to obtain reasonably accurate estimates of the first-order indices.

The Sobol' method does not use fitted functions, but instead decomposes the variance based directly on a sample from the parameter space (Saltelli et al. 2008). The method aims to quantify the output variability based on this variance decomposition. Whereas the variance has a clear interpretation for normal distributions, it is not a good measure of variability for distributions that are skewed or contain outliers (Swinscow 1997). This problem is illustrated by Fig 4.7, which shows that the output variance of our test case is inflated by a few outlying sample points. The method is therefore not suitable for quantifying outcome variability of ABMs with output distributions that have outliers, are skewed, or strongly deviate in other ways from the normal distribution (Liu et al. 2006). Another drawback of the method is that the method does not allow for the possibility to distinguish between mechanistic explanations on why a parameter is influential. For example, in our test case the harvesting cost shows up as an influential parameter (Fig 4.8b), but it is unclear whether it has some effect on outlying values, or causes collapse of the population, or affects the number of agents for positive population sizes. In contrast, the OFAT results show that it causes population collapse and affects the number of agents for positive populations, but such specific findings are lost in the Sobol' method because sensitivities are aggregated over the full parameter space (Rakovec et al. 2014). As such, the method also does not reveal the robustness of model patterns to parameter changes within this space. Out of the methods we tested, the Sobol' method has the 
largest computational costs. With a total of 17.000 model runs, the estimates of the sensitivity indices have wide confidence intervals. For a number of parameters these intervals included negative values, which have no valid interpretation.

In light of these results we recommend OFAT as the starting point for any sensitivity analysis of an ABM, in particular when one wants to gain insight into the mechanisms and patterns that ABMs produce, which is a typical goal of many social simulation studies. This recommendation seemingly contradicts the one by Saltelli et al. (2008) and Ligmann-Zielinska et al. (2014), who suggest the use of the Sobol' method for model analysis. However, our study suggests that standard methods of global sensitivity analysis do not adequately address the issues that are relevant for the analysis of ABMs, such as the existence of multiple levels, nonlinear interactions and feedbacks, and emergent properties, as effectively as OFAT. Obviously any OFAT can be supplemented with a global method to investigate interaction effects, given that certain conditions are met, namely that the output distribution is and remains sufficiently similar to a normal distribution, regression fits have a high $R^{2}$, and confidence intervals have positive bounds. Some promising advancements in other fields might prove useful for ABM applications. These include a hybrid local-global sensitivity method that examines the distribution of explained variances over portions of the parameter space (Rakovec et al. 2014) and alternative sensitivity measures based on entropy instead of variance (Liu et al. 2006, Auder and Iooss 2008). 


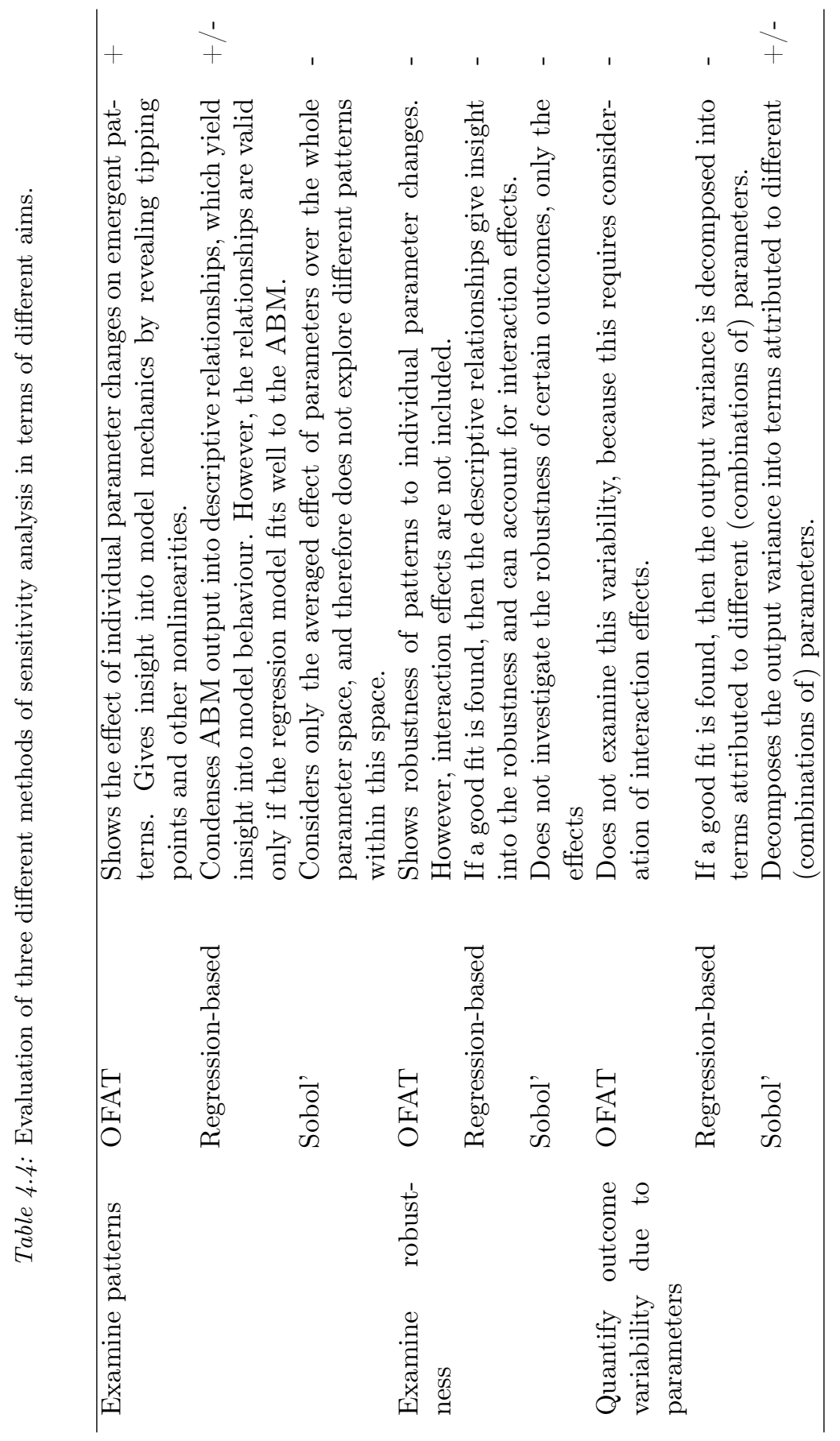


Table 4.5: State variables. The upper part of the table contains the site state variables. The lower part contains the agent state variables.

\begin{tabular}{llll}
\hline Symbol & Description & Dimensions & Range \\
$i, j$ & Position indices & - & $0,1, \ldots, \mathrm{L} ; 0,1, \ldots, \mathrm{L}$ \\
$R_{i, j}$ & Resource & $\mathrm{kg}$ & {$[0, \rightarrow\rangle$} \\
$R_{i, j}^{a}$ & Estimated resource & $\mathrm{kg}$ & {$[0, \rightarrow\rangle$} \\
$x_{a}$ & Position & - & $(0,1, \ldots, \mathrm{L} ; 0,1, \ldots, \mathrm{L})$ \\
$a$ & Agent index & - & $0,1,2, \ldots$ \\
$w_{h}^{a}$ & Harvest coefficient & - & {$[0, \rightarrow\rangle$} \\
$w_{m}^{a}$ & Move coefficient & - & {$[0, \rightarrow\rangle$} \\
\hline
\end{tabular}

\section{A Model description}

The model description follows the ODD (Overview, Design concepts, Details) protocol for describing individual- and agent-based models (Grimm et al. 2006, 2010).

\section{A.1 Purpose}

The basic topic of the model is how individual agents in a spatial environment make trade-offs between conserving energy by remaining inactive and becoming active to gather energy. Energy is gathered from the environment and spent on a daily maintenance that represents the physiological processes of the agent and on the actions of moving and harvesting.

\section{A.2 Entities, state variables, and scales}

The state variables are listed in Table 4.5. The landscape is represented by a square lattice of length $L$. Each site $i, j(i, j=1,2, \ldots, L)$ has a length of $\Delta x=1 \mathrm{~km}$. Since the model was not fitted to realistic data, this choice is to some extent arbitrary. Setting $\Delta x=1$, is convenient for computations with parameters that have length-units in their dimensions. Periodic boundary conditions are imposed, so that $i+L=i$ and $j+L=j$. Each site has a resource level $R_{i, j}$.

Agents move over the lattice and harvest resource. An agent is identified by the index $a=1,2, \ldots$. Each agents has an energy level $E_{a}$ and a location $x_{a}$. Agents estimate the resource levels at their site and at the four Von Neumann nearest neighbour sites every time-step. Thus, an agent with location $x_{a}=i, j$ has estimates for $R_{i, j}, R_{i \pm 1, j}$ and $R_{i, j \pm 1}$. Agents also have harvest and move coefficients, $w_{h}^{a}$ and $w_{m}^{a}$. Agents with a high value for $w_{h}^{a}$ or $w_{m}^{a}$ are less likely to harvest resource or move to a neighbouring site respectively. The values of $w_{h}^{a}$ and $w_{m}^{a}$ are constant for each agent.

Time is modelled in discrete time steps of $\Delta t=1$ day. Like the distance scale of $\Delta x=1$, this was chosen to make computations convenient. 


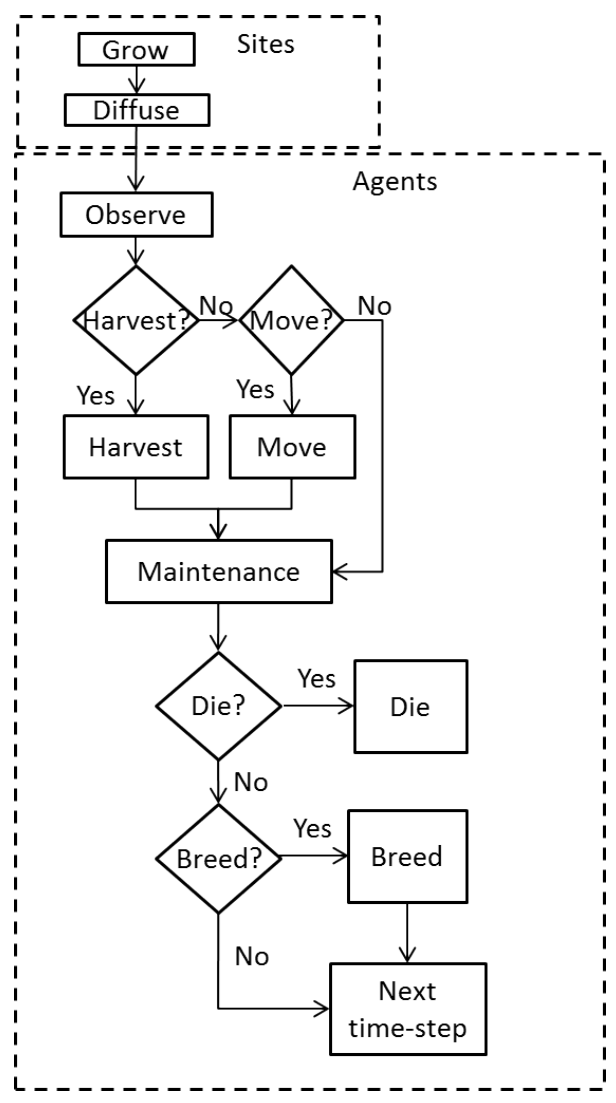

Figure 4.9: Flowchart for a single time-step.

\section{A.3 Process overview and scheduling}

The model flow chart for a single time-step is shown in Fig 4.9. The submodels in the boxes are described in detail in the submodel section. Each submodel is run for all of the patches/agents before the start of the next submodel.

The time-step starts with the updating of the sites. The 'grow' submodel grows resource on each site following a logistic growth equation. The 'diffuse' submodel diffuses the resource between sites following Fick's second law (Fick 1855).

The updating of sites is followed by the agent actions. Each agent first 'observes' the resource level and the number of agents on neighbouring sites. The agents then decide whether to harvest, based on their internal energy state, the estimated resource level on their location, and the presence of other agents. If an agent does not harvest, it may decide to move based on the number of agents and estimated resource level on neighbouring sites. Alternatively, an agent may decide to neither harvest nor move, thus conserving energy. After all the agents have decided, the actions of harvesting and moving are carried out in random order. Each agent then pays a fixed energy maintenance. The internal energy state of each agent affects the probabilities of dying and breeding. The model then proceeds to the next time-step. 


\section{A.4 Design concepts}

\section{Basic principles}

The model considers agents that move and harvest in a spatial environment. Based on their internal state and the state of the environment agents may decide to move to a new location, to harvest from the present location, or to wait for a better opportunity. Harvested resource is converted to internal energy, which is used to pay daily maintenance. The energy for maintenance The acts of moving and harvesting also cost energy. The decisions to move and harvest are based on both the internal energy state of the agent and the state of the environment.

\section{Emergence}

We consider the total population size $n_{t o t}$ and the values of the harvest and move coefficients $w_{h}^{a}$ and $w_{m}^{a}$ averaged over the population as emerging variables at the macro-level.

\section{Adaptation}

Agents adapt their behaviour to changes in their internal state or in their environment. Agents with low levels of internal energy tend to harvest resource with a higher probability. Agents also tend to harvest if the resource level on their site is high and the number of other agents on the site is low.

Agents consider the state of the sites in the Von Neumann neighbourhood in their decisions to move to neighbouring sites. Agents are more likely to harvest if the resource level on their current site is high and there are few other agents on this site. Agents are more likely to move to sites with high resource levels and few other agents.

\section{Objectives}

The first objective of each agent is to gather sufficient resource to ensure its own survival. The second objective is to accumulate sufficient energy to breed.

\section{Learning}

Individual agents do not change their adaptive traits over time. However, the distribution of these traits over the population may change due to selection. When agents breed, their offspring inherit the parents' values of $w_{h}^{a}$ and $w_{m}^{a}$ with some random variation. If, for example, agents with a low value $w_{m}^{a}$ reproduce more frequently often on average, then low values will become increasingly common over time.

\section{Prediction}

Agents tend to move less often to sites that are occupied by one or more other agents, based on the prediction that those other agents may harvest resource from the site. Agents tend to harvest more often at their present location if neighbouring locations are occupied by other agents that might move to the resource. Agents also tend to move to sites with high resource levels, predicting that the resource level will remain high and can be harvested. If an agent finds the present conditions unsuitable for 
harvesting, that agent may decide to wait before taking action, expecting that the conditions will improve in the future.

\section{Sensing}

Agents sense their own internal energy. Each time step, agents assess the amount of resource on their current location and the four sites that compose the Von Neumann neighbourhood. However, agents do not sense the exact amount of resource because there is some random error. Agents also sense the presence of any other agents within the Von Neumann neighbourhood, without any error.

\section{Interaction}

Indirect interactions between agents occur through the competition for resource. In addition, agents are less likely to move to or harvest at sites that contain other agents. This is a form of direct interaction. Agents are also more likely to harvest on their present site if neighbours are occupying the neighbouring sites. The basic model formulation does not include further direct interactions between agents in order for the model to function as a benchmark.

\section{Stochasticity}

The decisions of agents to harvest at the present location or to move to a neighbouring site are based on smooth probability functions. The functions take as input the number of agents and the perceived amount of resource within the Von Neumann neighbourhood. The perceived amount of resource differs from the actual amount by an amount that is drawn from a normal distribution. Birth and death of agents are also stochastic, but based on the internal energies of agents. If an agents breeds, the offspring inherit the values of $w_{h}^{a}$ and $w_{m}^{a}$ with a small random variation. Furthermore, the agents' initial locations, energies and values of $w_{h}^{a}$ and $w_{m}^{a}$ are also stochastic.

\section{Observation}

Three output variables are collected: the total number of agents $n$ and the averages of $w_{h}^{a}$ and $w_{m}^{a}$ over all agents. All output variables are recorded at each time-step.

\section{A.5 Initialization}

All sites are initialized with resource levels equal to $R_{0}$ times carrying capacity. The initial number of agents is specified as a parameter, $n_{0}$. Each agent is placed on a random site with an internal energy that is drawn from a uniform distribution between 0 and the minimum energy that is needed for procreation. The initial values of $w_{h}^{a}$ and $w_{m}^{a}$ are drawn from a uniform distribution between 0 and 1 . The default parameter values of the model are listed in Table 4.6.

\section{A.6 Input data}

The model does not use input data to represent time-varying processes. 
Table 4.6: List of used symbols in the model description. The upper part contains general notation, the middle part model variables and the lower part model parameters

\begin{tabular}{|c|c|c|c|}
\hline Symbol & Description & Units/range & $\begin{array}{l}\text { Default } \\
\text { value }\end{array}$ \\
\hline$i, j$ & Site indices & $1,2, \ldots, \mathrm{L}$ & - \\
\hline$L$ & Lattice size & - & 33 \\
\hline$T$ & Time index & day & - \\
\hline$a$ & Agent index & - & - \\
\hline$\Delta t$ & Time-step size & day & 1 \\
\hline$\Delta x$ & Site length & $\mathrm{km}$ & 1 \\
\hline$E_{a}$ & Initial energy & $\mathrm{J}$ & - \\
\hline$N$ & Total number of agents & $0,1,2, \ldots$ & - \\
\hline$n_{i, j}$ & Number of agents on site $i, j$ & $0,1,2, \ldots$ & - \\
\hline$R_{h}$ & Harvested resource & $\mathrm{kg}$ & - \\
\hline$R_{i, j}$ & Resource on site $i, j$ & $\mathrm{~kg}$ & - \\
\hline$R_{i, j}^{a}$ & Observed resource by agent $a$ & $\mathrm{~kg}$ & - \\
\hline$w_{h}^{a}$ & Harvest coefficient & - & - \\
\hline$w_{m}^{a}$ & Move coefficient & - & - \\
\hline$x_{a}$ & Location of agent $a$ & $(0,1, \ldots L ; 0,1, \ldots L)$ & - \\
\hline$c$ & Efficiency & $\mathrm{J} \mathrm{kg}^{-1}$ & 0.9 \\
\hline$D$ & Diffusion coefficient & $\mathrm{km}^{2} \mathrm{day}^{-1}$ & 0.1 \\
\hline$E_{b}$ & Birth energy & $\mathrm{J}$ & 5 \\
\hline$E_{h}$ & Harvest cost & $\mathrm{J}$ & 0.1 \\
\hline$E_{m}$ & Cost of energy maintenance & $\mathrm{J}$ & 0.1 \\
\hline$E_{\text {move }}$ & Move cost & $\mathrm{J}$ & 0.5 \\
\hline$K$ & Carrying capacity & $\mathrm{kg}$ & 2 \\
\hline$n_{0}$ & Initial number of agents & $0,1,2, \ldots$ & 100 \\
\hline$r$ & Growth rate & $\mathrm{s}^{-1}$ & 0.1 \\
\hline$R_{0}$ & Initial resource & $\begin{array}{ll}0-1 & \text { (proportion of } \\
K) & \end{array}$ & 1 \\
\hline$R_{\max }$ & Maximum harvest & $\mathrm{kg}$ & 0.5 \\
\hline$R_{\text {unc }}$ & Uncertainty of resource estimations & $\mathrm{kg}$ & 0.1 \\
\hline$v_{b}$ & Birth coefficient & $\mathrm{J}^{-1}$ & 10 \\
\hline$v_{d}$ & Mortality coefficient & $\mathrm{J}^{-1}$ & 10 \\
\hline$z$ & Variation in offspring traits & {$[0, \rightarrow\rangle$} & 0.2 \\
\hline
\end{tabular}




\section{A.7 Submodels}

\section{Grow resource}

Resource grows on sites according to a logistic growth equation,

$$
\frac{d R_{i, j}}{d t}=R_{i, j} r\left(1-\frac{R_{i, j}}{K}\right) .
$$

Each time-step the sites are updated using the analytical solution of this equation,

$$
R_{i, j}=\frac{R_{i, j}^{*} K e^{r \Delta t}}{K+R_{i, j}^{*} e^{r \Delta t}}
$$

Note that several processes can change the resource on a site during the same timestep. In order to keep the notation simple, we use $R_{i, j}^{*}$ to denote the resource before the update in each of these processes, instead of having different symbols for the resource before and after each of the separate processes.

\section{Diffuse resource}

The resource diffuses following Fick's second law (Fick 1855),

$$
\frac{d R_{i, j}}{d t}=D \nabla^{2} R_{i, j}
$$

with $\nabla=\left(\frac{\partial}{\partial x}, \frac{\partial}{\partial y}\right)$. The equation is discretised in space using the central difference and discretised in time using a forward Euler algorithm. This yields,

$$
R_{i, j}=\left(1-\frac{4 \Delta t}{\Delta x^{2}} D\right) R_{i, j}^{*}+\frac{\Delta t}{\left(\Delta x^{2}\right)} D \sum_{<n n>} R_{i^{\prime}, j^{\prime}}^{*}
$$

where the sum runs over the 4 nearest neighbours. It is shown by von Neumann stability analysis that the solution is stable for $\frac{\Delta t}{\Delta x^{2}} D<\frac{1}{4}$.

\section{Observe}

Agents estimate the resource levels on their present location and the 4 Von Neumann nearest neighbours. The difference between the estimated and the actual amount is drawn from a normal distribution with mean 0 and standard deviation equal to the parameter $R_{\text {unc }}$.

$$
R_{i, j}^{a}=R_{i, j}+\Delta R
$$

with $R_{i, j}^{a}$ the estimated amount, $R_{i, j}$ the actual amount and the difference $\Delta R \sim$ $N\left(0, R_{u n c}\right)$. The result of the observation is stored by the agent until it is replaced by the next observation. 


\section{Harvest?}

The decision of whether to harvest is based on the current internal energy of the agent and the amount of resource that the agent estimates to be present on the site. The current internal energy is compared with the energy maintenance per time-step,

$$
H\left(E^{a}\right)=\frac{E^{a}}{E_{m}}-1
$$

A small value of $H$ indicates that the agent has a low internal energy and needs to harvest soon (i.e. the agent is hungry). If $H$ is negative, then the agent must harvest to survive for another time-step. An agent compares perceived resource on its present site to the maximum possible harvest $R_{\max }$, which is constant,

$$
G\left(R_{i, j}^{* a}, n_{i, j}\right)=1-\frac{R_{i, j}^{* a}}{n_{i, j} R_{\max }} .
$$

The resource that the agent expects to harvest is given by the estimated amount of resource on the patch divided by the total number of agents on the patch. If $G$ is close to 1 , then the expected harvest is small. If $G$ is close to zero, then the agent expects to harvest close to the maximum possible amount. The final probability to harvest is computed based on the functions $H$ and $G$,

$$
P_{\text {harvest }}^{a}=e^{-w_{h}^{a} H G}
$$

where $w_{h}^{a}$ represents the internal tendency of the individual agent to harvest. Thus an agent is likely to harvest if it is hungry, if it sees a good opportunity for making a harvest, and if it has a high internal tendency. Since the functions $h$ and $g$ are dimensionless, the state variable $w_{h}^{a}$ is also dimensionless. Note that the probability of harvesting goes to one if the agent is very hungry, or if the expected harvest is equal to the maximum possible harvest.

\section{Move?}

When deciding whether or not to move, an agent predicts how much resource it expects to obtain at its current site and at the four nearest neighbouring sites. For its current site $i, j$ the agent expects that the resource will be shared evenly among the agents on that site,

$$
E_{e x p, i, j}^{a}=\frac{c R_{i, j}}{n_{i, j}} .
$$

For a neighbouring site, the agent expects that it will share the resource with the agents already present at the site. The agent also considers that it will first have to spend energy to move to a neighbouring site before harvesting there. Thus, the net expected energy gain for the neighbouring site is equal to the expected harvest minus the cost of moving to the site,

$$
E_{e x p, k, l}^{a}=\frac{c R_{k, l}}{n_{k, l}}-E_{m o v e} .
$$


The expected energy gain of the current site is then compared with that of the nearest neighbour with the highest expected gain. Based on this comparison, the probability of moving is computed

$$
P_{\text {move }}=e^{-w_{m}^{a} E_{i, j} / E_{k, l}} .
$$

Thus, an agent is likely to move if the expected energy gain is higher on a neighbouring site than on the present location. Since both the expected harvests have the dimensions of energy, the variable $w_{m}^{a}$ is dimensionless. This variable represents the differences between agents in their likeliness to move.

\section{Harvest}

The harvest is carried out by updating the amount of resource on the harvested site $i, j$ and the internal energy level of the harvesting agent. The agent harvests any resource present on the site up to a maximum of $R_{\text {max }}$. The new amount of resource is thus $R_{i, j}=R_{i, j}^{*}-R_{h}$, with $R_{i, j}^{*}$ the amount before harvesting and $\mathrm{Rh}$ the harvested amount,

$$
R_{h}= \begin{cases}R_{i, j}^{*} & \text { if } R_{i, j}^{*}<R_{\max } \\ R_{\text {max }} & \text { if } R_{i, j}^{*}>R_{\max }\end{cases}
$$

- The cost of harvesting $E_{h}$ is deducted from the internal energy of the agent and the harvested resource is converted with efficiency $c$ and added to the internal energy,

$$
E^{a}=E^{* a}+c R_{h}-E_{h} .
$$

\section{Move}

The agent moves to the nearest neighbour with the highest value of $\frac{R_{i, j}^{a}}{n_{i, j}}$. The location of the agent is updated to the new site.

\section{Maintenance}

For each agent, the daily energy cost of maintenance is deducted from the internal energy,

$$
E^{a}=E^{* a}-E_{m}
$$

If the internal energy of the agent is lower than the maintenance costs, it will become negative after paying maintenance. Because the 'pay maintenance' submodel is immediately followed by the 'die?' submodel, the agent will then die without taking any further actions.

\section{Die?}

Every time-step, agents have a probability of dying or breeding, based on their internal energy. The probability of dying is

$$
P_{\text {die }}\left(E^{a}\right)=e^{-v_{d} E^{a}} .
$$

Thus, the probability of dying goes to one as $E^{a}$ goes to zero. 


\section{Die}

If an agent dies it is removed from the simulation.

\section{Breed?}

If an agent does not die, it has a probability of breeding,

$$
P_{\text {breed }}\left(E^{a}\right)=1-e^{-v_{b}\left(E^{a}-E_{b}\right)}
$$

If $E^{a}<E_{b}$ then the probability is set to zero. The submodel determines whether the agent will breed based on this probability.

\section{Breed}

If an agent breeds, it is replaced by two new agents. The energy of the parent is split evenly among the two offspring. The values of $w_{h}^{a}$ and $w_{m}^{a}$ are inherited by the offspring with some random deviation. The deviation is drawn from a uniform distribution between $-z$ and $z$. In some cases, the resulting value of $w_{h}^{a}$ and $w_{m}^{a}$ can be negative. It is then set to zero instead.

\section{B OFAT results}

The results of the OFAT analysis are shown in Fig 4.10.

\section{C Consequences of the choice of sampling design}

In the Sobol' method, the computation of the sensitivity

$$
V_{i}=V_{x_{i}}\left(E_{x_{\sim i}}\left(y \mid x_{i}\right)\right)
$$

consists of the evaluation of two integrals over the parameter space. Firstly, we keep the parameter $x_{i}$ fixed and compute the expectation value over all other parameters,

$$
E_{x_{\sim i}}\left(y \mid x_{i}\right)=\int f\left(x_{1}, x_{2}, \ldots, x_{k}\right) \prod_{j \neq i}^{k} p_{x_{j}}\left(x_{j}\right) d x_{j}
$$

where the function $y=f\left(x_{1}, x_{2}, \ldots, x_{k}\right)$ gives the model output as a function of the parameters and $p_{x_{j}}(x j)$ is the probability distribution of the parameter $x_{j}$. Secondly, we compute the variance of Eq 4.26 over all values of

$$
V_{x_{i}}\left(E_{x_{\sim i}}\left(y \mid x_{i}\right)\right)=\int\left(E_{x_{\sim i}}^{2}\left(y \mid x_{i}\right)\right) p_{x_{i}}\left(x_{i}\right) d x_{i}-E(y)^{2}
$$

where $\sim i$ indicates 'not $i$ '. Here we have used the law of total expectation to write

$$
\int\left(E_{x_{\sim i}}\left(y \mid x_{i}\right)\right) p_{x_{i}}\left(x_{i}\right) d x_{i}=E(y)^{2}
$$



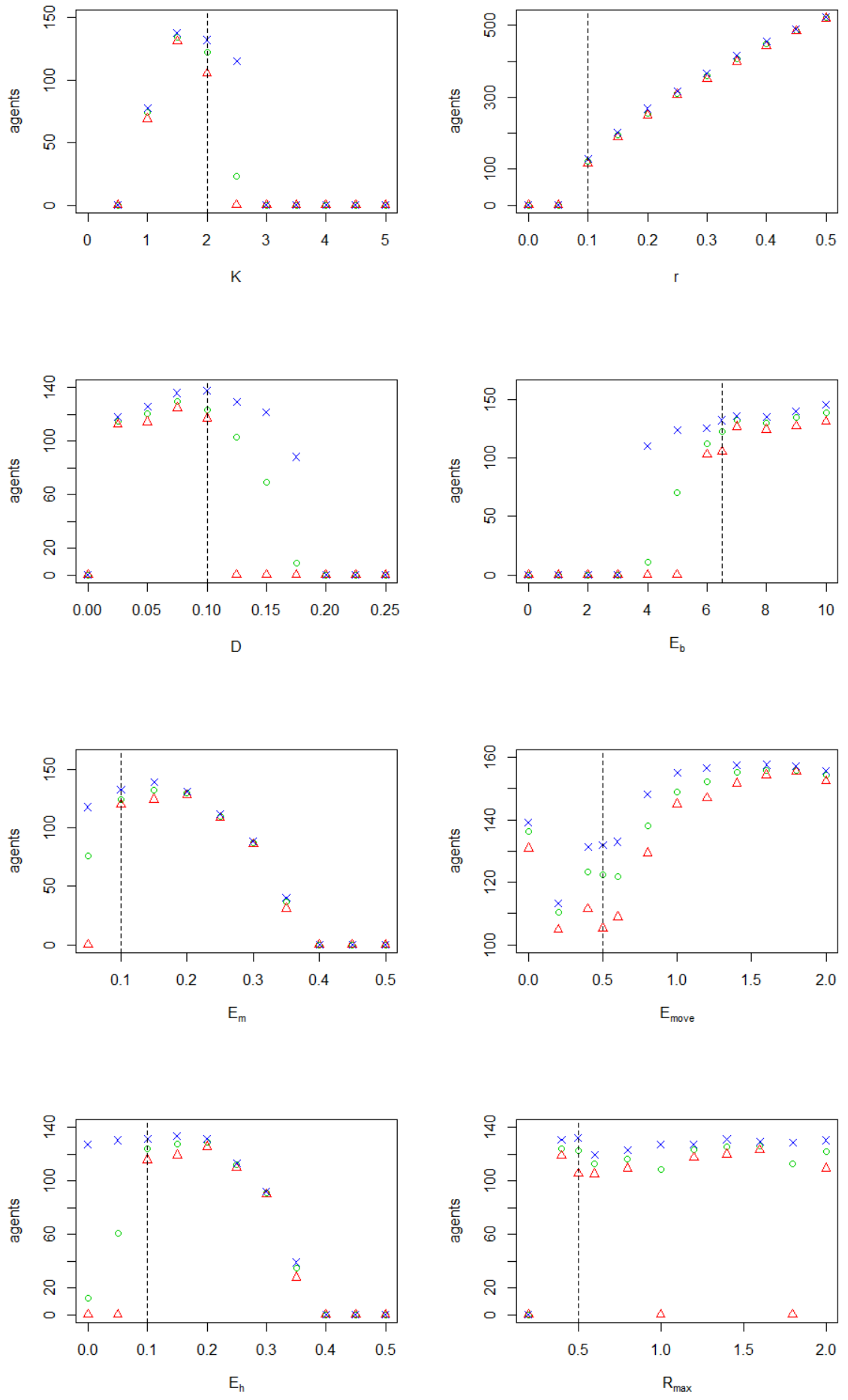

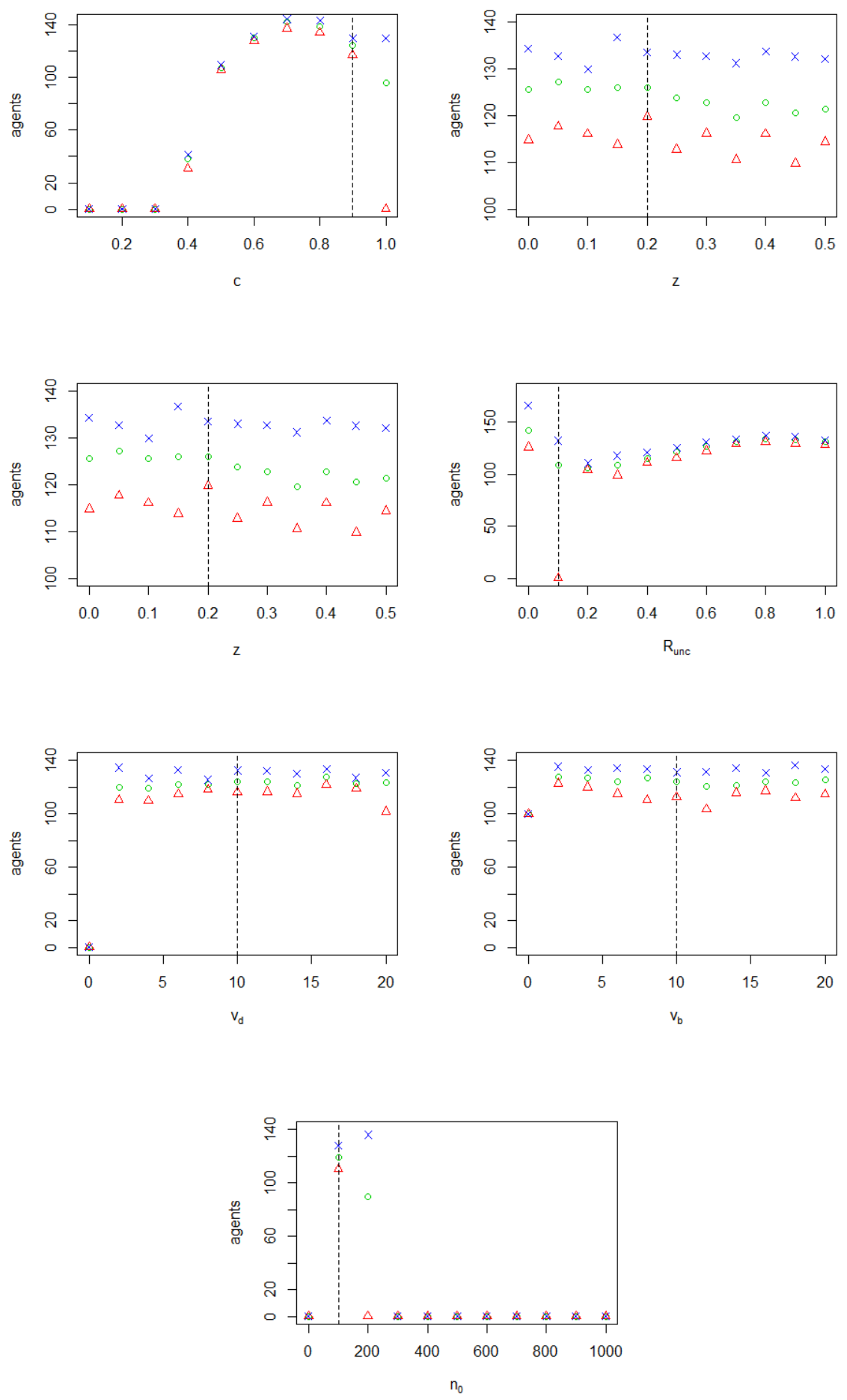

Figure 4.10: Results of the OFAT analysis for all model parameters. Displayed are the mean (green circle), minimum (red triangle) and maximum (blue cross) of the number of agents among 10 replicates. The dashed line represents the nominal value. 
For ABMs we usually have no analytic expression for $f\left(x_{1}, x_{2}, \ldots, x_{k}\right)$. Instead we obtain the function value for a given set of parameter values by running the ABM. Eq 4.25 therefore cannot be evaluated analytically, but is estimated using sampling methods. The most straightforward method to compute these integrals uses a fullfactorial design (Fig 4.11). Every parameter then takes a number of set values and all the possible combinations of parameter values are sampled. The expectation value (Eq 4.26) is then computed for each value of $x_{i}$ by summing over all values for the other parameters. The variance (Eq 4.27) is computed over the possible values of $x_{i}$. The required number of models runs is equal to $N=n_{i}^{m}$, where $n_{i}$ is the number of values for each parameter and $m$ the number of parameters. This is feasible only for models with a small number of parameters. For the test case discussed in this paper, we have $m=15$. For $n_{i}=5$, this would require $N \approx 3.05 * 10^{1} 0$ model runs, which is outside the range that we can realistically perform. This problem is not straightforwardly solved by using a Latin Hypercube sample (Fig 4.11), because we then have only a single sample point for any value of $x_{i}$ and cannot compute the expectation value over different values of $x_{i}$. A number of designs have therefore been suggested to estimate Eq 4.25 based on fewer model runs (e.g. Cukier et al. (1978), Tarantola et al. (2006), Saltelli (2002)). We use the design by Saltelli (2002). For this design, we rewrite the first term on the right side of Eq 4.27 (see Saltelli (2002) for proof).

$$
\begin{aligned}
& \int\left(E_{x_{\sim i}}^{2}\left(y \mid x_{i}\right)\right) p_{x_{i}}\left(x_{i}\right) d x_{i}= \\
& \int f\left(x_{1}, x_{2}, \ldots, x_{k}\right) f\left(x_{1}^{\prime}, x_{2}^{\prime}, \ldots, x_{i-1}^{\prime}, x_{i}, x_{i+1}^{\prime}, \ldots x_{k}^{\prime}\right) \prod_{j=1}^{k} p_{x_{j}}\left(x_{j}\right) \prod_{j \neq i}^{k} p_{x_{j}}\left(x_{j}^{\prime}\right) d x_{j}^{\prime}
\end{aligned}
$$

Observe that all $x_{j}$ have an alternative value $x_{j}^{\prime}$ except the one for which we compute the sensitivity (indicated by $x_{i}$ ). The integral computes the expectation value of $f\left(x_{1}, x_{2}, \ldots, x_{k}\right) f\left(x_{1}^{\prime}, x_{2}^{\prime}, \ldots, x_{i-1}^{\prime}, x_{i}, x_{i+1}^{\prime} . ., x_{k}^{\prime}\right)$ and is evaluated using a single Monte Carlo sample. We randomly draw a number of sample points from the parameter distributions (Fig 4.11) and run the model for each point to obtain a number of model outcomes

$y^{j}=f\left(x_{1}^{j}, x_{2}^{j}, \ldots, x_{k}^{j}\right), \quad y^{\prime j}=f\left(x_{1}^{\prime j}, x_{2}^{\prime j}, \ldots, x_{i-1}^{\prime j}, x_{i}^{j}, x_{i+1}^{\prime j}, \ldots x_{k}^{\prime j}\right) \quad$ for $\quad j=1,2, \ldots, N_{s}$

with $N_{s}$ the chosen sample size. The integral is then estimated by averaging the product $y_{j} y_{j}^{\prime}$ over all sample points. Eq 4.30 implies that the partial variance of Eq 4.25 is equal to the covariance between $f\left(x_{1}, x_{2}, \ldots, x_{k}\right)$ and $f\left(x_{1}^{\prime}, x_{2}^{\prime}, \ldots, x_{i-1}^{\prime}, x_{i}, x i+1^{\prime} . ., x_{k}^{\prime}\right)$, where the primed parameter values are sampled independently from the values without a prime. However, the estimates for the covariance can yield negative values because of this independent sampling. In contrast, the partial variance can never be negative because it consists of squared deviations. Thus, the appearance of negative values for the estimates of the sensitivity indices results from the inaccuracy of the covariance estimate. This inaccuracy is often substantial, as we illustrate below for a simple example. This may be because the integral of $\mathrm{Eq} 4.30$ is computed over a parameter space of dimension $2 k-1$. The introduction of the primed parameter 
values increases the dimension of the parameter space from which we sample, and therefore makes the coverage of the parameter space thinner.

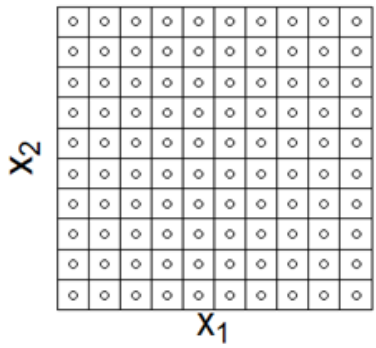

(a)

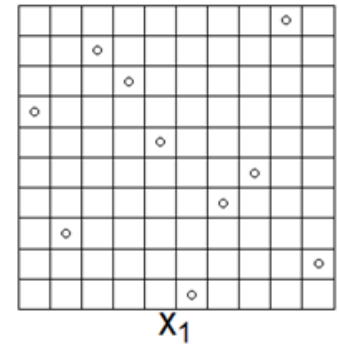

(b)

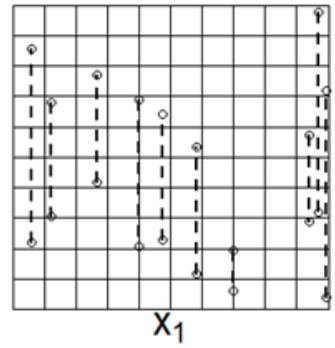

(c)

Figure 4.11: Different designs to sample from the parameter space. The full-factorial design (a) samples all combinations of a number of set values for each parameter (here we show only two parameters) and therefore allows for the computation of the sensitivity index for any of those parameters. The LHS design (b) samples only one value for each parameter. It does not allow for straightforward computation of the Sobol' indices, because Eq 4.25 asks for the computation of the expectation value of the model outcome while keeping one parameter fixed. In the full factorial design this would correspond to evaluation the sum of outcomes over a row or column, but in the LHS design we have only a single value per row or column and therefore cannot evaluate this sum. The design by Saltelli (2002) (c) samples pairs of points keeping one parameter fixed between each pair. The sensitivity index is estimated by the covariance between two vectors, each of which contains the model outcome that corresponds to one sample point out of every pair. The samples in the figure would allow for the computation of the sensitivity to $x_{1}$. More points would have to be sampled to compute the sensitivity to $x_{2}$.

A simple test case illustrates the above discussion. We apply the sampling scheme to the model

$$
y=x_{2}+x_{3}
$$

With $x_{1}, x_{2}, x_{3} \sim U(0,1)$. The parameter $x_{1}$ does not affect the outcome and thus $S_{x 1}=0$. The parameters $x_{2}, x_{3}$ both affect the outcome equally and there are no interaction effects. Thus, $S_{x_{2}}=S_{x_{3}}=0.5$. These values for the sensitivities are verified by analytic evaluation of Eq 4.27. We apply the sampling design by Saltelli (2002) (Fig 4.11) for increasing sample size and compare the outcomes to the analytical values. Besides the sensitivities we also compare the outcomes for the total mean and variance. The relative deviations from the exact mean (i.e. the deviations from the exact mean as a percentage of the exact mean) are below $10 \%$ even for base sample sizes below 100 and below $2 \%$ for larger sample sizes (Fig 4.12a). For the variance, the relative deviations from the exact value are below $10 \%$ for a sample size of the 
order and are within a few percent for larger sample sizes (Fig 4.12b). The sensitivity estimates, however, are less accurate (Fig 4.12c). We encounter deviations from the exact values between 10-20\% even for base sample sizes close to 5000 . We cannot plot the relative deviation for $S_{x_{1}}$ because the analytic value is zero. The absolute estimates for the sensitivity reveal that the distribution is centred around the true value zero; The number of negative outcomes is approximately equal to the number of positive outcomes.

The example model of Eq 4.32 shows that even for a very simple test model, a large sample size is needed to accurately estimate sensitivities following the design by Saltelli (Saltelli 2002). This matches the finding that the sensitivity estimates for our ABM have wide confidence intervals (Fig 4.7). Furthermore, negative sensitivity estimates are not unlikely, especially for parameters that have little effect on the model outcomes. These negative estimates indicate the inaccuracy of the sampling design for estimating the sensitivities. We therefore suggest to report negative sensitivity estimates explicitly. For example, some of the sensitivity indices that we report in Fig 4.7 for our ABM test case have confidence intervals that are largely negative. Discarding these negative ranges by setting them to zero might give the reader an unrealistic confidence in the accuracy of the estimates. 


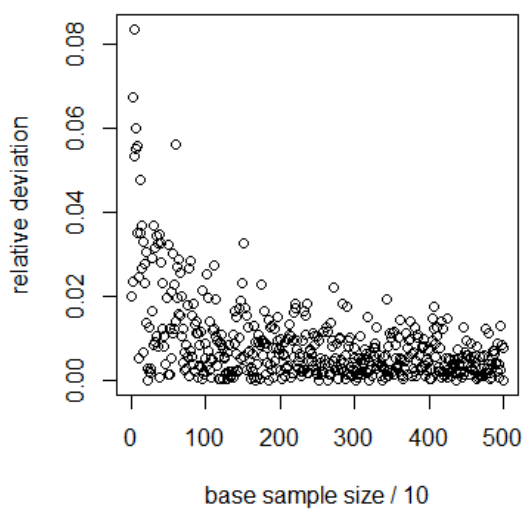

(a)

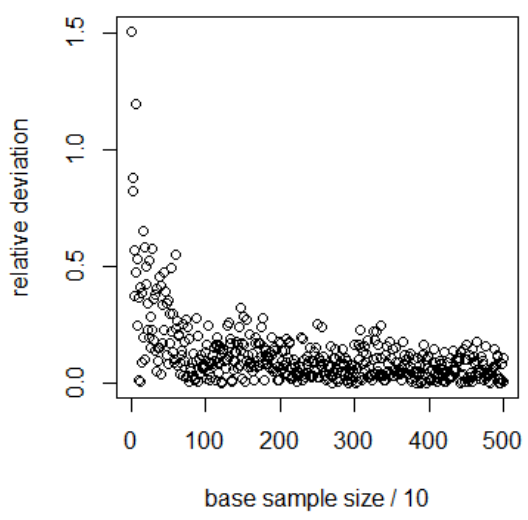

(c)

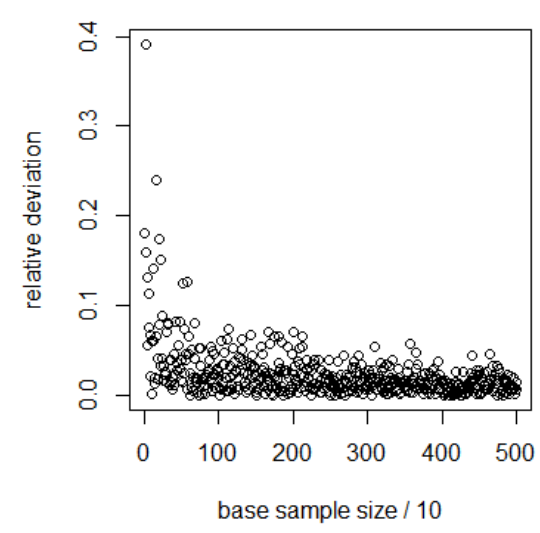

(b)

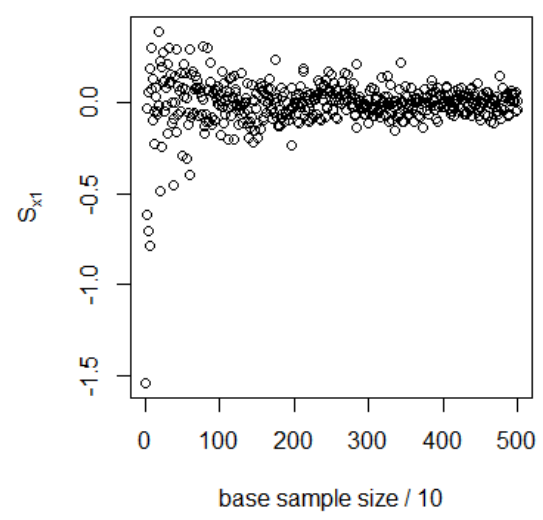

(d)

Figure 4.12: (a):Relative absolute deviation of the estimate of the mean of the model outcomes from the true mean for increasing sample size. For large sample sizes, the estimate lies within $2 \%$ of the true value. (b): Relative deviation of the estimate of the variance of the model outcomes from the true variance for increasing sample size. For large sample sizes, the estimate lies with a few percent of the true value. (c): Relative deviation of the estimate of the sensitivity of $x_{2}$ from the analytic value. Even for large sample sizes the deviations are significant. (d): Estimate of the sensitivity to $x_{1}$ for increasing sample size. The estimates are centred around the (correct) analytic value 0 , but deviate significantly from this value. 


\section{Chapter 5}

\section{Resilience through adaptation}

G.A. ten Broeke, G.A.K. van Voorn, A. Ligtenberg, J. Molenaar.

PlosOne, 12(2):e0171833. 
Adaptation of agents through learning or evolution is an important component of the resilience of Complex Adaptive Systems (CAS). Without adaptation, the flexibility of such systems to cope with outside pressures would be much lower. To study the capabilities of CAS to adapt, social simulations with agent-based models (ABMs) provide a helpful tool. However, the value of ABMs for studying adaptation depends on the availability of methodologies for sensitivity analysis that can quantify resilience and adaptation in ABMs. In this paper we propose a sensitivity analysis methodology that is based on comparing time-dependent probability density functions of output of ABMs with and without agent adaptation. The differences between the probability density functions are quantified by the so-called earth-mover's distance. We use this sensitivity analysis methodology to quantify the probability of occurrence of critical transitions and other long-term effects of agent adaptation. To test the potential of this new approach, it is used to analyse the resilience of an ABM of adaptive agents competing for a common-pool resource. Adaptation is shown to contribute positively to the resilience of this ABM. If adaptation proceeds sufficiently fast, it may delay or avert the collapse of this system.

\subsection{Introduction}

Many social-ecological systems, which provide important ecosystem services, are under increasing pressure from human activities and environmental changes Folke (2006), Rammel et al. (2007). To predict how these systems will respond to pressures, we need to describe their Complex Adaptive System (CAS) characteristics. CAS are systems with many autonomous agents that interact with each other and with their environment Holland (2006). The system-level behaviour of CAS 'emerges' from lower-level interactions and cannot a priori be predicted from the properties of its agents. To properly manage CAS that are under pressure, it is important to understand which properties affect resilience, i.e., the capacity of the system to cope with pressures while maintaining its identity and avoiding drastic changes Walker et al. (2004). It has been shown that some CAS show an initial resilience against pressure, until a tipping point is reached where the system undergoes a drastic transition to an entirely different system state Scheffer (2009). In order to predict the occurrence of such transitions, we need to understand the origin and extent of the resilience of CAS Levin (1998).

Real-world CAS constantly experience the influence of small disturbances, changing conditions, and random events. This means that CAS are never in a static equilibrium situation and their state is continuously changing Walker et al. (2004). Most of these changes are small, and do not affect the organisation of the system fundamentally. Such small changes are commonly described as movements within a 'domain of attraction'. Within a domain of attraction, the system maintains the same qualitative structure and organisation. Often these domains of attraction are illustrated metaphorically as valleys in a potential landscape that describes the state of the system. For example, Fig 5.1a shows a hypothetical potential landscape with two domains of attraction. The ball represents the current state of the system, and 


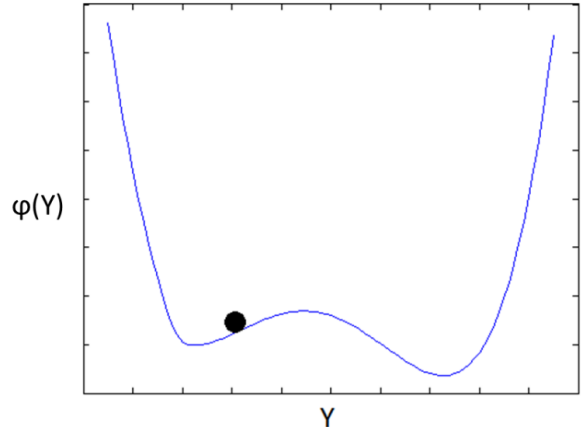

(a)

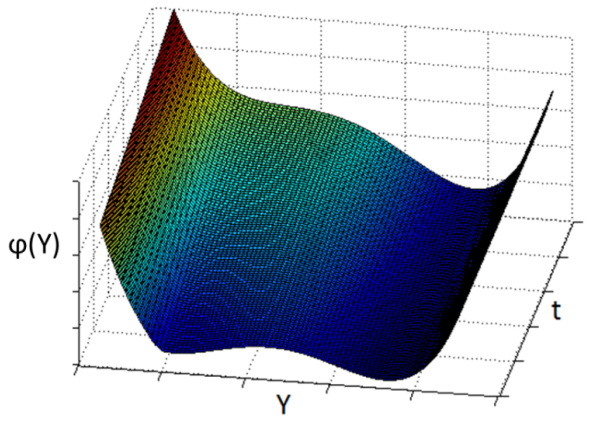

(b)

Figure 5.1: Hypothetical potential landscape $\phi(Y)$ as function of a state variable $Y$. The black ball represents the current system state, and the two valleys correspond to two separate stability domains. (a): In classical resilience studies, such as many engineering applications, the landscape is static. Resilience may be measured in terms of the width and height of the stability domain. (b): In CAS the stability landscape may change over time. In this example, the landscape is identical to (a) at $t=0$. Over time, the leftmost stability domain becomes less resilient and eventually disappears.

is naturally attracted towards the bottom of the domain of attraction. Pressures and shocks of limited strength and duration continuously shake the system within the boundaries of the current domain of attraction, but rarely push the system past those boundaries. Pressures and shocks that are strong and/or frequent, in contrast, are more likely to push the system outside its domain of attraction. Such a tipping point leads to a qualitatively different kind of system state, and may have drastic consequences for the development of the system. The resilience of the system against the occurrence of tipping points is, of course, determined by the shape of the current stability domain. If this stability domain is wide and deep, external pressures are unlikely to cause a tipping point, and the system is said to be resilient. Since tipping points in ABMs are difficult to detect, and the stability domains may change due to adaptation, assessing the resilience of ABMs is a difficult challenge.

\subsection{Materials and Methods}

\subsubsection{Stochastic output}

Since most ABMs are stochastic, each single simulation run of an ABM yields its own output. We use a large number of replicate runs to estimate the range of possible output values. Most sensitivity analysis methods use these replicate runs only to estimate the mean and variance of the model output under various parameter settings (e.g., Cariboni et al. (2007)). If the model output is normally distributed, then the mean and variance indeed fully describe its variation. For ABMs, however, both the underlying model behaviour and the corresponding shape of the output distribution are usually not known a priori. This shape contains information that is important for 


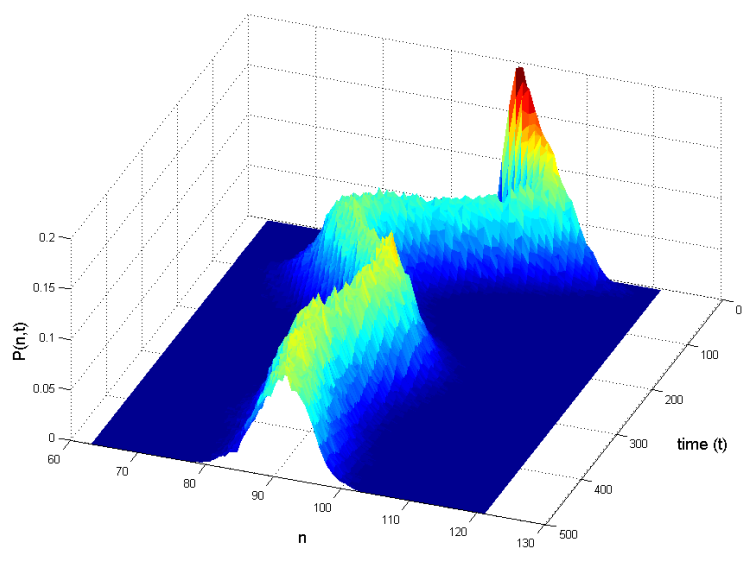

Figure 5.2: Time-dependent histogram that estimates the probability $P(n, t)$ of obtaining some model output $n$ at time $t$. The figure was generated using 10.000 replicate runs. In this example, the output seems to stabilise as a normal distribution around of mean value of $n=90$.

understanding the model behaviour. For example, if a number of model runs undergo a transition into another domain of attraction, this will lead to a bimodal output distribution.

In this paper, we use replicate runs to estimate the output distribution. These estimates will be visualised as histograms. Since the output distributions are timedependent, they are estimated as functions of time. Plots of the time-dependent estimated PDFs visualise the change of the model output over time. For example, the PDF Fig 5.2 initially shows fluctuations over time, after which it converges to a domain of attraction. After this convergence it seems to stabilise as a normal distribution. Since adaptation is a process that takes place over time, its effects are contained in the time-dependent PDF. Critical transitions where the model is pushed into an alternative domain of attraction appear as sudden changes in the PDF.

\subsubsection{Statistical tests}

To assess the characteristics of the estimated output PDFs, we use statistical tests for stationarity and ergodicity. Details of these tests are given in 5.A. Stationarity tests use a number of replicate runs to verify whether an ABM becomes 'stationary', i.e. reaches a state in which its output PDF shows no long-term changes over time Grazzini (2012). For example, in Fig 5.2 the model appears to converge to a stationary state, after some initial fluctuations. If the ABM becomes stationary, the corresponding PDF represents the long-term behaviour of the ABM and there is no adaptation over the considered time-span.

Ergodicity tests compare an output sample of a single model run, which is obtained by recording the model output every time-step, to output samples of a number of replicate runs measured at a set time van Lith (2001), Grazzini (2012). The model is 
Table 5.1: Description of used symbols.

\begin{tabular}{|l|l|l|}
\hline Symbol & Description & Units \\
\hline$D$ & Diffusion coefficient & $\mathrm{km}^{2}$ day $^{-1}$ \\
$d_{e}$ & Earth mover's distance & - \\
$d_{J}$ & Jensen-Shannon distance between histograms & - \\
$d_{u}$ & Euclidian distance between histograms & - \\
$d_{(j, k)}$ & Distance between bins of histograms (5.B) & - \\
$E_{h}$ & Energy cost for an agent to harvest & $\mathrm{J}$ \\
$E_{m}$ & Energy cost for an agent to move & $\mathrm{J}$ \\
$g_{(j, k)}$ & Flow between bins of histograms (5.B) & - \\
$H_{t}$ & Vector used in runs test (5.A) & - \\
$j, k, l$ & Indices for output values & - \\
$N$ & Number (observations, model runs,... $)$ & $\#$ \\
$n$ & Number of agents & $\#$ \\
$P_{a}(n, t)$ & Normalised histogram of ABM with adaptation & - \\
$P_{b}(n, t)$ & Normalised histogram of ABM without adaptation & - \\
$Q_{i}(t)$ & Adaptation measure for parameter i at time t & - \\
$Q_{i}^{\prime}(t)$ & Rate of adaptation over time & $\mathrm{t}$ \\
$T$ & Length of time-series & - \\
$t$ & Time index & day \\
$V$ & Variance & - \\
$W$ & Statistic used in trend test (5.A) & - \\
$w_{h a r v e s t}$ & Agent's harvest parameter & - \\
$w_{m o v e}$ & Agent's move parameter & - \\
$Y$ & Model output variable & - \\
$z$ & Inheritance parameter & - \\
$\lambda$ & Extinction parameter & $\mathrm{d}$ \\
$\mu$ & Mean & - \\
$\rho$ & Increase in pressure & Potential \\
$\phi$ & & -1 \\
\hline
\end{tabular}


considered to be ergodic if the null-hypothesis that the output samples are identical is not rejected. In other words, the stochastic variation between replicates is then equal to the stochastic variation over time of a single model run. A difference between the output samples indicates that the model output contains variation over time that cannot be attributed to stochasticity, and may indicate that adaptation has taken place over the considered time-scale. Since only a single model run is used to estimate the time-averaged PDF, ergodicity is a highly attractive property if we want to explore the model behaviour on very long time-scales. In the following, we will refer to histograms that are obtained by recording a single model run over successive times-steps as time-averaged histograms. We refer to histograms that are recorded over replicate runs as function of time as time-dependent histograms.

\section{Sensitivity measures}

In order to assess how adaptation influences the model output, we need to measure the sensitivity of the output PDF to the presence of adaptation. We measure this sensitivity by comparing the estimated time-dependent PDFs of the output of model versions with adaptation $P_{a}(n, t)$ and without adaptation $P_{b}(n, t)$. Here $n$ denotes the number of agents, which is used throughout this paper as the central model output. To quantify the comparison we use the earth-mover's distance $d_{e}$ Rubner et al. (1998).

For an intuitive interpretation of the earth mover's distance, consider two PDFs $P_{a}(n, t)$ and $P_{b}(n, t)$ as amounts of mass that are spread over a distance specified by the model output $n$. The earth-mover's distance $d_{e}\left(P_{a}(n, t), P_{b}(n, t)\right)$ is then the work required to transform $P_{a}(n, t)$ into $P_{b}(n, t)$. Several properties follow from this interpretation. Firstly, for all $P_{a}(n, t)$ and $P_{b}(n, t), d_{e}\left(P_{a}, P_{b}\right) \geq 0$, and $d_{e}\left(P_{a}, P_{b}\right)=0$ implies that $P_{a}=P_{b}$. Any positive value of $d_{e}\left(P_{a}, P_{b}\right)$ thus indicates a difference between the PDFs. Small values indicate that the PDFs are quite similar, and large values indicate strong differences between the PDFs. We denote by

$$
Q(t)=d_{e}\left(P_{a}(n, t), P_{b}(n, t)\right)
$$

the effects of adaptation at time $t$. Note that all the used symbols are described in Table 5.1. $Q(t)$ measures the effect of a parameter change, namely the enabling or disabling of adaptation. Other sensitivity measures based on a comparison between output PDFs have been previously suggested (e.g. Liu et al. (2006), Auder and Iooss (2008), Borgonovo (2007)). All of these measures are based on the (lack of) overlap between those PDFs. For our purposes, these measures are not suitable, because we want to measure the effects of adaptation even when there is little or no overlap between the PDFs. The earth-mover's distance allows us to do this, because an increase in the distance between PDFs will result in an increase of the earth-mover's distance, even when the PDFs do not overlap.

In addition to the effects of adaptation at a specific time, we are also interested in the rate of adaptation. We calculate the rate of change of $Q(t)$ by computing the difference between time-steps,

$$
Q^{\prime}(t)=d_{e}\left(P_{a}(t), P_{b}(t)\right)-d_{e}\left(P_{a}(t-\Delta t), P_{b}(t-\Delta t)\right)
$$


with $\Delta t$ small compared to the time-scale of the process. This measure for the rate of adaptation helps to identify periods in time where adaptation proceeds relatively fast, or where there is little adaptation. For example, adaptation may be influential on short simulation times, but have little effect on longer simulation times, or the other way around.

\subsubsection{Computation of the earth mover's distance}

Computing the earth-mover's distance between a pair of distributions amounts to finding the minimal 'work' needed to change one distribution into the other. We write $g(j, k)$ for the matrix element that contains the flow between output values $j$ and $k$, $d(j, k)$ for the distance between the output values, and we consider $g(j, k) d(j, k)$ as the work required to transport $g(j, k)$ from $j$ to $k$. There are many possible choices for the matrix $g$ to transform one distribution into the other. To compute the earth-mover's distance, we minimise $g(j, k) d(j, k)$ over these possible choices Ling and Okada (2007),

$$
d_{e}=\min \left(\sum_{(j, k)} g(j, k) d(j, k)\right)
$$

with the constraints

$$
\begin{gathered}
\sum_{k} g(j, k)=P_{a}(j) \quad \forall j \\
\sum_{j} g(j, k)=P_{b}(k) \quad \forall k \\
g(j, k) \geq 0 \quad \forall j, k
\end{gathered}
$$

with $d(j, k)$ the distance between $j$ and $k$, and the indices $j$ and $k$ running over their domains. Eq 5.4 and Eq 5.5 ensure that the flow is such that the distribution $P_{a}(j)$ is transformed into $P_{b}(k)$. Eq 5.6 ensures that mass is moved from $P_{a}(j)$ to $P_{b}(k)$, and not the other way around. Eq 5.3 ensures that the flow is chosen such to minimise the required 'work' $g(j, k) d(j, k)$. For two PDFs of a single output variable, this minimisation is accomplished by going through all consecutive pairs of output values, and keeping track of the amount of mass that needs to be transported. 5.C contains a sample pseudo-code for this computation. For the computation of $d_{e}$ for continuous, or multi-dimensional distributions, we refer to Venkatasubramanian (2013). Packages to compute $d_{e}$ are available for various statistical software and programming languages.

\subsubsection{Method to measure adaptation}

In Fig 5.3 we present the scheme of our method to measure adaptation of ABMs. The first step is to prepare an ABM with adaptation, and a version of the same ABM in which this adaptation is disabled. Agent adaptation in our test-case consists of the selection of agent characteristics for replication or modification Levin (1998). Disabling this selection ensures that there is no adaptation and that the distribution of 
agent properties does not change over time. In the ABM with adaptation, the agent properties may change over time, for example through natural selection, or through individual agents learning from past experience or imitation of other agents Holland (2006), Macal (2016). To measure the effects of such adaptations, we compare the output of the ABM with adaptation to that of the ABM without adaptation. The rest of the method is composed of steps for analysing the model output and comparing the estimated output PDFs using Eq 5.1 and Eq 5.2.

Based on a large number of replicate runs, we generate histograms of the output to estimate the time-dependent output PDFs of the ABM without adaptation $P_{b}$ and of the adaptive ABM $P_{a}$. To measure adaptation, we need to examine how the PDFs change with time. Thus, for each ABM we estimate the PDF at multiple points in time. Based on these data, we use stationarity tests to verify whether the ABMs reach an equilibrium, or continue to change over the considered time-scale. Furthermore, for each ABM we also perform a single model run with a very long simulation time to test the ergodicity. If the stationarity test reveals that the ABM reaches a stationary state, then the time after which this state is reached is used as a starting point for the ergodicity test, but the ergodicity test explores longer simulation times than the stationarity test. If the model is ergodic, then we may conclude that no adaptation takes place.

Once the PDFs of the ABMs with adaptation and without adaptation have been analysed, we proceed by comparing them to quantify the effects of adaptation. The difference between the PDFs is quantified using $Q$ (Eq 5.1). In addition to measuring the effects of adaptation over a specified time-span, we also measure the rate of adaptation using $Q^{\prime}$ (Eq 5.2). This rate of adaptation is especially relevant in the context of resilience. For the resilience of the system to be influenced by adaptation, the rate of adaptation must be sufficiently fast to respond to pressures. For example, if a pressure to the system increases very fast, then adaptation must also be able to occur fast in order to affect the consequences of the pressure.

\subsubsection{Model description}

We consider a previously published test-case, in which adaptation emerges through a process of natural selection. An overview of all model parameters and their default values is given in 5.D. For a full model description we refer to Ten Broeke, Van Voorn and Ligtenberg (2016). Here we focus mainly on the mechanism for adaptation. Fig 5.4 presents a flow chart of the model. The test-case is a resource-agent system, in which the agents compete for a renewable common-pool resource in a spatial environment. The spatial environment is composed of a grid of square sites, on which resource grows and diffuses, and on which agents live (Fig 5.5). Every timestep, each agent estimates the amounts of resource and observes the number of agents on its present location and the four neighbouring sites. Based on this information, it decides whether to harvest on its present site, to move to a neighbouring site, or to stay inactive. These decisions are stochastic, but the probabilities are influenced by the agent's own (decreasing) internal energy state ('hunger') as well as the state of the local surroundings, including the presence of resources ('food availability') and other agents ('crowding'). Harvesting or moving costs energy, in addition to the energy consumed by basic maintenance every time-step. There are thus trade-offs be- 


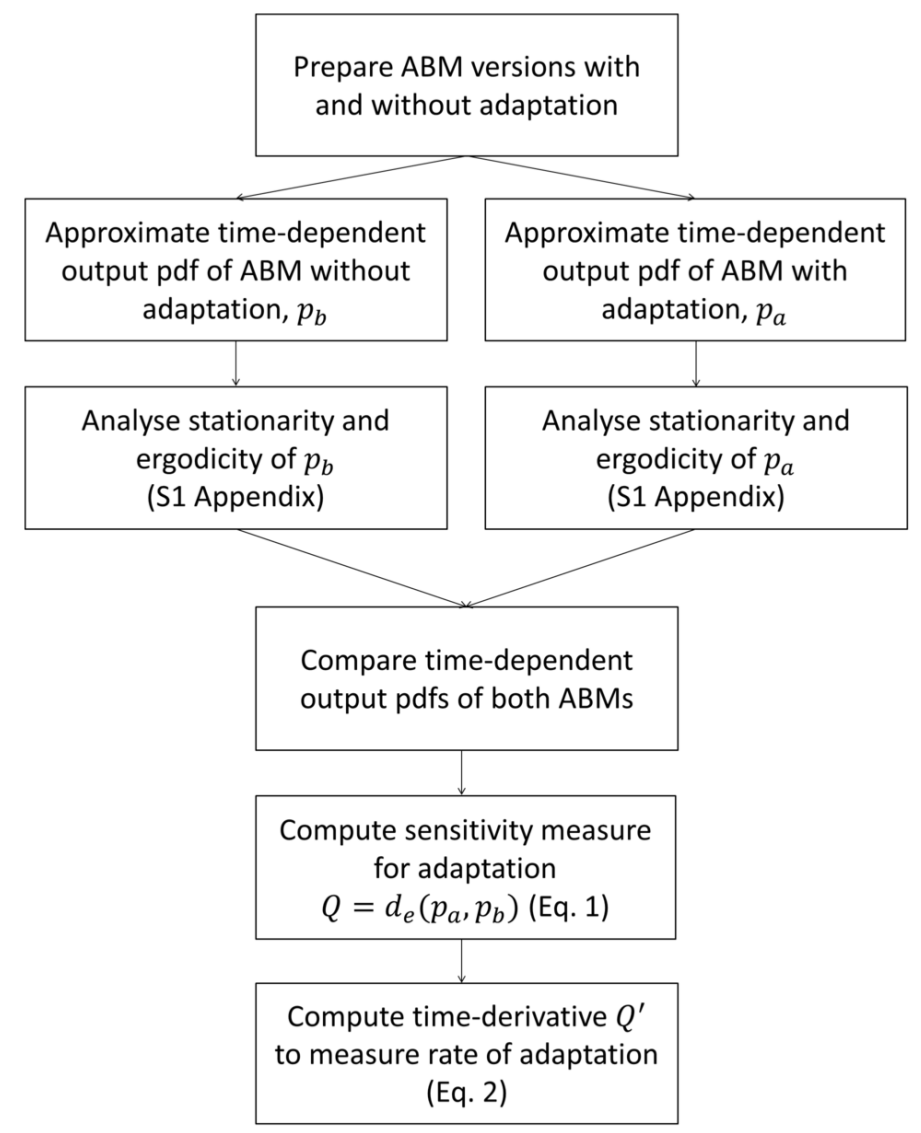

Figure 5.3: Flow chart of the proposed method to measure adaptation. The method is based on a comparison between versions of an ABM with and without adaptation. See the main text for further explanation. 
tween harvesting, moving, and remaining inactive, which form the basis of the agent's decision-making process. At the end of each time-step, all agents have a probability of dying and of reproducing, based on their internal energy state. Low values for the internal energy increase the probability of dying, whereas high values increase the probability of reproducing.

Each agent has two agent parameters that affect its decisions to harvest or move: $w_{\text {harvest }}$ and $w_{\text {move }}$. Besides these parameters, the probabilities of harvesting or moving also depend on the weighted average of a few factors, including the agent's internal energy, the resource on its site and the resource on its neighbouring sites. This average, multiplied by $w_{\text {harvest }}$ or $w_{\text {move }}$, is input to a function that determines the probability. If $w_{\text {harvest }}=0$ the agent will choose to harvest with probability 1. Similarly, if $w_{\text {move }}=0$ the agent will choose to move with probability 1 . Larger parameter values decrease the probabilities. For the initial agent population, the values of these parameters are drawn from a uniform probability distribution. The values remain constant over the lifetime of an agent. Upon reproduction, an agent passes on its values of $w_{\text {harvest }}$ and $w_{\text {move }}$ to its offspring, with a small random deviation. The distribution of the parameter values across the population may thus change over time through a process of natural selection. Since the probability of reproducing is higher for agents with a high internal energy, agents that are successful at gathering energy are also more likely to reproduce and pass on their characteristics. Agents that are not successful at gathering energy, in contrast, are more likely to die without reproducing. Parameter values that increase an agent's success at gathering energy are thus passed on more frequently, whereas parameter values that decrease its success tend to disappear from the population. Over time, the distribution across the population of the parameters values will thus move towards values that increase success at gathering energy. This change in the distribution represents the process of the population adapting to its environment.

To obtain a non-adaptive version of the ABM, we disable the inheritance of agent characteristics. In the non-adaptive version, when a new agent is added to the system, its values of $w_{\text {harvest }}$ and $w_{\text {move }}$ are chosen according to the same probability distribution that is used for the initial agent population. Thus, in this version there are no long-term changes in the distribution of $w_{\text {harvest }}$ and $w_{\text {move }}$ across the agent population.

\subsection{Results}

\section{Non-adaptive ABM}

In the following sections, we will consider the agent population size $n$ as the central model output. Following the scheme in Fig 5.3, we estimate the output histogram of the ABM without adaptation in the default parameter setting. Detailed results of the statistical tests are given in 5.C. Model runs in the default parameter setting show that the output initially oscillates, but stabilises around $t=1000$. Between $t=1000$ and $t=2000$, the mean of the output across replicates is nearly constant on average, with short-term fluctuations. Due to these fluctuations, stationarity tests show that the output is not stationary. To test whether there are any long-term trends, we average the output over short time-windows and test whether the series of window 


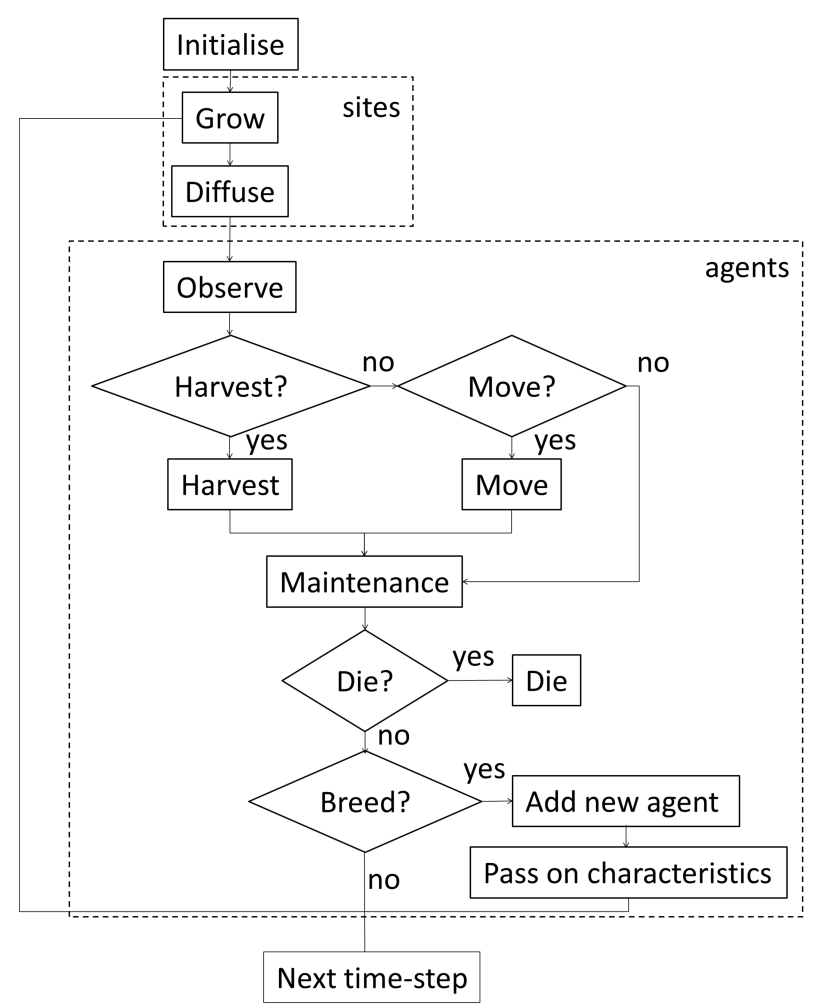

Figure 5.4: Model flow chart. The dashed boxes indicate loops over all sites or all agents. For detailed explanation we refer to Ten Broeke, Van Voorn and Ligtenberg (2016). 


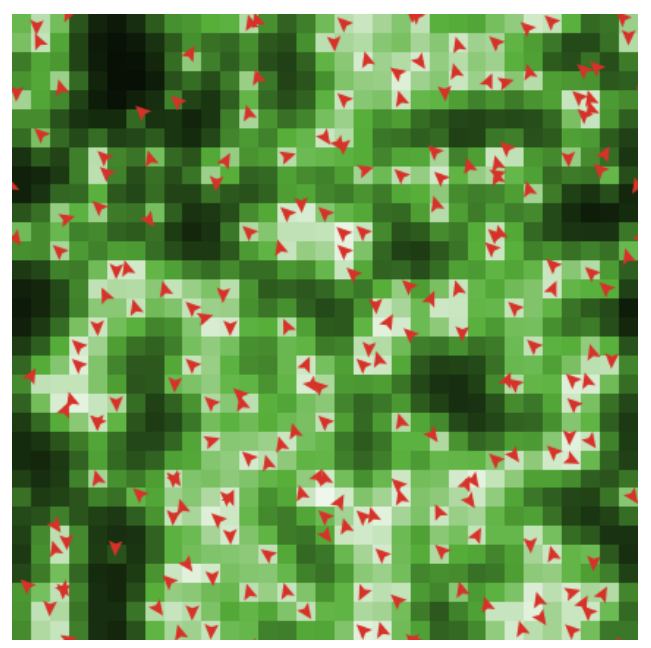

Figure 5.5: Snapshot of a typical simulation run of the ABM test-case. The ABM is composed of a square grid of sites. Dark colours indicate sites with high resource densities, while light colours indicate low densities. The red arrows show the current locations of agents.

means is stationary Bendat and Piersol (2011). These tests indicate that between $t=1000$ and $t=2000$ the window means are stationary.

To test the ergodicity we compare the histogram $P_{b}$ of the output over replicate runs at $t=1000$, to the histogram of a single model run, ranging from $t=1000$ to $t=$ 100,000. The test confirms that the ABM without adaptation is ergodic, indicating that it shows no long-term change even on simulation times up to 100,000 time-steps (Fig 5.6a). The histogram over replicate runs at $t=1000$ and the time-averaged histogram thus both estimate the stationary PDF of the ABM without adaptation, and describe the behaviour of this ABM on long time-scales.

\subsubsection{Adaptive ABM}

The stationarity test for the adaptive ABM shows similar results as the ABM without adaptation. The model output has stabilised around $t=1000$ and appears to be stationary between $t=1000$ and $t=2000$. The output histogram corresponding to the stationary state is approximately equal to the histogram of the ABM without adaptation.

Although the stationarity test does not reveal any effects of adaptation, it is possible that adaptation proceeds so slowly that the time period between $t=1000$ and $t=2000$ is too short to observe its effects. We use an ergodicity test to explore the behaviour of the ABM with adaptation on longer time-scales. The results reveal that the model output is indeed affected by adaptation on simulation times between $t=1000$ and $t=100,000$. The time-averaged histogram of a model run with a long simulation time differs significantly (5.C) from the histogram over replicate runs at $t=1000$ (Fig 5.6b). Over time the agent population gradually adapts, causing a significant increase in the population size. So we observe that, while the system 


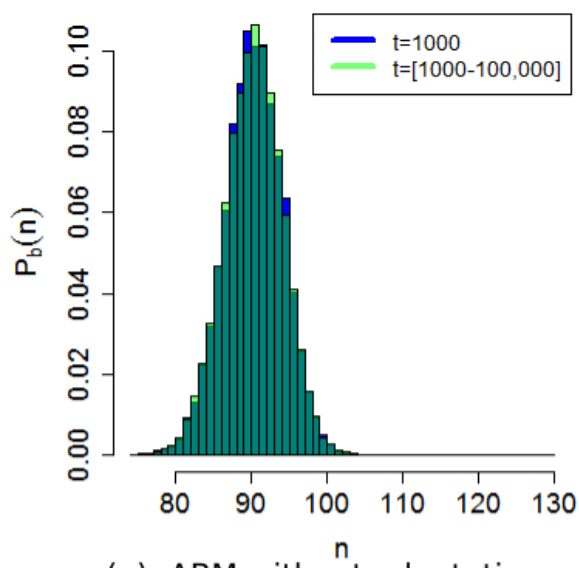

(a): ABM without adaptation

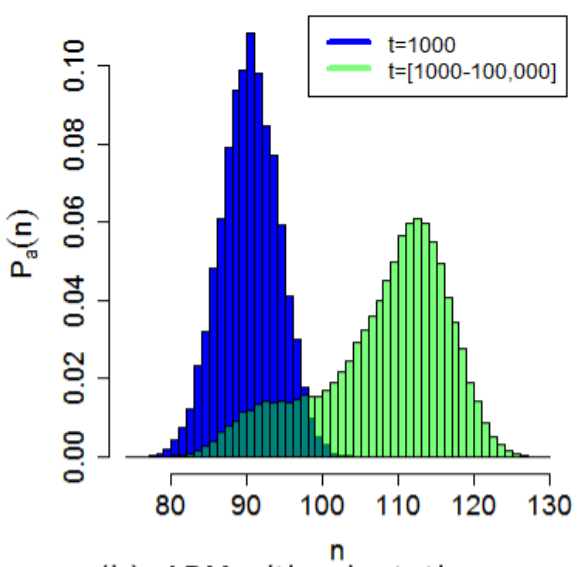

(b): ABM with adaptation

Figure 5.6: Time-averaged histograms of the number of agents $(n)$. The blue histograms are measured over 10,000 replicate runs at $t=1000$, and the green histograms over the time-steps of a single model run between $t=1000$ and $t=100,000$. All parameters are at their default values. Fig 5.6a indicates that the ABM without adaptation is ergodic, and Fig 5.6b indicates that the ABM with adaptation is not ergodic.

remains in the same stability domain on long time-scales, adaptation causes the shape of this stability domain to gradually change over time, whereas without adaptation the shape of the stability domain remains constant.

\subsubsection{Effects of adaptation}

To estimate the effects of adaptation we compute $Q$ between the estimated timedependent PDFs $P_{a}$ and $P_{b}$. We use a total simulation time of 100,000 time-steps, and record the output every $\Delta t=100$ time-steps. Fig 5.7a shows that the distance between the PDFs increases on long time-scales. The corresponding values of $Q$ (Fig 5.7b) show that the distance between the PDFs initially increases, but then decreases approximately between $t=5,000$ and $t=15,000$, after which it increases again. The initial increase of $Q$ is caused by a small decrease in the output of the ABM with adaptation. After this initial decrease in the output, the output starts to increase, first moving towards the ABM without adaptation, and then becoming larger. The initial decrease of the number of agents in the adaptive ABM is caused by increased competition between the agents, which tend to harvest more often. On longer time-scales, the agents adapt to move less often, and wait at the same location to let the resource grow before harvesting. Since agents mostly stay in their location, there is a decreased competition between the agents, which leads to an increase in the number of agents on longer time-scales. This learning process continues until the simulation is stopped at $t=100,000$ as is shown by the values of $Q$ and $Q^{\prime}$.

To demonstrate why we prefer the earth-mover's distance over some other measures, we have reproduced Fig 5.7b using two other commonly used measures for the 


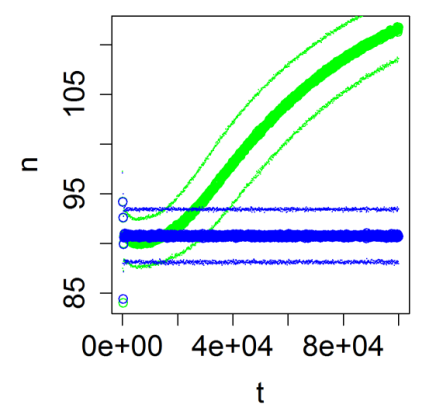

(a)

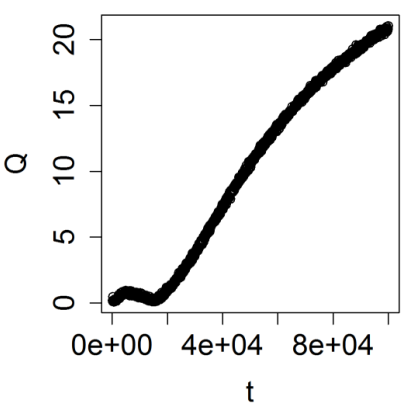

(b)

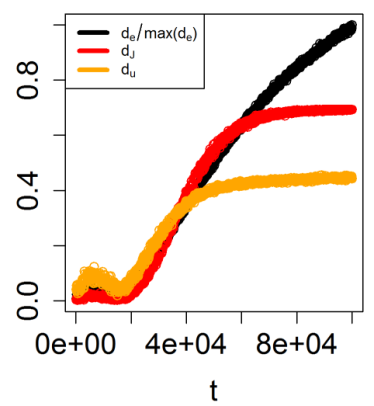

(c)

Figure 5.7: (a): Means of the PDFs $P_{a}\left(\right.$ green) and $P_{b}$ (blue) at the default parameter settings. The thinner lines show the mean plus or minus one standard deviation. Both the mean and standard deviation were estimated based on 1000 replicate runs. (b): Plot of $Q$ between the PDFs $P_{a}$ and $P_{b}$, as functions of time. The plot shows that on long time-scales the effects of adaptation increase. (c): Comparison between the earth-mover's distance $d_{e}$ (black), the Jensen-Shannon divergence $d_{J}$ (red), and the Euclidian distance $d_{E}$ (orange). To fit in the same graph, we plot $d_{e}$ divided by its maximimum value. For large values of $t, d_{J}$ and $d_{u}$ become insensitive to changes in the PDFs when there is no longer overlap between the PDFs. In contrast, $d_{e}$ continues to increase.

difference between PDFs, namely the Euclidian distance

$$
d_{u}=\sqrt{\sum_{j}\left|P_{a}(j) P_{b}(j)\right|^{2}}
$$

and the Jensen-Shannon divergence

$$
d_{J}=\frac{1}{2} \sum_{j}\left[P_{a}(j) \ln \left(\frac{2 P_{a}(j)}{P_{a}(j)+P_{b}(j)}\right)+P_{b}(j) \ln \left(\frac{2 P_{b}(j)}{P_{a}(j)+P_{b}(j)}\right)\right] .
$$

Similar to $d_{e}$ both measures initially measure an increasing difference between the PDFs (Fig 5.7c). For larger values of $t$ this increase flattens off because the measures reach a maximum when there is no overlap between the PDFs. The earth mover's distance $d_{e}$, in contrast, continues to increase because the distance between the means of the PDFs is still increasing.

\subsubsection{Resilience}

As shown in Ten Broeke, Van Voorn and Ligtenberg (2016), the model contains tipping points where the population collapses and goes extinct. For example, Fig 5.8 shows that a tipping point is crossed by decreasing the harvest parameter $E_{h}$. Low values of $E_{h}$ enable agents to obtain more energy within the same time interval and procreate faster. This leads to oscillations in the population size, as the population 
rapidly increases beyond what the environment can support. As $E_{h}$ decreases, the amplitude of these oscillations increases, eventually destabilising the system and causing extinction. In the following we consider a parameter change in the value of $E_{h}$ as a pressure to the system and we consider the resilience of the system against this pressure, and how this resilience is affected by adaptation.

To examine the long-term model behaviour for various values of $E_{h}$, we approximate time-averaged PDFs based on a single run of both versions of the ABM. The results for a few selected values of $E_{h}$ are shown in Fig 5.8. For $E_{h}=0.1$, both PDFs are bimodal, with the peaks corresponding to the extrema of the oscillations (Fig 5.8a). The distance between the peaks is smaller in the adaptive ABM than in the ABM without adaptation. For $E_{h}=0.05$, both ABMs converge to extinction, as indicated by the peaks at $n=0$ (Fig 5.8c). For $E_{h}=0.07$, extinction has occurred only in the ABM without adaptation. The adaptive ABM still has a positive population at the end of the run (Fig 5.8b).

We use 1000 replicate runs of both ABMs to approximate the PDFs as a function of time. The resulting histogram of the ABM without adaptation has a peak at $n=0$ and the height of the peak increases over time (Fig 5.9a). Thus, the probability that a population goes extinct remains positive over time, resulting in an increasing number of extinct runs as time progresses. The ABM with adaptation also has a peak at $n=0$, but the height of this peak is smaller and does not increase after the first few-hundred time-steps (Fig 5.9b). Thus, initially there is a positive probability of going extinct, but this probability decreases to zero over time. After some time, no more runs go to extinction. The adaptation measure $Q$ increases, since the number of runs resulting in extinction increases in the ABM without adaptation (Fig $5.9 \mathrm{c}$ ). Around $t=50,000$, all the model runs of the ABM without adaptation have gone to extinction, after which the adaptation measure no longer varies.

The variation between replicates in the time until the model goes to extinction is caused by stochasticity. To estimate the spread of this timing, we perform 100 model runs for each value of $E_{h}$ and record the value of $t$ where extinction occurs. For $E_{h}=0.07$, the number of runs of the ABM without adaptation with a positive population decreases approximately exponentially over time, whereas for the adaptive ABM extinction occurs only in the first few-hundred time-steps (Fig 5.10a). If the population does not go extinct during this initial period, then the population will adapt to harvest more often (Fig 5.10b), and move less often (Fig 5.10c). These adaptations ensure the long-term survival of the population.

To quantify the timing of the tipping point, we fit an exponential decay function to the number of model runs with a positive population at time $t$,

$$
N_{\text {pos }}(t)=N_{\text {pos }, 0} e^{-\lambda t}
$$

Here $N_{\text {pos }}(t)$ is the number of model runs with a positive population at time $t, N_{\text {pos }, 0}$ is the total number of model runs and $\lambda$ represents the rate of extinction. High values of $\lambda$ indicate that model runs rapidly go extinct, whereas $\lambda=0$ indicates that extinction does not occur at all. We estimate $\lambda$ by fitting Eq 5.9 to the simulation output using ordinary least squares. The values of the mean squared error of the fits show that Eq 5.9 gives a good approximation of the simulation output (Table 5.2). Fig 5.11 shows that $\lambda$ decreases as $E_{h}$ increases. Thus, extinction occurs faster for lower values of $E_{h}$. For all values of $E_{h}$, the fitted value of $\lambda$ is higher in the ABM without 


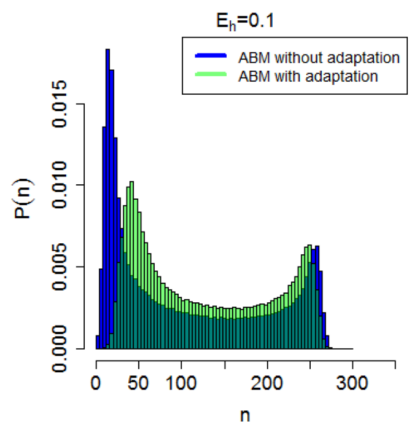

(a)

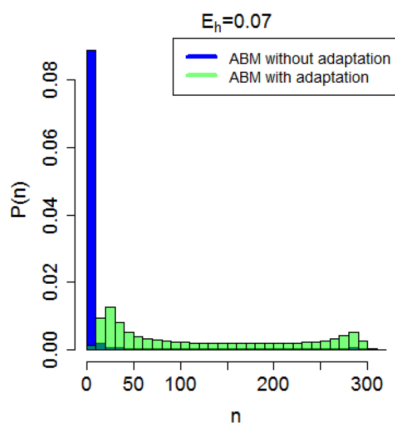

(b)

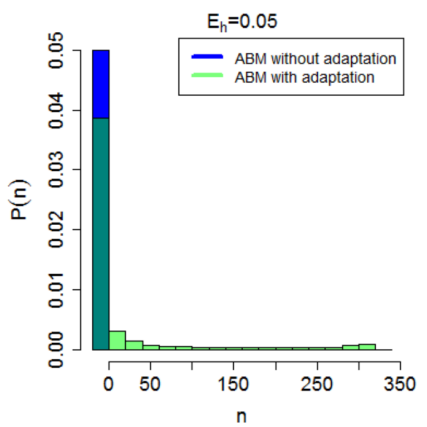

(c)

Figure 5.8: Time-averaged histograms of the number of agents $n$, for selected values of $E_{h}$.

The peaks at $n=0$ correspond to the population going extinct.

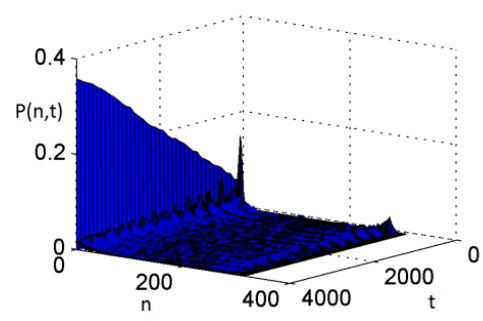

(a)

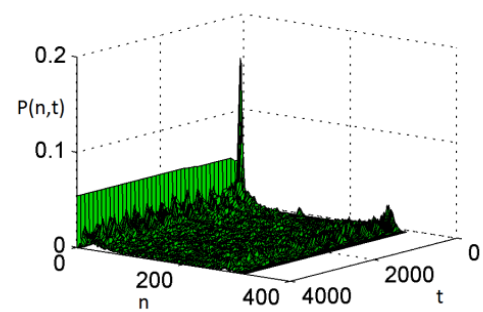

(b)

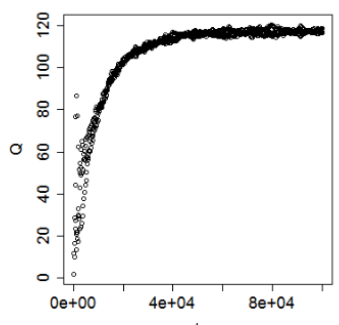

(c)

Figure 5.9: Time-dependent histograms at $E_{h}=0.07$. (a): Histogram of the ABM without adaptation. (b): Histogram of the ABM with adaptation. (c): The value of $Q$ as a function of time. The value of $Q$ increases as without adaptation an increasing number of runs goes to extinction.

adaptation compared to the ABM with adaptation, indicating that adaptation slows down extinction. We consistently observe that the tipping point where $\lambda$ goes to zero occurs at a lower value of $E_{h}$ for the ABM with adaptation than for the ABM without adaptation. For example, at $E_{h}=0.07$, the ABM without adaptation has a positive value of $\lambda$, indicating that all model runs eventually go extinct. On the other hand, in the ABM with adaptation populations survive on long time-scales. Our results thus show that the capacity of the agent population to adapt to its surroundings increases its resilience to circumstances that put pressure on the population.

Table 5.2: Values of the mean squared error (MSE) of the exponential fits to the number of replicate runs with surviving populations (Eq 5.9).

\begin{tabular}{|l|l|l|l|l|l|l|l|l|l|l|l|}
\hline$E_{h}$ & 0.0 & .01 & .02 & .03 & .04 & .05 & .06 & .07 & .08 & .09 & .10 \\
\hline Adaptation & 2.2 & 2.0 & 2.9 & 2.4 & 4.9 & 3.2 & 3.2 & 1.9 & 1.3 & 4.6 & 3.5 \\
\hline No adaptation & 5.7 & 3.3 & 1.6 & 1.8 & 2.1 & 5.8 & 5.2 & 9.5 & 3.2 & 7.5 & 3.5 \\
\hline
\end{tabular}




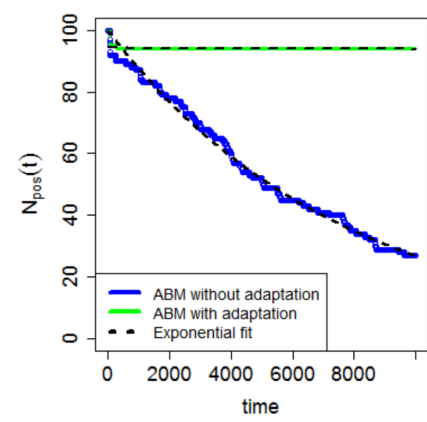

(a)

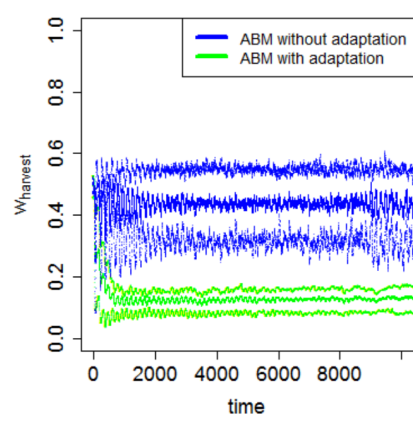

(b)

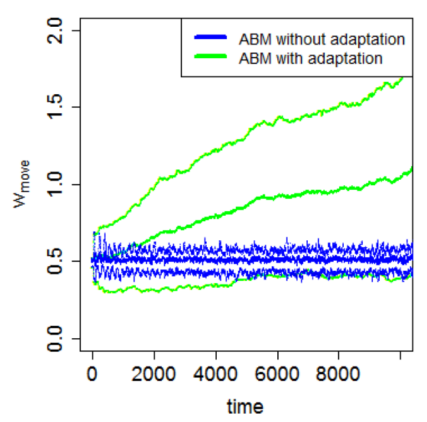

(c)

Figure 5.10: (a): Plot of the percentage of model runs with a positive population $N_{\text {pos }}(t)$ as a function of time. We used 100 replicate runs at $E_{h}=0.07$, for both versions of the ABM. The dashed lines correspond to exponential fits (Eq 5.9). Without adaptation, an increasing number of runs go to extinction, whereas with adaptation there is extinction only on short time-scales. (b): Mean value of $w_{\text {harvest }}$ over the agents of all the 100 replicate runs of the ABMs with adaptation and without adaptation. The upper and lower lines show the mean plus or minus one standard deviation. (c): The mean value of $w_{\text {move }}$ over the agents of all the 100 replicate runs of the ABMs with and without adaptation. The upper and lower lines show the mean plus or minus one standard deviation.

\subsubsection{Increasing pressure over time}

So far, we have considered the effect of adaptation on the ability of the population to cope with a pressure that is constant over time. In the following we will consider a pressure that increases over time, in the form of a gradual decrease in the diffusioncoefficient $D$. For high values of $D$ the resource spreads quickly from high density areas to low density areas, whereas for low values this spread is slow. Decreasing values of $D$ put pressure on the agent population, because agents need to search more actively for resource. Thus, we introduce a change in the value of $D$ over time,

$$
\rho=-\frac{d D}{d t}
$$

For positive values of $\rho$, the diffusion of the resource gradually slows down during a simulation run, putting pressure on the population. The value of $\rho$ determines the rate at which the pressure increases. We expect that if the pressure increases at a high rate, then adaptation must also occur at a high rate for the system to remain resilient. In the model, this rate of adaptation is determined by the random deviation of the agent characteristics upon reproduction. If these deviations are small, then offspring will be very similar to their parents and adaptation will be slow. If the deviations are larger, the differences between offspring and their parents are larger, which enables faster adaptation. The model parameter $z$ controls the size of these deviations. We examine the interaction between the rate of pressure increase and the rate of adaptation by running the model for different combinations of $\rho$ and $z$. Each run is initiated with parameter values of $D=0.1$, the energy cost of moving 


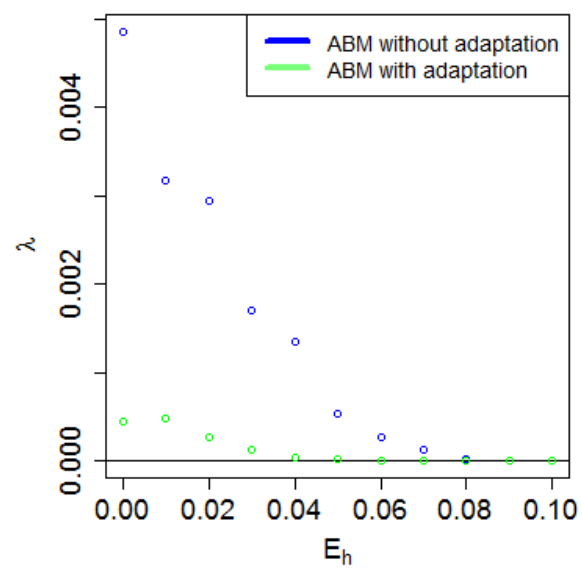

Figure 5.11: The extinction parameter $\lambda(\mathrm{Eq} 5.9)$ as function of the harvest cost $E_{h}$. The values were estimated by fitting Eq 5.9 to the output of 100 replicate runs of the ABMs with adaptation and without adaptation. The value of $E_{h}$ where $\lambda$ reaches zero corresponds to the tipping point where there is no extinction on long time-scales.

$E_{m}=0.1$ and $w_{\text {harvest }}=w_{\text {move }}=10$ for all agents. All other parameters are at the nominal setting. During each run, the pressure is increased by lowering the value of $D$ from $D=0.1$ to until $D=0.05$. Lower values of $D$ lead to extinction regardless of adaptations in the population. If adaptation is slow, then the pressure that results from the decrease of $D$ leads to collapse of the population, but if the adaptation is sufficiently fast then a positive population is maintained (Fig 5.12a). The mean value of $w_{\text {move }}$ across this population shows that agents adapt to move more frequently in search of resource, but that this adaptation is not fast enough for low values of $z$ (Fig 5.12b). Runs for various values of $z$ and $\rho$ show that there is a critical transition where the pressure on the population increases too fast for adaptations to keep up (Fig 5.12c). To better understand the resilience of the system to pressure, it is thus important to weigh the rate at which the population is able to adapt against the rate of changes that put pressure on the system.

\subsection{Conclusions \& Discussion}

For ABMs to be a useful tool for the assessment of the resilience of social-ecological systems, suitable methodologies for analysing these ABMs are needed. In this paper, we have proposed a methodology for analysing effects of agent adaptation in ABMs, and showed how this adaptation affects resilience. We have illustrated the use of this methodology by applying it to an ABM of consumers competing for a common-pool resource. The method is based on a comparison between the time-averaged PDFs of an adaptive $\mathrm{ABM}$ and version of this $\mathrm{ABM}$ for which adaptation has been disabled. We consider the difference between these two PDFs as a measure for the effects of 


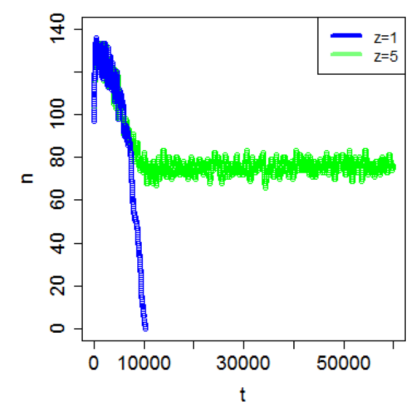

(a)

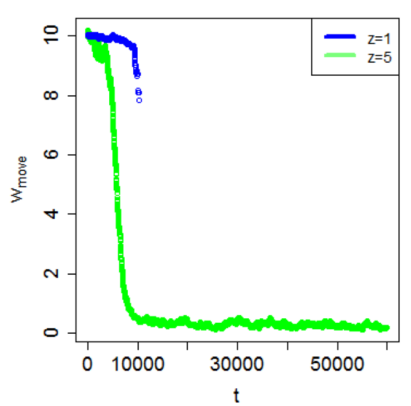

(b)

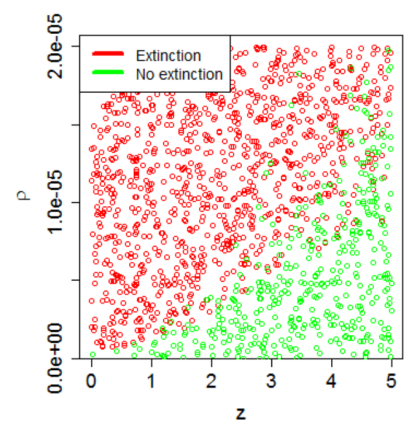

(c)

Figure 5.12: (a): Number of agents $n$ as function of time for a rate of adaptation $z=1$ (blue) and $z=5$ (green). In both plots $\rho=5 \times 10^{-5} \mathrm{Eq}$ (5.10). (b): Average value of $w_{\text {move }}$ across agents, for the same model runs as in (a). (c): Each point in the figure corresponds to a run of 60,000 time-steps. Red points indicate that the population has gone extinct, whereas for the green points the population has remained positive until the end of the simulation. Extinction occurs if the rate of adaptation $z$ is insufficient to cope with the pressure increase $\rho$.

adaptation on the model output. This difference is quantified using the earth-mover's distance Rubner et al. (1998) as a measure for the adaptation of the system. This measure differs from previously used sensitivity measures based on PDFs (e.g. Liu et al. (2006), Auder and Iooss (2008), Borgonovo (2007)) in that even when different model runs do not overlap in terms of output the earth-mover's distance is still able to quantify the sensitivity. Note that sensitivity measures quantify the output change or variation as a function of changes or variations in parameter values. Although Eq 5.1 measures the 'sensitivity' of the output to adaptation, it is not a sensitivity measure in the conventional sense because it does not take into account the size of the parameter change. When considering the sensitivity to a continuous parameter, the derivative of Eq 5.1 with respect to the parameter could be considered as a sensitivity measure. An advantage of the described method over most sensitivity analysis methods is that we explicitly consider the presence of tipping points, and how these tipping points are affected by adaptation. Existing sensitivity analysis methods do not take into account that the model behaviour may be qualitatively different in different regions of parameter space.

To test the potential of the methodology, we have applied it to a test-case ABM of agents competing for a common-pool resource. For this test-case, the methodology shows that adaptation increases the resilience of the system to pressures. This resilience is defined in terms of the amount of pressure the system can cope with before it jumps to an alternative domain of attraction. In our case, this jump corresponds to the tipping point where the agent population goes extinct. Without adaptation, this tipping points always occurs at certain parameter values. Agent adaptation is added in the form of a process of natural selection. As a result of this adaptation, the location of the tipping point shifts, and the boundaries of the domain of attraction become wider. This positive contribution of adaptation to the resilience of the testcase depends on the rate at which adaptation takes place. The analysis of the ABM 
shows that for a system to be resilient, the rate of adaptation needs to be sufficiently fast to cope with increasing pressures or changing conditions.

Other authors have discussed ABMs similar to our test-case Burtsev and Turchin (2006), Aubert-Kato et al. (2015), Colon et al. (2015) In Burtsev and Turchin (2006), Aubert-Kato et al. (2015) the focus is mostly on the evolution of behavioural strategies for the agents, which are characterised by a neural net. Both studies find that various strategies evolve, ranging from greedy to cooperative. In our ABM the range of possible strategies is smaller, because the ABM contains only two agents parameters that are affected by natural selection. For any given parameter setting the model seems to converge to a dominant strategy, but it depends on the parameter setting what this strategy is. For some settings it corresponds to 'greedy' agents that harvest as often as possible, whereas for other settings it corresponds to agents that harvest less frequently in order to let the resource grow. A possible extension to our model would be to include more agent parameters, possibly involving direct interactions between agents such as in Burtsev and Turchin (2006). For the present study, our main aim is to examine how agent behaviour affects the resilience of the system as a whole. In Colon et al. (2015) the resilience of different stability domains of an ABM is considered, but here the agent behaviour is completely pre-defined, and the system does not contain adaptation.

In this paper we have considered only adaptation through a process of natural selection. Adaptation through natural selection typically takes place on long timescales relative to other types of adaptation. Furthermore, adaptation through natural selection is reactive in the sense that it that responds to changes in the environment, but cannot respond to changes that agents might foresee in the future. In many CAS, individual agents have the ability to learn from past experiences and to adjust their behaviour according to expected future developments. These types of adaptation can be expected to operate on much shorter time-scales than natural selection Gell-Mann (1994). Future work will include investigating types of adaptation that operate on faster time-scales, or in which agents adapt proactively based on foreseen changes. Such fast adaptations might lead to an increase in the resilience of the system as agents are able to quickly adapt to situations and respond to pressures. Alternatively, however, they might also destabilise the system, for example when agents mispredict trends or overspecialise Nelson (2011)).

In this paper, we have analysed the effects of adaptation in a simulated CAS. The analysis is based on a comparison between a non-adaptive and an adaptive ABM. In order to apply this methodology to a real-life CAS, one thus needs to develop model versions with and without adaptation. To this end, one needs to assess whether the system is adaptive, and what system components and interactions are relevant to understanding this adaptation. Qualitative tools for this kind of assessment are available (e.g. Alliance (2010)). The methodology in this paper complements such resilience assessments by quantifying the resilience of the system using ABMs.

Our results imply that for studies that aim to assess or to enhance the resilience of social-ecological systems, it is relevant to consider the ability of the system to adapt. When combined with suitable methodologies for sensitivity analysis, ABMs can be a helpful tool to test hypotheses on which factors may contribute to the resilience of social-ecological systems (e.g., Heckbert et al. (2014), Janssen and Carpenter (1999), Schlüter and Pahl-Wostl (2007), Schouten et al. (2013)). 


\section{Acknowledgments}

We thank the people in the Wageningen University IP/OP CAS theme for valuable discussions.

\section{A Stationarity and ergodicity tests}

\section{Stationarity and ergodicity tests.}

For some ABMs stationarity and ergodicity can be proven analytically, without the use of simulation runs (Izquierdo et al. 2009). For many ABMs, however, such a formal proof is not possible because we do not have an analytical description of the processes that generate the output (Grazzini 2012). We then have to rely instead on statistical tests for stationarity and ergodicity of the output of simulation runs. Such tests cannot formally prove stationarity or ergodicity, but may statistically verify these properties. A number of statistical tests are available for this purpose (Phillips and Xiao 1998). Most of these tests are parametric tests that require assumptions on the shape of the output PDF, such as the assumption that the output is normally distributed. For ABMs analysis, however, the complexity of the model often makes it difficult to predict the shape of the output PDF (Grazzini 2012). Furthermore, since an investigation of the stationarity is often one of the first steps in model analysis, we may not have investigated the properties of the output PDF before we test the stationarity. In this paper we therefore use only non-parametric tests, which impose no assumptions on the output PDF. The downside of using parametric tests is that they are relatively conservative, i.e. using these tests it is difficult to reject the nullhypothesis that the series is stationary. In the context of analysing data from ABMs, this limitation may be partially addressed by increasing the amount of data that is recorded from the ABM, or by adapting the used significance level. To test the stationarity we first divide the time-series into a number of short-term time-windows and compute the average of the model output over each window (Grazzini 2012). By averaging over time-windows we aim to remove short-term fluctuations from the timeseries. Removing these fluctuations ensures that the window-means can be considered as independent observations of the stochastic process. The stationarity test then tests for the presence of trends in this series of observations. Two commonly used non-parametric tests are the Runs Test (Wald and Wolfowitz 1940, Grazzini 2012, Organization 2006) and the Mann-Kendall trend test (Mann 1945, Kendall 1938, Bendat and Piersol 2011, Yue et al. 2002, Organization 2006). The trend test is relatively powerful for the detection of monotonic trends, whereas the runs test is typically more powerful for the detection of fluctuating trends (Bendat and Piersol 2011). We therefore use both tests to verify and supplement each other. The details of both tests will follow below. To verify ergodicity of the model output we use the same tests, but applied to different samples (Grazzini 2012). The first sample is composed of a single model run, divided into a number of equal length subseries. The second sample is composed of a number of model runs, with the length of each run equal to the length of a subseries of the first sample. We now use the runs test or trend test to compare the means of the subseries of the first sample to the means of the model runs of the second sample. If the model is ergodic, then the two sets of means come from the same PDF. Thus, with the ergodicity test we test the null-hypothesis that 
the underlying PDFs are equal.

\section{A.1 Runs Test}

To test stationarity to of a time-series, we apply the Runs test with the null-hypothesis that the series is stationary, i.e. the underlying distribution does not change over time. The Runs test considers for each observation in the time-series whether the observation is smaller than, or larger than the mean $E\left(n_{t}\right)$ over the entire time-series. Based on this comparison, we compute the vector $H_{t}$ so that

$$
H_{t}= \begin{cases}0 & \text { if } n_{t}<E\left(n_{t}\right) \\ 1 & \text { if } n_{t}>E\left(n_{t}\right)\end{cases}
$$

where we ignore observations that are equal to the mean. If the output is stationary we expect the deviations from the mean to be randomly distributed over time. If, however, there is a trend then the deviations are not randomly distributed. For example, for an upwards trend $H_{t}$ will have more 0 's for smaller $t$ and more 1 's for larger $t$. Such irregularities in $H_{t}$ are expressed in terms of the number of "runs" $R$. A run is defined as a consecutive series of identical symbols in the vector $H_{t}$. For example, the series $0,1,0,0,0,1,1,0$ contains 5 runs $(0,1,0,0,0,1,1,0)$. If the time-series is stationary, then the expectation value and the variance of the random variable $\mathrm{R}$ are known (Wald and Wolfowitz 1940). For the mean we have

$$
E(R)=\frac{2 N_{+} N_{-}}{N_{+}+N_{-}}+1
$$

with $N_{+}$the number of observations above the total mean and $N_{-}$the number of observations below the total mean. The variance is

$$
V(R)=\frac{2 N_{+} N_{-}\left(2 N_{+} N_{-}-N_{+}-N_{-}\right)}{\left(N_{+}+N_{-}\right)^{2}\left(N_{+}+N_{-}-1\right)}
$$

We can thus test for non-stationarity by comparing the value of $\mathrm{R}$ measured from a time-series to these equations, which give our null-hypothesis. If the number of runs differs significantly then we reject the null hypothesis that the output is stationary.

The value of $\mathrm{R}$ can be affected by dependency between observations that are close in time. For example, if an observation at time $t$ lies above the mean, then the observation at time $t+1$ may also tend to lie above the mean. The resulting number of runs would then be smaller than would be expected based on the above equations. The dependency between observations is removed by averaging the observations over time-windows. A single observation then corresponds to the mean of a window and the vector $H_{t}$ is computed over these window means.

\section{A.2 Trend Test}

The trend test is a commonly used alternative to the runs test for establishing stationarity of a stochastic process (Bendat and Piersol 2011, Organization 2006). The trend test is more powerful for finding monotonic (upwards or downwards) trends, whereas the runs test is better at finding fluctuating trends (Bendat and Piersol 2011). We 
will thus use both tests to verify and complement each other. As in the runs test, the null-hypothesis is that the underlying distribution of a time-series is stationary. The trend test is based on the value of,

$$
W=\sum_{t=1}^{N_{m}-1} \sum_{t^{\prime}=t+1}^{N_{m}} \operatorname{sgn}\left(n_{t}-n_{t}^{\prime}\right)
$$

where $T$ is the number of observations and

$$
\operatorname{sgn}(n)= \begin{cases}-1 & \text { if } n<0 \\ 0 & \text { if } n=0 \\ 1 & \text { if } n>0\end{cases}
$$

For a sufficiently long time-series, $W$ is normally distributed with mean and variance,

$$
\begin{aligned}
& E(W)=0 \\
& V(W)=\frac{1}{18} N_{m}\left(N_{m}-1\right)\left(2 N_{m}+5\right)-\sum_{g} g(g-1)(2 g+5)
\end{aligned}
$$

Here the summation $\sum_{g}$ runs over all groups of observations that have tied output values $n$ and $g$ is the number of measurements in each of these groups. These equations for the mean and variance describe the null-hypothesis that the actual values of $\mathrm{W}$ are tested against. Similar to the runs Test, the trend is affected when there is a dependency between observations that are close in time. We may divide the series into time windows and treat the window averages as individual observations to remove such dependencies.

\section{B Computation of the earth mover's distance.}

Computing the earth-mover's distance between a pair of distributions amounts to finding the minimal 'work' needed to change on distribution into the other. In this paper, we will deal with pairs of one-dimensional discrete output distributions, $P_{a}(n)$ and $P_{b}(n)$, where we have dropped the time-dependency for convenience. The earthmover's distance is then written as (Ling and Okada 2007),

$$
d_{e}=\min \left(\sum_{(j, k)} g(j, k) d(j, k)\right)
$$

with the constraints,

$$
\begin{aligned}
\sum_{k} g(j, k) & =P_{a}(j) \forall j \\
\sum_{j} g(j, k) & =P_{b}(k) \forall k \\
g(j, k) & \geq 0 \forall j, k
\end{aligned}
$$


with $g(j, k)$ the flow between output values, $d(j, k)$ the distance between output values, and the indices $\mathrm{j}$ and $\mathrm{k}$ running over all possible output values. The first two constraints ensure that the flow is such that the distribution $P_{a}(j)$ is transformed into $P_{b}(k)$. The third constraint ensures that mass is moved from $P_{a}(j)$ to $P_{b}(k)$, and not the other way around. The first equation ensures that the flow is chosen such to minimise the required 'work' $g(j, k) d(j, k)$. For two PDFs of a single output variable, this minimisation is accomplished by going through all consecutive pairs of output values, and keeping track of the amount of mass that needs to be transported, such as in the following piece of pseudo-code.

$$
\begin{aligned}
& u(0)=0 \\
& \text { for }\left(i=1: N_{b}\right) \\
& \qquad u(i)=u(i-1)+P_{a}(i)-P_{b}(i) \\
& \text { end } \\
& d_{e}=\sum_{i}|u(i)|
\end{aligned}
$$

Here the vector $u(i)$ stores the amount of mass that needs to be transported between consecutive bins, and $N_{b}$ is the total number of bins.

\section{C Results of stationarity and ergodicity tests.}

\section{C.1 ABM without adaptation}

We investigate the convergence of the output of the ABM without adaptation to a stationary state in the nominal setting. The mean of the model output $n$ over 10,000 replicates as a function of time (Figure 5.13a) shows that the output initially oscillates, but stabilizes before $t=1000$, after which it shows minor fluctuations around a mean value. The estimated output PDFs at $t=1000$ and $t=2000$ are approximately equal (Figure 5.13b) and the model thus seems to be stationary. We further verify stationarity by performing a runs test (Grazzini 2012) and a trend test (Bendat and Piersol 2011, Organization 2006) between $t=1000$ and $t=2000$ on each of the separate 1000 model runs. We use a 5\% significance level. If the model is stationary we thus expect that the null-hypothesis will be rejected for $5 \%$ of the model runs. If we treat each time-step as an independent observation, then the null-hypothesis is rejected for most model runs. These rejections occur because the observations at times $t$ and $t-1$ are not independent, but related through the processes of birth and death. This dependence is revealed by plots of the autocorrelation function (ACF) and the partial autocorrelation function (PACF). The ACF is the correlation of the time series with itself at different lags in time. Figure 5.14a shows that the ACF decreases only slowly as the lag is increased. Even at a lag of 100 time-steps, there is a significant autocorrelation. The PACF is the correlation of the time series with itself at different lags in time, that is not explained by correlations at smaller lags. The PACF shows a strong peak at a lag of 1 time-step, but is not significant at a lag of two time-steps (Figure 5.14b). From this finding we can conclude that the correlation between larger lags is caused by the correlation at lag 1 . To test the stationarity, we remove the dependence between observations by dividing the series into a number of 


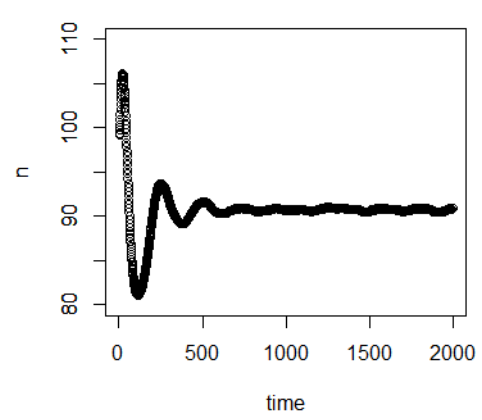

(a)

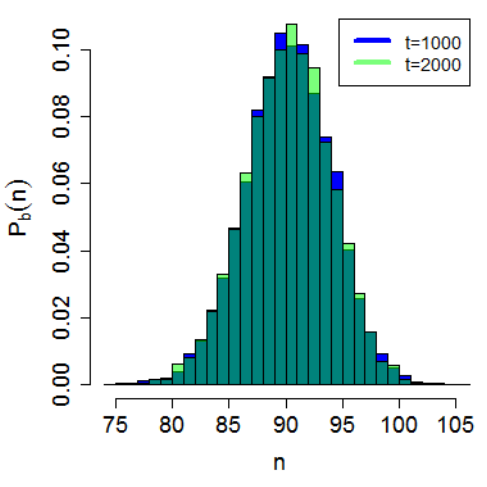

(b)

Figure 5.13: (a): The model output $n$ of the ABM without adaptation, averaged over 1000 replicate runs in the nominal parameter setting as function of time. (b): Histograms of the ABM without adaptation at $t=1000$ (blue) and at $t=2000$ (green).

equal length windows. If the windows are sufficiently long, then the window means of the output can be considered as independent observations of the output. The rejection rate then converges to around $5 \%$, indicating that the model is stationary (Figure 5.15). The trend test requires a shorter window length for this convergence than the runs test. This is consistent with the finding of Bendat and Piersol (2011) that the trend test tends to be less sensitive to fluctuating trends than the runs test. We conclude from Figures 5.13 amd 5.15 that the model output is stationary between $t=1000$ and $t=2000$.

For the ergodicity test we compare the output of a single run measured over a long time to the output of a large number of replicate runs, measured at a set time. The PDF of a single model run of 100,000 time-steps is approximately equal to the PDF of 10,000 model runs at $t=1000$ (Figure5.16a). This indicates that the model is ergodic. To further verify ergodicity we follow the procedure of (Grazzini 2012). We divide the long model run into 100 subseries of 1000 time-steps. The means of these subseries compose the first sample of the ergodicity test. The second sample consists of 1000 model runs, each averaged between $t=1000$ and $t=2000$. A visual comparison between the two samples is shown in Figure 5.16b. A runs test does not reject the null-hypothesis that the two samples come from the same distribution. Based on this test and the visual comparison of the PDFs, we conclude that the model is ergodic.

\section{C.2 Adaptive ABM}

For the adaptive ABM, the results of the stationarity test are similar to the case without adaptation. The model output stabilises before $t=1000$ and does not seem to significantly change between $t=1000$ and $t=2000$ (Figure 5.17). A runs test and trend test for stationarity confirm that the output is stationary, as long as the 


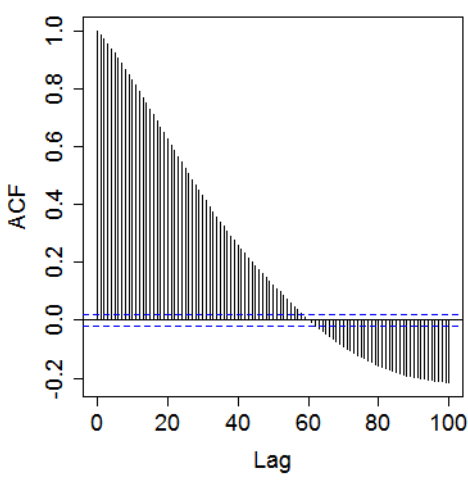

(a)

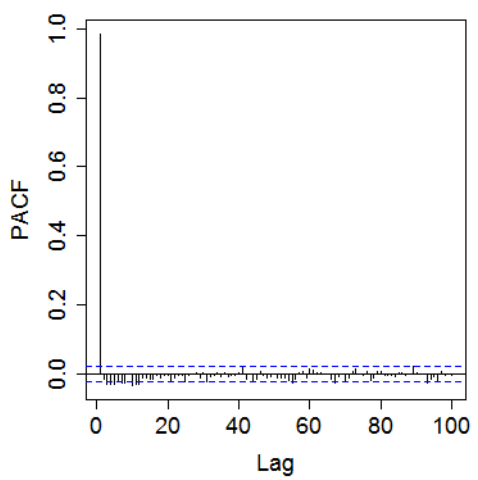

(b)

Figure 5.14: (a): Plot of the ACF as function of the lag. The dashed blue lines show the $5 \%$ confidence interval. (b): Plot of the PACF as function of the lag. The dashed blue lines show the $5 \%$ confidence interval.

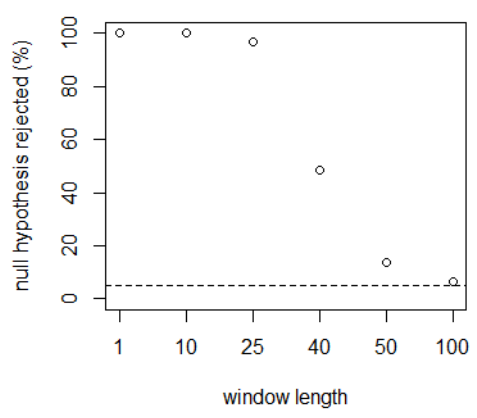

(a)

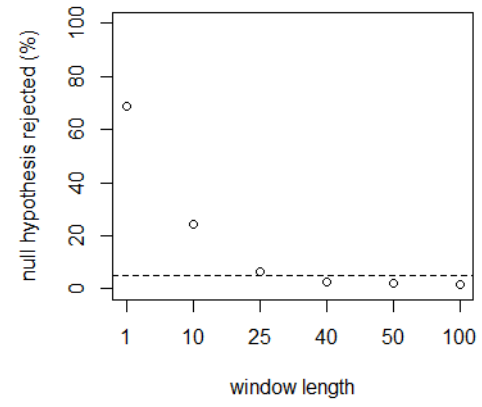

(b)

Figure 5.15: (a): Percentage of replicate runs for which the runs test rejects the nullhypothesis of stationarity for the ABM without adaptation. On the horizontal axis is the window length, i.e. the number of time-steps over which the output is averaged. The dashed line indicates the 5\% significance level. (b): Same as (a), but using the trend test instead of the runs test. 


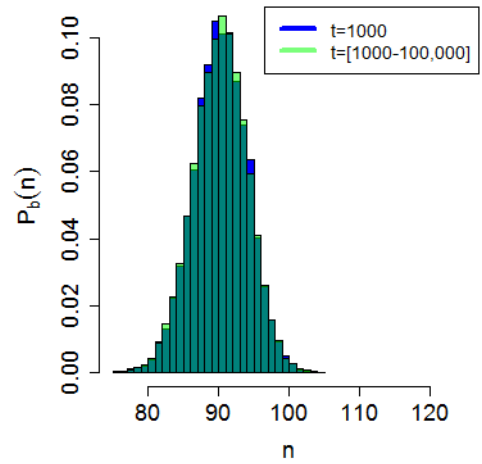

(a)

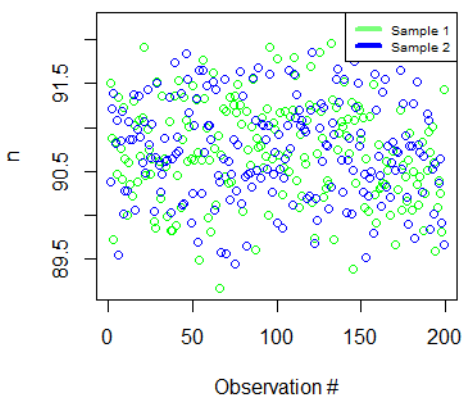

(b)

Figure 5.16: (a): Histograms of the model output of the ABM without adaptation. The blue histogram is measured over 10,000 replicate runs at $t=1000$, and the green histogram over the time-steps of a single model run between $t=1000$ and $t=100,000$. (b): Samples used for the runs test for ergodicity of the ABM without adaptation. The green sample points are the averaged output of a single model run over a window of 50 time-steps. The blue sample points each correspond to a different model run, averaged between $t=1000$ and $t=2000$.

window length is sufficiently long to remove short term fluctuations from the output (Figure 5.18). The model thus appears to be stationary between $t=1000$ and $t=$ 2000.

We use an ergodicity test to explore the long-term model behaviour. The adaptive ABM is not ergodic. The PDF of a long model run measured over time clearly differs from the PDF at $t=1000$ (Figure 5.19). Over time, the population gradually adapts, which leads to an increase in the population size. The trend test shows that there is a positive trend in the adaptive ABM. Based on the series of window means in Figure 5.19b, we used the Theil Sen method to estimate the slope. This yields a slope of $1.20 * 10^{-4}$ agents per time-step. 


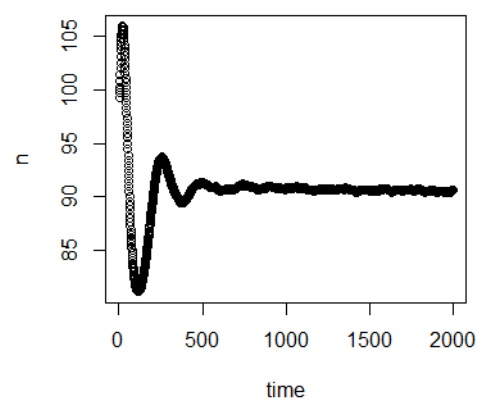

(a)

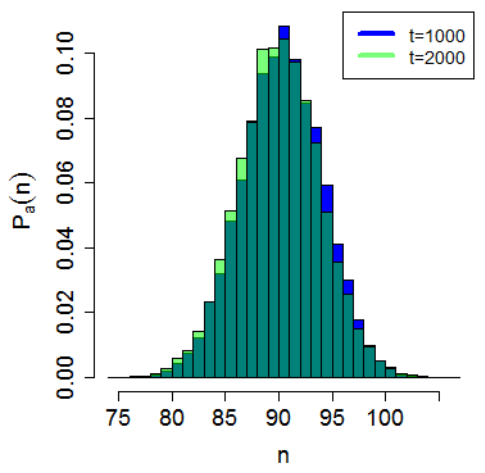

(b)

Figure 5.17: (a): The model output $n$ of the ABM with adaptation, averaged over 1000 replicate runs in the nominal parameter setting as function of time. (b): Histograms of the ABM with adaptation at $t=1000$ (blue) and at $t=2000$ (green).

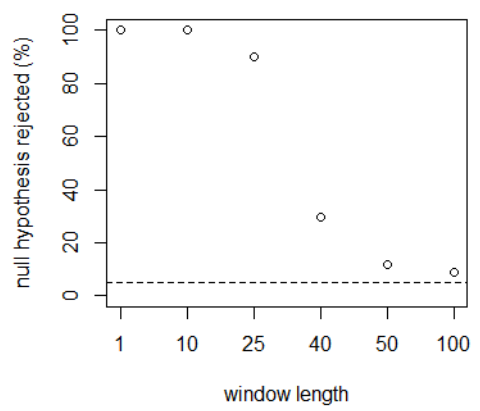

(a)

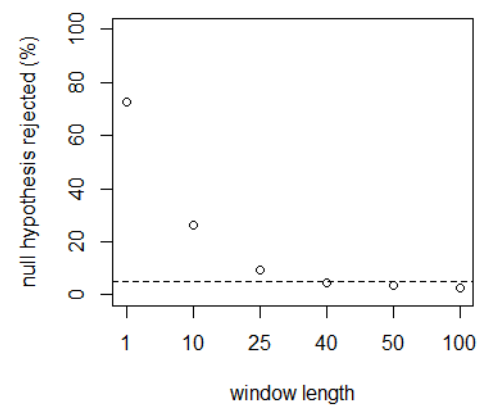

(b)

Figure 5.18: (a): Percentage of replicate runs for which the runs test rejects the nullhypothesis of stationarity for the ABM without adaptation. On the horizontal axis is the window length, i.e. the number of time-steps over which the output is averaged. The dashed line indicates the 5\% significance level. (b): Same as (a), but using the trend test instead of the runs test. 


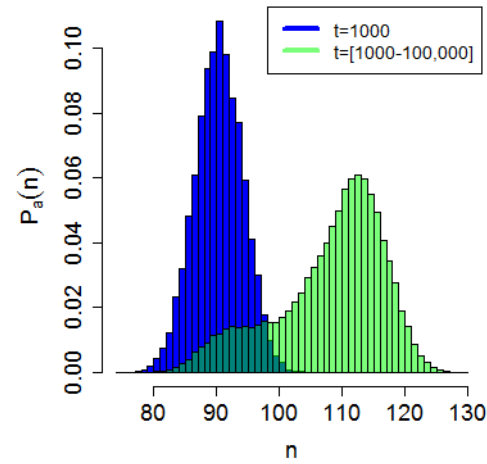

(a)

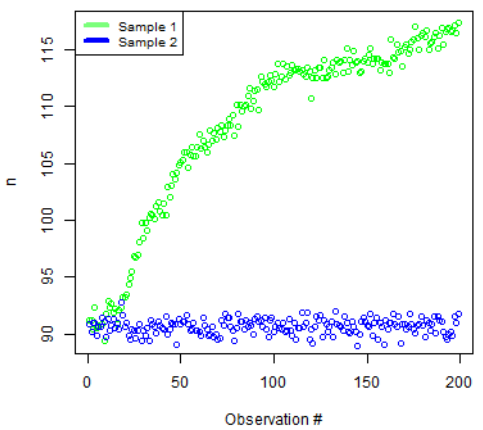

(b)

Figure 5.19: (a): Histograms of the model output of the ABM with adaptation. The blue histogram is measured over 10,000 replicate runs at $t=1000$, and the green histogram over the time-steps of a single model run between $t=1000$ and $t=100,000$. (b): Samples used for the runs test for ergodicity of the ABM with adaptation. The green sample points are the averaged output of a single model run over a window of 50 time-steps. The green sample points each correspond to a different model run, averaged between $t=1000$ and $t=2000$.

\section{D Default parameter setting}


Table 5.3: Default setting of model parameters

\begin{tabular}{|l|l|l|l|}
\hline Symbol & Description & Dimensions/range & Default value \\
$c$ & Efficiency & $\mathrm{J} \mathrm{kg}^{-1}$ & 0.9 \\
$D$ & Diffusion coefficient & $\mathrm{km}^{2} \mathrm{day}^{-1}$ & 0.1 \\
$E_{b}$ & Birth energy & $\mathrm{J}$ & 5 \\
$E_{h}$ & Harvest cost & $\mathrm{J}$ & 0.3 \\
$E_{m}$ & Cost of energy maintenance & $\mathrm{J}$ & 0.1 \\
$E_{\text {move }}$ & Move cost & $\mathrm{J}$ & 0.5 \\
$K$ & Carrying capacity & $\mathrm{kg}$ & 2 \\
$n_{0}$ & Initial number of agents & $0,1,2, \ldots$ & 100 \\
$r$ & Growth rate & $\mathrm{S}^{-1}$ & 0.1 \\
$R_{0}$ & Initial resource & $0-1($ proportion of $K)$ & 1 \\
$R_{\max }$ & Maximum harvest & $\mathrm{kg}$ & 0.5 \\
$R_{u n c}$ & Uncertainty of resource estimations & $\mathrm{kg}$ & 0.1 \\
$v_{b}$ & Birth coefficient & $\mathrm{J}^{-1}$ & 10 \\
$v_{d}$ & Mortality coefficient & $\mathrm{J}^{-1}$ & 10 \\
$z$ & Variation in offspring traits & {$[0, \rightarrow)$} & 0.2 \\
\hline
\end{tabular}




\section{Chapter 6}

\section{Cooperation can improve the resilience of common-pool resource systems against over-harvesting}

G.A. ten Broeke, G.A.K. van Voorn, A. Ligtenberg, J. Molenaar.

Under review. 
Currently common-pool resource systems world-wide are under pressure due to overexploitation and environmental change. To ensure that these systems continue to provide vital ecosystem services it may be necessary to increase their resilience against such pressure. One way of doing this may be to improve cooperation among agents who are heavily involved in common-pool resource systems, such as farmers, fishers, managers, and companies. Historical examples suggest that the persistence or collapse of common-pool resource systems may hinge on agents collaborating or not, but cooperation as a mechanism to improve resilience is not commonly included in existing models for studying resilience. Cooperation may be sustained through indirect reciprocity, i.e., cooperative behaviour by one agent may be repaid by other agents. In this paper we develop a suite of models to understand how cooperation can evolve, and how it affects the resilience of common-pool resource systems. This suite of models contains various mechanisms for indirect reciprocity. These mechanism are modelled as agent-based models (ABMs), which represent a generic spatial common-pool resource system. The results show that although indirect reciprocity positively affects the level of cooperation in the system, cooperation is common even without indirect reciprocity. Moreover, the presence of cooperation increases the resilience of the system against shocks that make it more difficult to gather resource. This resilience is assessed by applying various shocks in the form of changes to state variable or parameter values, and measuring whether the agent population can recover from these shocks, and how much time is needed for this recovery.

\subsection{Introduction}

The continuing increase in the world population and its associated increase in human activity and environmental effects puts severe pressure on many social-ecological systems (SES) (Cabell and Oelofse 2012). A particular risk seems to be a sudden loss of ecosystem services delivered by these SES through a breakdown in resilience followed by random shocks, for example, a climate event or large price fluctuation (Scheffer et al. 2012). Resilience is then defined as "the capacity of a system to absorb disturbance and reorganize while undergoing change so as to still retain essentially the same function, structure, identity, and feedbacks" (Walker et al. 2004); in this context esilience is the ability of SES to absorb shocks while maintaining the provision of ecosystem services (Folke et al. 2010). This resilience depends not only on biophysical factors, but also on social factors (Janssen 2015). Many SES, suh as fisheries (Basurto et al. 2013), irrigation systems (Cifdaloz et al. 2010), and grasslands (Crépin and Lindahl 2009), involve common-pool resources that are shared between multiple actors. In such common-pool resource systems, social norms and cooperation between actors may be important factors for the management of the resources (Janssen 2015, Ostrom et al. 1999, Ostrom 2015, Libre et al. 2015).

In economic theory, interactions between individuals in common-pool resource systems are commonly studied using game theory dilemmas, such as the well-known prisoner's dilemma (Kreps et al. 1982, Mao et al. 2017). These studies predict that under the assumption of economic rationality, individuals will over-exploit a resource to maximise their short-term personal profits. Eventually such over-exploitation will 
lead to a collapse of the system. This outcome is commonly known as the tragedy of the commons (Hardin 2009, Milinski et al. 2002). One well-known example of this is the collapse of the North-Atlantic cod fishery due to over-harvesting (Milich 1999).

Historical examples as well as more current studies suggest that cooperation may be an important mechanism through which a tragedy of the commons may be avoided, and the resilience of common-pool resource systems may be promoted (Ostrom et al. 1999). For example, in grasslands, which often are owned by several farmers, cooperation between farmers may be needed to avoid degradation due to over-grazing (Crépin and Lindahl 2009). Another example is the Dutch water control system, which has been developed and maintained through cooperation between large numbers of actors (Kaijser 2002). Yet, it has also been reported that under some circumstances cooperation may lead to such an efficient exploitation of resources, that it may cause over-harvesting (Crépin and Lindahl 2009). High levels of cooperation may lead to more efficient harvesting and an increased population size, which in turn may put pressure on resource availability. These kinds of feedbacks are especially important for common-pool resource problems, in which the collective actions of agents can change the entire system, for example through the creation of pollution, or the depletion of a finite resource (Gotts et al. 2003). Thus, whereas cooperation appears to be an important factor in the resilience of common-pool resource systems, its effects may be subtle and context-dependent. More cooperation will not always lead to a higher resilience.

In this paper we aim to study the effects of cooperation between agents on the resilience of common-pools resource systems against random shocks against a background of increased harvesting. In order to do so, we introduce a suite of models. In these models agents may harvest resource individually or cooperatively. The incentive to cooperate rather than defect is given by indirect reciprocity, which is based on reputation (Nowak and Sigmund 1998, Nowak 2006). An individual that cooperates will gain a good reputation, and in turn receive cooperation from others. Interacting agents however can also defect. Defection directly increases the gain for an agent at the cost of the other individual, but it also means that the reputation of the defecting agent may decrease, incurring a future cost.

A common way of modelling trade-off dynamics between direct benefits and indirect costs is by using game theory models. These models typically assume a constant population size and play out until some steady state value is obtained (Doebeli and Hauert 2005). The main assumption in game theory models is that agents are capable of decisions based on 'perfect' information and decision-making, i.e. they know exactly what options exist and what the expected costs and benefits associated with each of these options are because they are pre-defined (so-called 'utility' functions). This assumption may hold under certain conditions, but agents involved in many SES have limited information available and are subject to social rules and behaviour 'outside' economic rational theory. This is the case for instance in fisheries, a typical example of common-pool resource systems, where social norms affect decisions surrounding fishing and when to exit the fishery business (Libre et al. 2015). It is increasingly argued that social behaviour of agents should be considered when modelling SES (Schlueter et al. 2012). Hence, in this paper we use Agent-based Models (ABMs). These models are composed of autonomous agents that have behavioural rules for interacting with each other and their environment. In addition, these be- 
havioural rules may change over time as agents learn from previous experiences. The models also include dynamic interactions between the resource and the agents.

The remainder of the paper is set up as follows. Section 6.2 provides an overview of the models. In our ABMs the costs and rewards depend on resource availability. Several mechanisms for cooperation are distinguished between the models, namely reputation may $1 /$ influence the decision of which individuals to interact with, 2 / influence the decision of whether to accept an interaction or harvest individually, or $3 /$ influence the decision of whether to defect or cooperate. In Section 6.3 we discuss the output measures that are collected from the model, and the methodologies that are used to analyse the model results. For the analysis of the ABM results we will use methodologies of sensitivity analysis to assess the resilience of the population against extinction (Ten Broeke, Van Voorn and Ligtenberg 2016). Section 6.4 contains the results of this analysis. In Section 6.5 we conclude with the discussion.

\subsection{Model Description}

To assess the effects of cooperation, we will use five different versions of an ABM in which alternative mechanisms for cooperation are implemented. A full description of the model mechanisms is given in the ODD format (Grimm et al. 2006, 2010) provided in 6.A. Here a summary is given, while the agent decision rules regarding cooperation and defection are discussed in more detail.

In the different model versions agents may harvest the common-pool resource either cooperatively, or individually. By harvesting cooperatively, agents may gain a higher yield. Different model version contain different mechanisms that can lead to cooperation. These mechanisms are shown in Fig. 6.1 (adapted from (Ostrom and Walker 2004)). Central to all of these mechanisms is trust between agents. Trust is then based on two factors, namely reputation and the memory of agents of previous interactions. Cooperation carries a risk, because interaction partners have the option to 'defect'. Defection gives a higher yield for the defector, at the cost of its interaction partner. In absence of any mechanisms to promote cooperation, selfish agents would maximise their profits by always defecting. The reputation of an agent is based on all its previous interactions. Agents that often cooperate will gain a good reputation, whereas agents that often defect will gain a bad reputation.

The five model versions used in the study are:

1. The basic model. It does not contain any mechanism to promote cooperation. Although agents have the option to cooperate, there is no incentive to do so. We use the basic model as a benchmark for comparison to the other model versions.

2. The reciprocity model. It is based on reciprocity. Agents tend to reciprocate previous behaviour of interaction partners. If an interaction partner is trusted, it is inferred that the partner gained this trust by cooperating in the past. Therefore, trusted agents are more likely to receive cooperation in return, whereas untrusted agents are more likely to suffer defection.

3. The partner choice model. Agents consider trust in their choice of which other agents to interact with. Trusted interaction partners are preferred over un- 
trusted ones. As a result, agents may lose future opportunities for interaction after defection.

4. The voluntary interaction model. Agents consider trust in their decision to harvest individually or cooperatively. If potential interaction partners are not trusted, agents are more likely to harvest individually instead of cooperatively. Again, this means that untrusted agents lose interaction opportunities.

5. The full model. In this version all three mechanisms are included. Thus, each agent considers trust in its decisions of whether to harvest individually or cooperatively, and which other agents to interact with. Furthermore, agents also vary in the extend to which they tend to reciprocate previous behaviour of other agents.

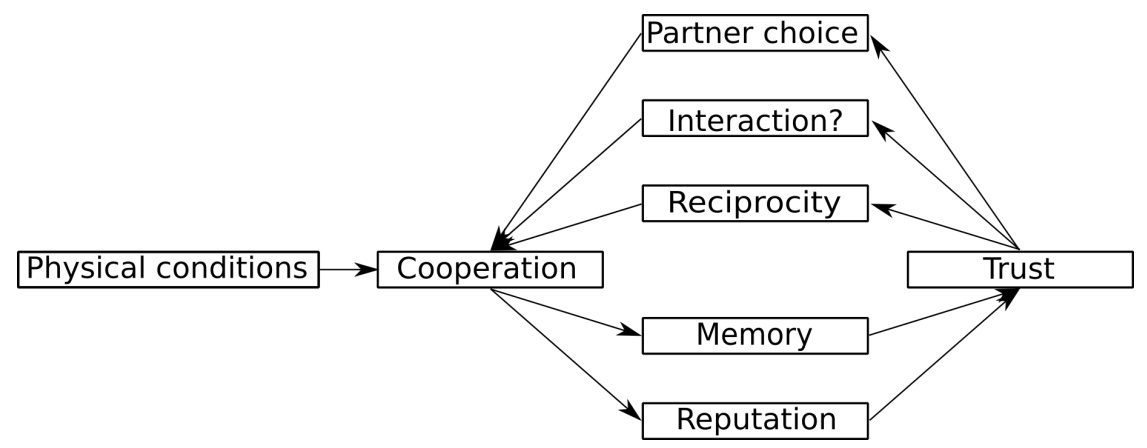

Figure 6.1: This flowchart shows the factors that affect the level of cooperation in the system. It has been adapted from Ostrom and Walker (2004), the most important change being that in our version cooperation is not only affected by trust, but also affects trust. A detailed explanation is given in the main text.

In the model it is assumed that recent interactions weigh more heavily than previous ones, and reputation is updated using an exponential decay function. The reputation of agents is observable for other agents, with a random observation error. Memories of previous interactions differ from reputation, because these memories are accessible only to the agents that participated in the interaction. Memory scores are assigned to each pair of agents that previously interacted. A high memory score indicates that the other agent often cooperated, whereas a low score indicates that it often defected. Like reputation, this memory score is updated using a decay function. Trust is computed as a weighted average of reputation, and the memory score. The relative weight between both factors is given by a parameter that varies between agents. If no memories of previous interactions are available, then the trust is instead fully determined by the reputation.

The final degree of cooperation within the system may not be affected only by the selected mechanism for cooperation, but amy also be affected by physical conditions. For example, if resource is difficult to collect then cooperation may be needed in order to survive, whereas if resource is easily available agents can survive without cooperation. For this reason also the dynamics of the physical environment are described. 
The agents live in a spatial environment, which is composed of a square grid of patches. Resource grows on these patches following a logistic growth equation, and diffuses between patches. Agents can move between and harvest from patches. All agent decisions are stochastic. The probabilities that govern decision-making are determined by the state of the agent (e.g., whether it is 'hungry'), and the state of the surroundings (e.g., availability of resource, presence of other agents). All agent actions cost energy, which is replenished by harvesting resource. When an agent chooses to harvest, it may do so individually or in cooperation with a neighbouring agent. An individual harvester takes resource from its own patch only. Cooperating harvesters take resource from the two involved patches, and share this resource equally. Energy costs for harvesting are proportional to the amount of resource harvested, but the cost is higher for individual harvesters than for cooperating harvesters. Defecting agents do not invest in harvesting their own patch and thus does not take any resource from its patch; instead, they claim half of the total harvest. Defection gives a high net yield for a defector, at the cost of the yield of its interaction partner.

In terms of studying resilience it is relevant to also consider the possibilities of agents changing their behaviour, which may result in changes in the 'social' resilience of SES. In the model it is assumed that agents can change their behaviour in two ways, namely due to learning and due to adaptation. Learning typically takes place on shorter time-scales, and is based on the memories that individual agents have of previous interactions. Agents may learn to cooperate with agents that cooperated in previous interactions. Adaptation occurs through natural selection that operates on the agent population level, and typically works through a hereditary mechanism that take place on longer time-scales. This mechanism was considered earlier by Ten Broeke et al. (2017). The values of hereditary parameters remain constant over the lifetime of an individual agent, although there is diversity in the values they have for different agents. When new agents are born, they 'inherit' the parameter values of their parents with some random deviation. As not all agents are equally successful, bias in parameter distributions may appear over time, i.e. evolution occurs. Hereditary parameters in the model are $w_{\text {harvest }}^{a}$ and $w_{\text {move }}^{a}$, which affect the probabilities to harvest or move of agent $a$. The parameter $w_{r e p}^{a}$ represents the relative weight of reputation versus memory in computing trust values. The parameter $w_{\text {defect }}^{a}$ can make an agent more likely to defect rather than cooperate, or vice versa. Finally, the parameter $w_{\text {reciprocity }}^{a}$ determines whether the agent is likely to reciprocate previous behaviour of its interaction partner when deciding on whether to defect or cooperate.

\subsection{Model analysis methodology}

\subsubsection{Output measures}

In ABMs data is generated not only on the system-level, but also on the level of agents and patches. A single model run generates very large amounts of data. To gain a good understanding of model behaviour, proper output measures need to be selected. Basic system-level output measures are the total population size $n(t)$, and the total amount of resource $\sum R_{i, j}$. These measures reveal how efficient the agent population is at gathering resource, and to what extent the resource is (over-)exploited. Since the model is composed of locally interacting agents, additional measures, both on the 
agent-level and on the system-level, are needed to understand the model behaviour. In the following subsections we discuss other output measures that will be used as part of the analysis.

\section{Level of cooperation}

Two measures will be used to assess the level of cooperation in the system. Firstly, we consider the proportion of interactions that lead to cooperation

$$
n_{\text {coop }}(t) / n_{\text {int }}(t) \text {. }
$$

This measure may give a good indication of the level of cooperation in the system, but normalising based on the total number of interactions can give misleading outcomes especially if this number is very small. For example, a very small number of cooperations could look like a high level of cooperation if the total number of interactions is comparatively small. We therefore use a second measure

$$
n_{\text {coop }}(t) / n(t),
$$

with $n(t)$ the total population size. Normalising with respect to $n(t)$ prevents misleading outcomes if the number of interactions is small, but gives no information on the number of defections relative to the number of cooperative interactions. We will therefore use both measures complementary.

To gain insight into how agent behaviour develops we record all hereditary parameters, namely $w_{\text {harvest }}^{a}, w_{\text {move }}^{a}, w_{\text {rep }}^{a}, w_{\text {defect }}^{a}$, and $w_{\text {reciprocity. Histograms of }}^{a}$ these parameters will be recorded for each time-step.

\section{Clustering measure}

All interactions between agents are based on spatial proximity. As part of model analysis we therefore want to measure not only the size of the agent population, but also its spatial distribution. To this end we will use the following measure for spatial clustering of agents,

$$
\gamma(t)=\frac{L^{2}}{n(t)-1} \sum_{a=1}^{n(t)} \frac{n_{<n n>}^{a}(t)}{5}
$$

with $n(t)$ the total number of agents at time $t$, and $L^{2}$ the total number of sites on the square field. The sum runs over all agents and $n_{<n n>}^{a}(t)$ denotes the number of agents on the same patch as agent $a$, or one of the four neighbouring patches. Note that Table 6.1 lists all the used symbols in the main text. Eq. 6.3 measures the mean density of agents on the same or neighbouring patches as other agents, divided by the mean density of agents across all patches. The mean density of agents on the same and neighbouring patches is given by the sum in Eq. 6.3. The mean density of agents across all patches is calculated as $(n(t)-1) L^{-2}$. A value of $\gamma(t)=1$ means that both densities are equal, i.e. there is no spatial clustering. A value larger than 1 
means that agents tend to be located at patches with more neighbouring agents than average. A value smaller than 1 means that agents tend to be located at patches with fewer neighbouring agents than average.

\subsubsection{Resilience}

The main aim of this paper is to analyse the effect of cooperation on resilience. It is not straightforward to specify resilience, since many definitions are in use (Grimm and Wissel 1997, Brand and Jax 2007, Folke et al. 2010). We distinguish here between resilience to changes in the model hereditary parameters and resilience to shocks, i.e. resilience in terms of long-term and short-term time-scales respectively.

\section{Resilience to parameter changes}

The resilience of the population also depends on biophysical parameters. In particular, the carrying capacity $K$ represents the maximal amount of resource that can be present on a site. The analysis of an earlier version of the basic model version without cooperation shows that a number of tipping points exist where the population collapses (Ten Broeke, Van Voorn and Ligtenberg 2016), i.e. if the value of $K$ is decreased, the available resource may become insufficient to support a stable population of agents. The presence of a hereditary mechanism that allows for adaptation improves the resilience against such tipping points (Ten Broeke et al. 2017). Here we will analyse whether these tipping points are affected by the presence of cooperation. For quantification, we will measure the location of tipping points in parameter space and then verify whether this location is affected by the presence of cooperation by comparing the base model to model versions with mechanisms for cooperation.

\section{Resilience to shocks}

Resilience is not only relevant regarding long-term time-scales, but also in terms of shocks with a short-term time-scale, as adaptation through hereditary mechanisms is not fast enough to operate on shocks. The measure for resilience to shocks is based on return time (Gunderson 2000). First, the model is run for a large number of timesteps until the output has stabilised. Note that, due to stochasticity, the model output will always show fluctuations after this stabilisation. Once the model has stabilised a shock is introduced. The strength of the shock is measured as the sudden decrease in resource $R_{i, j}(t)$ on all sites $i, j$. Alternatively, a shock can be a temporary change in $r$ (the resource growth rate) or $R_{\max }$ (the maximum harvest size), the latter which represents a higher difficulty for agents to gather resource. The effects on the various output measures are recorded, specifically

- the decrease in population size caused by the shock (this can be complete extinction of the population), and

- the time required for the population to recover from the shock.

The decrease in population size caused by the shock (i.e., the effect of the shock) is quantified by measuring the difference between population size at the time of the shock and its minimum value before the population starts to recover 


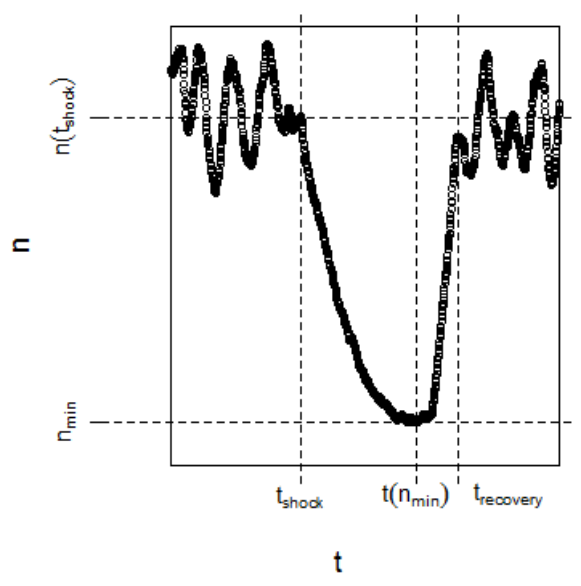

Figure 6.2: A typical response of the model to a shock. Here $t_{\text {shock }}$ denotes the time of the shock, $n_{\min }$ the minimum population reached after the shock, and $t_{\text {shock }}$ the time at which the population has recovered.

$$
\Delta n_{\text {shock }}=n\left(t_{\text {shock }}\right)-n_{\text {min }}
$$

with $t_{\text {shock }}$ the time at which the shock is applied, and $n_{\min }$ the minimum value of $n$ that is reached following the shock. Fig. 6.2 shows $t_{\text {shock }}, n_{\text {min }}$, and other relevant measures for a typical response to a shock in the model. If the resource level is low after the shock, a number of time-steps may pass before the resource level is high enough for the population size to start increasing. To determine the time when the population starts to increase, consecutive values of $n$ will be compared, each averaged over a short time-window. This averaging is done to remove short-term random fluctuations of $n$. Whether the shock causes extinction is measured by verifying whether $n$ reaches zero before the population starts to recover. The recovery time is measured as the time between the start of the recovery, and the moment where the population stops to increase

$$
\Delta t_{\text {recovery }}=t_{\text {recovery }}-t_{n_{\text {min }}} .
$$

Again, $t_{\text {recovery }}$ is determined by comparing consecutive time-windows.

\subsubsection{Sensitivity analysis}

To analyse the model, we use a variety of sensitivity analysis methodologies. Sensitivity analysis quantifies the effects of parameter variations on model output (Cariboni et al. 2007, Ten Broeke, Van Voorn and Ligtenberg 2016). In this paper both global and one-factor-at-a-time sensitivity analysis will be used. 


\section{Global sensitivity analysis}

In global sensitivity analysis all parameters are varied simultaneously across a region of parameter space (Cariboni et al. 2007). The variation of the model output across this range is quantified by calculating the variance and decomposed the variance into terms that are attributed to the various model parameters (i.e., conditional variances). The model parameters can then be ranked based on how strongly they influence the model output. Since parameters are varied simultaneously interaction effects may be taken into account. However, aggregating the model output across a region of parameter space makes it difficult to infer causal relationships between specific parameters and model outputs (Ten Broeke, Van Voorn and Ligtenberg 2016). We therefore supplement global sensitivity analysis with one-factor-at-a-time sensitivity analysis.

\section{One-factor-at-a-time sensitivity analysis (OFAT)}

OFAT examines the effect of an individual parameter on the model output while keeping the other parameter values constant. Model outputs are plotted as a function of the varied parameter. The main advantage of OFAT is that it shows clearly the relationship between individual parameters and the model output, including whether this relationship is linear or non-linear. Furthermore, OFAT is a better tool for detecting tipping points than global sensitivity measures (Ten Broeke, Van Voorn and Ligtenberg 2016, Ten Broeke et al. 2017).

Table 6.1: List of used symbols.

\begin{tabular}{|l|l|l|}
\hline Symbol & Description & Units/range \\
\hline$a$ & Index for agents & $0,1,2, \ldots$ \\
$i, j$ & Indices for patches & {$[1, L]$} \\
$K$ & Carrying capacity & mass \\
$L$ & Lattice size & distance \\
$n(t)$ & Number of agents & $0,1,2, \ldots$ \\
$n_{<n n>}^{a}(t)$ & Number of nearest neighbours for agent $a$ & $0,1,2, \ldots$ \\
$n_{\text {coop }}(t)$ & Number of cooperations & $0,1,2, \ldots$ \\
$n_{\text {int }}(t)$ & Number of interactions & $0,1,2, \ldots$ \\
$r$ & Resource growth rate & time \\
$R_{\text {ij }}(t)$ & Resource at patch $i, j$ at time $t$ & mass \\
$R_{\text {max }}$ & Maximum harvest size & mass \\
$R_{\text {shock }}$ & Resource level after shock & mass \\
$t$ & Time & time \\
$w_{\text {defect }}^{a}$ & Parameter representing probability to defect & {$[0,1]$} \\
$w_{\text {harvest }}^{a}$ & Harvest coefficient & {$[0, \rightarrow\rangle$} \\
$w_{\text {move }}^{a}$ & Move coefficient & {$[0, \rightarrow\rangle$} \\
$w_{\text {reciprocity }}^{a}$ & Weight assigned to reciprocity in interactions & {$[0,1]$} \\
$w_{\text {rep }}^{a}$ & Weight assigned to reputation in interactions & {$[0,1]$} \\
$z$ & Variation in offspring traits & {$[0,1]$} \\
$\gamma(t)$ & Clustering measure & {$[0, \rightarrow\rangle$} \\
\hline
\end{tabular}




\subsection{Results}

\subsubsection{Default parameter setting}

For benchmarking the different model versions are first studied at default parameter settings. In the full model version, which contains all mechanisms for cooperation, a stable high level of cooperation is maintained (Fig. 6.3), while the trajectories of the agent parameters are also stable, as is shown for $w_{\text {defect }}$ in Fig. 6.3.

In Fig. 6.4a it can be seen that any mechanism promoting cooperation leads to more or less the same (high) level of $n(t)$, whereas absence of such a mechanism gives rise to very low values of $n(t)$. Lack of a mechanism to support cooperation makes the population as a whole less efficient in using the available resources. The decreased population size is accompanied by a decrease in the level of cooperation between agents (Fig. 6.4b). In the base model (black), around $40 \%$ of interactions end in cooperation, and the remaining interactions lead to defection. In the reciprocity model (blue) and the partner choice model (green) the level of cooperation is clearly higher than in the base model. In the voluntary interaction model (red), the level of cooperation is lower, but the total number of interactions is also much lower (Fig. 6.4c). Thus, even though most interactions end in defection, the number of defections is lower than in the base model. These results suggest that cooperation is better promoted by mechanisms other than a mechanism for voluntary cooperation.

Even though mechanisms for reciprocity positively affect the level of cooperation, the finding that cooperation in the base model is common even without reciprocity may seem surprising. In absence of any mechanism to support cooperation, it might be expected that agents would never cooperate. Yet the results in Fig. 6.4b,c show that cooperation in the base model is common. To understand this finding, note that the base model shows a strong degree of spatial clustering of agents. For example, Fig. 6.5 shows a typical spatial arrangement of agents in the base model. Fig. 6.6 shows the amount of clustering as function of time for each of the model versions. Clearly spatial clustering is very strong in the base model, whereas the other model versions show little clustering on long time-scales. The presence of these clusters leads to an increase in the level of cooperation. Clusters with high defection rates disappear quickly from the simulation, because defections hinder the survival of interaction partners. Clusters with a high level of cooperation, in contrast, can survive for extended periods. However, such clusters are susceptible to exploitation by agents that defect often. This susceptibility limits the growth of these clusters. As a result, some regions of the lattice remain completely empty, which explains the large difference in population size between the model versions (Fig. 6.4a).

\subsubsection{Sensitivity analysis}

The qualitative effects of cooperation on model output are evaluated using the OFAT analysis for all of the model parameters. Given the large number of parameters, we limit the discussion to only a few parameters that affect the model behaviour strongly. To further quantify the effects of cooperation sensitivity analysis is performed for both the base and the full model. Details of the global sensitivity analysis are given in 6.C. The main outcome of the global sensitivity analysis is that both model versions contain tipping points where the population goes extinct. Out of 1000 sample points, 


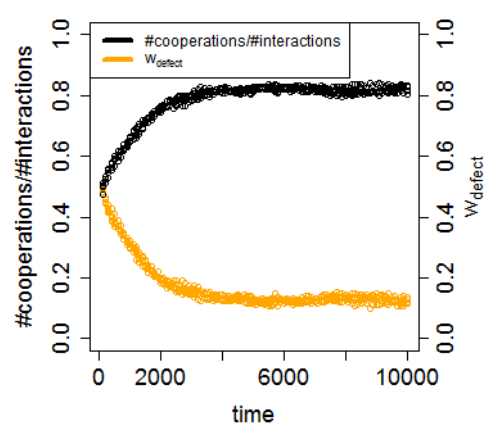

Figure 6.3: Proportion of interactions that end in cooperation, as function of time, in the full model (black). Mean value of $w_{\text {defect }}$ as function of time, in the full model (orange).

in the basic model 790 runs converged to extinction, while in the model version with cooperation 722 runs converged to extinction. In the following paragraphs we will discuss the transition towards extinction in more detail.

\section{Maximum harvest size}

In the selection of the default parameter setting, we have assumed conditions for the interactions between agents and the environment. Given that a decrease in the level of cooperation leads to a strong decrease in population size, the conditions have been chosen such that it is known to be difficult to survive without cooperation (Ten Broeke et al. 2017). We change the value of the maximum harvest size $R_{\max }$ to modify these assumptions. Low values of $R_{\max }$ mean that resource can only be gathered slowly, whereas high values mean that it can be gathered more quickly. Fig. 6.7a shows that increasing $R_{\text {max }}$ initially leads to an increase in $n$ for the base model. This increase is followed by a decrease when $R_{\max }$ is further increased. The full model, shows a similar pattern, but the peak in $n$ is located at a lower value of $R_{\max }$. The decrease in population size is caused by over-exploitation of the resource. Due to the higher harvest size, agents are collectively able to gather large amounts of resource, which causes resource scarcity. In turn, this scarcity leads to a decline in population size. Fig. $6.7 \mathrm{c}$ shows that scarcity increases as $R_{\max }$ increases. For small values of $R_{\max }$, scarcity is higher in the full model, but for larger values the two models are similar.

As $R_{\max }$ is increased, the level of cooperation in the full model decreases. In the base model the level of cooperation also shows a strong decrease. Since resource can be harvested more quickly, groups of agents can be sustained with lower levels of cooperation. Thus, the pressure on individual agents to maximise resource gains through defection becomes stronger than the pressure on groups of agents to cooperate. In contrast, further increasing $R_{\max }$ leads to a gradual increase in the level of cooperation in the base model. This increase, however, is caused by the presence of increasing fluctuations in the population size $n(t)$ and the resource level $R_{i j}(t)$. These fluctuations lead to variations in the value of $w_{\text {defect }}$. Since $w_{\text {defect }}$ is close to 


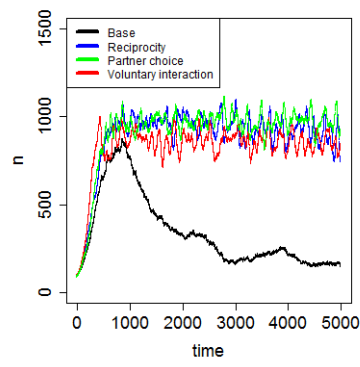

(a)

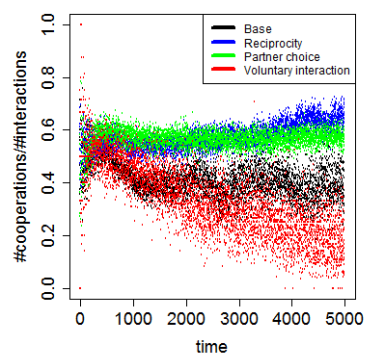

(b)

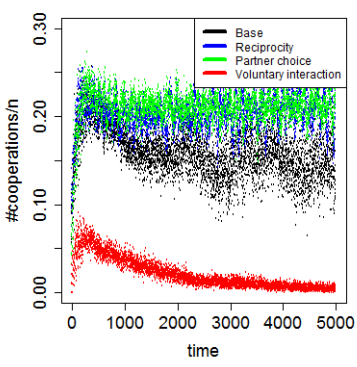

(c)

Figure 6.4: (a): Number of agents $n$ as function of time with all parameters at the default values. The colours correspond to different mechanisms to support cooperation. (b): Proportion of interactions that end in cooperation, as function of time. (c): Number of interactions that end in cooperation as function of time, relative to the population size $n$.

its maximum value of 1 for $R_{\max }=0.3$, these variations lead to an average decrease in $w_{\text {defect }}$ and an increase in the level of cooperation.

\section{Resource availability}

The previous section has shown that resource availability and scarcity may be important factors in determining the effects of cooperation on the system. Here we explore the effects of varying levels of resource availability more directly by varying the carrying capacity $K$. Increasing $K$ leads to an increase in the resource availability, since each site can contain a larger amount of resource. In Appendix 6.B we show that increasing resource availability through changing the resource growth rate $r$ yields similar results to increasing $K$. Increased resource availability is expected to lead to an increase in population size $n$. Fig. 6.8a shows that for $K \leq 0.5$ the resource availability is insufficient to maintain a population. In the full model $n$ increases as $K$ is increased, as expected. Individual time series show that for increasing values of $K$ the population size $n$ shows cyclic behaviour. This result is known in ecology as the paradox of enrichment (Rosenzweig 1971). The basic model also shows that $n$ increases with $K$, but only for small values of $K$. For larger values of $K$ the population starts to decrease. This decrease is caused by a decrease in the level of cooperation $6.8 \mathrm{~b}$. The basic model shows no cyclic behaviour.

In Fig. 6.9 we show the distribution of $w_{\text {defect }}^{a}$ across agents, for different values of $K$. Large values of $w_{\text {defect }}^{a}$ indicate that agents defect often, while low values indicate that they cooperate often. The distribution clearly shows that agents in the base model defect more often than in the full model. Furthermore, in the full model $w_{\text {defect }}$ is on average smaller for high values of $K$. This indicates that agents cooperate even more often for large values of $K$. In the base model, $w_{\text {defect }}$ is on average higher for large values of $K$. Thus, agents in the base model cooperate less if resource availability is high. Thus, whereas in the full model more favourable environmental conditions support a larger population, in the base model such conditions lead to increased competition between agents, which in turn causes a decrease in the population size. 


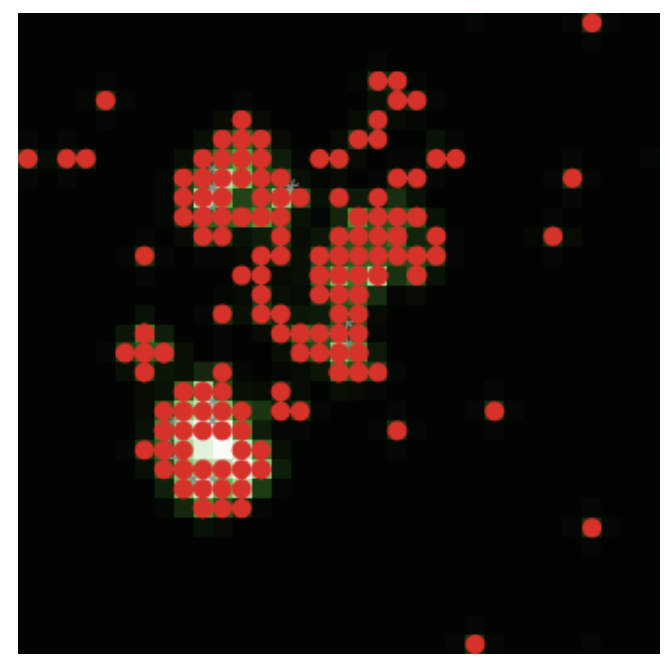

Figure 6.5: Snapshot of a typical spatial configuration of agents in the base model, with all parameters at their default values. The background colour of sites shows the resource density, with dark sites having large densities, and light sites having low densities. The red circles correspond to agents. Note that most agents are spatially clustered into groups.

\subsubsection{Resilience to shocks}

For quantification of the resilience of the model to shocks the model is first run for 10.000 time-steps without manipulations before applying the shock. Each measurement is performed both for the base model and the full model, and for each version we perform 10 replicates. We consider three types of shocks, namely shocks to the resource level $R_{i j}(t)$, to the resource growth rate $r$, and to the maximum harvest size $R_{\max }$. Shocks to $R_{i j}(t)$ are instantaneous. Shocks to $r$ and $R_{\max }$ are applied for a duration of time. This duration is varied between 50 and 500 time-steps.

\section{Resource level}

Firstly, we consider shocks in the resource level $R_{i j}(t)$ by setting the resource level $R_{i j}(t)$ of each site equal to a pre-defined value $R_{\text {shock}}$, at $t=10.000$. The value of $R_{\text {shock }}$ is varied between $5 \%$ of carrying capacity and $0.5 \%$ of carrying capacity. On sites for which $R_{i j}(10.000)<R_{\text {shock }}$ the resource level remains unchanged. A low value of $R_{\text {shock }}$ thus corresponds to a strong shock. Strong decreases in the resource level, as modelled here, can occur in real-life cases, for instance in the case when natural disasters occur.

The results show that both model versions are strongly resilient to shocks in $R_{i j}(t)$. In the full model version the shock is followed by a strong decrease in $n(t)$, but recovery follows quickly (Fig. 6.10). Even when the resource level is set close to zero, a positive population is maintained. In the base model the population size before the shock is smaller than in the full model. The shock is followed by a further decrease in population size, but a positive population is maintained. The recovery 


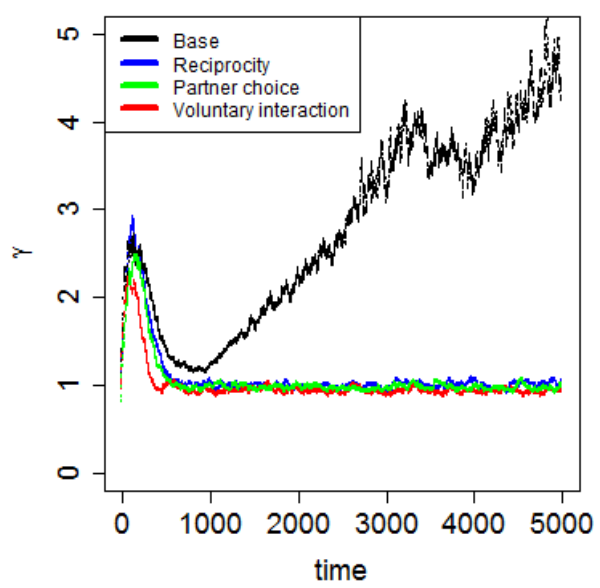

Figure 6.6: Clustering measure $\gamma(t)$ as function of time. The base model shows an increasing amount of clustering.

time is longer in the full model than in the base model, but this is likely because the full model returns to a larger population size than the base model. Thus, regardless of the level of cooperation, the model is resilient to instantaneous shocks in the resource level. This resilience may be due to the internal energy that agents have stored at the time of the shock. Following the shock, this energy allows agents to survive for some time while the resource replenishes.

\section{Resource growth rate}

To quantify the effects of shocks with an extended duration we lower the resource growth rate to $r=0.01$ for various durations. In real-life cases such a decreased growth rate may occur as a result of degradation of the environment. For example, in grasslands drought or overgrazing can cause a transition into such a degraded state (Crépin and Lindahl 2009). In the model, the decreased growth rate is insufficient to support a stable population on long time-scales. The results show that for the full model the population size after the shock decreases as the shock duration increases (Fig. 6.11). In contrast, in the base model the population size is not strongly affected by the duration of the shock. The difference is likely caused by the difference in $n(t)$ before the shock. In absence of shocks the full model has a larger population size, and lower resource levels. For strong shocks, both populations decrease to approximately the same level. However, compared to the population size before the shock, the decrease in population is small for the base model, and much larger for the full model. Thus, whereas the base model is less affected by the shocks than the full model, there is little difference between the resulting population sizes following the shock. Since the full model is closer to the point of over-exploitation, shocks to the growth rate have a relatively strong effect. 


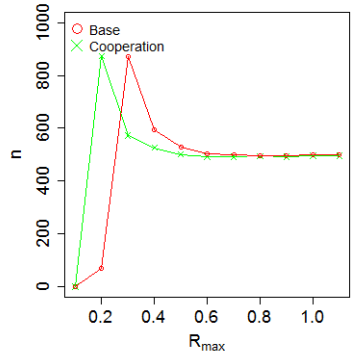

(a)

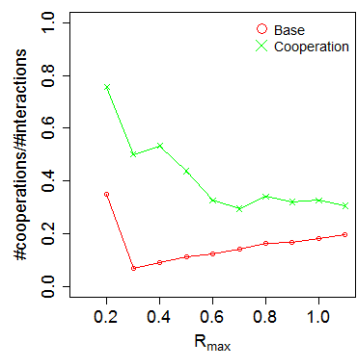

(b)

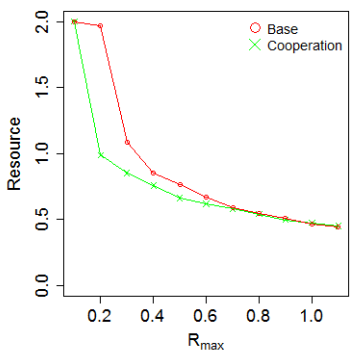

(c)

Figure 6.7: (a): Number of agents $n$ as function the parameter $R_{\max }$. (b): Proportion of interactions that lead to cooperation, as function of the parameter $R_{\max }$. (c): Average amount of resource per patch, as function of $R_{\max }$. In all three graphs the output was averaged over time between $t=10.000$ and $t=20.000$.

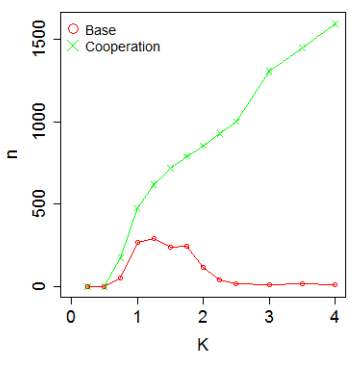

(a)

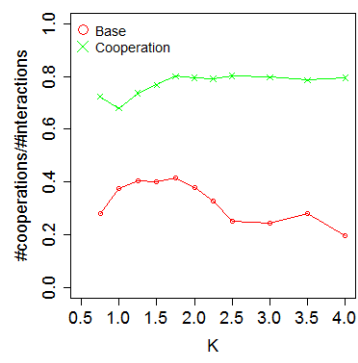

(b)

Figure 6.8: (a): Number of agents $n(t)$ as function the parameter $K$. (b): Proportion of interactions that lead to cooperation, as function of the parameter $K$. In both graphs the output was averaged over time between $t=10000$ and $t=20000$. 


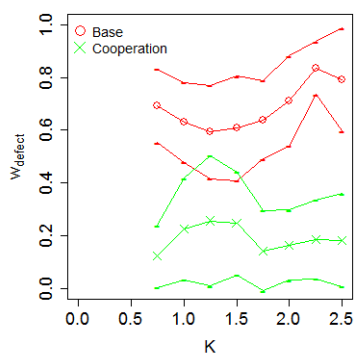

(a)

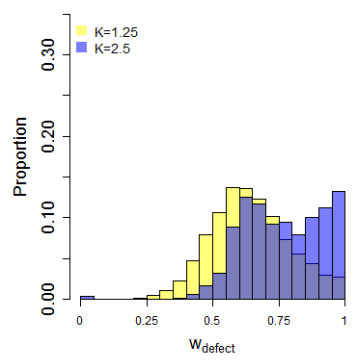

(b)

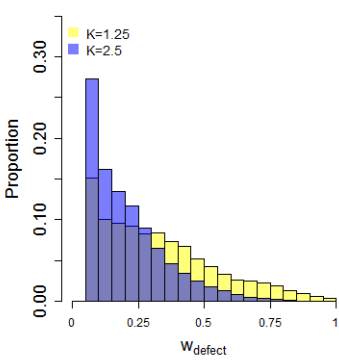

(c)

Figure 6.9: (a): Mean value of $w_{\text {defect }}^{a}$ as function of $K$, for the basic model and the model with cooperation, averaged over time between $t=10000$ and $t=20000$. The horizontal lines show the mean plus or minus one standard deviation. (b): Histogram of $w_{\text {defect }}^{a}$ for the basic model, for $K=0.75$ and $K=2.5$, averaged over time between $t=10000$ and $t=20000$. (c): Histogram of $w_{\text {defect }}^{a}$ for the full model, for $K=0.75$ and $K=2.5$, averaged over time between $t=10000$ and $t=20000$.

\section{Maximum harvest size}

The comparison between the base model and the full model in the above paragraphs is somewhat obscured by the difference in population size between both versions. Since the population size in the base model is lower than in the full model, the population requires less resource. Due to this difference in population size, shocks to the resource level or the resource growth rate may have a stronger effect in the full model. Lowering the maximum harvest size $R_{\max }$ does not directly affect resource levels, but does make it more difficult for agents to gather resource. In real-life cases such a decrease might correspond to a situation where the resource has become more difficult to harvest because more remote areas need to be accessed. Alternatively, it might refer to an agreement to limit the amounts of harvested resource.

The maximum harvest size in simulations is lowered to $R_{\max }=0.15$, while the duration of the shock is varied. In the base model the shock causes a strong decrease in $n(t)$ (Fig. 6.12). As the duration of the shock is increased, $n(t)$ decreases further. If the duration is 300 time-steps or longer, the population goes extinct. In the full model, there is a decrease in $n(t)$ following the shock, but the size of this decrease hardly depends on the duration of the shock. Due to evolution of the population recovery starts even before the end of the shock. Fig. 6.12b shows an example run with a shock duration of 500 time-steps. In response to the shock, agents adapt to cooperate more frequently. This increase in cooperation leads to an increase in $n(t)$. Thus cooperation promotes the resilience of this system against shocks to the maximum harvest size. 


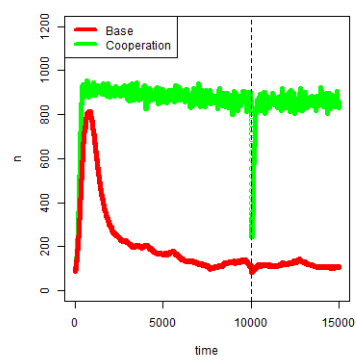

(a)

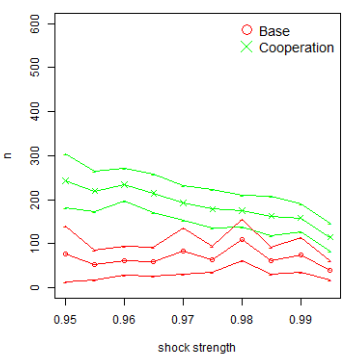

(b)

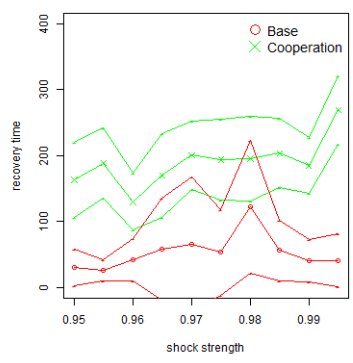

(c)

Figure 6.10: (a): Time series of the number of agents. The dashed line indicates a shock, where the resource on each site is lowered to $R_{i j}(t)=0.005$. (b): Number of agents $n(t)$ following a shock to the resource level $R_{i j}(t)$, as function of the strength of the shock. The strength on the horizontal axis is defined as $1-R_{\text {shock }} / K$, and the vertical axis was measured as the minimum value of $n(t)$ that is reached before recovery starts. (c): Time until recovery from the shock. This was measured as the number of time-steps until the output stops to increase after recovery from the shock has started. In each run, the population size returned to the original level.

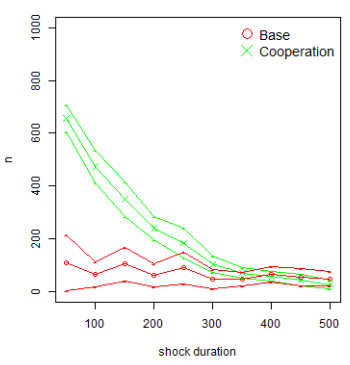

(a)

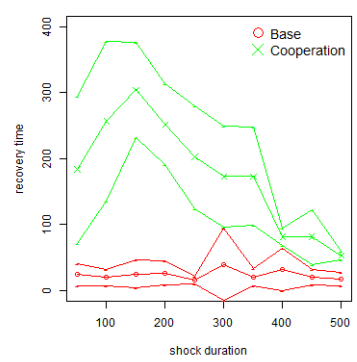

(b)

Figure 6.11: (a): Number of agents $n(t)$ following a shock to the resource growth rate $r$, as function of the duration of the shock. This number was measured as the minimum value of $n(t)$ that is reached before recovery starts. (b): Time until recovery from the shock. This was measured as the number of time-steps until the output stops to increase after recovery from the shock has started. In each run, the population size returned to the level from before the shock following this recovery. 


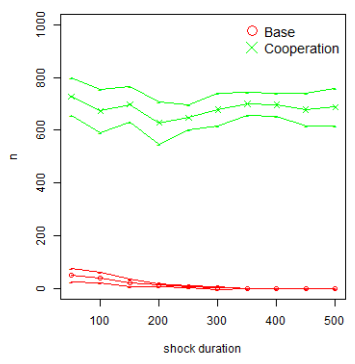

(a)

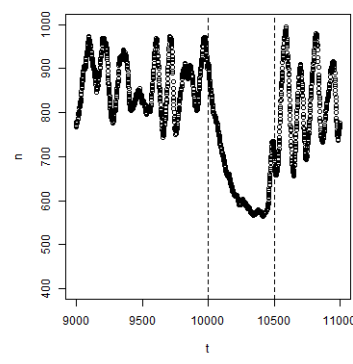

(b)

Figure 6.12: (a): Number of agents $n(t)$ following a shock to the maximum harvest size $R_{\max }$, as function of the duration of the shock. This number was measured as the minimum value of $n(t)$ that is reached before recovery starts. (b): Time series of one of the replicate runs in the full model. The start and end of the shock are indicated by the dashed lines. Note that $n(t)$ decreases following the shock, but recovery starts before the end of the shock.

\subsection{Conclusions \& Discussion}

To understand the resilience of common-pool resource systems, we need to consider the presence of cooperation in these systems. Existing game theory models have shown indirect reciprocity to be a powerful mechanism for maintaining a high level of cooperation (e.g. (Nowak and Sigmund 2005, Nowak 2006, Gotts et al. 2003, Bear and Rand 2016)). Most of these models, however, do not consider dynamic interactions between agents and their environment. In this study we have explicitly included such interactions by modelling them as part of an ABM. The results show that these interactions strongly influence the level of cooperation. Even in absence of reciprocity between agents, feedbacks from the environment are sufficient to maintain a positive level of cooperation. Including reciprocity further increases the level of cooperation.

The results show that feedbacks from the environment influence cooperation in multiple ways. When resources are difficult to collect, cooperation is needed to survive. Even without indirect reciprocity, cooperation is common under such circumstances. Although on the individual level agents might be expected to maximise their own gains by defecting from cooperation, the model shows the emergence of spatial clusters that maintain a relatively high level of cooperation. The stability of these clusters is increased by the limitation that agents cannot harvest and move in the same time-step. This limitation implies that cooperation prevents dispersal, and a group of cooperating agents will thus stay together. Furthermore, offspring are created next to the parent agent. This further limits the dispersal of agents. The effects of limited dispersal on the evolution of cooperation has previously received much attention (Kümmerli et al. 2009, West et al. 2007). Limited dispersal tends to keep relatives close. This could lead to higher levels of cooperation due to kin selection, since cooperation would tend to be directed towards relatives. Alternatively, when local resource availability is low, limited dispersal might lead to kin competition, thus lowering the level of cooperation. Experimental evidence for both of these effects has been found in experiments on bacteria (Griffin et al. 2004). While kin selection is not 
included in our model, it might be an interesting area for future research.

The trajectories of the agent parameters in the model appear to be stable, as is shown for $w_{\text {defect }}$ in the default setting in Fig. 6.3. Each of the parameters gradually converges, after which it shows only small random fluctuations around a constant mean. In the full model, this convergence leads to a stable and high level of cooperation. This contrasts with results from previous studies (Nowak and Sigmund 1998, Lindgren 1992). In Nowak and Sigmund (1998) it was found that periods with many agents that tend to cooperate were interrupted by periods with many defectors. The relative stability of the agent parameters in our study may be due to adaptation through evolution occurring relatively slowly. This adaptation occurs slowly because agents on average participate in a number of interactions before creating offspring, and the differences between offspring and parents are small. Thus, for defection to spread, on average defectors need to be successful over a large number of interactions. In contrast, in Nowak and Sigmund (1998) a much lower number of interactions per generation was used. In our model, learning occurs on similarly short time-scales. In the model, if learning is more important than cooperation, the fluctuations in the level of cooperation are larger (results not shown). This difference in time-scales over which learning and adaptation occur was not present in previous models of cooperation, and may be an interesting topic for further study.

Although our results show that reciprocity is not needed to maintain a positive of cooperation, reciprocity does further increase the level of cooperation, and increases the range of environmental conditions for which cooperation can evolve. The increased level of cooperation leads to an increased population size. The increased level of cooperation may affect the resilience of the system in different ways, depending on the type of shock. Thus, to assess the resilience it is crucial to specify both the type of shock that is applied, and the component of the system that needs to be resilient, i.e. 'resilience of what to what' (Carpenter et al. 2001). Systems with a higher level of cooperation use resources more efficiently, but also may be closer to the point of over-exploitation. As a result, shocks to the resource level can have a strong impact when they lead to sudden over-exploitation. Shocks to the maximum harvest size have little effect, because due to cooperation the agents still manage to gain sufficient energy from the harvests. In systems with little cooperation, in contrast, competition between agents makes it more difficult to obtain sufficient energy from harvests. As a result, the population size is decreased, and the system is far from over-exploitation. Therefore, shocks to the resource level hardly have any effect, whereas shocks to the maximum harvest size quickly lead to extinction.

Our study shows that the resilience of the model depends strongly on cooperation. But it depends on the circumstances whether this cooperation has a positive or a negative on the resilience. These results show that in real-life cases cooperation is a relevant factor to consider when assessing the resilience of SES. However, precisely how cooperation affects the resilience may depend on the specific case. More cooperation may lead to a higher resilience in some contexts, but in other contexts it might lead to over-harvesting. Thus, the case-specific context should be considered carefully when assessing the effects of cooperation on resilience in a real-life system. 


\section{Acknowledgements}

We thank Geerten Hengeveld for comments on a previous version of the manuscript. The research by GTB is financed by the IO/OP theme Complex Adaptive Systems at Wageningen University \& Research.

\section{A Model Description}

The model description follows the ODD (Overview, Design concepts, Details) protocol for describing individual- and agent-based models (Grimm et al. 2006, 2010).

\section{A.1 Purpose}

The suite of five models is used to investigate the effects of cooperation between agents that compete for a common-pool resource in a spatial environment on the resilience of this system.

\section{A.2 Entities, state variables, and scales}

The state variables are listed in Table 6.2. The landscape is represented by a square lattice of length $L$. Each site $i, j(i, j=1,2, \ldots, L)$ has a length of $\Delta x=1 \mathrm{~km}$. Since the model was not fitted to data, this choice was made only for computational convenience. Periodic boundary conditions are imposed, so that $i+L=i$ and $j+L=j$. Each site has a resource level $R_{i, j}$.

Agents move over the lattice and harvest resource. An agent is identified by the index $a=1,2, \ldots$ Each agent has an energy level $E^{a}$ and a location $x_{a}$. Agents have a number of state variables, all of which are listed in Table 6.2. A precise description of the function of each state variable is provided in section 6.A.7.

Time is modelled in discrete time steps of $\Delta t=1$ day. Like the distance scale of $\Delta x=1$, this was chosen for convenience.

\section{A.3 Process overview and scheduling}

The model flow chart for a single time-step is shown in Fig. 6.13. Each box corresponds to a submodel that is described in detail in the submodel section. The 'harvest' submodel consists of several other submodels, as show in Fig. 6.14. Each submodel is run for all of the patches or agents before the start of the next submodel, except where the loops in the flowchart indicate otherwise. The time-step starts with the updating of the sites. The 'grow' submodel grows resource on each site following a logistic growth equation. The 'diffuse' submodel diffuses the resource between sites following Fick's second law (Fick 1855). The updating of sites is followed by the agent actions. Each agent first 'observes' the resource level and the number of agents on neighbouring sites. The agents then decide whether to harvest, based on their internal energy level, the estimated amount of resource on their location, and the presence of other agents. If an agent does harvest, it will try to select a potential interaction partner from any agents in the von Neumann neighbourhood (Fig. 6.14). If there are no other agents within this neighbourhood, the harvester will immediately collect resource from its 
site. If a partner is found, then it will decide whether or not to interact with that agent, based on the trust it has towards the agent and the available resource on that agent's site. If it chooses not to interact, it will proceed to collect resource from its own site. If it chooses to interact, then the interaction partner decides whether to defect or cooperate before resource is collected. If an agent does not harvest, it may decide to move based on the number of agents and estimated resource level on neighbouring sites. Alternatively, an agent may decide to neither harvest nor move, thus conserving energy. After all the actions of harvesting and moving have been carried out, each agent pays a fixed energy maintenance. There is a possibility that an agent will then die or breed, depending on its internal energy. The model then proceeds to the next time-step.

\section{A.4 Design concepts}

\section{Basic principles}

The model describes agents that move and harvest in a spatial environment. Based on their internal state (i.e., internal energy) and the state of the environment (i.e., available resource, presence of other agents) agents may decide to move to a new location, harvest from the present location, or wait for a better opportunity. Harvested resource is converted to internal energy, which is used to pay for daily maintenance and the actions of moving and harvesting. Harvesting may be done either individually, or in cooperation with an agent on a neighbouring site. When cooperating, agents need less energy to harvest the same amount of resource. Cooperation is thus more efficient that harvesting individually, but it puts agents at risk of 'defecting' interaction partners. A defecting agent does not invest energy in the harvest, but still claims half of the resource. As a result, the net energy gain for a defecting agent is larger than for a cooperating agent. However, defection damages the reputation of an agent and the trust it receives from other agents. In the short-term, defection will help the agent to accumulate a larger amount of resource. On the long term, however, the lowered reputation and trust may make other agents less likely to enter interactions with the agent. Thus, although defection yields an advantage on the short term, on the long term such behaviour might be punished.

\section{Emergence}

We consider the total population size $n_{t o t}$ and the distribution of $w_{h}^{a}, w_{m}^{a}, w_{r e p}^{a}$, $w_{\text {defect }}^{a}, \rho^{a}$, and $\tau^{a, a^{\prime}}$ across the population as emerging variables at the macro-level. In addition, the level of cooperation in the system is also considered emergent. This level of cooperation is measured by keeping track of how often agents choose to defect, or to cooperate, and of the trust values of the agents.

\section{Adaptation}

Agents adapt their behaviour to changes in their internal state or in their environment. Agents with low levels of internal energy tend to harvest resource with a higher probability. Agents also tend to harvest if the resource level on their site is high and the number of other agents on the site is low. Agents consider the state of the sites 


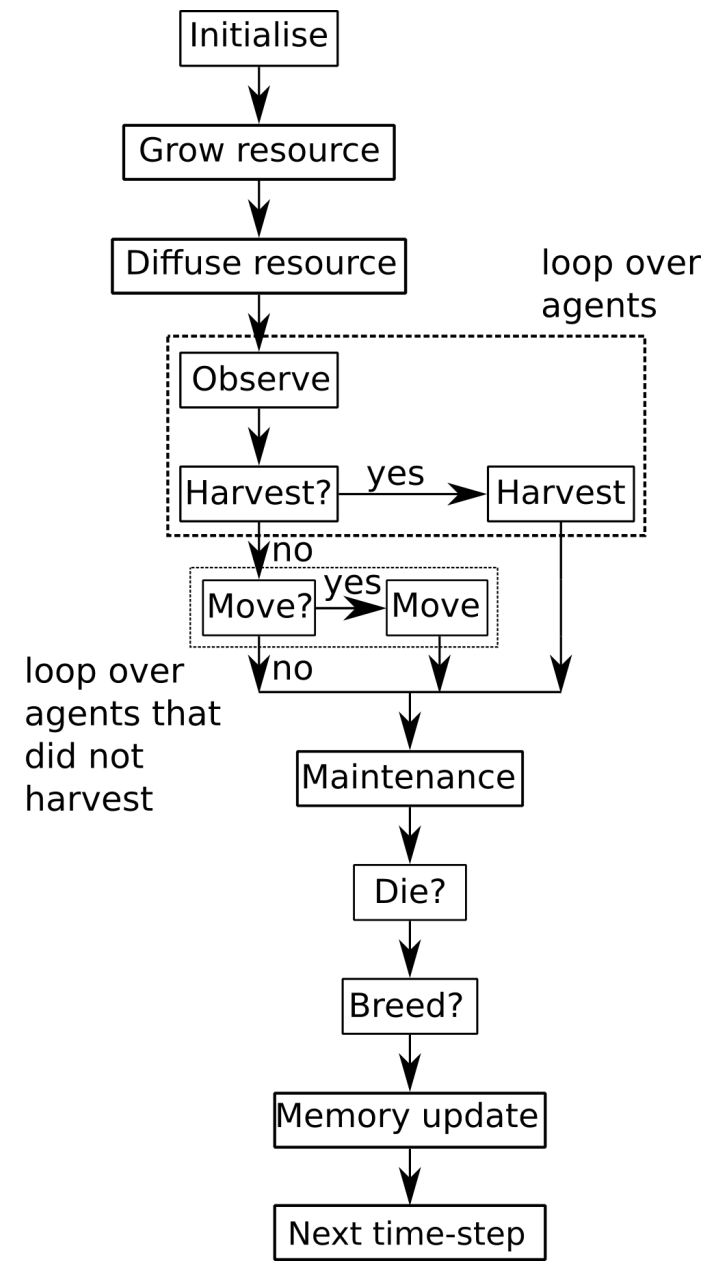

Figure 6.13: Model flowchart. The boxes correspond to the submodels explained in section 6.A.7. Each submodel is executed by all the agents or patches before the next submodel is started, except where indicated by the dashed boxes. These boxes indicate loops over agents, where each agent that enters the loop completes all the submodels within the loop before the next agent becomes active.

in the Von Neumann neighbourhood in their decisions to move to neighbouring sites. Agents are more likely to move to sites with high resource levels and few other agents. In the decision to cooperate, agents consider trust towards each other.

\section{Objectives}

The first objective of each agent is to gather sufficient resource for its own survival. The second objective is to accumulate sufficient energy to breed. 


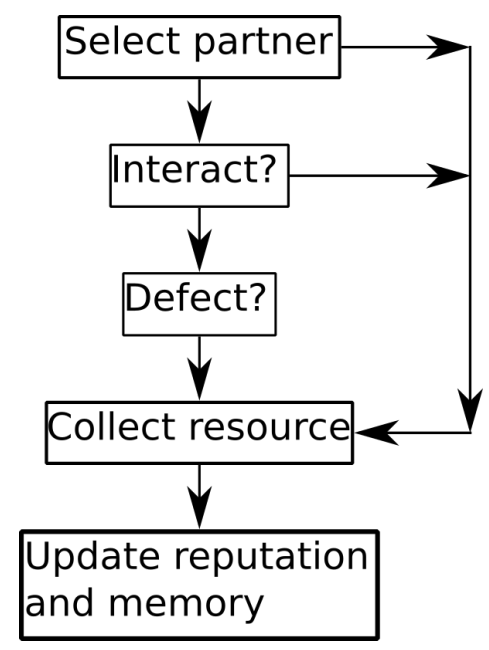

Figure 6.14: Flowchart of the harvest submodel (see Fig. 6.13). The boxes correspond to the submodels explained in section 6.A.7.

\section{Learning}

Agents learn from the outcomes of previous interactions with other agents. Agents that defect gain a lower reputation, making other agents more likely to defect in the future when the agent itself is harvesting. Furthermore, agents remember the outcomes of interactions with other agents. Unlike reputation, which is observed by all agents, these memories are only formed by the agents that were involved in the interaction. Thus, over time an agent builds a network of connections to other agents with memories of previous interactions. Through the updating of these memories and of reputation, agents learn to adjust their interactions with other agents.

Learning also occurs through the evolution of a number of adaptive agent traits. Although individual agents do not change their adaptive traits over time, the distribution of these traits over the population may change due to natural selection. When agents breed, their offspring inherit the parents' values of $w_{h}^{a}$ and $w_{m}^{a}$ with some random variation. If, for example, agents with a low value $w_{m}^{a}$ reproduce more frequently often on average, then low values will become increasingly common over time. Similarly, the agent parameters $w_{\text {rep }}^{a}, w_{\text {defect }}^{a}$, and $w_{\text {reciprocity }}$ are also inherited and may thus change due to natural selection.

\section{Prediction}

Agents tend to move less often to sites that are occupied by one or more other agents, based on the prediction that those other agents may harvest resource from the site. Agents tend to harvest more often at their present location if neighbouring locations are occupied by other agents that might move to the resource. Agents also tend to move to sites with high resource levels, predicting that the resource level will remain high and can be harvested. If an agent finds the present conditions unsuitable for harvesting, that agent may decide to wait before taking action, expecting that the 
conditions will improve in the future. Agents choose interaction partners with high trust values, based on the prediction that these agents will be less likely to defect.

\section{Sensing}

Agents sense their own internal energy. Each time step, agents assess the amount of resource on their current location and the four sites that compose the Von Neumann neighbourhood. However, agents do not sense the exact amount of resource because there is some random error. Agents also sense the presence of any other agents within the Von Neumann neighbourhood, and the reputation of those agents. There is no error in sensing which agents are present, but there is a random error in the sensing of the reputation of those agents. Agents also sense their own memory of the outcomes of previous interactions with other agents. There is no error in sensing these memories.

\section{Interaction}

Indirect interactions between agents occur through the competition for resource. In addition, agents are less likely to move to sites that contain other agents. This is a form of direct interaction. Agents are also more likely to harvest on their present site if neighbours are occupying the neighbouring sites. When an agent decides to harvest, it may ask a neighbouring agent to cooperate. Only neighbouring agents that have not yet harvested during the time-step are eligible for this interaction. The neighbouring agent then has the option of defecting, cooperating, or refusing the interaction. If the agent either defects or cooperates, it will not be able to harvest or move for the remainder of the time-step. If it refuses the interaction, it can still perform these actions.

If the interaction partner cooperates, both agents invest equally in the harvest, and equally share the harvested resource. Cooperating agents need less resource than individual harvesters to obtain the same harvest. If an agent defects, it will claim half the harvest at no investment, but this will lower its reputation, and the trust it receives from the harvester. This decrease in reputation and trust will make other agents more likely to defect during future harvests of the agent. Compared to defection, cooperation requires a larger investment, and therefore has a smaller net yield. However, cooperation increases the trust and reputation of the agent and may therefore be a more successful long-term strategy.

\section{Stochasticity}

The decisions of agents to harvest at the present location or to move to a neighbouring site are based on probability functions. The functions take as input the number of agents and the estimated amount of resource within the Von Neumann neighbourhood. This estimated amount of resource differs from the actual amount. The difference is drawn from a normal probability distribution. The decision of whether to interact is stochastic, and the probability is determined by the trust towards the interaction partner. The decision of whether to defect is also determined by a probability.

Birth and death of agents are also stochastic, but based on the internal energies of agents. If an agents breeds, the offspring inherit the values of $w_{h}^{a}, w_{m}^{a}, w_{\text {defect }}^{a}$, and $w_{r e p}^{a}$ with a small random variation. Furthermore, the agents' initial locations, 
energies, reputations, and values of $w_{h}^{a}, w_{m}^{a}, w_{\text {defect }}^{a}, w_{r e p}^{a}$, and $w_{\text {reciprocity }}$ are also stochastic.

\section{Collectives}

The model contains no predefined collectives, but collectives may emerge from the agent interactions. Agents remember what other agents they have interacted with, and develop trust based on the outcomes of those interactions. Agents are more likely to cooperate with another agent if they trust that agent. As a result collectives might emerge of agents that repeatedly cooperate out of trust.

Since interactions are based on spatial proximity, collectives may also emerge based on the spatial location of agents. Agents that are spatially close may have multiple interactions over time. Agents that often defect will have a low reputation, which may provoke further defections from surrounding agents. Thus collectives may form of agents with low reputation and little cooperation. Alternatively, agents that do not defect will get a higher reputation, creating a positive feedback towards more cooperation. Note that whereas on the short term it is beneficial for individual agents to defect, a group of agents will on average gather the most energy if all group members cooperate. Thus, it is possible in principle for such a group to outcompete groups with less cooperation.

\section{Observation}

Output variables may be recorded every time-step, but the model contains an option to record the output only at set intervals. For model runs over a large number of timesteps, the output is recorded every 100 time-steps. The collected output variables are the total number of agents $n$, the averages and standard deviations of the agent parameters $w_{h}^{a}, w_{m}^{a}, w_{r e p}^{a}, w_{d e f e c t}^{a}$ over all agents, and the averages and standard deviations of the agent state variables $\rho^{a}$ and $\tau^{a, a^{\prime}}$.

\section{A.5 Initialization}

All sites are initialized with resource levels equal to $R_{0}$ times carrying capacity. The initial number of agents is specified as a parameter, $n_{0}$. Each agent is placed on a random site with an internal energy that is drawn from a uniform distribution between 0 and the minimum energy that is needed for procreation. The initial reputation is drawn from a uniform distribution between 0 and 1 . The initial values of the agent parameters $w_{h}^{a}, w_{m}^{a}, w_{r e p}^{a}, w_{\text {defect }}^{a}$, and $w_{\text {reciprocity }}^{a}$ are drawn from normal distributions. The mean and standard deviation of these distributions are given as parameter values. The default parameter values of the model are listed in Table 6.2. Note that the tails of the normal distributions lie outside of the possible range of values for the parameters. If a value is drawn outside this range, the agent is instead given the extreme value that lies within the range. 
Table 6.2: List of used symbols in the model description. The first part contains patch state variables, the second part agent state variables, the third part parameters and initial conditions and the fourth part other symbols. Note that by state variables we refer only to basic variables that cannot be computed from other variables.

\begin{tabular}{|c|c|c|c|}
\hline Symbol & Description & Dim./range & Value \\
\hline \multicolumn{4}{|c|}{ Patch state variables } \\
\hline$i, j$ & Position indices & $0,1, \ldots, L$ & - \\
\hline$R_{i, j}$ & Resource on patch & mass & - \\
\hline \multicolumn{4}{|c|}{ Agent state variables } \\
\hline$a$ & Agent index & $0,1, \ldots$ & \\
\hline$E^{a}$ & Internal energy & energy & - \\
\hline$h^{a, a^{\prime}}$ & Time since last interaction & time & - \\
\hline$R_{i, j}^{a}$ & Resource estimate by agent & mass & - \\
\hline$w_{\text {defect }}^{a}$ & Defection coefficient & {$[0,1]$} & - \\
\hline$w_{h}^{a}$ & Harvest coefficient & {$[0, \rightarrow\rangle$} & - \\
\hline$w_{m}^{a}$ & Move coefficient & {$[0, \rightarrow\rangle$} & - \\
\hline$w_{\text {reciprocity }}$ & Weight of reciprocity for defection & {$[0,1]$} & - \\
\hline$w_{r e p}^{a}$ & Weight of memory & {$[0,1]$} & - \\
\hline$x^{a}$ & Position & patch & - \\
\hline$\rho^{a}$ & Reputation & {$[0,1]$} & - \\
\hline$\tau^{a, a^{\prime}}$ & Memory score & {$[0,1]$} & - \\
\hline \multicolumn{4}{|c|}{ Parameters and initial conditions } \\
\hline$c$ & Efficiency (yield) & energy mass $^{-1}$ & 0.9 \\
\hline$D$ & Diffusion coefficient & length $^{2}$ time $^{-1}$ & 0.1 \\
\hline$E_{b}$ & Birth energy & energy & 5 \\
\hline$E_{m}$ & Energy cost of maintenance & energy & 0.1 \\
\hline$E_{\text {move }}$ & Move cost & energy & 0.5 \\
\hline$h_{\max }$ & Maximum memory time & time & 10 \\
\hline$K$ & Carrying capacity & mass & 2 \\
\hline$L$ & Lattice size & distance & 33 \\
\hline$n_{0}$ & Initial number of agents & $0,1,2, \ldots$ & 100 \\
\hline$r$ & Growth rate & time $^{-1}$ & 0.1 \\
\hline$R_{0}$ & Initial resource & prop. of $K$ & 1 \\
\hline$R_{\max }$ & Maximum harvest & mass & 0.5 \\
\hline$R_{\text {unc }}$ & Uncertainty of resource estimates & mass & 0.1 \\
\hline$v_{b}$ & Birth coefficient & energy $^{-1}$ & 10 \\
\hline$v_{d}$ & Mortality coefficient & energy $^{-1}$ & 10 \\
\hline$w_{\text {defect }, \mu}$ & Mean of initial values of $w_{d e f e c t}^{a}$ & {$[0,1]$} & 0.5 \\
\hline$w_{\text {defect }, \sigma}$ & Sd. of initial values of $w_{\text {defect }}^{a}$ & {$[0,1 / 4]$} & 0.1 \\
\hline$w_{h, \mu}$ & Mean of initial values of $w_{h}^{a}$ & {$[0, \rightarrow\rangle$} & 0.5 \\
\hline$w_{h, \sigma}$ & Sd. of initial values of $w_{h}^{a}$ & {$[0, \rightarrow\rangle$} & 0.1 \\
\hline$w_{m, \mu}$ & Mean of initial values of $w_{m}^{a}$ & {$[0, \rightarrow>$} & 0.5 \\
\hline$w_{m, s \sigma}$ & Sd. of initial values of $w_{m}^{a}$ & {$[0, \rightarrow\rangle$} & 0.1 \\
\hline$w_{\text {reciprocity }, \mu}$ & Mean of initial values of $w_{\text {reciprocity }}^{a}$ & {$[0,1]$} & 0.5 \\
\hline$w_{\text {reciprocity }, \sigma}$ & Sd. of initial values of $w_{\text {reciprocity }}^{a}$ & {$[0,1 / 4]$} & 0.1 \\
\hline
\end{tabular}


Table 6.2 - continued from previous page

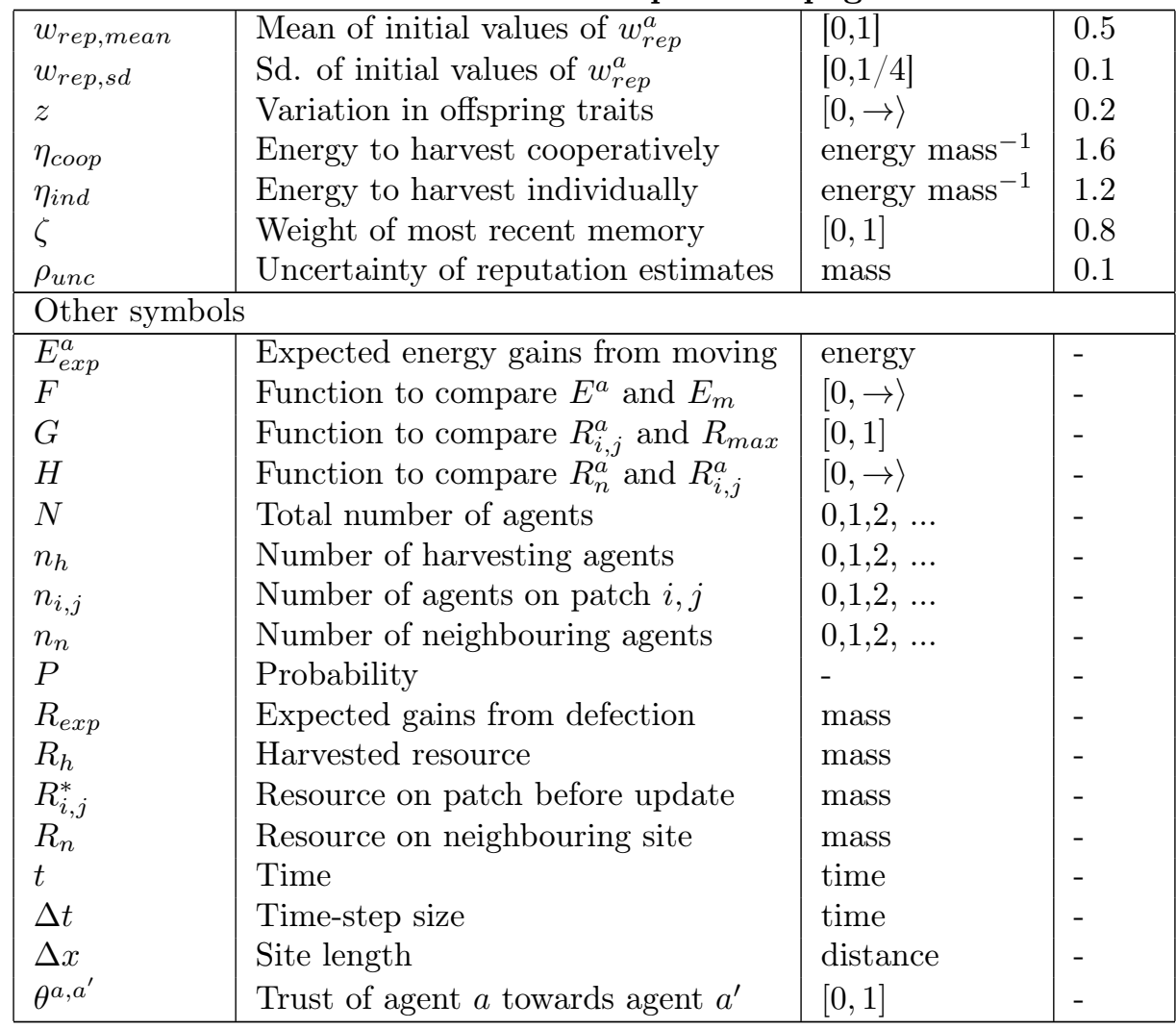

\section{A.6 Input data}

The model does not use input data to represent time-varying processes.

\section{A.7 Submodels}

\section{Grow resource}

Resource grows on sites according to a logistic growth equation

$$
\frac{d R_{i, j}}{d t}=K r\left(1-\frac{R_{i, j}}{K}\right)
$$

Each time-step the sites are updated using the analytical solution of this equation

$$
R_{i, j}=\frac{R_{i, j}^{*} K e^{r \Delta t}}{K+R_{i, j}^{*} e^{r \Delta t}}
$$


Note that several processes can change the resource on a site during the same timestep. In order to keep the notation simple, we use $R_{i, j}^{*}$ to denote the resource before the update in each of these processes, instead of having different symbols for the resource before and after each of the separate processes.

\section{Diffuse resource}

The resource diffuses following Fick's second law (Fick 1855)

$$
\frac{d R_{i, j}}{d t}=D \nabla^{2} R_{i, j}
$$

with $\nabla=\left(\frac{\partial}{\partial x}, \frac{\partial}{\partial y}\right)$. The equation is discretised in space using the central difference and discretised in time using a forward Euler algorithm. This yields

$$
R_{i, j}=\left(1-\frac{4 \Delta t}{\Delta x^{2}} D\right) R_{i, j}^{*}+\frac{\Delta t}{\left(\Delta x^{2}\right)} D \sum_{\langle n n\rangle} R_{i^{\prime}, j^{\prime}}^{*}
$$

where the sum runs over the 4 nearest neighbours. It is shown by Von Neumann stability analysis that the solution is stable for $\frac{\Delta t}{\Delta x^{2} D}<\frac{1}{4}$.

\section{Observe}

Agents estimate the resource levels on their present location and the 4 Von Neumann nearest neighbours. The difference between the estimated and the actual amount is drawn from a normal distribution with mean 0 and standard deviation equal to the parameter $R_{\text {unc }}$

$$
R_{i, j}^{a}=R_{i, j}+N\left(0, R_{u n c}\right)
$$

with $R_{i, j}^{a}$ the estimated amount, $R_{i, j}$ the actual amount and $N\left(0, R_{u n c}\right)$ a random number drawn from a uniform distribution between 0 and $R_{u n c}$. The result of the observation is stored by the agent until it is replaced by the next observation.

\section{Harvest?}

The decision of an agent on whether to harvest from its current location is based on the current internal energy of the agent, the amount of resource that the agent estimates to be present on the site, and the amount of resource it estimates to be present on neighbouring sites. The current internal energy is compared with the energy maintenance per time-step

$$
H\left(E^{a}\right)= \begin{cases}0 & \text { if } E^{a}<E_{m} \\ \frac{E^{a}}{E_{m}}-1 & \text { if } E^{a} \geq E_{m}\end{cases}
$$

$H$ ranges between 0 and infinity. A small value of $H$ indicates that the agent has a low internal energy and needs to harvest soon (i.e. the agent is hungry). If $H=0$, 
then the agent must harvest to survive for another time-step. An agent compares perceived resource on its present site to the maximum possible harvest $R_{\max }$, which is constant

$$
G\left(R_{i, j}^{a}, n_{i, j}\right)= \begin{cases}0 & \text { if } R_{i, j}^{a} / n_{i, j}<R_{\max } \\ 1-\frac{R_{i, j}^{a}}{n_{i, j} R_{\max }} & \text { if } R_{i, j}^{a} / n_{i, j} \geq R_{\max }\end{cases}
$$

The resource that the agent expects to harvest is given by the estimated amount of resource on the patch divided by the total number of agents on the patch. $G$ ranges between 0 and 1 . If $G$ is close to 1 , then the expected harvest is small. If $G$ is close to zero, then the agent expects to harvest close to the maximum possible amount.

The resource on neighbouring sites is taken into account using the following function

$$
F\left(R_{n}\right)=\frac{R_{n}}{R_{i, j}} \frac{n_{i, j}}{n_{n}+1}
$$

with $R_{n}$ the resource at the neighbour, and $n_{n}$ the number of agents on the neighbouring site. This function estimates the amount of resource that may be harvested from the neighbouring site, relative to the amount that may be harvested from the present location. The function ranges between zero and infinity. Small values imply that more resource could be harvested from the present location, whereas large values imply that more could be harvested from the neighbouring location. The estimate is based on the assumption that the available resource will be shared with any other agents present on the respective site. The estimate is made for all of the neighbours in the Von Neumann neighbourhood. The highest value of $F\left(R_{n}\right)$ from these neighbours is used for the computation of the probability to harvest.

The final probability to harvest is computed based on the functions $H, G$, and $F$

$$
P_{\text {harvest }}^{a}=e^{-w_{h}^{a} H G F}
$$

where $w_{h}^{a}$ represents the internal tendency of the individual agent to harvest. Thus an agent is likely to harvest if it is hungry, if it sees a good opportunity for making a harvest, and if it has a high internal tendency. Since the functions $H, G$, and $F$ are dimensionless, the state variable $w_{h}^{a}$ is also dimensionless. Note that the probability of harvesting goes to one if the agent is very hungry, or if the expected harvest is equal to the maximum possible harvest.

\section{Harvest}

The harvest submodel is composed of several other submodels that carry out the action of the agent to harvest. The flowchart of the harvest submodel is in Fig. 6.14, and the submodels in this flowchart are explained below. 


\section{Select partner}

A harvesting agent checks the Von Neumann neighbourhood for the presence of any other agents that have not yet harvested. If no agents are present, the harvester cannot choose any interaction partner. If one or more agents are present, the harvester will consider its trust $\theta^{a, a^{\prime}}$ towards those agents. This trust is computed as

$\theta^{a, a^{\prime}}= \begin{cases}w_{r e p}\left(\rho^{a^{\prime}}+U\left(-\rho_{u n c}, \rho_{u n c}\right)\right)+\left(1-w_{r e p}\right) \tau^{a, a^{\prime}} & \text { if interactions remembered } \\ \rho^{a^{\prime}} & \text { if no interactions remembered }\end{cases}$

Here $\tau^{a, a^{\prime}}$ is the memory of past interactions between the agents, $\rho^{a^{\prime}}$ is the reputation of the agent $a^{\prime}$, and $U\left(-\rho_{u n c}, \rho_{u n c}\right)$ represents the uncertainty in the observation of this reputation by agent $a$, which is drawn from a uniform distribution between $-\rho_{\text {unc }}$ and $\rho_{\text {unc }}$. The size of this deviation is drawn from a uniform probability distribution $\Delta \rho^{a^{\prime}} \sim U\left(-\rho_{\text {unc }}, \rho_{\text {unc }}\right)$. The value of $\tau^{a, a^{\prime}}$ can take values between 0 and 1 . A value $\tau^{a, a^{\prime}}=0$ means that the agent has always defected in previous interactions, and $\tau^{a, a^{\prime}}=1$ means that it has always cooperated.

Besides the trust towards potential interaction partners, the harvester also considers the estimated resource $R_{i, j}^{a}$ on their sites. The agent with the highest product $\theta^{a, a^{\prime}} R_{i, j}^{a}$ is chosen as interaction partner. Note that considering trust in the decision to select a partner is an optional feature of the model. If this feature is disabled, a partner is selected randomly without considering trust.

\section{Interact?}

If an potential cooperation partner $a^{\prime}$ has been chosen by the harvester $a$, then both agents decide whether to enter an interaction with each other. The probability of entering an interaction depends on the trust towards the agent $\theta^{a, a^{\prime}}$ (Eq. 6.15), and on the estimated available resource on its patch $R_{i, j}^{a}$. For the harvester, the probability is

$$
P_{\text {interact }}= \begin{cases}\theta^{a, a^{\prime}} \frac{R_{i, j}^{a}}{R_{\max }} & \text { if } R_{i, j}^{a}<R_{\max } \\ \theta^{a, a^{\prime}} & \text { if } R_{i, j}^{a} \geq R_{\max }\end{cases}
$$

For the interaction partner, the probability is determined in the same way, switching the indices $a$ and $a^{\prime}$. If both agents decide to interact, then the interaction will proceed. The interaction will count as a harvest for both agents, and neither agent will thus be able to harvest later during the same time-step. If either or both agents decide not to interact, then the harvester will harvest individually. In this case, the interaction partner may still harvest or move later during the time-step.

Note that considering trust in the decision to interact is an optional model feature. If this feature is disabled, agents will choose to interact if $R_{i, j}^{a} \geq R_{\max }$, and will decline the interaction otherwise. 


\section{Defect?}

If both the interaction partner $a$ and the harvester $a^{\prime}$ have decided to interact, then the interaction partner will decide whether to cooperate or defect. The probability of defection is a weighted average of the trust the interaction partner has towards the harvester, as well as the internal tendency of the interaction partner to defect $w_{\text {defect }}$

$$
P_{\text {defect }}=w_{\text {reciprocity }}^{a} \theta^{a, a^{\prime}}+\left(1-w_{\text {reciprocity }}^{a}\right) w_{\text {defect }}^{a}
$$

The value of the agent parameter $w_{\text {reciprocity }}$ thus determines the relative weight of trust in the decision to defect.

Considering trust in the decision to defect is an optional feature of the model. If this feature is disabled, then the probability to defect will be given solely by the internal tendency of the interaction partner $P_{\text {defect }}=w_{\text {defect }}^{a}$.

\section{Collect resource}

The harvest is carried out by updating the amount of resource on the harvested site $i, j$ and the internal energy level of the harvesting agent. If there is an interaction partner that decided to cooperate, the resource on its site is also updated. Each agent can harvest up to a maximum of $R_{\max }$. If the amount of resource of the site is insufficient to let an agent gather this maximum amount, then the available resource is harvested. The updated amount of resource is $R_{i, j}=R_{i, j}^{*}-n_{h} R_{h}$, with $R_{i, j}^{*}$ the amount before harvesting, $n_{h}$ the number of agents that are harvesting on a site, and $R_{h}$ the harvested amount per agent

$$
R_{h}= \begin{cases}R_{i, j}^{*} & \text { if } R_{i, j}^{*}<n_{h} R_{\max } \\ R_{\max } & \text { if } R_{i, j}^{*}>n_{h} R_{\max }\end{cases}
$$

Harvesting costs energy per unit of resource collected. If agents are cooperating on a harvest, then this additional cost is lowered when making the harvest. Thus $\eta_{\text {ind }}>\eta_{\text {coop }}$, with $\eta_{\text {ind }}$ the energy cost per unit of resource for individual agents, and $\eta_{\text {coop }}$ the cost for cooperating agents. If there is only a single harvesting agent, and no interaction partner, all of the collected resource is converted with efficiency $c$ and added to the internal energy

$$
E^{a}=E^{* a}+c R_{h}-\eta_{\text {ind }} c R_{h}
$$

If there is an agent cooperating with the harvest, then both agents harvest from their own location following Eq. 6.18, and share the total harvest equally

$$
\begin{gathered}
E^{a}=E^{* a}+c\left(\frac{R_{h}^{a}+R_{h}^{a^{\prime}}}{2}\right)-\eta_{\text {coop }} c R_{h}^{a} \\
E^{a^{\prime}}=E^{* a^{\prime}}+c\left(\frac{R_{h}^{a}+R_{h}^{a^{\prime}}}{2}\right)-\eta_{\text {coop }} c R_{h}^{a^{\prime}}
\end{gathered}
$$


with $R_{h}^{a}$ the amount collected by the harvester and $R_{h}^{a^{\prime}}$ by the cooperating partner.

Interaction partners that defect do not harvest anything, and spend only the initial harvest $\operatorname{cost} E_{h}$. The total harvest is still equally shared, so that

$$
\begin{aligned}
E^{a} & =E^{* a}+\frac{c R_{h}^{a}}{2}-\eta_{i n d} c R_{h}^{a} \\
E^{a^{\prime}} & =E^{* a^{\prime}}+\frac{c R_{h}^{a}}{2}
\end{aligned}
$$

Note that the harvester $a$ pays the energy cost $\eta_{\text {ind }}$ per unit of harvest, and thus does not receive any benefits from the interaction.

\section{Update reputation and memory}

After each harvest in which the harvester interacted with an interaction partner, the reputation of the interaction partner is lowered if it defected, and increased if it cooperated

$$
\rho^{a}= \begin{cases}\left(\rho^{*, a}-1\right) \zeta+1 & \text { if the agent cooperated } \\ \rho^{*, a} \zeta & \text { if the agent defected }\end{cases}
$$

This update ensures that all the previous decisions of an agent on whether or not to interfere are implicitly represented in its reputation score. The parameter $\zeta$ determines how quick the reputation changes as a result of recent decisions. The most recent action has a weight of $1-\zeta$. The old value, which contains all previous interactions, has a weight of $\zeta$. If an agent does not interfere with harvests, then its reputation score will move towards the maximum value of 1 . If the agent does interfere, the score will move towards the minimum of zero.

The memories of the harvester about the interaction partner are updated in the same way as the reputation

$$
\tau^{a, a^{\prime}}= \begin{cases}\left(\tau^{*, a, a^{\prime}}-1\right) \zeta+1 & \text { if the agent cooperated } \\ \tau^{*, a, a^{\prime}} \zeta & \text { if the agent defected }\end{cases}
$$

Here $\tau^{a, a^{\prime}}$ is the memory score of the harvester $a$ regarding the interaction partner $a^{\prime}$, and $\tau^{*, a, a^{\prime}}$ is the memory score before the update.

\section{Move?}

When deciding whether or not to move, an agent predicts how much resource it expects to obtain at its current site and at the four nearest neighbouring sites. For this decision, the estimated resource present on the neighbouring sites is updated to take into account any changes in the resource amounts due to harvests. For its current site $i, j$ the agent expects that the resource will be shared evenly among the agents on that site

$$
E_{e x p, i, j}^{a}=\frac{c R_{i, j}^{a}}{n_{i, j}}
$$


For a neighbouring site, the agent expects that it will share the resource with the agents already present at the site. The agent also considers that it will first have to spend energy to move to a neighbouring site before harvesting there. Thus, the net expected energy gain for the neighbouring site is equal to the expected harvest minus the cost of moving to the site

$$
E_{e x p, k, l}^{a}=\frac{c R_{k, l}^{a}}{n_{k, l}}-E_{m o v e} .
$$

The expected energy gain of the current site is then compared with that of the nearest neighbour with the highest expected gain. Based on this comparison, the probability of moving is computed

$$
P_{\text {move }}=e^{-w_{m}^{a} E_{\text {exp }, i, j} / E_{\text {exp }, k, l}}
$$

Thus, an agent is likely to move if the expected energy gain is higher on a neighbouring site than on the present location. Since both the expected harvests have the dimensions of energy, the variable $w_{m}^{a}$ is dimensionless. This variable represents the differences between agents in their likeliness to move.

\section{Move}

The agent moves to the nearest neighbour with the highest value of $\frac{R_{i, j}^{a}}{n_{i, j}}$. The location of the agent is updated to the new site.

\section{Maintenance}

For each agent, the daily energy cost of maintenance is deducted from the internal energy

$$
E^{a}= \begin{cases}0 & \text { if } E^{* a}<E_{m} \\ E^{* a}-E_{m} & \text { if } E^{* a} \geq E_{m}\end{cases}
$$

If the internal energy of the agent is lower than the maintenance costs, it will become zero after paying maintenance. Because the 'pay maintenance' submodel is immediately followed by the 'die?' submodel, the agent will then die without taking any further actions.

\section{Die?}

Every time-step, agents have a probability of dying or breeding, based on their internal energy. The probability of dying is

$$
P_{\text {die }}\left(E^{a}\right)=e^{-v_{d} E^{a}}
$$

Thus, the probability of dying goes to one as $E^{a}$ goes to zero. 


\section{Die}

If an agent dies it is removed from the simulation.

\section{Breed?}

If an agent does not die, it has a probability of breeding

$$
P_{\text {breed }}\left(E^{a}\right)= \begin{cases}0 & \text { if } E^{a}<E_{b} \\ 1-e^{-v_{b}\left(E^{a}-E_{b}\right)} & \text { if } E^{a} \geq E_{b}\end{cases}
$$

The submodel determines whether the agent will breed based on this probability.

\section{Breed}

If an agent breeds, it is replaced by two new agents. The energy of the parent is split evenly among the two offspring. The values of $w_{h}^{a}$ and $w_{m}^{a}$ are inherited by the offspring with some random deviation. The deviation is drawn from a uniform distribution between ${ }^{\sim} z$ and $z$. In some cases, the resulting value of $w_{h}^{a}$ and $w_{m}^{a}$ can be negative. It is then set to zero instead. In the same way, the values of the weights $w_{\text {trust }}^{a}$ and $w_{\text {rep }}^{a}$ are also inherited.

\section{A.8 Memory update}

Agents remember previous interactions only for a limited number of time-steps. The time since the interaction between agents $a$ and $a^{\prime}$ is tracked as a state variable $h^{a, a^{\prime}}$. In the submodel, this age is increased by one. After this increase, if the time since the latest interaction exceeds the maximum memory length $h^{a, a^{\prime}}$, then the memory score is erased.

\section{B One-factor-at-a-time results for growth rate $r$}

In section 6.4 .2 we have demonstrated the effects of increasing resource availability by increasing the carrying capacity $K$. For comparison, we offer here the effects of increasing the growth rate $r$. Similar to $K$, increasing values of $r$ should cause an increase in the availability of resource. Thus, we would expect similar results to those shown in section 6.4.2. Fig. B1 shows that for increasing $r$ the population size $n$ increases approximately linearly in the model with mechanisms for cooperation. In the base model, in contrast, the population size increases for small values of $r$, but for values of $r \geq 0.3, n$ starts to decrease. For values around $r=0.8$ and larger, the population collapses and almost goes to zero. Thus, the one-factor-at-a-time analysis for $r$ shows similar results to the one for $K$.

\section{C Global sensitivity analysis}

To explore the model behaviour under a wide range of parameter settings, we perform a global sensitivity analysis, varying all model parameters simultaneously across the 


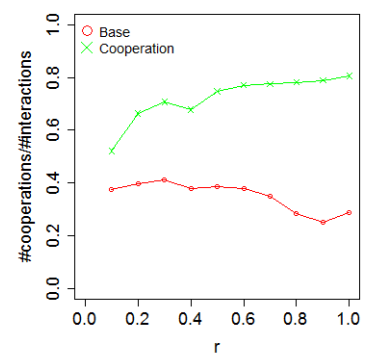

(a)

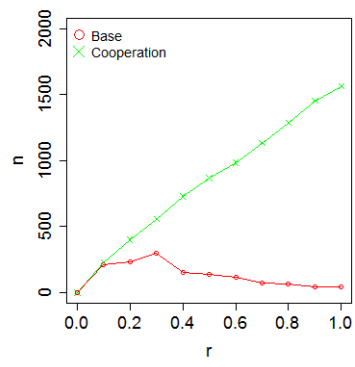

(b)

Figure B1: (a): Number of agents $n$ as function the parameter $r$. (b): Proportion of interactions that lead to cooperation, as function of the parameter $r$. In both graphs the output was averaged over time between $t=10000$ and $t=20000$.

range specified in Table 6.2. For each parameter, we sample the extremes of this range and 9 equidistant points in between these extremes. Given the large number of parameters, it is practically impossible to sample all the possible combinations of parameter values. Instead we draw 100 Latin hypercube samples, which allows us to sample a wide range of parameter values at lower computational costs. For each of the drawn sample points, model runs were performed for both the basic model, and the model with all mechanisms for cooperation enabled.

The analysis shows that the population goes extinct in a number of parameter settings (Fig. C1a). Similar tipping points, where the model behaviour switches from a positive population to extinction, are also present in a more simple version of the model (Ten Broeke, Van Voorn and Ligtenberg 2016). Given the large number of parameters, we cannot locate the tipping point in parameter space based on global sensitivity analysis. Out of 1000 sample points, in the basic model 790 runs converged to extinction, while in the model version with cooperation 722 runs converged to extinction.

Fig. C1b shows that the amount of cooperation strongly depends on the parameter settings. As would be expected, cooperation is generally less common in the base model than in the model with mechanisms to support cooperation. However, for both model versions the range of output values is wide. Even in the base model certain parameter settings lead to more than $80 \%$ of interactions ending in cooperation.

It is common practice in global sensitivity analysis to decompose the output variance into sensitivity indices attributed to the various model parameters. In our case, however, the output is far from normally distributed (Fig. C1). Thus, the variance would not be a good measure to describe the variation of the outcomes and it would not be insightful to report global sensitivity indices. In the following paragraphs, we offer more detailed investigations into the effects of specific model parameters. 


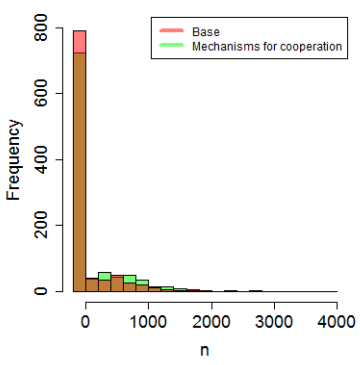

(a)

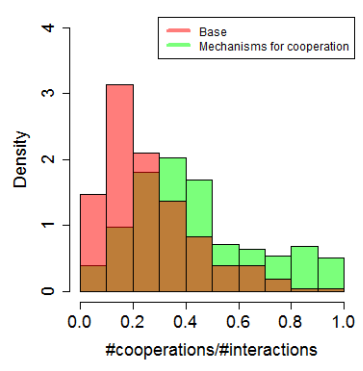

(b)

Figure C1: (a): Histogram of $n$ over all runs from the global sensitivity analysis. (b): Histogram of the proportion of interactions that lead to cooperation. The histogram was computed over all runs from the global sensitivity analysis with $n>0$. Since the total number of runs with $n>0$ is not equal for both model versions, the histogram was normalised to have a total area of 1 . 



\section{Chapter 7}

\section{General Discussion}

Agent-based models (ABMs) are popular for modelling complex adaptive systems (CAS). However, the credibility and utility of ABMs for studying CAS is hampered by a lack of available methodologies for analysing these models. It is partly due to this lack of methodologies that many studies employing ABMs do not include a systematic sensitivity analysis (Thiele et al. 2014). Thus, there is a need for sensitivity analysis methodologies that are suitable for ABMs. The main aim of this thesis is to contribute to the development of such methodologies. To this end we have evaluated the strengths and limitations of existing sensitivity analysis methods. Furthermore, we have developed new methods to deal with challenges for which existing methods were found to be insufficient.

\subsection{Aims of sensitivity analysis}

Before evaluating sensitivity analysis methodologies, it is crucial to discuss the aims that those methodologies are intended to serve. Although sensitivity analysis can serve a wide range of aims, most standard sensitivity analysis methods are designed to evaluate which parameters cause the most uncertainty in the model output. In this approach it is recommended to limit the analysis to a small number of output variables (Saltelli et al. 2008). The model parameters are then ranked according to their influence on the output variables. This approach is demonstrated in Chapters 2 (local sensitivity analysis) and 3 (global sensitivity analysis), and is commonly applied in fields like engineering or chemistry. In these fields there is usually a relatively high level of understanding of the modelled system, and a relatively low degree of uncertainty. Therefore, accurate predictions are possible. Sensitivity analysis may then help to further improve this accuracy by suggesting parameters for improved measurements.

In most ABM applications, however, improving the accuracy of model predictions is not the main aim of sensitivity analysis. ABMs are typically used to model open systems with many uncertain factors. Although prediction in these systems is not generally impossible (Helbing 2012, Troitzsch 2009), detailed predictions like in engineering systems are usually out of reach. A survey of a wide range of ABM studies has concluded that not a single one of these studies was aimed at making 
direct predictions (Heath et al. 2009). The number of ABM studies with this aim may have increased since this survey, but still appears to be relatively low. Instead, many ABMs are used to explore whether a certain conceptual model can reproduce observed behaviour of the real system (Heath et al. 2009, Macal 2016). Such an aim places entirely different demands on sensitivity analysis methodologies. Rather than to identify influential parameters, sensitivity analysis is used to answer questions such as the following:

- What kind of emergent behaviour may the model generate?

- Which model mechanisms are essential for generating this behaviour?

- To what extent is the emergent behaviour robust to parameter changes?

- What kind of behaviour is possible under different parameter regimes?

These kind of questions ask for a more qualitative kind of model assessment, which traditional sensitivity analysis methods are not designed to provide. This may explain to a large extent the short-comings of these methods for analysing ABMs.

\subsection{Evaluation of sensitivity analysis methodologies}

In this thesis a few traditional methods of sensitivity analysis have been evaluated based on their application to test-cases. Given the complexity of ABMs, and their wide range of applications, any single sensitivity analysis methodology should not be expected to be suitable for all ABM studies. As shown in Table 7.1, different sensitivity analysis methodologies have different strengths and weaknesses. Thus, the choice of sensitivity analysis methodology should depend on the types of interactions in the model, as well as the aims of the analysis. In the following paragraphs we discuss the strengths and weaknesses of each of the methods discussed in this thesis.

\subsubsection{Local sensitivity analysis}

Local sensitivity analysis was originally developed for ODE models, and has important applications for these models. For ABMs, however, its utility is limited. Local sensitivity analysis is based on partial derivatives of the model output with respect to the model parameters. For ABMs, however, there are no equations available that relate the parameters to the model outputs. As a result, the direct differential method, which is used to compute local sensitivities for ODE models, cannot be applied to ABMs. Furthermore, most ABMs contain stochastic effects. For stochastic models, the partial derivatives are not well-defined. So, local sensitivity analysis cannot be applied to ABMs without modifications. Apart from these limitations, for sensitivity analysis of ABMs it is necessary to explore a wide range of parameter changes, for example to assess the effects of interactions between parameters, or to detect tipping points. Local sensitivity analysis, in contrast, considers only small parameter changes around a single point in parameter space. Therefore, local sensitivity analysis is not suitable for assessing interaction effects or detecting tipping points in ABMs. 


\subsubsection{Model-free global sensitivity analysis}

Model-free sensitivity analysis is a form of global sensitivity analysis that is aimed at ranking model parameters based on the proportion of output variance explained by each (combination of) parameters. The method is called model-free because no regression model is used in estimating these explained variances, in contrast with regression-based sensitivity analysis (Section 7.2.3). The output variance is estimated using Monte Carlo sampling. The use of the method has been advocated for ABMs by various authors (e.g. Cariboni et al. (2007), Ligmann-Zielinska et al. (2014), Fonoberova et al. (2013)). However, our results in Chapters 3 and 4 have revealed a number of practical and conceptual issues that limit the utility of model-free sensitivity analysis for ABMs.

A practical limitation is the relatively high computational cost of the method. In Chapter 3, the method was applied to estimate sensitivity indices for 3 parameters of an ODE model describing predator-prey interaction. With a sample size of the order of $10^{4}$ model runs, significant uncertainties in the sensitivity estimates remained. Most ABMs contain a much larger number of parameters. For the relatively simple ABM discussed in Chapter 4, which contains 15 parameters, the sensitivities could not be accurately estimated even with a sample size of 17.000 model runs. Due to this inaccuracy, some sensitivity indices were estimated to be negative, which has no realistic interpretation. For these results, the sampling design suggested by Saltelli (2002) was used. Other sampling designs have been explored in Chapter 3. Some of these other designs might yield more accurate estimates, but the computational costs are expected to be extremely high.

A second issue is that whereas model-free global sensitivity analysis expresses the sensitivity indices in terms of explained variance, for many ABMs the variance is not a good measure to describe the variation in the model output. If the model output is normally distributed, then its variation is fully described by the variance. For many ABMs, such as the one discussed in Chapter 4, the output distribution is far from normal. The variance is then insufficient to describe the variation of the output. Thus, before applying model-free global sensitivity analysis, it should always be verified whether the model output is normally distributed.

A more conceptual issue is that the aggregation of model output across a region of parameter space leads to a loss of detailed information that is crucial for understanding model behaviour. ABMs generate large amounts of output data, both on the agentlevel and on the system-level. Considering only aggregated output on the systemlevel does not yield insight in the emergence of system-level properties from lowerlevel agent interactions. It may show that some parameter is influential, but not why it it influential. Therefore, considering only aggregated system-level outputs seems contradictory to the CAS approach, since CAS is a bottom-up approach in which system-level properties emerge from lower-level interactions. Given the issues described above, model-free global sensitivity analysis appears to be only of limited use for most ABM studies.

\subsubsection{Regression-based sensitivity analysis}

Similar to model-free global sensitivity analysis, regression-based sensitivity analysis expresses parameter sensitivities in terms of explained output variance. The main 
difference between both methods is that in regression-based sensitivity analysis a regression model is fitted to the ABM output. The explained output variance for each parameter is then computed based on the regression model. Thanks to the use of a regression model, regression-based sensitivity analysis is computationally cheaper than model-free sensitivity analysis. However, the method requires a regression model that yields a good fit to the model output. For ABMs finding such a regression model is typically not a straightforward task, because the relationships between the parameters and the model outputs may be complex and may change over time. For the test-case in Chapter 4, the difficulty of finding an acceptable fit proved to be a major limitation. The method also suffers from most of the limitations that were discussed above for model-free sensitivity global sensitivity analysis. Thus, if the aim is to rank the model parameters in terms of their explained variance, then regression-based sensitivity analysis may serve as a computationally cheaper alternative for model-free global sensitivity analysis, if a suitable regression model is found. Otherwise, the use of other methods is recommended.

\subsubsection{One-factor-at-a-time sensitivity analysis}

One-factor-at-a-time (OFAT) sensitivity analysis evaluates the output for a range of values for one of the parameters, while keeping all other parameters constant. The main advantage of OFAT is that it isolates the effect of individual parameters. For each parameter, OFAT shows how changes in that parameter affect the output, for example whether the output is increased or decreased, whether this relationship is linear or non-linear, and whether parameter changes may cause a tipping point. In Chapter 3, OFAT was applied to an ODE model for the purpose of tipping point detection. The results revealed the same tipping points that may be detected using bifurcation analysis. For the ABM in Chapter 4, for which bifurcation analysis is not applicable, OFAT also revealed a number of tipping points. The main limitation of OFAT is that since it considers only variations in individual parameters, it cannot be used to explore interaction effects. As a result, it may fail to detect tipping points that are only found if multiple parameters are simultaneously changed. It is therefore recommended to supplement OFAT with a global method of sensitivity analysis. Nevertheless, OFAT serves as a good starting point for analysing ABMs and detecting tipping points. Furthermore, the computational costs of OFAT are relatively low.

\subsubsection{All-but-one-at-a-time sensitivity analysis}

All-but-one-at-a-time sensitivity analysis (ABOS) is a graphical method of global sensitivity analysis that has been discussed in Chapter 3. Application of ABOS may be based on the same samples that are used for model-free global sensitivity analysis or regression-based global sensitivity analysis. In ABOS the output of those samples is plotted as a function of one parameter, while all other parameters are varied. ABOS can help to reveal the effects of individual parameters, but unlike OFAT it also explores interaction effects. A limitation of ABOS is that it is computationally expensive. This limitation becomes irrelevant if ABOS is performed in addition to regression-based global sensitivity analysis or model-free global sensitivity analysis, in 
which case it uses the same samples and thus does not impose addition computational costs. A disadvantage of ABOS is that in case of a large number of parameters it may become difficult to graphically identify the effects of individual parameters.

\subsubsection{Earth-mover's distance}

Sensitivity analysis using the earth-mover's distance is discussed in Chapter 5. In this method, probability density functions (PDF)s of the model output are compared for different model versions or settings. The earth-mover's distance is used to quantify the difference between PDFs. Using the full PDF, rather than summarising measures like the mean or variance ensures that no information is lost due to aggregating output. The PDF may be composed either of repeated model runs each measured at the same time, or of a single model run repeatedly measured over time. By comparing these two for adaptive and non-adaptive model versions, the method may be used to measure the effects of adaptation. Although in this thesis the method was used exclusively for the purpose of measuring adaptation, it could also be used as a more general sensitivity measure for the effects of other factors on the model output.

\subsection{Lessons from applying sensitivity analysis to ABMs}

An important use of the ABM test-cases in this thesis has been to explore the effects of loosening simplifying assumptions imposed by other modelling methods. For example, ODE predator-prey models assume that individuals are identical and influence each other equally. Or, game models assume that agents follow economic rationality. In some cases, such simplifying assumptions may help to reveal core mechanics of the system. In other cases, however, they are over-simplifications. ABMs generally require a lower level of simplification than other modelling methods. Therefore ABMs can be used to verify whether relevant characteristics of the system are neglected through the simplifications of other methods. For example, in economic systems factors such as emotional attachment to a business can be strongly influential (Libre et al. 2015). ABMs are suitable for modelling such factors, that are neglected in traditional economic models. Thus, ABMs can contribute to a greater understanding of the system by showing that factors that are neglected by traditional modelling methods can have a significant impact on the system.

The downside of using ABMs compared to most other modelling methods is a loss of analytic tractability. The sensitivity analysis methodologies proposed in this thesis are intended to help mitigate this downside, and increase the utility of ABMs. The ABMs discussed in this thesis serve as examples of how sensitivity analysis may be used to obtain more information from ABMs. The main topics that have been investigated using these ABMs are adaptation and resilience, and the evolution of cooperation. 


\subsubsection{Adaptation and resilience}

Resilience is currently a widely discussed topic in the context of SES. However, definitions of resilience vary strongly across the scientific literature (Grimm and Wissel 1997). Often resilience is explained in terms of a metaphorical potential landscape. Potential landscapes have been commonly used in physics, for example in classical mechanics, electrodynamics, or quantum physics. In these applications, the potential energy has a clear meaning in terms of the motion of bodies under influence from a force. For example, bodies under influence from the gravitational force are naturally attracted to each other, and tend to minimise their potential energy by moving towards each other. While the concept of potential is frequently used as a metaphor to describe the resilience of SES, it is unclear to what extent this way of thinking translates to real-life SES. For example, it is usually unclear what aspect(s) of the real-life system the potential energy would correspond to. As a result, definitions of resilience are often vague and vary strongly between applications. Furthermore, when applied to SES, thinking in terms of potential landscapes might lead to misleading insights. For example, it is implied that there exist (local) minima in terms of potential energy that the system is naturally attracted towards. For most real-life SES, however, it is not known whether there exist stable states that the system is attracted towards, or whether the system would continuously keep moving towards new states.

Thus, to apply the concept of resilience in real-life cases, there is a need to be very specific about precisely what is meant by resilience. For example, it is crucial to specify what aspects of the system should be resilient, and what kind of pressures or shocks it should be resilient to (Carpenter et al. 2001). Furthermore, it needs to be specified how the resilience of these aspects is operationalised, for example in terms of return time, or stability of some system state. Simulation models can be helpful in ensuring that the concept of resilience in SES, as well as the mechanisms underlying this resilience are properly formalised. Furthermore, it helps the user to test the logical consistence of these mechanisms. For example, adaptation is commonly suggested to be an important source of resilience in SES (Walker et al. 2004), but few studies have verified this. In Chapter 5 an $\mathrm{ABM}$ was used to examine the effects of agent adaptation on the resilience of a population of agents competing for a renewable resource. Using OFAT sensitivity analysis it was shown that the model contains tipping points that lead to extinction of the population when crossed. To examine how adaptation affects the resilience of the population against this extinction, a model version with adaptation was compared to a version that includes adaptation through natural selection. It it shown that adaptation leads to a larger population size, and makes the population more resilient to extinction. While extinction still occurs for certain parameter settings, the parameter range for which the population remains positive is increased. Although the model discussed in this thesis may be relatively abstract, it shows how simulation modelling can be used to give a measurable interpretation to the concept of resilience that may be used for real-life applications.

\subsubsection{Cooperation}

The evolution of cooperation has been studied extensively using game theory models. Such models show that indirect reciprocity may be an important mechanism to support cooperation Nowak and Sigmund (2005). However, game theory models impose 
strict assumptions. For example, benefits from cooperation are pre-defined and constant throughout the simulation. Furthermore, ecological dynamics such as changes in population size due to cooperation are usually not taken into account (Doebeli and Hauert 2005, Gotts et al. 2003). For common-pool resource systems, however, such dynamics may be crucial. For example, a high level of cooperation might lead to an increasing population size, which in turn can decrease resource availability.

ABMs provide a useful tool for including ecological dynamics, and loosening some of the assumptions of game theory models. To this end, the ABM of Chapter 5 was extended to include cooperation between agents. Using OFAT, it was shown that ecological dynamics strongly influence cooperation. For instance, in absence of indirect reciprocity, both the level of cooperation and the population size decrease. The decreased population size, however, leads to the formation of small groups. These small groups show an increased level of cooperation. Thus, due to population size effects and group formation, cooperation is maintained even in absence of any explicit mechanisms to support it. This shows that it is important to consider ecological factors in studies on evolution of cooperation. That these factors are omitted in most studies may be largely because they quickly lead to highly complex models. ABMs, when combined with suitable analysis methodologies, may be of great value to study the effect of ecological factors on the evolution of cooperation.

\subsection{Future work}

The utility of sensitivity analysis methodologies for ABMs would be further improved by the development of software to automate their application to ABMs. Currently most software for ABMs features only limited possibilities for performing a systematic sensitivity analysis. For example, whereas Netlogo (Wilensky 1999) contains some support for sensitivity analysis, it does not contain support for most designs of global sensitivity analysis. As a result, performing such an analysis is time-consuming. An important requirement for software to automate sensitivity analysis for ABMs is that a wide range of options for storing and visualising output are offered. Whereas traditional sensitivity analysis usually focuses on a small number of outputs, ABMs can generate large amounts of data each time-step, both on the system-level and on the level of individual agents. Therefore, ensuring that the different types of output data are easily stored, accessed, and visualised is even more important for ABMs than for other modelling methods. Steps towards more practical and user-friendly application of sensitivity analysis have already been made in the form of tools like MEME (Ivanyi et al. 2007) and RNetlogo (Thiele et al. 2014).

Traditional sensitivity analysis methodologies focus on examining a small number of outputs, and creating high-level summaries of the effects of each model parameter on these outputs. Agent-based models generate large amounts of output because each individual agent possesses its own values for state variables. Thus, focussing only on a few global outputs means that a lot of information is discarded before the analysis takes place (Macal 2016, Nelson 2016). Progress may be made by collecting more information on agent-level variables as part of model analysis. For example, in Chapter 6 distributions of agent-level variables were used to better understand results from OFAT sensitivity analysis. However, relationships between agent variables, or between agent variables and system-level output, were not yet examined. The de- 
velopment of methodologies to detect such relationships may help to better analyse ABMs. Standard sensitivity analysis methods that are currently available for ABMs essentially consider all the intermediate processes as a 'black box'. Including examination of these processes as part of sensitivity analysis will require a different approach towards sensitivity analysis. Tools for finding relationships in large amounts of data have become available in other fields (Nelson 2016). Although the analysis of ABMs poses its own challenges, application of methods from these fields may be a good step towards a better use of sensitivity analysis to examine causal relationships contained in ABM output. 


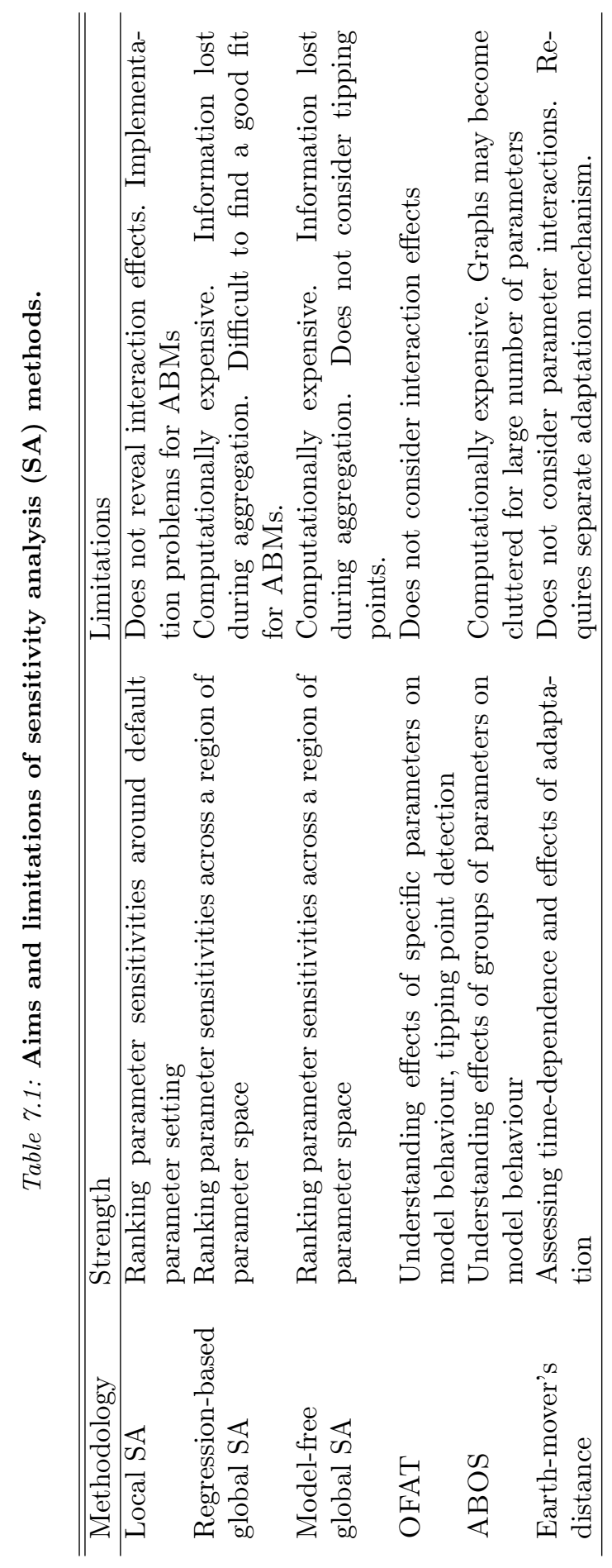





\section{References}

Allee, W. C.: 1931, Animal aggregations: A study in general sociology., University of Chicago, Chicago .

Alliance, R.: 2010, Assessing resilience in social-ecological systems: Workbook for practitioners.

Archer, G. E. B., Saltelli, A. and Sobol, I. M.: 1997, Sensitivity measures, anovalike techniques and the use of bootstrap, Journal of Statistical Computation and Simulation 58(2), 99-120.

Aubert-Kato, N., Witkowski, O. and Ikegami, T.: 2015, The hunger games: Embodied agents evolving foraging strategies on the frugal-greedy spectrum, Proceedings of the European Conference on Artificial Life pp. 357-364.

Auder, B. and Iooss, B.: 2008, Global sensitivity analysis based on entropy, Safety, reliability and risk analysis-Proceedings of the ESREL 2008 Conference, pp. 21072115.

Augusiak, J., Van den Brink, P. J. and Grimm, V.: 2014, Merging validation and evaluation of ecological models to 'evaludation': a review of terminology and a practical approach, Ecological Modelling 280, 117-128.

Axtell, R.: 1999, Why agents?: on the varied motivations for agent computing in the social sciences, Why agents? on the varied motivations for agent computing in the social sciences. In Macal, C. M. ES Sallach, D. (Eds.), Proceedings of the Workshop on Agent Simulation: Applications, Models, and Tools, Center on Social and Economic Dynamics Washington, DC.

Banks, H. T., Dediu, S. and Ernstberger, S. L.: 2007, Sensitivity functions and their uses in inverse problems, Journal of Inverse and Ill posed problems 15(7), 683.

Basurto, X., Gelcich, S. and Ostrom, E.: 2013, The social-ecological system framework as a knowledge classificatory system for benthic small-scale fisheries, Global Environmental Change 23(6), 1366-1380.

Bazykin, A. D.: 1998, Nonlinear dynamics of interacting populations, Vol. 11, World Scientific.

Bear, A. and Rand, D. G.: 2016, Intuition, deliberation, and the evolution of cooperation, Proceedings of the National Academy of Sciences 113(4), 936-941.

Bendat, J. S. and Piersol, A. G.: 2011, Random data: analysis and measurement procedures, Vol. 729, John Wiley \& Sons.

Boettiger, C., Ross, N. and Hastings, A.: 2013, Early warning signals: the charted and uncharted territories, Theoretical ecology 6(3), 255-264.

Borgonovo, E.: 2007, A new uncertainty importance measure, Reliability Engineering 83 System Safety 92(6), 771-784. 
Bousquet, F. and Le Page, C.: 2004, Multi-agent simulations and ecosystem management: a review, Ecological modelling 176(3), 313-332.

Brand, F. and Jax, K.: 2007, Focusing the meaning (s) of resilience: resilience as a descriptive concept and a boundary object, Ecology and society 12(1).

Brown, D. G., Page, S., Riolo, R., Zellner, M. and Rand, W.: 2005, Path dependence and the validation of agent-based spatial models of land use, International Journal of Geographical Information Science 19(2), 153-174.

Burgers, S. L. G. E., Hofstede, G. J., Jonker, C. M. and Verwaart, T.: 2010, Sensitivity analysis of an agent-based model of culture's consequences for trade, Progress in Artificial Economics, Springer, pp. 253-264.

Burtsev, M. and Turchin, P.: 2006, Evolution of cooperative strategies from first principles, Nature 440(7087), 1041-1044.

Cabell, J. F. and Oelofse, M.: 2012, An indicator framework for assessing agroecosystem resilience, Ecology and Society 17(1): 18.

Campolongo, F., Saltelli, A. and Cariboni, J.: 2011, From screening to quantitative sensitivity analysis. a unified approach, Computer Physics Communications $\mathbf{1 8 2}(4), 978-988$.

Cariboni, J., Gatelli, D., Liska, R. and Saltelli, A.: 2007, The role of sensitivity analysis in ecological modelling, Ecological Modelling 203(1), 167-182.

Carpenter, S., Walker, B., Anderies, J. M. and Abel, N.: 2001, From metaphor to measurement: resilience of what to what?, Ecosystems 4(8), 765-781.

Caswell, H.: 2007, Sensitivity analysis of transient population dynamics, Ecology Letters 10(1), 1-15.

Cifdaloz, O., Regmi, A., Anderies, J. M., Rodriguez, A. A. et al.: 2010, Robustness, vulnerability, and adaptive capacity in small-scale social-ecological systems: The pumpa irrigation system in nepal, Ecology and Society 15(3), 39.

Colon, C., Claessen, D. and Ghil, M.: 2015, Bifurcation analysis of an agent-based model for predator-prey interactions, Ecological Modelling 317, 93-106.

Crépin, A.-S. and Lindahl, T.: 2009, Grazing games: sharing common property resources with complex dynamics, Environmental and Resource Economics 44(1), 29 46.

Crooks, A., Castle, C. and Batty, M.: 2008, Key challenges in agent-based modelling for geo-spatial simulation, Computers, Environment and Urban Systems 32(6), 417430.

Cukier, R. I., Levine, H. B. and Shuler, K. E.: 1978, Nonlinear sensitivity analysis of multiparameter model systems, Journal of computational physics 26(1), 1-42.

Dhooge, A., Govaerts, W. and Kuznetsov, Y. A.: 2003, Matcont: a matlab package for numerical bifurcation analysis of odes, ACM Transactions on Mathematical Software (TOMS) 29(2), 141-164.

Dickinson, R. P. and Gelinas, R. J.: 1976, Sensitivity analysis of ordinary differential equation systems - a direct method, Journal of computational physics 21(2), 123143.

Diekmann, O.: 2003, A beginner's guide to adaptive dynamics, Banach Center Publications 63, 47-86.

Doebeli, M. and Hauert, C.: 2005, Models of cooperation based on the prisoner's dilemma and the snowdrift game, Ecology letters 8(7), 748-766.

Doedel, E. J. and Oldeman, B.: 2009, AUTO07P: Continuation and Bifurcation soft- 
ware for ordinary differential equations., Concordia University, Montreal, Canada.

Downing, A. S., Galic, N., Goudswaard, K. P. C., van Nes, E. H., Scheffer, M., Witte, F. and Mooij, W. M.: 2013, Was lates late? a null model for the nile perch boom in lake victoria, PloS one 8(10), e76847.

Downing, D. J., Gardner, R. H. and Hoffman, F. O.: 1985, An examination of response-surface methodologies for uncertainty analysis in assessment models, Technometrics 27(2), 151-163.

Draper, N. R. and Smith, H.: 2014, Applied regression analysis, John Wiley \& Sons.

Fagiolo, G., Moneta, A. and Windrum, P.: 2007, A critical guide to empirical validation of agent-based models in economics: methodologies, procedures, and open problems, Computational Economics 30(3), 195-226.

Fick, A.: 1855, Ueber diffusion, Annalen der Physik 170(1), 59-86.

Filatova, T., Verburg, P. H., Parker, D. C. and Stannard, C. A.: 2013, Spatial agentbased models for socio-ecological systems: challenges and prospects, Environmental Modelling 8 Software 45, 1-7.

Folke, C.: 2006, Resilience: The emergence of a perspective for social-ecological systems analyses, Glob Environ Change 16(3), 253-267.

Folke, C., Carpenter, S. R., Walker, B., Scheffer, M., Chapin, T. and Rockstrom, J.: 2010, Resilience thinking: integrating resilience, adaptability and transformability, Ecology and Society 15.

Folke, C., Carpenter, S., Walker, B., Scheffer, M., Elmqvist, T., Gunderson, L. and Holling, C. S.: 2004, Regime shifts, resilience, and biodiversity in ecosystem management, Annual Review of Ecology, Evolution, and Systematics pp. 557-581.

Fonoberova, M., Fonoberov, V. A. and Mezić, I.: 2013, Global sensitivity/uncertainty analysis for agent-based models, Reliability Engineering 8 System Safety 118, 8-17.

Gell-Mann, M.: 1994, Complex adaptive systems, in G. Cowan, D. Pines and D. Meltzer (eds), Complexity: Metaphors, Models, and Reality, Addison-Wesley, pp. 17-45.

Geritz, S. A. H., Metz, J. A. J., Kisdi, É. and Meszéna, G.: 1997, Dynamics of adaptation and evolutionary branching, Physical Review Letters 78(10), 2024.

Gilbert, N.: 2002, Varieties of emergence, Agent 2002 Conference: Social agents: ecology, exchange, and evolution, Chicago, pp. 11-12.

Gilbert, N.: 2004, Agent-based social simulation: dealing with complexity, Retrieved from: http://www.agsm.edu.au/bobm/teaching/SimSS/ABSSdealingwithcomplexity-1-1.pdf.

Gilbert, N.: 2008, Agent-based models (Quantitative Applications in the Social Sciences), Sage.

Glen, G. and Isaacs, K.: 2012, Estimating sobol sensitivity indices using correlations, Environmental Modelling $\&$ Software 37, 157-166.

Gotts, N. M., Polhill, J. G. and Law, A. N. R.: 2003, Agent-based simulation in the study of social dilemmas, Artificial Intelligence Review 19(1), 3-92.

Grazzini, J.: 2012, Analysis of the emergent properties: stationarity and ergodicity, $J$ Artif Soc Soc Simul 15(2), 7.

Griffin, A. S., West, S. A. and Buckling, A.: 2004, Cooperation and competition in pathogenic bacteria.

Grimm, V., Berger, U., Bastiansen, F., Eliassen, S., Ginot, V., Giske, J., GossCustard, J., Grand, T., Heinz, S. K., Huse, G. et al.: 2006, A standard proto- 
col for describing individual-based and agent-based models, Ecological modelling 198(1), 115-126.

Grimm, V., Berger, U., DeAngelis, D. L., Polhill, J. G., Giske, J. and Railsback, S. F.: 2010, The odd protocol: a review and first update, Ecological modelling 221(23), 2760-2768.

Grimm, V., Revilla, E., Berger, U., Jeltsch, F., Mooij, W. M., Railsback, S. F., Thulke, H.-H., Weiner, J., Wiegand, T. and DeAngelis, D. L.: 2005, Patternoriented modeling of agent-based complex systems: lessons from ecology, Science 310(5750), 987-991.

Grimm, V. and Wissel, C.: 1997, Babel, or the ecological stability discussions: an inventory and analysis of terminology and a guide for avoiding confusion, Oecologia $\mathbf{1 0 9}(3), 323-334$.

Guckenheimer, J. and Holmes, P. J.: 2013, Nonlinear oscillations, dynamical systems, and bifurcations of vector fields, Vol. 42, Springer Science \& Business Media.

Gunderson, L. H.: 2000, Ecological resilience - in theory and application, Annu. Rev. Ecol. Syst. 31, 425-439.

Gurney, W. and Nisbet, R. M.: 1998, Ecological dynamics, Oxford University Press, Oxford.

Hamby, D. M.: 1994, A review of techniques for parameter sensitivity analysis of environmental models, Environmental monitoring and assessment 32(2), 135-154.

Hardin, G.: 2009, The tragedy of the commons, Journal of Natural Resources Policy Research 1(3), 243-253.

Hastings, A.: 2004, Transients: the key to long-term ecological understanding?, Trends in Ecology 85 Evolution 19(1), 39-45.

Heath, B., Hill, R. and Ciarallo, F.: 2009, A survey of agent-based modeling practices (january 1998 to july 2008), Journal of Artificial Societies and Social Simulation 12(4), 9 .

Heckbert, S., Costanza, R., Parrott, L. et al.: 2014, Achieving sustainable societies: lessons from modelling the ancient maya, Solutions Journal 5, 55-64.

Helbing, D.: 2012, Agent-based modeling, Social self-organization, Springer, pp. 2570.

Holland, J. H.: 2002, Complex adaptive systems and spontaneous emergence, in A. Q. Curzio and M. Fortis (eds), Complexity and industrial clusters, Springer, pp. 25-34.

Holland, J. H.: 2006, Studying complex adaptive systems, Journal of Systems Science and Complexity 19(1), 1-8.

Huston, M., DeAngelis, D. and Post, W.: 1988, New computer models unify ecological theory, BioScience 38(10), 682-691.

Ivanyi, M., Bocsi, R., Gulyas, L., Kozma, V. and Legendi, R.: 2007, The multiagent simulation suite, Emergent Agents and Socialities: Social and Organizational Aspects of Intelligence pp. 8-11.

Izquierdo, L. R., Izquierdo, S. S., Galan, J. M. and Santos, J. I.: 2009, Techniques to understand computer simulations: Markov chain analysis, Journal of Artificial Societies and Social Simulation 12(1), 6.

Jakeman, A. J., Letcher, R. A. and Norton, J. P.: 2006, Ten iterative steps in development and evaluation of environmental models, Environmental Modelling $\&$ Software 21(5), 602-614.

Jansen, M. J. W.: 1999, Analysis of variance designs for model output, Computer 
Physics Communications 117(1), 35-43.

Janssen, M. A.: 2015, A behavioral perspective on the governance of common resources, Current Opinion in Environmental Sustainability 12, 1-5.

Janssen, M. A. and Carpenter, S. R.: 1999, Managing the resilience of lakes: a multiagent modeling approach, Conservation Ecology 3(2), 15.

Kaijser, A.: 2002, System building from below: institutional change in dutch water control systems, Technology and culture 43(3), 521-548.

Kendall, M. G.: 1938, A new measure of rank correlation, Biometrika 30(1/2), 81-93.

Kramer, A. M., Dennis, B., Liebhold, A. M. and Drake, J. M.: 2009, The evidence for allee effects, Population Ecology 51(3), 341-354.

Kreps, D. M., Milgrom, P., Roberts, J. and Wilson, R.: 1982, Rational cooperation in the finitely repeated prisoners' dilemma, Journal of Economic theory 27(2), 245252.

Kümmerli, R., Gardner, A., West, S. A. and Griffin, A. S.: 2009, Limited dispersal, budding dispersal, and cooperation: an experimental study, Evolution 63(4), 939949.

Kuznetsov, Y. A.: 2004, Elements of applied bifurcation theory, Vol. 112, Springer Science \& Business Media.

Lansing, J. S.: 2003, Complex adaptive systems, Annual review of anthropology 32(1), 183-204.

Leamer, E. E.: 1983, Let's take the con out of econometrics, The American Economic Review 73(1), 31-43.

Leamer, E. E.: 2010, Tantalus on the road to asymptopia, The Journal of Economic Perspectives 24(2), 31-46.

Levin, S. A.: 1998, Ecosystems and the biosphere as complex adaptive systems, Ecosystems 1(5), 431-436.

Levin, S. A.: 2003, Complex adaptive systems: exploring the known, the unknown and the unknowable, Bulletin of the American Mathematical Society 40(1), 3-19.

Levin, S. A., Xepapadeas, T., Crépin, A.-S., Norberg, J., De Zeeuw, A., Folke, C., Hughes, T., Arrow, K., Barrett, S., Daily, G. et al.: 2013, Social-ecological systems as complex adaptive systems: modeling and policy implications, Environment and Development Economics 18(02), 111-132.

Libre, S. V. D., van Voorn, G. A. K., ten Broeke, Guus, A., Bailey, M., Berentsen, P. and Bush, S. R.: 2015, Effects of social factors on fishing effort: The case of the philippine tuna purse seine fishery, Fisheries Research 172, 250-260.

Ligmann-Zielinska, A., Kramer, D. B., Cheruvelil, K. S. and Soranno, P. A.: 2014, Using uncertainty and sensitivity analyses in socioecological agent-based models to improve their analytical performance and policy relevance, PloS one $\mathbf{9}(10)$, e109779.

Lindgren, K.: 1992, Evolutionary phenomena in simple dynamics, Artificial life II, pp. $295-312$.

Ling, H. and Okada, K.: 2007, An efficient earth mover's distance algorithm for robust histogram comparison, IEEE transactions on pattern analysis and machine intelligence 29(5), 840-853.

Liu, H., Chen, W. and Sudjianto, A.: 2006, Relative entropy based method for probabilistic sensitivity analysis in engineering design, Journal of Mechanical Design $\mathbf{1 2 8}(2), 326-336$.

Lorscheid, I., Heine, B.-O. and Meyer, M.: 2012, Opening the 'black box'of simu- 
lations: increased transparency and effective communication through the systematic design of experiments, Computational and Mathematical Organization Theory 18(1), 22-62.

Louzoun, Y. and Solomon, S.: 2004, Proliferation and competition in discrete biological systems, New Directions in Statistical Physics, Springer, pp. 225-242.

Macal, C. M.: 2016, Everything you need to know about agent-based modelling and simulation, Journal of Simulation 10(2), 144-156.

Macal, C. M. and North, M. J.: 2009, Agent-based modeling and simulation, Winter simulation conference, Winter simulation conference, pp. 86-98.

Macal, C. M. and North, M. J.: 2010, Tutorial on agent-based modelling and simulation, Journal of simulation 4(3), 151-162.

Mann, H. B.: 1945, Nonparametric tests against trend, Econometrica: Journal of the Econometric Society pp. 245-259.

Mao, A., Dworkin, L., Suri, S. and Watts, D. J.: 2017, Resilient cooperators stabilize long-run cooperation in the finitely repeated prisoner's dilemma, Nature communications 8, 13800 .

Mara, T. A. and Tarantola, S.: 2012, Variance-based sensitivity indices for models with dependent inputs, Reliability Engineering \& System Safety 107, 115-121.

Matthews, R. B., Gilbert, N. G., Roach, A., Polhill, J. G. and Gotts, N. M.: 2007, Agent-based land-use models: a review of applications, Landscape Ecology 22(10), 1447-1459.

McCauley, E., Nisbet, R. M., Murdoch, W. W., de Roos, A. M. and Gurney, W. S. C.: 1999, Large-amplitude cycles of daphnia and its algal prey in enriched environments, Nature 402(6762), 653-656.

Milich, L.: 1999, Resource mismanagement versus sustainable livelihoods: The collapse of the newfoundland cod fishery, Society \& Natural Resources 12(7), 625-642.

Milinski, M., Semmann, D. and Krambeck, H.-J.: 2002, Reputation helps solve the 'tragedy of the commons', Nature 415(6870), 424-426.

Mood, A., Graybill, F. A. and Boes, D. C.: 1974, Introduction to the theory of statistics, McGraw-Hill.

Nair, A., Narasimhan, R. and Choi, T. Y.: 2009, Supply networks as a complex adaptive system: Toward simulation-based theory building on evolutionary decision making, Decision Sciences 40(4), 783-815.

Nelson, B. L.: 2016, 'some tactical problems in digital simulation' for the next 10 years, Journal of Simulation 10(1), 2-11.

Nelson, D. R.: 2011, Adaptation and resilience: responding to a changing climate, Wiley Interdisciplinary Reviews: Climate Change 2(1), 113-120.

Nowak, M. A.: 2006, Five rules for the evolution of cooperation, science 314(5805), 1560-1563.

Nowak, M. A. and Sigmund, K.: 1998, Evolution of indirect reciprocity by image scoring, Nature 393(6685), 573-577.

Nowak, M. A. and Sigmund, K.: 2005, Evolution of indirect reciprocity, Nature 437, 1291-1298.

Organization, W. M.: 2006, Guide to hydrological practices, volume II, management of water resources and application of hydrological practices (6th Ed.)., WMO, Geneva, Switzerland.

Ostrom, E.: 2015, Governing the commons, Cambridge university press. 
Ostrom, E., Burger, J., Field, C. B., Norgaard, R. B. and Policansky, D.: 1999, Revisiting the commons: local lessons, global challenges, science 284(5412), 278 282.

Ostrom, E. and Walker, J.: 2004, Trust and reciprocity, volume vi of russel sage foundation series on trust, Russel Sage Foundation, New York .

Parker, D. C., Manson, S. M., Janssen, M. A., Hoffmann, M. J. and Deadman, P.: 2003, Multi-agent systems for the simulation of land-use and land-cover change: a review, Annals of the association of American Geographers 93(2), 314-337.

Phillips, P. C. B. and Xiao, Z.: 1998, A primer on unit root testing, Journal of Economic Surveys 12(5), 423-470.

R Core Team: 2012, A language and environment for statistical computing. vienna, austria: $\mathrm{R}$ foundation for statistical computing.

Railsback, S. F.: 2001, Concepts from complex adaptive systems as a framework for individual-based modelling, Ecological modelling 139(1), 47-62.

Railsback, S. F. and Grimm, V.: 2011, Agent-based and individual-based modeling: a practical introduction, Princeton university press.

Rakovec, O., Hill, M. C., Clark, M. P., Weerts, A. H., Teuling, A. J. and Uijlenhoet, R.: 2014, Distributed evaluation of local sensitivity analysis (delsa), with application to hydrologic models, Water Resources Research 50(1), 409-426.

Rammel, C., Stagl, S. and Wilfing, H.: 2007, Managing complex adaptive systems-a co-evolutionary perspective on natural resource management, Ecol. Econ. 63(1), 921.

Refsgaard, J. C. and Henriksen, H. J.: 2004, Modelling guidelines-terminology and guiding principles, Advances in Water Resources 27(1), 71-82.

Richard, R., Casas, J. and McCauley, E.: 2015, Sensitivity analysis of continuous-time models for ecological and evolutionary theories, Theoretical Ecology 8(4), 481-490.

Ricker, W. E.: 1954, Stock and recruitment, Journal of the Fisheries Board of Canada 11(5), 559-623.

Rosenzweig, M. L.: 1971, Paradox of enrichment: destabilization of exploitation ecosystems in ecological time, Science 171(3969), 385-387.

Rosenzweig, M. L. and MacArthur, R. H.: 1963, Graphical representation and stability conditions of predator-prey interactions, American Naturalist pp. 209-223.

Rubner, Y., Tomasi, C. and Guibas, L. J.: 1998, A metric for distributions with applications to image databases, Computer Vision, 1998. Sixth International Conference on, IEEE, pp. 59-66.

Rykiel, E. J.: 1996, Testing ecological models: the meaning of validation, Ecological modelling 90(3), 229-244.

Saltelli, A.: 2002, Making best use of model evaluations to compute sensitivity indices, Computer Physics Communications 145(2), 280-297.

Saltelli, A., Annoni, P., Azzini, I., Campolongo, F., Ratto, M. and Tarantola, S.: 2010, Variance based sensitivity analysis of model output. design and estimator for the total sensitivity index, Computer Physics Communications 181(2), 259-270.

Saltelli, A., Ratto, M., Andres, T., Campolongo, F., Cariboni, J., Gatelli, D., Saisana, M. and Tarantola, S.: 2008, Global sensitivity analysis: the primer, Wiley.

Saltelli, A., Tarantola, S., Campolongo, F. and Ratto, M.: 2004a, Sensitivity analysis in practice: a guide to assessing scientific models, John Wiley \& Sons.

Saltelli, A., Tarantola, S., Campolongo, F. and Ratto, M.: 2004b, Sensitivity analysis 
in practice: a guide to assessing scientific models, John Wiley \& Sons.

Scheffer, M.: 2009, Critical transitions in nature and society, Princeton University Press.

Scheffer, M., Bascompte, J., Brock, W. A., Brovkin, V., Carpenter, S. R., Dakos, V., Held, H., Van Nes, E. H., Rietkerk, M. and Sugihara, G.: 2009, Early-warning signals for critical transitions, Nature 461(7260), 53-59.

Scheffer, M. and Carpenter, S. R.: 2003, Catastrophic regime shifts in ecosystems: linking theory to observation, Trends in ecology \& evolution 18(12), 648-656.

Scheffer, M., Carpenter, S. R., Lenton, T. M., Bascompte, J., Brock, W., Dakos, V., Van de Koppel, J., Van de Leemput, I. A., Levin, S. A., Van Nes, E., Pascual, M. and Vandermeer, J.: 2012, Anticipating critical transitions, Science 338, 344-348.

Scheffer, M., Rinaldi, S., Kuznetsov, Y. and Van Nes, E.: 1997, Seasonal dynamics of daphnia and algae explained as a periodically forced predator-prey system, Oikos pp. 519-532.

Schelling, T. C.: 1971, Dynamic models of segregation, Journal of mathematical sociology 1(2), 143-186.

Schlueter, M., McAllister, R. R. J., Arlinghaus, R., Bunnefeld, N., Eisenack, K., Hoelker, F., Milner-gulland, E. J., Müller, B., Nicholson, E., Quaas, M. et al.: 2012, New horizons for managing the environment: a review of coupled social-ecological systems modeling, Natural Resource Modeling 25(1), 219-272.

Schlüter, M. and Pahl-Wostl, C.: 2007, Mechanisms of resilience in common-pool resource management systems: an agent-based model of water use in a river basin, Ecology and Society 12(2), 4.

Schmolke, A., Thorbek, P., DeAngelis, D. L. and Grimm, V.: 2010, Ecological models supporting environmental decision making: a strategy for the future, Trends in ecology \& evolution 25(8), 479-486.

Schouten, M., Opdam, P., Polman, N. and Westerhof, E.: 2013, Resilience-based governance in rural landscapes: experiments with agri-environment schemes using a spatially explicit agent-based model, Land Use Policy 30(1), 934-943.

Seydel, R.: 2010, Practical bifurcation and stability analysis, Vol. 5, Springer Science and Business Media.

Shampine, L. F. and Reichelt, M. W.: 1997, The matlab ode suite, SIAM journal on scientific computing 18(1), 1-22.

Sobol', I. M.: 2001, Global sensitivity indices for nonlinear mathematical models and their monte carlo estimates, Mathematics and computers in simulation 55(1), 271280.

Sobol', I. M. and Kucherenko, S.: 2009, Derivative based global sensitivity measures and their link with global sensitivity indices, Mathematics and Computers in Simulation 79(10), 3009-3017.

Stauffer, D. and Solomon, S.: 2007, Ising, schelling and self-organising segregation, The European Physical Journal B 57(4), 473-479.

Stephens, P. A., Sutherland, W. J. and Freckleton, R. P.: 1999, What is the allee effect?, Oikos pp. 185-190.

Swinscow, T. D. V.: 1997, Statistics at square one, 9th edn. revised by campbell mj, university of southampton.

Tarantola, S., Gatelli, D. and Mara, T. A.: 2006, Random balance designs for the estimation of first order global sensitivity indices, Reliability Engineering 85 System 
Safety 91(6), 717-727.

Taylor, C. M. and Hastings, A.: 2005, Allee effects in biological invasions, Ecology Letters 8(8), 895-908.

Ten Broeke, G. A., Van Voorn, G. A. K., Kooi, B. W. and Molenaar, J.: 2016, Detecting tipping points in ecological models with sensitivity analysis, Mathematical Models of Natural Phenomena 11(4), 47-72.

Ten Broeke, G. A., Van Voorn, G. A. K. and Ligtenberg, A.: 2016, Which sensitivity analysis method should i use for my agent-based model?, Journal of Artificial Societies and Social Simulation 19 (1).

Ten Broeke, G. A., Van Voorn, G. A. K. and Molenaar, J.: 2017, Resilience through adaptation, PLoS One 12(2), e0171833.

Tesfatsion, L.: 2003, Agent-based computational economics: modeling economies as complex adaptive systems, Information Sciences 149(4), 262-268.

Thiele, J. C., Kurth, W. and Grimm, V.: 2014, Facilitating parameter estimation and sensitivity analysis of agent-based models: A cookbook using netlogo and r, Journal of Artificial Societies and Social Simulation 17(3), 11.

Troitzsch, K. G.: 2009, Not all explanations predict satisfactorily, and not all good predictions explain, Journal of Artificial Societies and Social Simulation 12(1), 10.

van Lith, J.: 2001, Ergodic theory, interpretations of probability and the foundations of statistical mechanics, Studies in History and Philosophy of Science Part B: Studies in History and Philosophy of Modern Physics 32(4), 581-594.

van Nes, E. H. and Scheffer, M.: 2003, Alternative attractors may boost uncertainty and sensitivity in ecological models, Ecological Modelling 159(2), 117-124.

Van Nes, E. H. and Scheffer, M.: 2005, A strategy to improve the contribution of complex simulation models to ecological theory, Ecological modelling 185(2), 153164.

Van Voorn, G. A. K., Hemerik, L., Boer, M. P. and Kooi, B. W.: 2007, Heteroclinic orbits indicate overexploitation in predator-prey systems with a strong allee effect, Mathematical biosciences 209(2), 451-469.

Van Voorn, G. A. K., Kooi, B. W. and Boer, M. P.: 2010, Ecological consequences of global bifurcations in some food chain models, Mathematical biosciences 226(2), 120-133.

van Voorn, G. A. K., Verburg, R. W., Kunseler, E. M., Vader, J. and Janssen, P. H. M.: 2016, A checklist for model credibility, salience, and legitimacy to improve information transfer in environmental policy assessments, Environmental Modelling E Software 83, 224-236.

Varma, A., Morbidelli, M. and Wu, H.: 1999, Parametric Sensitivity in Chemical Systems., Cambridge: Cambridge Series in Chemical Engineering.

Vemer, P., Van Voorn, G. A. K., Corro Ramos, I., Krabbe, P. F. M., Al, M. J. and Feenstra, T. L.: 2013, Improving model validation in health technology assessment: Comments on guidelines of the ispor-smdm modeling good research practices task force., Value in Health. 16, 1106-1107.

Venkatasubramanian, S.: 2013, Moving heaven and earth: Distances between distributions, ACM SIGACT News 44(3), 56-68.

Wald, A. and Wolfowitz, J.: 1940, On a test whether two samples are from the same population, The Annals of Mathematical Statistics 11(2), 147-162.

Walker, B., Holling, C. S., Carpenter, S. and Kinzig, A.: 2004, Resilience, adaptability 
and transformability in social-ecological systems, Ecology and society $\mathbf{9}(2)$.

West, S. A., Griffin, A. S. and Gardner, A.: 2007, Evolutionary explanations for cooperation, Current Biology 17(16), R661-R672.

Wiggins, S.: 1990, Introduction to applied nonlinear dynamical systems and chaos, Vol. 2, Springer Science \& Business Media.

Wilensky, U.: 1997, Netlogo segregation model [computer model]. center for connected learning and computer-based modeling. retrieved from: http://ccl.northwestern.edu/netlogo/models/segregation, Northwestern University, Evanston, IL .

Wilensky, U.: 1999, Netlogo [software]. center for connected learning and computerbased modeling. available from: http://ccl.northwestern.edu/netlogo/, Northwestern University, Evanston, IL .

Windrum, P., Fagiolo, G. and Moneta, A.: 2007, Empirical validation of agent-based models: Alternatives and prospects, Journal of Artificial Societies and Social Simulation 10(2), 8 .

Yue, S., Pilon, P., Phinney, B. and Cavadias, G.: 2002, The influence of autocorrelation on the ability to detect trend in hydrological series, Hydrological Processes 16(9), 1807-1829. 


\section{Summary}

Many human and natural systems are highly complex, because they consist of many interacting parts. Such systems are known as complex adaptive systems (CAS). Understanding CAS is possible only by studying the interactions between constituent parts, rather than focussing only on the properties of the parts in isolation. Often, the possibilities for systematically studying these interactions in real-life systems are limited. Simulation models can then be an important tool for testing what properties may emerge, given various assumptions on the interactions in the system. Agent-based models (ABMs) are particularly useful for studying CAS, because ABMs explicitly model interactions between autonomous agents and their environment.

Currently, the utility of ABMs is limited by a lack of available methodologies for analysing their results. The main tool for analysing CAS models is sensitivity analysis. Yet, standard methods of sensitivity analysis are not well-suited to deal with the the complexity of ABMs. Thus, there is a need for sensitivity analysis methodologies that are specifically developed for analysing ABMs. The objective of this thesis is to contribute such methodologies. Specifically, we propose methodologies for (1) detecting tipping points, (2) analysing the effects of agent adaptation, and (3) analysing resilience of ABMs.

Chapter 2 introduces traditional methods of sensitivity analysis. These methods are demonstrated by applying them to rank the most influential parameters of an ODE model of predator-prey interaction. Furthermore, the role of sensitivity analysis in model validation is discussed.

In Chapter 3 we investigate the use of sensitivity analysis for detecting tipping points. Whereas bifurcation analysis methods are available for detecting tipping points in ODE models, these methods are not applicable to ABMs. Therefore, we use an ODE model to verify the results from sensitivity analysis against those of bifurcation analysis. We conclude that one-factor-at-a-time sensitivity analysis (OFAT) is a helpful method for detecting tipping points. However, OFAT is a local method that considers only changes in individual parameters. It is therefore recommended to supplement OFAT with a global method to investigate interaction effects. For this purpose, we recommend all-but-one-at-a-time sensitivity analysis (ABOS) as a graphical sensitivity analysis method that takes into account parameter interactions and can help with the detection of tipping points.

In Chapter 4 we introduce a basic ABM model of agents competing in a spatial environment for a renewable resource. This basic model will be extended in the subsequent chapters, and will serve as a testing case for various sensitivity analysis methods. In Chapter 4, it is used to assess the utility of existing sensitivity analysis 
methods for ABMs. The results show that traditional methods of sensitivity are not sufficient to analyse the ABM, due to the presence of tipping points and other strong non-linearities in the model output. In contrast, OFAT is found to be helpful for detecting tipping points, as was suggested in Chapter 3. Based on these outcomes, OFAT is recommended as a starting point for sensitivity analyis of ABMs, preferably supplemented by a global method to investigate interaction effects.

In Chapter 5 we extend the ABM of Chapter 4 by adding agent adaptation in the form of a mechanism of natural selection. On short time-scales, the model behaviour appears to be similar to the non-adaptive model version. On longer time-scales, the agent adaptation causes the state of the model to gradually change as agents continue to adapt to their surroundings. We propose a sensitivity analysis method to measure the effects of this adaptation. This method is based on a quantification of the difference between probability density functions of model version with and without adaptation. Using this method, we show that this adaptation increases the resilience of the system by giving it the flexibility needed to respond to pressures.

In Chapter 6 we further extend the test-case by giving agents the option to harvest either cooperatively or individually. Cooperation increases the potential yields, but introduces the risk of defection of the interaction partner. It is shown that ecological factors, which are usually not considered in models on cooperation, strongly affect the level of cooperation in the system. For example, low levels of cooperation lead to a decreased population size, and causes the formation of small groups of agents with a higher level of cooperation. As a result, cooperation persists even without any mechanisms to promote it. Nevertheless, the inclusion of such mechanisms in the form of indirect reciprocity does further increase the level of cooperation. Furthermore, we show that the resulting high levels of cooperation, depending on the circumstances, can increase the resilience of the agent population against shocks.

To conclude, in this thesis several methodologies have been proposed to help with ABM analysis. Specifically, OFAT and ABOS are recommended for detecting tipping points in ABMs, and in Chapter 5 a protocol is introduced for quantifying the effects of adaptation. By suggesting these methodologies, this thesis aims to contribute to the utility of ABMs, especially for studying CAS. 


\section{Samenvatting}

Veel menselijke en natuurlijke systemen zijn complex door de aanwezigheid van een groot aantal interacterende componenten. Zulke systemen staan bekend als complex adaptieve systemen (CAS). Het begrijpen van CAS vereist studie van de interacties tussen de componenten. Vaak zijn de mogelijkheden om deze interacties in echte systemen te bestuderen gelimiteerd. Simulatiemodellen zijn dan een belangrijk hulpmiddel om het systeem beter te begrijpen. Vooral agent-gebaseerde modellen (ABMs) zijn geschikt om CAS te bestuderen, omdat in ABMs expliciet de interacties tussen autonome agenten en hun omgeving wordt gemodelleerd.

Momenteel wordt de bruikbaarheid van ABMs beperkt door een gebrek aan beschikbare methodes om de resultaten van deze modellen te analyseren. De belangrijkste methode voor de analyse van ABMs is gevoeligheidsanalyse. Standaard methodes van gevoeligheidsanalyse kunnen echter niet goed omgaan met de complexiteit van ABMs. Daarom zijn methodes van gevoeligheidsanalyse nodig die zijn ontwikkeld voor de analyse van ABMs. Het doel van deze these is het leveren van zulke methodes. In de these worden methodes voorgesteld voor (1) detectie van kantelpunten, (2) analyse van de effecten van adaptatie, en (3) analyse van de veerkracht van ABMs.

In Hoofdstuk 2 worden bestaande methodes van gevoeligheidsanalyse geintroduceerd. Deze methodes worden gedemonstreerd door ze te gebruiken om de meest invloedrijke parameters te bepalen van een ODE predator-prooi model. Daarnaast wordt de rol van gevoeligheidsanalyse voor modelvalidatie besproken.

In Hoofdstuk 3 wordt het gebruik van gevoeligheidsanalyse voor detectie van kantelpunten onderzocht. Bifurcatie-analyse wordt gebruikt voor de detectie van kantelpunten in ODE modellen, maar is niet toepasbaar op ABMs. Daarom gebruiken we een ODE model om de resultaten van gevoeligheidsanalyse te vergelijken met die van bifurcatieanalyse. We concluderen dat one-factor-at-a-time gevoeligheidsanalyse (OFAT) een bruikbare methode is voor detectie van kantelpunten. Maar, OFAT is een lokale methode die alleen veranderingen in afzonderlijke parameters beschouwt. Het wordt daarom aangeraden om OFAT aan te vullen met een globale methode om interactie-effecten te onderzoeken. Voor dit doeleinde raden we all-but-one-at-a-time gevoeligheidsanalyse (ABOS) aan. ABOS is een visuele methode die interacties in beschouwing neemt, en die kan helpen bij de detectie van kantelpunten.

In Hoofdstuk 4 introduceren we een ABM waarin agenten in een ruimtelijke omgeving concurreren om een hernieuwbare grondstof te verzamelen. Dit model wordt in de daaropvolgende hoofdstukken uitgebreid, en wordt gebruikt als een testmodel voor methodes van gevoeligheidsanalyse. In Hoofdstuk 4 wordt het model gebruikt 
om de bruikbaarheid van bestaande methodes van gevoeligheidsanalyse voor de analyse van ABMs te demonstreren. De resultaten tonen aan dat de bestaande methodes niet voldoen voor de analyse van ABMs, omdat deze niet goed omgaan met kantelpunten en andere sterke niet-lineariteiten. OFAT blijkt wel bruikbaar voor de detectie van kantelpunten, zoals eerder in Hoofdstuk 3 werd voorgesteld. Daarom wordt OFAT aangeraden als startpunt voor de gevoeligheidsanalyse van ABMs, bij voorkeur aangevuld door een globale methode van gevoeligheidsanalyse om interactieeffecten te onderzoeken.

In Hoofdstuk 5 wordt aan het ABM van Hoofdstuk 4 adaptatie toegevoegd. Deze adaptie vindt plaats via natuurlijke selectie. Op korte tijdschalen lijkt het modelgedrag vergelijkbaar met de niet-adaptieve versie. Op langere tijdschalen veroorzaakt de adaptie een geleidelijke verandering in de toestand van het model. We stellen een methode van gevoeligheidsanalyse voor om dit effect van adaptatie te meten. De methode is gebaseerd op een kwantificatie van het verschil tussen de waarschijnlijkheidsverdelingen van beide modelversies. Met deze methode laten we zien dat de adaptatie de veerkracht van het systeem positief beïnvloedt. Dit komt omdat adaptatie het systeem de benodigde flexibiliteit geeft om te reageren op verschillende soorten druk.

In Hoofdstuk 6 breiden we het testmodel verder uit door agenten de keuze te geven om individueel of in samenwerking te oogsten. Samenwerking vergroot de potentiele opbrengst, maar leidt tot een risico van defectie. Het wordt aangetoond dat ecologische factoren, die meestal niet worden beschouwd in modellen over samenwerking, het niveau van samenwerking sterk beïnvloeden. Een laag niveau van samenwerking leidt bijvoorbeeld tot een afname in de omvang van de populatie, en tot het ontstaan van kleine groepen van agenten met een verhoogd niveau van samenwerking. Dit leidt ertoe dat samenwerking blijft bestaan, zelfs zonder mechanisme om het te promoten. Wel leidt de toevoeging van zulke mechanismen tot een verdere toename van samenwerking. Daarnaast laten we zien dat een hoog niveau van samenwerking de veerkracht van de populatie tegen schokken kan verhogen.

Ter conclusie, in deze these worden enkele methodes van gevoeligheidsanalyse voorgesteld voor de analyse van ABMs. OFAT en ABOS worden aangeraden voor de detectie van kantelpunten in ABMs. In Hoofdstuk 5 wordt een protocol geïntroduceerd om adaptatie te kwantificeren. Deze methoden hebben als doel om bij te dragen aan de bruikbaarheid van ABMs voor de studie van CAS. 


\section{Acknowledgements}

To begin with, I thank my supervisors for giving me the opportunity to work on this project, the support they have given me, and their contributions to this thesis. I cannot imagine this project without the contributions of George van Voorn. Aside from his contributions to the chapters in this thesis, I also thank him for all his efforts in guiding the start of my scientific career. Jaap Molenaar and Arend Ligtenberg also made many contributions to the thesis. Furthermore, were always willing to discuss ideas, and their expertise and enthusiasm during those discussions has been a great encouragement for me. I am thankful for everything I have learned from working with this supervisory team.

A few other people have made direct contributions to chapters in this thesis. Lia Hemerik gave me the idea for and worked with me on the second chapter. I also thank Egbert van Nes, who coded the software described in the second chapter, and suggested changes to an earlier version of the text. Bob Kooi made extensive contributions to the third chapter, both in terms of ideas and calculations. I am very grateful to these people for taking the time to share their expertise, and for being pleasant people to work with.

I thank the people whom I have worked with within the Wageningen University strategic programme on Complex Adaptive Systems. Arnold Bregt was involved in many of the discussions related to my project, and provided me with valuable advice throughout the project, always after first inquiring about my personal well-being. After my first presentation at a scientific conference, he convinced me to write a paper related to that presentation, which led to the publication of my first scientific paper (Chapter 4 of this thesis). It was always a pleasure to discuss ideas and spend time with my fellow PhD candidates Yang, Anouschka, Francine, Floor, and Iris. In my first year on this project, the course by Gert Jan Hofstede, Mark Kramer, and Sjoukje Osinga was very helpful for getting me started in a field that was still new for me. I also thank Martha Bakker, Tim Verwaart, Matthijs Danes, Erika Speelman, Bas Kemp, and Wim Heijman for their contributions during discussions

I thanks my colleagues at Biometris. A positive environment is very important for me to function well at work. I also enjoyed the social events with my fellow $\mathrm{PhD}$ students and other colleagues within the group. For this, I thank among others, Martin, Daniela, Nadia, Amber, Dominique, Vincent, George, Stefan, Chaozhi, Rik, Rianne J., Rianne van B., Trynstje, Cassandra, Sanne, Jeroen, Xu Dan, Li Wenhao, ... . I also thank Dinie Verbeek and Hanneke van Ommeren for their help with administrative matters. A special thanks goes to my running partners at Biometris, George and Julio. Our shared runs, sometimes through pouring rains, always helped 
me to stay calm and focused.

Aside from my colleagues, I have received a lot of encouragement from people in my personal surroundings. In that context, I thank Jaap, Marita, and all of the people at Klein Heideveld: Marcel, Valerio, Vincent, Evert, Anna, Joshua, Niteen and Sandiyah. The house has always been a good place for me to rest, and to share experiences. Tim, Nick, Joris, Luc, Samuel, Gijs, and Jasper have been close friends that added joy and meaning to my life. Lastly, I don't think I would have been able to accomplish the work on this thesis without the unconditional support from my family. I thank all of my family members, especially my parents, my sisters, and Stella. 


\section{About the author}

Guus ten Broeke was born on December 6, 1985 in Beverwijk, the Netherlands. In 2002, he completed his secondary school education at Murmellius Gymnasium, Alkmaar. He studied at the University of Amsterdam, where he completed a BSc degree in Physics \& Astronomy in 2009. For his BSc thesis, he worked on the theoretical description of vertical water flows in oceans that occur due to diffusion of temperature and salinity. In 2012, he completed his MSc degree in Physics (theoretical track), also at the University of Amsterdam. For his MSc thesis, he modelled the spontaneous formation of viral capsids from their constituent proteins based on thermodynamics. In 2013, he started his $\mathrm{PhD}$ research at Biometris (Wageningen University), which led to the completion of this thesis. He is currently employed at Biometris as a Postdoc researcher. 



\section{List of publications}

G.A. ten Broeke, G.A.K. van Voorn, B.W. Kooi, J. Molenaar. "Detecting Tipping points with Sensitivity Analysis|Detecting Tipping points in Ecological Models with Sensitivity Analysis". Mathematical Modelling of Natural Phenomena 11(4): pp. $47-72$.

G.A. ten Broeke, G.A.K. van Voorn, A. Ligtenberg. "Which sensitivity analysis method should I use for my Agent-based Model?". Journal of Artificial Societies and Social Simulation, 19(1).

G.A. ten Broeke, G.A.K. van Voorn, A. Ligtenberg, J. Molenaar. "Resilience through adaptation". PlosOne, 12(2):e0171833.

G.A. ten Broeke, G.A.K. van Voorn, A. Ligtenberg, J. Molenaar. "Cooperation can improve the resilience of common-pool resource systems against over-harvesting". Under review.

S.V.D. Libre, G.A.K. van Voorn, G.A. ten Broeke, Bailey, M., Berentsen, P., \& Bush, S. R. "Effects of social factors on fishing effort: The case of the Philippine tuna purse seine fishery". Fisheries Research 172 (2015): 250-260. 


\section{PE\&RC Training and Education Statement}

With the training and education activities listed below the PhD candidate has complied with the requirements set by the C.T. de Wit Graduate School for Production Ecology and Resource Conservation (PE\&RC) which comprises of a minimum total of 32 ECTS (= 22 weeks of activities)

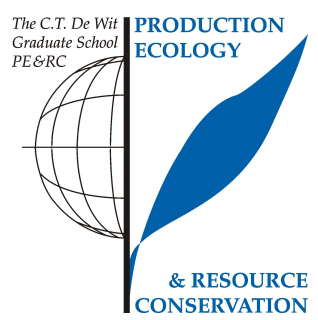

\section{Review of literature (4.5 ECTS)}

- Sensitivity analysis of agent-based models (2013)

\section{Post-graduate courses (3.5 ECTS)}

- Complex dynamics in human-environment systems; SENSE (2013)

- Companion modelling; PE\&RC (2014)

Invited review of (unpublished) journal manuscript (1 ECTS)

- Acta Biotheoretica: periodic patters in behaviour of ants and a game theory approach (2013)

Deficiency, refresh, brush-up courses (6 ECTS)

- Simulating emergence; INF group Wageningen University

\section{Competence strengthening / skills courses (3 ECTS)}

- Scientific writing; WGS (2014)

- College geven; WGS (2014)

PE\&RC Annual meetings, seminars and the PE\&RC weekend (1.5 ECTS)

- PE\&RC Weekend first year (2013)

- PE\&RC Weekend last year (2017)

Discussion groups / local seminars / other scientific meetings (4.7 ECTS)

- Systems Biology retreat (2013)

- Workshop sensitivity analysis of complex systems; Wageningen (2013)

- Horti-agent workshop (2014)

- Workshop, social unrest among humans and animals (2014)

- Workshop significant agents (2014)

- Complex adaptive systems seminar, "going potatoes" (2014)

- Modelling and Statistics Network (2014-2017)

- Complex adaptive systems seminar (2015)

- Phd Day IP/OP ccomplex adaptive systems (2015)

- Seminar, fragility of cooperation in a world of change (2015)

- Slotsymposium IP/OP complex adaptive systems 92015) 
- Resilience workshop (2017)

International symposia, workshops and conferences (2 ECTS)

- European Social Simulation Association (ESSA); Warsaw, Poland (2013)

- Models in Population Dynamics and Ecology; Torino, Italy (2014)

- European Social Simulation Association (ESSA); Barcelona, Spain (2014)

- European Social Simulation Association (ESSA); Groningen, the Netherlands (2015)

Lecturing / supervision of practical's / tutorials (6 ECTS)

- Mathematics (2013-2016) 
The research presented in this thesis is part of the Wageningen UR strategic programme 'Complex Adaptive Systems', funded by the strategic funds of Wageningen UR. 
